Florida International University FIU Digital Commons

7-29-1994

\title{
The effect of weathering processes on the vertical turbulent dispersion characteristics of crude oil spilled on the sea
}

\author{
Donald J. Boyé \\ Florida International University
}

DOI: $10.25148 /$ etd.FI14051815

Follow this and additional works at: https://digitalcommons.fiu.edu/etd

Part of the Engineering Commons, and the Environmental Sciences Commons

\section{Recommended Citation}

Boyé, Donald J., "The effect of weathering processes on the vertical turbulent dispersion characteristics of crude oil spilled on the sea" (1994). FIU Electronic Theses and Dissertations. 1777.

https://digitalcommons.fiu.edu/etd/1777 


\section{FLORIDA INTERNATIONAL UNIVERSITY}

Miami, Florida

THE EFFECT OF WEATHERING PROCESSES

ON THE

\section{VERTICAL TURBULENT DISPERSION CHARACTERISTICS OF CRUDE OIL SPILLED ON THE SEA}

A thesis submitted in partial satisfaction of the requirements for the degree of MASTER OF SCIENCE

IN

ENVIRONMENTAL ENGINEERING

by

Donald J. Boyé, Jr. 
To: Dean Gordon R. Hopkins

College of Engineering and Design

This thesis, written by Donald J. Boyé, Jr., and entitled The Effects of Weathering Processes on the Vertical Turbulent Dispersion Characteristics of Crude Oil Spilled on the Sea, having been approved in respect to style and intellectual content, is referred to you for judgement upon its substantial merit.

We have read this thesis and recommend that it be approved

Rudolf Jaffé

Vassilios A. Tsihrintzis

Hector R. Fuentes, Major Professor

Date of examination: 29 July 1994

The thesis of Donald J. Boyé, Jr. is approved

Dean Gordon R. Hopkins

College of Engineering and Design

Dean Richard L. Campbell

Division of Graduate Studies

Florida International University, 1994 
Dedicated to the loving memory of my mother

$\dagger$ 


\section{ACKNOWLEDGEMENTS}

First and foremost, I would like to recognize the special love, support, and understanding of my wife, Pat, and my son, Ryan, which helped me through the difficult times I encountered during the completion of this research effort. Thank you, I am truly blessed and eternally grateful for your love.

I would like to extend my thanks to the committee, Dr. Rudolf Jaffé, Dr. Vassilios A. Tsihrintzis, and Dr. Hector R. Fuentes for their guidance and assistance during this project. I would like to especially recognize Dr. Jaffé for his support and for extending the use of his laboratory facilities for all chemical analyses conducted during this project, without which, the successful completion of this research would not of been possible.

I would like to recognize and gratefully thank the efforts of Rahul Shrotriya for providing the logistical support and vital assistance in the laboratory during this project, without which, the timely completion of this research would have been significantly more challenging.

Thanks to the fine people supporting the Southeast Environmental Research Program (SERP), especially Pete Lorenzo, for providing timely assistance, technical advice, logistical support, and above all, for extending me the utmost courtesy and professionalism.

Special thanks to Walter Conklin at the Mechanical Engineering Lab for providing the guidance, friendly advice, and for facilitating the loan of all the special tools and equipment required to support this project. Thanks are also extended to Dr. Steve Oberbauer for the loan of the Li-Cor pyranometer.

I would like to recognize and gratefully thank the great people supporting Dr. Jaffé in his Laboratory, especially Neghie Hajje, Yumin Gong, Theodore Elisme, for providing the crucial technical assistance and friendly advice, which facilitated the successful completion of the GC/MS characterizations performed during this project.

The timely support of Coulter Corporation with the operation of the DELSA 440 is highly appreciated, and to Mike Whelan for sharing the crucial technical expertise needed during the toxicity evaluations performed on the Microtox Analyzer.

I would like to recognize the fact that this research was funded in part by the Oil Spill Research Center at the University of Miami School of Marine and Atmospheric Sciences, through a grant provided from the United States Coast Guard. Partial funding for equipment and supplies was provided by the FIU Drinking Water Research Center. 


\title{
ABSTRACT OF THE THESIS \\ The Effect of Weathering Processes \\ on the \\ Vertical Turbulent Dispersion Characteristics \\ of Crude Oil Spilled on the Sea
}

\author{
by \\ Donald J. Boyé, Jr. \\ Florida International University, 1994 \\ Miami, Florida \\ Professor Hector R. Fuentes, Major Professor
}

Since the Exxon Valdez accident in 1987, renewed interest has come forth to better understand and predict the fate and transport of crude oil lost to marine environments. The short-term fate of an Arabian Crude oil was simulated in laboratory experiments using artificial seawater. The time-dependent changes in the rheological and chemical properties of the oil under the influence of natural weathering processes were characterized, including dispersion behavior of the oil under simulated ocean turbulence. Methodology included monitoring the changes in the chemical composition of the oil by Gas Chromatography/Mass Spectrometry (GCMS), toxicity evaluations for the oil dispersions by Microtox analysis, and quantification of dispersed soluble aromatics by fluorescence spectrometry.

Results for this oil show a sharp initial increase in viscosity, due to evaporative losses of lower molecular weight hydrocarbons, with the formation of stable water-in-oil emulsions occurring within one week. Toxicity evaluations indicate a decreased EC-50 value (higher toxicity) occurring after the oil has weathered eight hours, with maximum toxicity being observed after weathering seven days. Particle charge distributions, determined by electrophoretic techniques using a Coulter DELSA 440, reveal that an unstable oil dispersion exists within the size range of 1.5 to $2.5 \mathrm{um}$, with recombination processes being observed between sequential laser runs of a single sample. 


\section{TABLE OF CONTENTS}

CHAPTER

PAGE

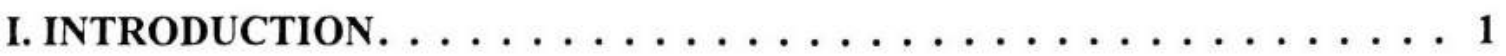

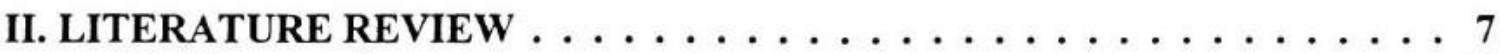

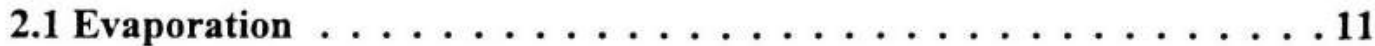

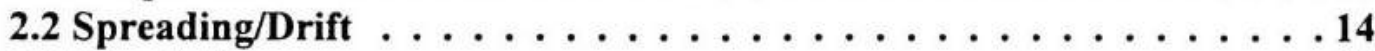

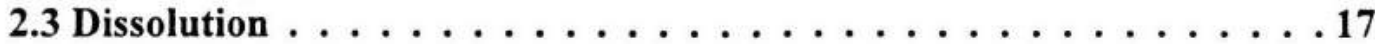

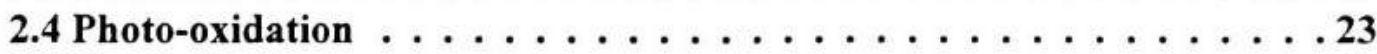

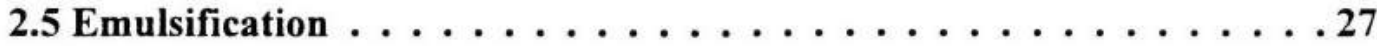

2.6 Sinking/Sedimentation. . . . . . . . . . . . . . . . . . .

2.7 Vertical Turbulent Dispersion . . . . . . . . . . . 37

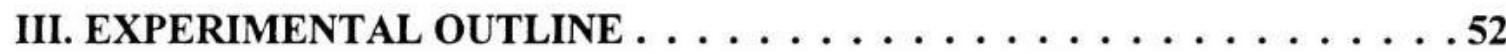

IV. EXPERIMENTAL PROCEDURES . . . . . . . . . . . . . . 56

4.1 Weathering Characteristics . . . . . . . . . . . . . . . 66

4.1.1 Oil Viscosity . . . . . . . . . . . . . 61

4.1.2 Oil-Water Interfacial Surface Tension. . . . . . . . . . .64

4.1.3 Oil Density . . . . . . . . . . . . . . . . . . . . . .

4.1.4 Molecular Characterization by

Gas Chromatography/Mass Spectrometry (GC/MS) . . . . .68

4.2 Vertical Turbulent Dispersion Characteristics . . . . . . . . . 69

4.2.1 Total Oil Concentration. . . . . . . . . . . . 76

4.2.2 Spectrofluorometric Determination of

Total Aromatic Hydrocarbon Concentration . . . . . . .76

4.3 Dispersed Particle Profile . . . . . . . . . . . . . . . . . . . 79

4.4 Relative Toxicity Evaluations. . . . . . . . . . . . . 85

4.5 Experimental Replicability . . . . . . . . . . . . .88

4.6 Quality Control . . . . . . . . . . . . . . . .91 
CHAPTER

PAGE

V. RESULTS AND DISCUSSION $\ldots \ldots \ldots \ldots \ldots \ldots \ldots \ldots \ldots \ldots$

5.1 Physical Properties. . . . . . . . . . . . . . . . . 92

5.1 .1 Oil Viscosity . . . . . . . . . . . . .92

5.1.2 Oil Density/Specific Gravity. . . . . . . . . . . 100

5.1.3 Oil-Water Interfacial Surface Tension . . . . . . . . . 102

5.1.4 Oil Compositional Changes

as Determined by GC/MS. . . . . . . . . . . . . . 104

5.2 Dispersion Characteristics $\ldots \ldots \ldots \ldots \ldots$. . . . . . . 108

5.2.1 Aqueous Phase Oil Concentrations. . . . . . . . . 108

5.2.2 Total Dispersed Hydrocarbons in the Water Column . . . . . . . . . . . . . . . 112

5.2.3 Total Dispersed Aromatic Hydrocarbons in the Water Column . . . . . . . . . . . . . . 119

5.2.4 Toxicity of Dispersed Hydrocarbons, as determined by Microtox . . . . . . . . . . . 124

5.2.5 Particle Charge Distributions and Average Particle Size Observed in the Reactor . . . . . . 126

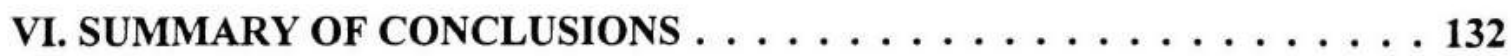

VII. RECOMMENDATIONS. . . . . . . . . . . . . . . . . . 134

VIII. REFERENCES . . . . . . . . . . . . . . . . . . . 137

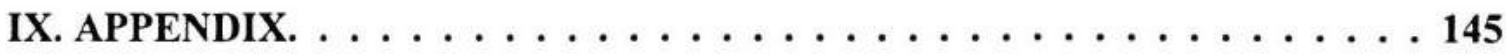




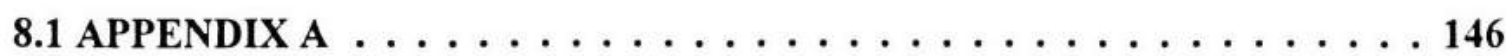

ARABIAN CRUDE OIL (API 28) COMPOSITIONAL DATA TBP AND DISTILLATION DATA

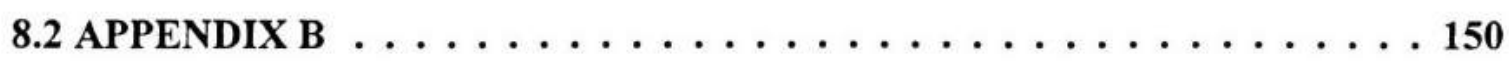

REVIEW OF CONE PLATE GEOMETRY

8.3 APPENDIX C . . . . . . . . . . . . . . 151

CALCULATION OF ENERGY DISSIPATION RATE FOR VERTICAL TURBULENT DISPERSION REACTOR

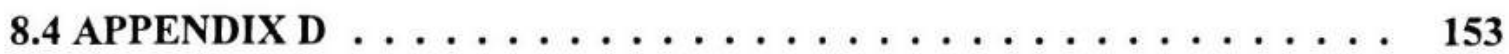

DYNAMIC VISCOSITY OF WEATHERED ARABIAN CRUDE OIL SAMPLES

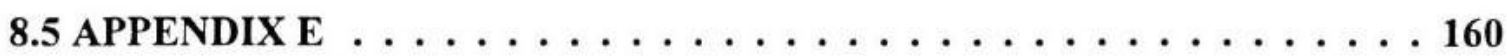

TOTAL SOLAR IRRADIANCE RECORDED DURING WEATHERING PERIOD

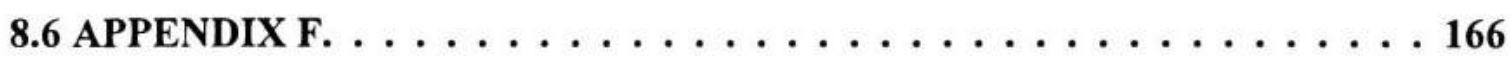

PERCENTAGE OF CRUDE OIL MASS LOST TO EVAPORATION DURING WEATHERING PERIOD

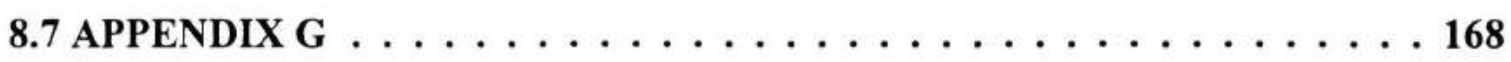

DENSITY AND SPECIFIC GRAVITY OF WEATHERED ARABIAN CRUDE OIL SAMPLES

viii 
LIST OF APPENDICES (continued)

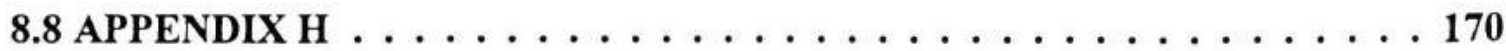

OIL-WATER INTERFACIAL SURFACE TENSION OF WEATHERED ARABIAN CRUDE OIL SAMPLES

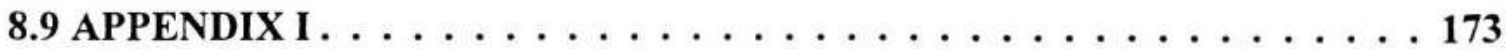

TOTAL ION CHROMATOGRAMS AND SELECTED ION CHROMATOGRAMS FOR WEATHERED ARABIAN CRUDE OIL SAMPLES, AS DETERMINED BY GAS CHROMATOGRAPHY/MASS SPECTROMETRY

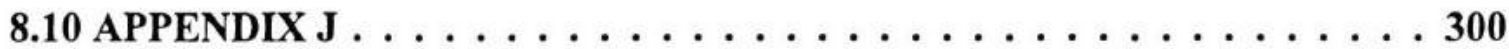

SOLUBLE AROMATIC HYDROCARBONS DETECTED IN WEATHERING FLASK BELOW CRUDE OIL SLICK, AS DETERMINED BY FLUORESCENCE SPECTROMETRY

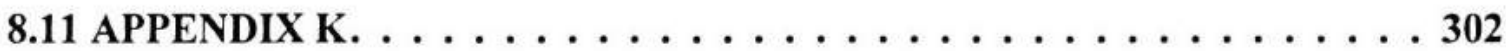

TOTAL OIL CONCENTRATION PROFILE RECORDED WITHIN VERTICAL TURBULENT DISPERSION REACTOR FOR WEATHERED ARABIAN CRUDE OIL SAMPLES UNDER SIMULATED TURBULENCE

8.12 APPENDIX L . . . . . . . . . . . . . . . . . . 309

NORMALIZATION METHODOLOGY FOR TOTAL OIL AND TOTAL AROMATIC HYDROCARBON CONCENTRATIONS RECORDED FROM VERTICAL TURBULENT DISPERSION REACTOR

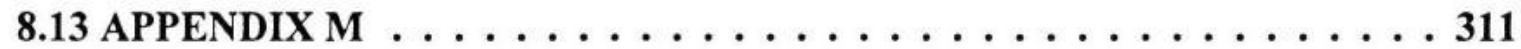

TOTAL AROMATIC HYDROCARBON CONCENTRATION PROFILE, RECORDED WITHIN VERTICAL TURBULENT DISPERSION REACTOR FOR WEATHERED ARABIAN CRUDE OIL SAMPLES UNDER SIMULATED TURBULENCE 
LIST OF APPENDICES (continued)

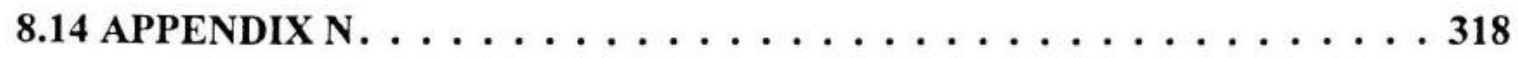

OBSERVED PARTICLE CHARGE DISTRIBUTIONS AND AVERAGE

PARTICLE SIZE DETERMINATIONS; MEASURED BY DELSA 440

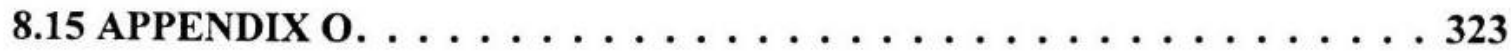

RELATIVE TOXICITY DATA EXPRESSED AS EC-50 VALUES FOR WEATHERED OIL SAMPLES 


\section{LIST OF FIGURES}

FIGURE TITLE

PAGE

Figure 1: Processes Versus Time Elapsed Since The Spill . . . . . . . . . . 9

Figure 2: Crude Oil Weathering Station $\ldots \ldots \ldots \ldots \ldots$

Figure 3 : Brookfield Viscometer Cone Plate Gap Clearance and Correct Sample Volume Verification . . . . . . . . . 63

Figure 4: Distention of the Interfacial Surface Between Two Immiscible Liquids. . . . . . . . . . . . . . 66

Figure 5: Vertical Turbulent Dispersion Reactor . . . . . . . . 71

Figure 6 : Internal 4" Turbulence Generator . . . . . . . . . 72

Figure 7: Adding Crude Oil to the Vertical Turbulent Dispersion Reactor. . . . . . . . . . . . . . . . . 73

Figure 8: DELSA 440 Sample Chamber Assembly. . . . . . . . . 80

Figure $9: \quad$ Location of Stationary Layer $\ldots \ldots \ldots \ldots$

Figure 10: Dynamic Viscosity Versus Weathering Period. . . . . . . . . . 94

Figure 11： Dynamic Viscosity Versus Total Solar Irradiance. . . . . . . . . 95

Figure 12: Dynamic Viscosity Versus Percentage of Crude Oil Mass Lost to Evaporation . . . . . . . . . . . . .96

Figure 13: Percentage of Crude Oil Mass Lost to Evaporation Versus Weathering Period . . . . . . . . . . . .99

Figure 14: Oil Density and Specific Gravity Versus Weathering Period 
FIGURE TITLE

PAGE

Figure 15 : Crude Oil-Water Interfacial Surface Tension Versus Weathering Period . . . . . . . . . . 103

Figure 16: Relative Abundance for Selected Aliphatic Compounds

Versus Weathering Period . . . . . . . . . . . 106

Figure 17: Relative Abundance of Selected Aromatic Compounds

Versus Weathering Period . . . . . . . . . . . . 107

Figure 18 : Gas Chromatogram for Hopane Series ( Ion 191 ) . . . . . . . 109

Figure 19: Soluble Aromatic Hydrocarbons in Weathering Flask

Versus Weathering Period . . . . . . . . . . 110

Figure 20: Total Dispersed Hydrocarbons in Water Column

Versus Weathering Period

[ Turbulence Duration - 5 minutes ] . . . . . . . . 114

Figure 21: Total Dispersed Hydrocarbons in Water Column

Versus Weathering Period

[ Turbulence Duration -180 minutes $] \ldots \ldots \ldots \ldots$

Figure 22: Total Dispersed Hydrocarbons in Water Column

Versus Weathering Period

[ 3 Hours After Turbulence Generator Shutdown ] . . . . . . 118

Figure 23 : Total Dispersed Aromatic Hydrocarbons in Water Column

Versus Weathering Period

[ Turbulence Duration - 5 minutes ] . . . . . . . . 120

Figure 24: Total Dispersed Aromatic Hydrocarbons in Water Column

Versus Weathering Period

[ Turbulence Duration - 180 minutes ] . . . . . . . . . 121 


\section{LIST OF FIGURES ( continued)}

FIGURE TITLE

PAGE

Figure 25: Total Dispersed Aromatic Hydrocarbons in Water Column

Versus Weathering Period

[ 3 Hours After Turbulence Generator Shutdown ] . . . . . . . 122

Figure 26: Microtox EC-50 Values at Bottom of Reactor

Versus Weathering Period . . . . . . . . . . 125

Figure 27 : Particle Recombination Recorded by Coulter DELSA 440 . . . 130

Figure 28： Post Experimental Calibration Check of Coulter DELSA 440 $\ldots 131$ 


\section{LIST OF TABLES}

TABLE NUMBER

1

2
TITLE

PAGE

Comparison of Replicate 4-day Weathering Periods ................. . . . . . .

Observed Zeta Potentials and Average Particle Sizes. . . . . . . . . . . . . . . . . . . . 129 


\section{INTRODUCTION}

It is estimated that between 10 and 100 million tons of petroleum products are lost to the world's oceans annually (Sutton \& Calder, 1974). These inputs stem from a wide variety of sources, which include among others, natural sources, in the form of marine sub-surface seeps and sediment erosion, municipal and industrial waste discharges, urban runoff, and accidental discharges related to the handling and transportation of crude oil and petroleum distillates. However, the marine environment has an enormous capability to assimilate and degrade these petroleum hydrocarbon compounds through effective physical, chemical, and biological weathering processes, which are initiated immediately upon release. The oftentimes competitive processes which determine the final disposition of the oil include surface spreading, drifting with prevailing winds and currents, evaporation, photo-oxidation, dissolution; dispersion of discrete oil particles into the water column under the influence of surface turbulence, spray injection into the air during severe weather, water-in-oil emulsification, adsorption onto suspended particulate matter, sinking, sedimentation, biological uptake, and microbial degradation.

For the most part these hydrocarbon discharges are unobtrusively lost to the marine environment leaving no readily apparent environmental impacts; this is evident by the fact, that the world's oceans and coastlines do not reflect the serious degradation that would be expected from the large volume of petroleum products discharged to date. However, in the case of tanker accidents at sea, which generally account for one-half the combined discharge 
from both municipal and industrial sources annually (Borthwick \& Joynes, 1992), an entirely different scenario develops with oftentimes catastrophic environmental consequences. When large quantities of crude oil are released in coastal waters, there is insufficient time for weathering processes to adequately degrade the oil before reaching the shore, resulting in serious and long-term environmental impacts. These may include lethal effects to many sea birds and mammals from direct smothering, and lowering of primary productivity of the affected area. Other sub-lethal effects can also include disruption of normal feeding patterns, sex attraction, host recognition in symbiotic relationships and other intricate social interactions, and growth suppression in some organisms (Winters et al., 1976; Boylan \& Tripp, 1971). These accidental discharges of petroleum products to the marine environment are unfortunate hazards that modern society must be prepared to accept when continuously increasing quantities of crude oil products are transported over great distances, to provide the energy needs of industrialized and developing nations.

Poignant examples exist of the severe environmental consequences of large releases of petroleum oil in sensitive coastal areas, often a result of human error in vessel handling. Among the most destructive in recent times are the super tanker $M / V$ Braer running aground and breaking up off the Shetland Islands in 1993 losing the entire cargo load of light distillate oil; and, in 1987 the supertanker Exxon Valdez running aground in Prince William Sound spilling 242,000 barrels of crude oil, becoming the worst oil spill disaster in U.S. history. 
The worst tanker accident ever on record occurred in 1978 when the supertanker Amoco Cadiz ran aground and broke up off the coast of Brittany losing 223,000 tons of crude oil and contaminating $320 \mathrm{~km}$ of shoreline.

While these tragic maritime accidents may have gained sensational coverage in the media, there are a number of additional, albeit less severe, tanker incidents which occur on a seemingly regular basis. Data compiled worldwide for the period of 1974 to 1980 indicate that 57 tanker spills of crude oil in excess of 10,000 barrels had occurred, with an additional 57 spills in the range of 1,000 to 10,000 barrels (Amstutz, 1980). Additionally, Borthwick \& Joynes (1992) reported that during the year 1990, the U.K. Department of Energy recorded 791 tanker spills off the coast of the United Kingdom alone.

Since the occurrence of the Exxon Valdez accident in 1987, there has been a resurgence of interest by the U.S. Federal Government to push, not only for stricter regulations on the way petroleum products are transported, (i.e., requiring double hulls on newly constructed tankers), but also on furthering our understanding on the fate and transport of petroleum hydrocarbons once released in the marine environment. A more accurate prediction of the time dependent changes in the physical and chemical properties of the oil, along with the spreading characteristics of the slick for various initial conditions (e.g., wind speed, sea-state, temperature, and initial oil properties), provides crucial information for improving the forecasting of slick trajectories to define the possible extent of shoreline contamination. In 
addition. it allows for the more effective selection of counter-measure technology. which if properly deployed in the right place and with the proper level of response, would mitigate or eliminate environmental impacts associated with large near-shore oil spills. Finally, it provides new insights for predicting the vertical dispersion characteristics of the crude oil, which is essential in estimating the volume of oil lost beneath the surface. The vertical turbulent dispersion of oil particles and the dissolution of soluble fractions into the water column is an important consideration in terms of the overall environmental impact of an oil spill on the marine community. The combination of these processes may be in fact the largest contributor to the overall disappearance of the oil spill in the presence of turbulent sea conditions (Lynch, 1987). These components of the combined weathering process are critical, for once the oil is lost beneath the surface of the overlying slick, it is difficult if not impossible to recover by any means.

The formation of small oil droplet dispersions in the presence of sufficient ocean surface turbulence and duration can be carried to great depths, due to the combination of two factors: significant sub-surface eddy currents in the wake of breaking waves, and the extremely small rise velocities associated with these size particles. Forrester (1971), surveying the distribution of oil particles within the water column at the site where the tanker Arrow ran aground in 1970, was able to positively detect oil particles as deep as $80 \mathrm{~m}$; due to the advection of oil particles by sub-surface currents, he was able to detect traces of oil as far as $250 \mathrm{~km}$ from the spill source. Similar findings were also observed by scientists studying the 
Amoco Cadiz spill. In that incident, near-bottom waters were found to generally contain more total oil than water samples taken at a 2-meter depth, even with oil still floating at the surface. Actually, the highest concentration of total oil (140 ppb) detected offshore was observed at a depth of $65 \mathrm{~m}$ and at a distance of $16 \mathrm{~km}$ northeast of the site where the Amoco Cadiz went aground (Wolfe, 1978). Considering that a dispersion containing $1 \mathrm{ppm}$ of oil distributed within a one square kilometer section of water column to a depth of 1 meter will contain oil in excess of 1 ton (Freegarde et al.,1971), these findings highlight the importance of understanding vertical dispersion dynamics. It is an extremely important issue to address in terms of better understanding and quantifying spreading dynamics, and in assessing the adverse ecological impacts that an oil spill may inflict on aquatic communities, not only at the spill site, but in areas quite distant from the accident.

A review of the modeling of oil spill trajectory and fate dependent processes is provided by Al-Rabeh et al. (1989); Huang (1983); Spaulding (1988); and Reed et al. (1989); it is a complex numerical simulation with only finite capabilities on providing information which comprehensively predicts the long-term fate of oil spilled at sea. The dependent variables driving the fate simulation of oil are so numerous that modeling would require nearly an individualistic effort (i.e., on a spill by spill basis) to completely predict fate processes with any high degree of accuracy. The fate of crude oil spilled on the sea is dependent on: (1) existing local meteorological conditions, such as prevailing wind speed and direction, air temperature, and intensity of solar radiation (dependent on percent cloud cover and localized 
ozone layer thickness); (2) existing sea conditions, such as significant wave height. probability of breaking wave events within the slick area, water temperature, water depth, salinity, concentration of suspended particulate matter (SPM), and the presence of dissolved organic matter (DOM); (3) geographical conditions, including fetch distances and type of coastline, (i.e., high energy rocky shores), where dispersed oil droplet formation and the subsequent sedimentation by SPM interactions are the major concern compared to low energy estuarine backwaters, where shallow quiescent waters may promote the accumulation of toxic dissolution products, (Payne \& McNabb, Jr., 1985; Owens, 1978); and most importantly, (4) the physical properties of the oil, such as chemical composition, viscosity, density, oil-water interfacial tension, slick thickness, and slick area.

According to Spaulding (1988), calibrated algorithms for photo-oxidative processes, dispersed oil-SPM interactions, and long-term biodegradation are still required, along with a more comprehensive characterization of the vertical turbulent dispersion of oil droplets into the water column. Therefore, the effort presented within this study is to characterize the effects that weathering processes have on the physical properties and chemical composition of an Arabian Crude oil (API 28), and to correlate these time defendent changes to the susceptibility of the oil to vertical turbulent dispersion under simulated surface turbulence. The oil dispersed beneath the slick under fixed turbulent energy inputs was analyzed for total oil and total aromatic hydrocarbon concentration. The toxic effects of these dispersions was then quantified by bacterial bioluminescence assay experiments using a Microtox analyzer. 
Research conducted in support of this study was designed to yield important insights for those concerned with fate and effects modeling, because it provides a link between the time when the oil spill occurs and the time when the oil slick encounters sufficient turbulence to induce vertical dispersion (defined herein as the weathering period), to a characterization of the relative magnitude of oil which is vertically dispersed within the water column. The quantification of the relative toxicity of these dispersions is important when assessing their time variant potential to impose adverse environmental impacts.

\section{LITERATURE REVIEW}

The fate and final disposition of crude oil spilled on the sea is strongly linked to vertical dispersion dynamics acting upon the slick. The magnitude of this weathering mechanism is dependent and assisted by the contributions of a number of interactive and oftentimes competitive physical and chemical processes, which include spreading, drifting with prevailing winds and local current patterns, evaporation of lighter weight hydrocarbon compounds, dissolution of the soluble fractions and the formation of additional highly soluble hydrocarbon intermediates from photo-oxidative degradation, emulsification and formation of tar balls, sinking, sedimentation under the influences of ambient suspended particulate matter, and ingestion of oil by zooplankton with its subsequent incorporation into fecal pellets. To fully understand the vertical turbulent dispersion characteristics of crude oil spilled at sea, each of these weathering processes have been isolated in the literature herein, in order to accurately define the contribution of each process to the overall 
disappearance of the surface slick. and the resultant effect of each process on the vertical turbulent dispersion process. The relative magnitude and time span of the weathering processes acting upon oil spilled on the sea are depicted in Figure 1

As an introductory overview, the vertical turbulent dispersion of oil is defined as the incorporation of oil droplets into the water column under the influences of surface turbulence. Once the oil mass is entrained in the water column, it is subsequently exposed to sub-surface advection by prevailing currents, which expands the area effected by the spill. The oil dispersions are also exposed to sedimentation, depending on oil droplet size, due to adsorptive interactions with suspended particulates, which increases the environmental impacts to benthic communities. The formation of these oil droplets initiates with the shearing of an oil mass from the underside of the coherent slick under the influences of a number of forces. The primary causal factor associated with this phenomenon is generally considered to be the large energy dissipation rates associated with breaking wave conditions, but vertical dispersion can also occur in the presence of rain or sufficient upper level water circulations patterns formed in the presence of prevailing winds.

The incorporation of large parcels of oil into the water column under breaking waves results in the formation of numerous discrete oil particles which may range in size from a few microns to a few millimeters (Mackay et al., 1983). 


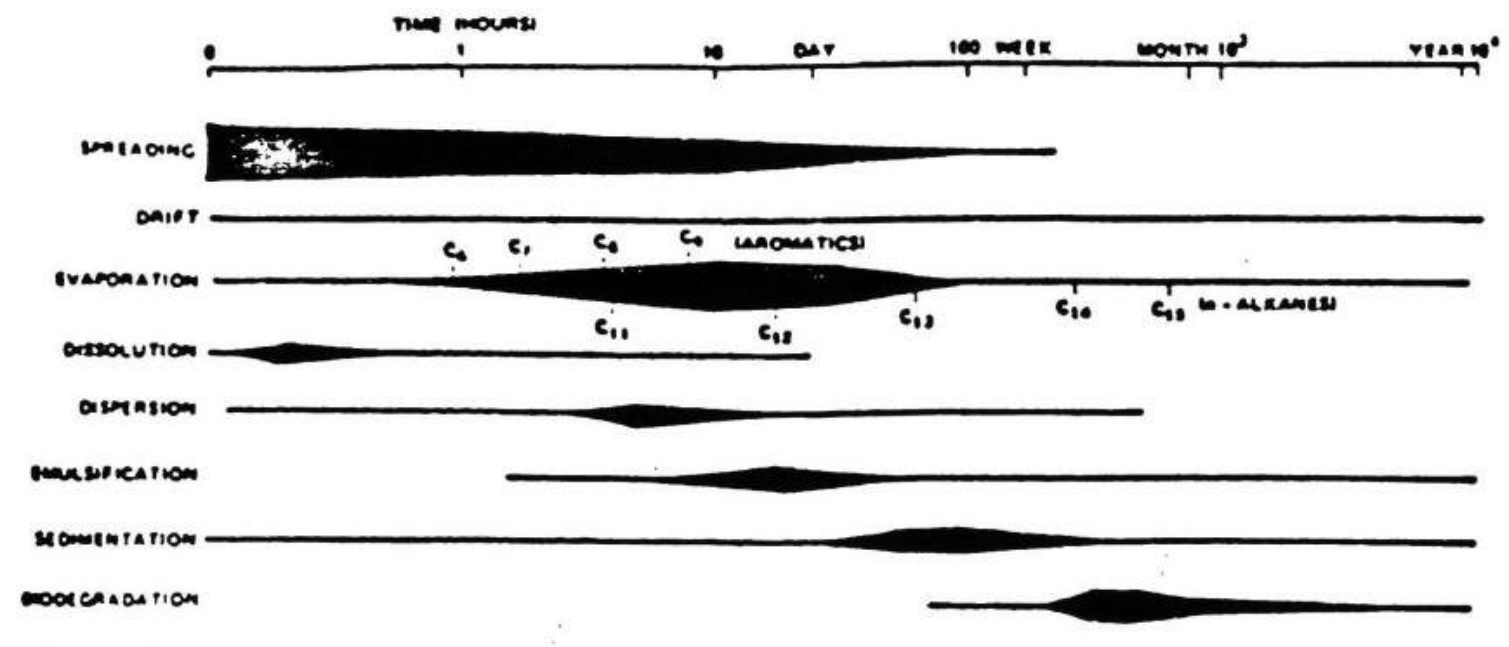

The line length indicstes the probuble timesaen of anv pro. cese the line width mdicates the relative magnitude of the process through time and in relevion to other conumoorar processer.

\section{Figure 1}

Processes versus Time Elapsed Since The Spill

(Wheeler, 1978) 
The particles found at the upper end of this particle size distribution will resurface and recoalesce with the surface slick at a rate proportional to the second-power of the droplet size and to oil-water density differences according to Stokes Law.

$$
W=g_{o}^{2}\left(\rho-\rho_{w}\right) /\left(18 \rho_{w} v_{w}\right)
$$

where:

$$
\begin{array}{ll}
\mathrm{W} & =\text { resurfacing rate }(\mathrm{m} / \mathrm{sec}) \\
\rho & =\text { density of the oil }\left(\mathrm{kg} / \mathrm{m}^{3}\right) \\
\rho_{\mathrm{w}} & =\text { density of water }\left(\mathrm{kg} / \mathrm{m}^{3}\right) \\
v_{\mathrm{w}} & =\text { kinematic viscosity of water }(\mathrm{cSt}) \\
d_{o} & =\text { diameter of the oil droplet }(\mathrm{m})
\end{array}
$$

Equation [1] says that the smaller droplets have larger residence times under the slick due to negligible buoyant forces. These droplets generally resurface as a sheen of oil trailing behind the main body of the surface slick. If these small droplets are propelled deep enough, they are generally considered permanently incorporated into the water column (Al-Rabeh et al., 1989; and Mackay et al., 1983), and are then subjected to sub-surface advection by deep ocean currents which may transport the oil long distances away from the original spill site and in directions counter to the prevailing direction predicted by traditional surface slick trajectory models. 
The rate at which oil is incorporated into the water column is a function of a number of parameters including the prevailing sea state (i.e., wave height, wave period. frequency of breaking wave events within the slick area), and the physical and chemical characteristics of the slick during the period the oil is encountering sufficient turbulence (i.e., viscosity, density, oil-water interfacial tension, and slick thickness) ( Al-Rabeh et al., 1989). The point at which oil becomes susceptible to vertical dispersion and the subsequent rate at which oil is incorporated into the water column is an important consideration, for once the oil is lost beneath the surface of the slick, it is virtually impossible to recover by any available countermeasure technology, and therefore, must be quantified to determine environmental impacts of an oil spill on benthic communities.

\section{$\underline{2.1 \text { Evaporation }}$}

The evaporation of lighter weight hydrocarbons, predominantly n-alkanes, is initiated shortly after the release of oil (Figure 1), and is considered to be the most significant factor in the overall disappearance of the slick; dependent on oil type, especially during the first 48 hours (Aravamudan \& Marsh, 1981; Payne \& McNabb, Jr., 1985; Davis \& Gibbs, 1975; Buchanan and Hurford, 1988; and Frankenfeld, 1973). Evaporation rates generally far exceed the rates of all other processes, and may exceed the dissolution rate of aromatic hydrocarbons by a factor of 100 (Payne \& McNabb, Jr., 1985); for aliphatic hydrocarbons the rate of evaporation may be 10,000 times greater than the dissolution rate (Harrison et al., 1975; and McAuliffe, 1976a). This factor is crucial from an environmental standpoint because the 
more toxic water soluble compounds found in the surface slick, such as benzene and toluene. are quickly removed from the spill when allowed to equilibrate with the air (Frankenfeld. 1981; and Page et al., 1983).

The percentage of the original mass of the slick lost to evaporation, as stated in the literature, can vary significantly, from $20 \%$ to $40 \%$ (Spaulding, 1988; Lynch, 1987; Raj and Griffiths. 1979; and Venkatesh, 1988), under most situations, to as high as $60 \%$ in extreme environmental conditions, of solar intensity and temperature, found in the Arabian Gulf region (Al-Rabeh, 1989). The evaporation rate from a slick is influenced by the chemical characteristics of the original crude, the vapor pressure of the oil as affected by the percent of lighter hydrocarbon fractions lost to the atmosphere (Spaulding, 1988; Mackay et al, 1983; and McAuliffe, 1976a), sea-state, meteorological conditions (i.e., wind speed, air and water temperatures, and local solar radiation levels which are dependent on the percentage of cloud cover and the ozone layer thickness above the slick) (Lee \& Downum, 1991), and the relationship that decreasing slick thicknesses are associated with increasing surface areas, which increase the mass transfer rates of the volatile fraction (Spaulding, 1988; Berridge et al., 1968; and Huang, 1983).

The evaporation of lighter hydrocarbons alters the chemical composition and physical characteristics of the oil. The loss of the volatile fraction causes a significant increase in the viscosity of the oil, and increases the relative concentrations of sulfur, metals (i.e., nickel and 
vanadium), waxes, and asphaltenes. The effect of these alterations are self-limiting on the rate of evaporation, due primarily to the increase in viscosity and the tendency of the oil towards emulsification which reduces the diffusion of volatile fractions to the air-oil boundary layer (McAuliffe, 1976a).

The effect of evaporation on the vertical turbulent dispersion characteristics of crude oil is to increase the dispersion potential, due to an increase in the density of the oil as the lighter hydrocarbons are preferentially lost. Sivadier \& Mikolaj (1981) and Harrison et al.,(1975) noted that under sea-state four conditions virtually all of the volatile hydrocarbons were lost in the first two hours, with essentially all the lighter hydrocarbons boiling up to $216^{\circ} \mathrm{C}$ (C12) lost in the first 24 hours. The loss of lighter weight hydrocarbons, combined with the incorporation of water during emulsification, increases the specific gravity of the remaining oil. For example, the residuum contained in the boiling fraction above $1000^{\circ} \mathrm{F}$ for a Kuwait crude, which forms $27 \%$ of the total mass of the fresh crude, was found to have a specific gravity of 1.023 (Berridge et al., 1968). The specific gravity of seawater is approximately 1.025 which makes this residue highly susceptible to sinking, especially in the presence of suspended particulate matter (SPM). The result of evaporation and the associated effects of increasing densities, makes the oil more susceptible to sinking, and therefore, subject to long range transport by deep ocean currents (Sivadier \& Mikolaj, 1981; and Berridge et al., 1968). 


\section{$\underline{2.2 \text { Spreading/Drift }}$}

The spreading of crude oil spilled on the sea is a physical process which is controlled by the driving forces of gravity and surface-tension, and retarded by the combined forces of viscosity and inertia. The spreading of crude oil (Figure 1) is the first process contributing to weathering which acts upon the slick; beginning immediately upon the release of oil to the sea surface, and continuing until the slick is reduced to a sub-millimeter thickness, surrounded by sheens considered to be mono-molecular (Mackay et al., 1983). The process of oil spreading increases the surface area to volume ratio of the spill, which enhances the rate of evaporation and dissolution (Payne \& McNabb, Jr., 1985), and enlarges the area influenced by photo-oxidative degradation. The results of these weathering mechanisms, combined with the resultant reduction of slick thickness during the spreading process, all put the oil more at risk to water-in-oil emulsification and vertical dispersion mechanisms, which expands the area affected by the related environmental impacts of the spill (Elliott $e t$ al., 1986). In addition, the spreading of oil on the sea surface increases the range area of containment and the magnitude of cleanup efforts (Huang, 1983).

The spreading of oil is generally considered to occur in three distinct phases (Al-Rabeh et al., 1989; and Lehr et al., 1984). The first phase is defined as gravity-inertial (i.e., driven by gravity and impeded by the inertia of the coherent oil mass) and dominates only during the first few minutes after a spill, except in extremely large oil discharges. Phase II, defined as gravity-viscous, is the primary mechanism starting approximately 1 hour after the spill and 
lasting roughly 7 days. This phase of the slick spreading is considered to be the most useful phase for estimating oil spill sizes in any type of fate modeling. The spreading rate during this phase has been determined to be a function of the initial oil volume lost, gravity effects, elapsed time after the spill, kinematic viscosity and density of the oil, density of water as related to salinity (Lehr et al., 1984), and additionally by the chemical composition of the oil, pour point, and by wind speed and current.

Lehr et al. (1984) predicted that the spreading of oil resembles an ellipse, with the major axis aligned with the prevailing wind vector by the following relationships:

$$
\begin{array}{ll}
\mathrm{A} & =(\pi / 4) \mathrm{QR} \\
\mathrm{Q} & =1.7\left[\left(\rho_{\mathrm{w}}-\rho_{\mathrm{o}}\right) / \rho_{\mathrm{o}}\right]^{1 / 3} \mathrm{~V}^{1 / 3} \mathrm{t}^{1 / 4} \\
\mathrm{R} & =\mathrm{Q}+0.03 \mathrm{~W}^{4 / 3} \mathrm{t}^{3 / 4}
\end{array}
$$

where:

$\mathrm{Q} \quad=$ length of the minor axis

$\mathrm{R} \quad=$ length of the major axis

$\rho_{w} \quad=$ water density

$\rho_{\mathrm{o}} \quad=$ oil density

$\mathrm{V} \quad=$ the initial volume of the spill in barrels

$\mathrm{W} \quad=$ the wind speed in knots

t $\quad=$ the elapsed time after the spill 
Additional concerns during Phase II, are the effects of vertical dispersion on the spreading dynamics of the spill. The forces of surface turbulence will result in a size spectrum of oil particles in the water column. The larger particles being more buoyant will remain closer to the surface with smaller resultant residence times. Therefore these particles will be advected at the speed of the surface drift in the direction of the wind and waves. In contrast. smaller droplets will be less buoyant and will be mixed down by the turbulence to depths of several meters where the advective flow will be weaker. The smaller droplets will have significantly larger residence times in the water column and will trail behind the main body of the slick as submerged particles, eventually resurfacing, forming a tail of oil sheened across the water surface (Elliott et al., 1986).

Phase III, defined as surface tension-viscous, is the sole mechanism acting upon the slick after the first week. The spreading characteristics at this point are driven by the surface active constituents having functional groups containing oxygen, nitrogen, and/or sulfur which act as natural dispersants. The adsorption of these surface active species at the oil-water interface aids in the reduction of the interfacial energy of the boundary, which assist in the emulsification process, and reduces the energy required by wind and waves to further disperse oil into the water column as discrete oil particles (NAS, 1973).

The drifting of oil slicks, influenced primarily by the forcing functions of wind and currents, (Beer et al., 1983), has the adverse effect of bringing oil spilled offshore into coastal areas 
where energy dissipation rates are significantly greater, promoting emulsification mechanisms and dispersion of oil droplets into the water column. The energy dissipation rate of the surface layer in the coastal regime is significantly higher due to the larger presence of breaking wave conditions. The vertical turbulent dispersion of oil into the water column has been found to be linearly related to this energy dissipation rate (Delvigne \& Sweeney, 1987).

The speed of the drifting oil is generally considered to be approximately 3 percent of the wind velocity (Johansson et al., 1980; Huang, 1983; McAuliffe, 1976a; Payne \& McNabb, Jr., 1985; and Spaulding, 1988), superimposed with 100 percent of the current velocity (Lynch, 1987). However, field data collected by Lange \& Huhnerfuss, (1978) has shown that $3 \%$ drift velocities may be understated; in the presence of deep water gravity waves, the drift velocity may be actually $25-30 \%$ higher due to the additional mass transport and increased vorticity exhibited in the presence of larger waves. The drifting of oil at sea, in the absence of interactions with coastal land masses and near-shore currents, has been also found to shift 20-30 degree to the right, in northern latitudes, because of the Coriolis Effect.

\section{$\underline{\text { 2.3 Dissolution }}$}

The dissolution of hydrocarbons from under a surface slick is generally not considered to play a significant role in the overall disappearance of crude oil spilled at sea. In terms of defining the resultant environmental impacts to marine ecosystems, understanding the rate of hydrocarbon dissolution and their association with adverse toxicological effects may be 
of prime importance (Payne \& McNabb. Jr., 1985; Huang, 1983: and Mackay \& Shiu. 1976). As shown in Figure 1, the dissolution process is short-lived, acts primarily during the first few hours after a spill, and faces direct competition from the evaporative process. As stated previously, the highly soluble lighter hydrocarbon fraction is also extremely volatile, and will evaporate quickly. The dissolution of these hydrocarbon compounds can be facilitated by the vertical dispersion of oil droplets into the water column; with increased surface areas for mass transfer, larger dissolution rates can be expected.

The dissolution of hydrocarbons process is a relatively minor process compared to the evaporation rate. Experiments conducted to evaluate the relative rate of these competitive processes have allowed pairs of compounds with similar vapor pressures, but drastically different solubilities, to equilibrate with water, i.e., benzene and cyclohexane (McAuliffe, 1976a) or cumene and nonane (Harrison et al., 1975). Concentrations of the two compounds were measured over time in the aqueous phase and for the remaining slick, and no appreciable difference in the aqueous phase concentration was noted. The rate of dissolution estimated to be at roughly $1 \%$ of the evaporation rate. The competitive rate dynamics for these low molecular weight hydrocarbons will partially determine if residence times are sufficiently long for these compounds to be transported to adjacent shorelines (McAuliffe, 1976b). 
The compounds most susceptible to dissolution (i.e., benzene and toluene) are also rapidly depleted from the slick by evaporation. The peak concentration of dissolved hydrocarbons occurs approximately 8 to 12 hours after the initial release of the oil. The concentration of dissolved hydrocarbons is then observed to exponentially decay because of the turnover of water volume under the slick due to advection, and the evaporative loss of these volatile hydrocarbons when they return to the surface and are exposed to the air-oil mass transfer boundary (Payne \& McNabb, Jr., 1985; Harrison et al., 1975; and Payne et al., 1983). The most profound effect of dissolution would occur in quiescent water under the cover of thick oil slicks, where toxic concentrations of aromatic compounds could be realized and maintained for extended periods of time (Owens, 1978). The water soluble aromatic and aliphatic hydrocarbons extend sub-lethal effects to marine organisms at concentrations of 10$100 \mathrm{ppb}$, lethal toxicity at $0.1-1.0 \mathrm{ppm}$ for most larval stages, and lethal effects at $1-100 \mathrm{ppm}$ for most adult organisms (Harrison et al., 1975). A comprehensive review of the effects of oil on marine mammals can be found in Geraci \& St. Aubin (1988).

The concentration of benzene and toluene, which constitute only $2 \%$ of the total mass of crude, will make up $50 \%$ of the total dissolved hydrocarbons in the water column (McAuliffe, 1973). The more persistent aromatic hydrocarbons in the water column are indanes, naphthalenes, acenaphthenes, acenapthalenes, flourenes, and phenanthrenes (Frankenfeld, 1973). Additionally, the intermediate products of photo-oxidative reactions during weathering would increase the concentrations of two-ring oxygenated compounds 
(i.e., naphthol) (Frankenfeld, 1981). Siron et al., (1993) observed that benzene. naphthalene. and their branched homologs accounted for the largest proportion of the total dissolved oil compounds, and were largely responsible for inhibiting bacterial growth in toxicity evaluations. The concentrations of these compounds, considered to be the most toxic, were observed to increase in the aqueous phase during laboratory experiments conducted by Boylan \& Tripp (1971) in the presence of increased surface turbulence. This has important implications from an environmental impact perspective. In the presence of sufficient turbulence at the time the oil is spilled and relatively unweathered, significant quantities of potentially toxic hydrocarbons, including the most lethal low molecular weight hydrocarbons like benzene and toluene could be carried quite deep into the water column precluding the loss of these components to evaporation.

The large quantity of small oil droplets carried deep into the water column by breaking waves and turbulent eddies would significantly increase the boundary layer across which the mass transfer of soluble hydrocarbons could occur. For example Mackay et al. (1981) concluded that a $1-\mathrm{mm}$ thick oil slick has an area to volume ratio of $1000 \mathrm{~m}^{2} / \mathrm{m}^{3}$, and a 10 -um oil droplet would have a ratio of $3.01 \times 10^{5} \mathrm{~m}^{2} / \mathrm{m}^{3}$. Drops of such small diameters would reach equilibrium with the surrounding water very rapidly and would lose much of their soluble material. 
The dissolution rate of hydrocarbons is related primarily to component solubility and the individual mole fraction of each component in the slick; additional factors include diffusivity coefficients, $\mathrm{pH}$, mass transfer surface area, oil temperature, volume of discharged oil, concentration of other hydrocarbon species in solution, concentration of dissolved organic and colloidal materials, and sea-state conditions at the time of the spill (Huang, 1983).

The solubilization of hydrocarbons in water is directly related to the size of the hydrocarbon molecule, with larger molecules being less soluble. Typical solubility values are generally in the range of $1-10 \mathrm{ppm}$ for aromatics and $0.0001-0.01 \mathrm{ppm}$ for $\mathrm{n}$-paraffins. This phenomenon was analyzed by Bohon \& Claussen (1951), who proposed that the volume occupied by the hydrocarbon in a water solution is related to the solubility of the molecule. Hermann (1972) stated that a more idealized quantity that is readily computed is the area of the cavity surface which connects the centers of all the water molecules in the first layer around the solute. The area defined in this manner was found to be linearly related to the logarithm of the hydrocarbon solubility. The salinity of the water column is also related to the hydrocarbon solubility. The solubility in seawater being approximately $70-80 \%$ of that for distilled water (Delvigne et al., 1986; and Mackay et al., 1983). Higher salinities reduce the solubility of hydrocarbons due to the salting out effect, which could have important implications if pollutants are transported through salinity gradients (Sutton \& Calder, 1974). Dissolved salts in water decrease the solution volume, which in turn makes cavity formation energetically unfavorable (Shaw, 1976). Detailed information on solubilities for 
hydrocarbons can be found in McAuliffe (1966) and Mackay et al. (1980); a comprehensive list of the solubility of hydrocarbons in salt water can be found in Yaws (1992).

Shaw (1976) also determined that a threshold concentration exists for soluble hydrocarbons below which the average spacing of hydrocarbon molecules is sufficiently large that their ordered region exist independently of each other. At higher concentrations there appears to be an interaction between the ordered regions of water molecules surrounding the hydrocarbon molecule, causing one solute to affect the solubility of another. For example, the aqueous solubility of phenanthrene, in the presence of saturation concentrations of biphenyl and naphthalene, was observed to fall from $1.07 \mathrm{ppm}$ to $0.92 \mathrm{ppm}$.

The concentration of dissolved organic matter in the water column has also been found to effect the solubilization of certain hydrocarbons, which has important implications for dispersion and sedimentation of hydrophobic pollutants in estuarine and coastal areas (Boehm \& Quinn, 1973). In natural waters, dissolved organics, generated from sediments and sewage outfalls (Delvigne et al., 1986), may serve as surface active agents to solubilize oil. The reduction of dissolved organic matter in water samples analyzed from Narragansett Bay, while having no effect on aromatic hydrocarbon solubilities, resulted directly in a $50-99 \%$ reduction in the amount of n-alkanes and isoprenoid hydrocarbons solubilized (Boehm \& Quinn, 1973). This relationship was suggested to be linked to the incorporation of hydrocarbons into micelle formed by surface active humic materials. A reduction in the 
concentration of solubilized material in the aqueous phase reduces their potential for bioaccumulation because of their reduced bioavailability (Jaffé, 1991). The n-alkane solubility was also affected by the $\mathrm{pH}$ of water solutions in the presence of humic substances, and reached a maximum value with respect to ionic strength at 0.3 .

\subsection{Photo-Oxidation}

Photo-oxidative reactions, initiated by sunlight, alter the originai properties of the crude oil, which results in the formation of oxidized compounds containing polar functional groups, such as peroxides, aldehydes, ketones, carbonyl compounds, alcohols, phenols, and fatty acids (Payne \& Phillips, 1985b; Hansen, 1975; Berridge et al., 1968, and Larson et al., 1977) These compounds have increased solubilities (Burwood \& Speers, 1974; Siron et al., 1993; and Spaulding, 1988), and are generally more toxic than their parent compounds, which is an important aspect in assessing the environmental impacts of oil spilled at sea (Andersson \& Bobinger, 1992; Blackman \& Law, 1980a; Ehrhardt \& Douabul, 1989; Larson et al., 1979; and Gala \& Giesy, 1992). For example, the solubility of naphthalene is $35 \mathrm{mg} / \mathrm{L}$, whereas the solubility of its oxygenated counterpart, 2-naphthol, is $1000 \mathrm{mg} / \mathrm{L}$ (Larson et al., 1979).

The build up of oxygen containing polar structures leads to a decrease in the oil-water interfacial tension which reduces the energy required to force oil into the water column under the action of surface turbulence which enhances the dispersion of oil droplets (Hansen, 1975). Burns (1993) provided evidence for the importance of including hydrocarbon 
oxidation products in environmental assessment studies through the analysis of 10 samples of bivalve tissue collected as part of the impact assessment of the Exxon Valdez spill. Results of the analysis by ultraviolet spectroscopy revealed that the ongoing effects of photooxidation and biodegradation processes created a complex assembledge of toxic intermediate oxidation products which are bioaccumulated in marine organisms. In another study, the toxicity of a $\# 2$ fuel oil was observed to reach a maximum during laboratory experiments in approximately 24 hours, because of the formation of reactive peroxides. The toxicity, turbidity, acidity, or the presence of photo-oxidative by-products was unaltered in control experiments kept in the dark, but a noticeable toxic effect on natural bacteria was observed after a few hours of illumination (Larson et al., 1977; Pengerud et al., 1984).

The effects of photo-oxidation also facilitates the formation of stable water-in-oil emulsions (Thominette \& Verdu, 1984), some of which are extremely resistant to dispersion by physical or chemical means. In fact, several oils studied by Payne \& McNabb, Jr. (1985) could only form stable water-in-oil emulsions after simulated photochemical or microbial oxidation. The formation of stable water-in-oil emulsions along with the increase in viscosity from photo-oxidation, alters the spreading characteristics of the surface slick for a number of crude oils. Results indicated by Payne \& Phillips (1985a) show that viscous oils have different spreading characteristics than non-viscous oils, and may actually display a tendency to contract as opposed to continued spreading in the presence of photo-oxidative mechanisms. Decreases in the floating oil lense diameters of heavy crudes were observed with irradiation 
in as little as two hours, and were attributed to the inability of the photo-oxidation products formed at the oil-air interface to diffuse through the viscous oil layer and disperse through the oil-water interface (Payne \& Phillips, 1985a; and Klein \& Pilpel, 1974)

Several mechanisms have been proposed for the photo-oxidation of petroleum, including: (a) free radical oxidation in the presence of oxygen; (b) singlet oxygen initiation of hydroperoxide formation; and ground state triplet oxygen combining with free radicals to form peroxides (Payne \& McNabb, Jr., 1985). Reaction rates are directly affected by natural variations in cloud cover, atmospheric ozone, conditions affecting the transmittance of light in water, and the concentrations of humic substances (Larson et al., 1977; Payne \& McNabb, 1985, and Payne \& Phillips, 1985a).

The rate of oxidation is also proportional to the chemical nature of the compound, with more highly substituted molecules such as alkyl-substituted naphthenes being oxidized more readily than normal paraffins (Feldman, 1973; Berridge et al., 1968; Payne \& Phillips, 1985a, and Thominette \& Verdu, 1984). Additionally, the oxidation of aromatic compounds such as phenanthrene or anthracene, which yield quinones that are used as photosensitizers, may actually increase the efficiency of the photo-oxidation process (Andersson and Bobinger, 1992). 
Larson et al. (1977) determined that a Nigerian crude oil was not significantly affected by exposure to light and the photo-oxidation process was actually inhibited by the presence of a high sulfur content; he stated that these inhibition reactions are significant in the overall environmental photochemistry of some spills. Organosulfur compounds, particularly heterocyclic sulfides common in crude oils, are known to be effective inhibitors of radical reactions, even at low concentrations, and are readily converted to sulfoxides by hydrogen peroxide which effectively terminate the radical chain reaction initiated by photo-oxidation (Thominette \& Verdu, 1984; and Berrideg et al., 1968).

Johansson et al. (1980) conducted experiments and concluded by exposing oil to different types of light sources, that photo-oxidation of crude oil does not occur to any appreciable amount when exposed to wavelengths above $350 \mathrm{~nm}$; no degradation products are detectable even with exposure periods as long as 6 weeks. In spite of low quantum efficiencies at longer wavelengths, the total rate of decomposition to be expected in natural sunlight may be responsible for the decomposition of a 2.5 -um thick oil slick per 100 hours of sunlight (2.5 tons per square kilometer during this exposure period). The decomposition rate stated is for slicks with a thickness greater than $0.1 \mathrm{~m}$; thinner slicks are partially transparent to longer wavelengths and therefore have reduced destruction rates. 
Petroleum hydrocarbons observed to respond in wavelengths $310 \mathrm{~nm}$ or longer include acenaphthene, anthracene, B-methyl anthracene, and naphthalenes, along with nitrogen. oxygen, or sulfur-containing organics (Feldman, 1973). Polymerization and disproportionation can result in the formation of smaller molecules, along with longer chain higher molecular weight compounds, were stated to occur in wavelengths longer than 320 $\mathrm{nm}$; the intermediate steps of these type reactions which initiate from a polycyclic aromatic base can lead to carcinogenic by-products (Feldman, 1973).

\section{$\underline{2.5 \text { Emulsification }}$}

The two weathering processes which determine the ultimate fate of oil spilled on the sea, and the degree of environmental impacts associated from the oil spill, are vertical dispersion into the water column under the forces of wind and wave action, or emulsification (McAuliffe, 1976a). The fate is driven primarily by oil composition, spill location, meteorological conditions, and prevailing ocean conditions (Bobra et al., 1992; Exxon, 1989; and Buchanan \& Hurford, 1988). The formation of water-in-oil emulsions significantly reduces the rate of natural dispersion mechanisms and results in the formation of an extremely viscous residual material that persists on the surface of the sea (Lynch, 1987). The incorporation of water into the oil increases the volume of the polluted material and impedes the effective cleanup by mechanical recovery equipment (Bobra et al., 1992). The emulsion formed magnifies the clean-up effort and generally requires a labor intensive effort of removing the emulsion from the coastline by hand. 
The fractions of a given oil spill that evaporates, disperses into the water column. is degraded, or is left on the surface as mousse could depend critically on the exact sequence of light and wind/wave energy acting on the spill during the first few days (Thingstad \& Pengerud, 1983).

In a complex mixture such as petroleum, all these factors interact in such a way that all components of the mixture remain in the liquid oil phase (i.e., the lighter components of the mixture acting as solvents for the higher molecular weight molecules). As long as this solvency interaction continues and thermodynamic conditions remain unchanged, the oil remains stable. If the equilibrium point is changed, a point will be reached where the solvency strength of the oil is insufficient to maintain the heavy components in solution, and they subsequently precipitate out of solution (Bobra et al., 1992).

Indigenous petroleum emulsifying agents are concentrated in the higher boiling fraction of the crude, specifically in the component boiling above $370{ }^{\circ} \mathrm{C}$ and particularly in the residuum. Asphaltenes, resins, and waxes are believed to be the main constituents of the interfacial films that encapsulate the water droplets contained in the mousse (Bobra et al., 1992; Bridie et al., 1980; Berridge et al., 1968; Mackay et al., 1973). There is tremendous mechanical strength associated with these films which act as effective physical barriers, preventing droplet recoalesence. The stabilizing effect of the emulsion is solely dependent 
on the strength of these mechanical films and has no relation to any double laver interaction (Mackay et al., 1973).

Oil which reached the shore from the Amoco Cadiz spill was observed to be in the form of a water in oil emulsion. The volume and weight of this material which had to be removed from the beaches was substantially greater than the amount of oil alone, as much as five times in the case of "chocolate-mousse" (Huang, 1983; and Berridge et al., 1968), which is a persistent stable emulsion, highly viscous, with water content on the order of $70 \%$. Samples of the emulsion contained between $50-80 \%$ water (Wolfe, 1978), and contained varying amounts of clay and sand.

As the water content of the emulsion increases and concurrently degrades along the coastline due to continued weathering processes the physical properties of the oil become increasingly more difficult to handle. The initially inviscous free-flowing oil eventually becomes a relatively stable, heavy, non-pumpable material that must be either removed by hand or special equipment, or simply left on the beach (Wolfe, 1978; and Payne et al., 1983). As an example of the disparate physical properties between a parent oil and the final emulsion, an emulsion formed with $70 \%$ synthetic seawater and $30 \%$ Kuwait crude (fraction boiling above $200^{\circ} \mathrm{C}$ ) displayed thixotropic flow behavior, with viscosity measured in the range of 196,000 and $2,200,000 \mathrm{cP}$ depending on the applied shear rate. The viscosity of the $200+$ fraction of Kuwait crude at the same temperature was measured to be $490 \mathrm{cP}$ (Bridie et al., 1980). The 
initial rate of viscosity increase was shown to be dependent upon the asphaltene content of the oil (Lynch, 1987), and determines the window of opportunity for any counter-measure strategy, before highly viscous fluid properties pose handling difficulties.

Large lumps of emulsified oil eventually form impervious skins which effectively protect the oil from further weathering, and maintain the oil's buoyant condition for a long period of time (Davis \& Gibbs, 1975). Even in the presence of suitable populations of microorganisms in the water column, along with adequate amounts of mineral nutrients, oxygen and other components necessary to sustain microbial growth, the formation of stable emulsions precludes biological degradation, due to the sheltering of the bulk of the oil within the emulsions where conditions are unfavorable for microbial attack (Thingstad \& Pengerud, 1983; Patton et al., 1981).

The amount of energy required to form water-in-oil emulsions is quite small, and has been observed even in relatively gentle seas; therefore, the energy associated with breaking waves and spray induction must be many times that needed to form stable emulsions (Bridie et al., 1980; and Jeffery, 1981). In tests performed with various combinations of oil, wax content, and asphaltene percentage, it was determined that crude oil that has been de-asphaltenized does not form a stable emulsion; it was observed to shed $93 \%$ of its water content after 15 minutes, regardless if the original wax content was retained or concurrently removed (Bridie et al., 1980; and Berridge et al., 1968). The crude oil was only observed to form a stable 
emulsion when left in an unaltered configuration, or from an original oil composition with only $1 / 10$ of the original asphaltene content.

Asphaltenes are by definition high molecular weight heterocyclic organic compounds. These asphaltene molecules contain sufficient functional groups to penetrate the water phase boundary; yet the hydrophilic portion of the molecule is so large that it forms a layer on the oil side of the interface (Bobra et al., 1992). Waxes are simply high molecular weight alkanes.

The oil-water interfacial tension is an important parameter for predicting dispersion characteristics of an oil spill; this property is critical in performing an overall final mass balance for the slick. Asphaltenes, waxes, metallo-porphyrins (including vanadium complexes) (Berridge et al., 1968), and possibly the products of photochemical and microbial degradation, all are important in the stabilization of water in oil droplets by lowering the oilwater interfacial tension. Nitrogen, sulfur and organic compounds in the oil can act as natural surfactants, reducing the oil-water interfacial tension similar to chemical dispersants (Exxon, 1989). These materials act as surfactants which effectively prevent the separation of the oil water phase due to water-water micelle droplet coalescence in the continuous oil phase; under these conditions stable emulsions can be formed (Payne et al., 1983). The oxidative polymerization of aromatics and/or oxygenated aromatics during weathering might also 
result in the production of additional asphaltenic materials (Frankenfeld, 1973; and Thingstad \& Pengerud, 1983).

Betancourt \& McLean (1973) observed that asphaltene content was increasing faster than could be explained by losses of volatile, non-asphaltenic materials, and therefore some asphaltenes must have been produced; they suggested that the increase in asphaltenes is due in part to atmospheric oxidation, drawing parallels to the commercial production process of asphalt.

Bobra et al. (1992) noted that the stability of the emulsion formed was related to the alkane/aromatic ratio of the parent oil. For example, precipitation of the asphaltenes did not occur in an oil composed of $100 \%$ alkane, and the asphaltenes maintained their original size of approximately 1 micron, which is too small to effectively stabilize water droplets. There was a strong correlation for oils tested with a $50-95 \%$ alkane percentage for the formation of stable emulsions.

The effects of emulsification are to form rigid mats of oil material which are highly resistant to break up by surface turbulence. On the other hand, if emulsion flakes break off, vertical dispersion characteristics of the oil are enhanced because of the elevated densities of the emulsion which promote sinking. The oil density increases both because of evaporation of low boiling point constituents and the incorporation of water during emulsification. Few 
crude oils will weather sufficiently to sink alone; however, the density of many water-in-oil emulsions approaches that of seawater, and therefore, it requires very little uptake of particulate matter to sink it.

Temperature may also cause the floating oil to sink and resurface later (Buchanan \& Hurford, 1988). The final density of the emulsion depends to a great extent on the salinity of the water in which it was formed; therefore, mousse formed in high salinity waters would be expected to sink when drifting into less saline waters (Thingstad \& Pengerud, 1983).

\section{$\underline{\text { 2.6 Sinking/Sedimentation }}$}

Combined with the effects of vertical turbulent dispersion and depending on regional conditions, sedimentation of petroleum can be a significant factor which determines the ultimate fate of crude oil (Feldman, 1973). This factor can be of major importance when assessing the environmental impact of the oil spill on benthic communities. Most crude oils do not undergo weathering to the point where densities exceed that of seawater, but the sedimentation or sinking of oil can result from the increased density of water-in-oil emulsions generated as weathering proceeds; additional pathways include the incorporation into fecal pellets via zooplankton ingestion, adhesion to or flocculation of the oil with suspended particulate matter (SPM), incorporation of detrital material or sand during deposition on shore, or because of attached pelagic organisms (i.e., barnacles) (Payne \& McNabb, Jr., 1985; and Huang, 1983). According to Spaulding (1988), the sinking of oil 
through weathering alone is generally not encountered in colder northern waters: it has been observed however in Gulf of Mexico and Persian Gulf blowouts, depending on the bulk oil characteristics and the percentage of clay within the SPM population. The presence of microbes may also augment the tendency of the oil to sink, by increasing the density of the slick through the selective removal of lighter paraffins, or through the formation of agglomerates (possibly clathrates) with certain polynuclear aromatics. The biomass formed as organisms proliferate, provides a surface on which additional particulate matter collects, increasing the tendency of the mass to become negatively buoyant (Frankenfeld, 1973).

Oil spills in near shore waters with high suspended particulate loads experience rapid dispersal and removal of the oil due to sorption onto SPM along frontal zones (Payne \& McNabb, Jr., 1985). Experiments conducted by Payne \& McNabb, Jr., (1985), with simulated spills in outdoor wave tanks, revealed that at SPM concentrations from 1-10 mg/L, no appreciable transport of particle-associated oil to the sea-bed occurs. At SPM loads from 10-100 mg/L, considerable oil/SPM interactions with subsequent transport and deposition is possible in the presence of sufficient turbulent mixing, and at concentrations in excess of $100 \mathrm{mg} / \mathrm{L}$ massive oil transport may occur with the potential for significant adverse impacts to benthic communities. These results are consistent with typical SPM concentrations found in open ocean environments and estuarine areas, which generally fall within the range of 0.01 to $1.0 \mathrm{mg} / \mathrm{L}$, and 10 to $100 \mathrm{mg} / \mathrm{L}$, respectively (Delvigne et al., 1986). 
With higher energy dissipation rates found in coastal areas, it is evident that turbulence is important for oil-SPM interactions. The turbulence regime facilitates the formation of oil droplets from the coherent slick and the subsequent splitting of the oil droplets to suitable size ranges, and is the causal factor in coastal erosion and resuspension of bottom sediment. These two factors acting simultaneously in the same area increase the probability of particleoil droplet collisions. Oil droplet dispersion and sedimentation by oil-SPM interactions are more apt to occur before any appreciable weathering can alter the viscosity of the oil, because higher viscosities result in the generation of larger droplet sizes from the coherent slick which are less prone to sedimentation by SPM interactions. Additionally, once a stable water-in-oil emulsion has formed, further input of hydrocarbons to the water column and subsequent SPM adsorption is greatly reduced (Payne \& McNabb, Jr. 1985).

A comprehensive study of oil-SPM interactions is given by Payne \& McNabb, Jr. (1985). The interaction between dispersed oil and suspended particulates occurs either through the molecular sorption of the dissolved fraction or from the actual physical collision between oil droplets and suspended particulate materials. The collision of two particles in solution could result in particle destabilization and an overall reduction in the electric potential between the particles, due to compression of the electric double layer, decrease in surface charge, or particle bridging by substances in solution between the particles (Payne \& McNabb, Jr., 1985). It is also noteworthy to mention at this point that most suspended particles in solution, including the dispersed oil droplets carry a negative surface charge due to the 
dissociation of components into solution or from the selective adsorption of ions. The molecular adsorption of dissolved hydrocarbons to SPM is strongly correlated to the saltwater solubility of the individual species. Results of Gas Chromatographic studies performed by Payne \& McNabb, Jr. (1985) indicate that lower molecular weight aromatics and volatile aliphatic compounds will preferentially partition into the water column while the intermediate and higher weight aromatic and aliphatic hydrocarbons remained with the suspended particular material.

Zurcher \& Thuer (1978) pointed out that the identification of the dissolved, adsorbed, and colloidally dispersed oil fractions are significant in terms of assessing ecological impacts. The water soluble fraction has been identified as the one primarily responsible for acute toxicity, while the interruption of biological communication (i.e., chemotaxis) could possibly be linked to fractions adsorbed on particulates and accumulated in sediments. Herein lies the important relationship between the extent of crude oil weathering, the magnitude of vertical turbulent dispersion and the associated sedimentation of dispersed oil. Mayo et al. (1978) determined that the distribution of hydrocarbons in marine sediments subsequent to a major spill is strongly dependent on the extent of physical weathering encountered by the oil during the surface transport from the spill site to the area where final sedimentation occurs. This is important because once the oil penetrates into bottom sediments, further weathering is extremely limited, especially in cold anoxic sediments. 
The contribution of zooplankton to the overall sedimentation rate of crude oil was evaluated by (Freegarde et al., 1971). Research conducted using the zooplankton species Calanus finmarchicus estimates that the maximum rate of oil consumption by this species could be about $0.3 \mathrm{~g}$ per $\mathrm{m}^{3}$ of seawater per day. In the presence of an oil concentration on the order of $1.5 \mathrm{ppm}$, which is typical in choppy ocean conditions, an immobilization rate of 3 tons per day was estimated. This rate is contingent on a population of 2,000 individuals per cubic meter.

\section{$\underline{2.7 \text { Vertical Turbulent Dispersion }}$}

The weathering process which contributes the most to the overall disappearance of crude oil slicks spilled on the sea is the incorporation of discrete oil droplets into the water column under the driving forces of surface turbulence (Lynch, 1987). Vertical dispersion is defined as an entrainment process in which oil droplets are sheared from the coherent surface slick and subsequently entrained in the water column by such forcing mechanisms as breaking waves, downwelling surface currents generated by prevailing winds, or rain. Once dispersed in the water column, the final fate of the oil contained within these droplets depend on a number of processes, including: (1) potential interactions with suspended particulate materials which could transport oil to bottom sediments, imposing detrimental effects on benthic communities; (2) rapid dissolution of soluble hydrocarbons from the oil droplet due to an extremely large surface area to volume ratio associated with the droplets, which allows for toxic lower weight aromatic hydrocarbons, such as benzene and toluene, which are 
normally lost to evaporation, to enter the water column; (3) ingestion of dispersed oil by marine organisms which is ultimately deposited to bottom sediments sequestered within fecal pellets; and (4) long range transport by deep ocean currents which expands the area impacted by the spill.

The susceptibility of a surface slick to vertical dispersion, and the rate at which this process occurs, is a function of oil slick thickness which is dependent on: the degree of spreading; oil water interfacial tension; the characteristics of the oil, including density, viscosity, and the degree to which the oil is effected by weathering (Exxon, 1989); sea-state conditions, such as wave height, wave period, fraction of the sea surface encountering breaking waves (Huang, 1983; and Leech \& Walker, 1992); and meteorological conditions, such as wind, temperature and solar intensity.

The study of vertical dispersion mechanisms is important in assessing the potential environmental impacts to benthic communities, especially where high concentrations of suspended particulates are present, which increases the sedimentation of oil to the bottom. The quantification of vertical dispersion in the overall disappearance of the surface slick is important in assessing potential environmental impacts, especially in coastal areas rich in biodiversity, where toxic compounds can gain entry in food chains. The effects of vertical dispersion on the enhancing the spreading of oil was determined by Forrestor (1971). He studied the distribution of submerged oil particles in way of the tanker Arrow grounding by 
utilizing a modified plankton sampling gear towed through the water column. It was determined that oil concentrations relative to oil droplet sizes with effective lengths (i.e.. cube root of volume) in the range of 0.1 to $1.0 \mathrm{~mm}$ decreases with increasing depths. This is because of particle resurfacing forces which gain an edge on downwelling forces at deeper depths, resulting in droplet size distributions progressively shifting to smaller sizes with depth; oil consuming bacteria can also exert outside influence on the distribution of oil within the water column by preferentially degrading smaller droplets; concurrent chemical alterations also occur through preferential degradation (Atlas et al., 1981). With oil droplets less than $0.1 \mathrm{~mm}$ effective length, the total volume of oil did not show any decrease with depth. These oil droplets with negligible resurfacing forces become permanently incorporated into the water column. As an indication of the extent to which vertical dispersion affects the spreading of oil, positive traces of oil were detected at depths as large as 80 meters, which coincided with the deepest sampling undertaken.

As a general rule, droplets with diameters greater than $0.1 \mathrm{~mm}$ will return to the sea surface once the turbulence or surface agitation abates, while droplets with diameters below this critical diameter are considered to remain permanently incorporated in the water column (Payne \& McNabb, Jr., 1985; and Huang, 1983). The physical process of oil droplet formation results in the generation of sizes believed to fall within the range of 20 to $500 \mathrm{um}$ in diameter (Leech \& Walker, 1992). Studies carried out by Shaw (1976) determined the size distributions of particles and subparticles formed by hydrocarbons mixed with seawater 
under controlled laboratory conditions. As determined by a Coulter counter, the greatest number of oil particles was in the size $1-30$ um. Field data on droplet size distributions of dispersed oil is limited and encompasses a wide range of sizes. Berridge et al. (1968) recorded oil droplet sizes in the range of $20-40$ um in the presence of vigorous wave and current action; and Forrestor (1971) measured oil droplets in the surf zone adjacent to the grounded tanker Arrow in the range of $5-1000$ um. Experiments found in Delvigne et al, (1986) determined that mean droplet sizes were on the order of 100 um across a wide range of breaking wave conditions in simulation conducted in a laboratory flume. These droplets are not stable and will usually recoagulate unless diffused very quickly (Berridge et al., 1968). Conversely, these dispersions can be stabilized by surface active agents which can coat the oil-water interface, effectively preventing the oil from coagulating (Delvigne et al., 1986).

The factors which determine droplet size distribution are oil characteristics, turbulence intensity imparted to the surface slick, and turbulence duration. Experiments by Milgram et al. (1978) indicated oil thickness and oil viscosity have considerable effects on the fraction of dispersed oil, and that the penetration of oil droplets (including oil coated water droplets) into the water column is significant in the wake of a breaking wave. The breaking of a wave within the surface slick results in the injection of oil particles with a wide range of droplet sizes. Raj \& Griffiths (1979) studied the survival of oil slicks on the ocean as a function of sea state, to better understand the interaction between spilled oil and sea state, and to predict 
the dispersion and ultimate physical fate of oil spilled on rough water. His results indicate that a sea with a significant wave height of at least 3 meters is required before any globular vertical dispersion of oil is observed. He also determined that deep ocean non-breaking swells do not usually fracture a coherent oil slick, even though the energy associated with these type waves is more than sufficient to disperse oil droplets vertically into the water column. In the event of large scale oil spills, vertical dispersion is initially restricted by the large thickness of the slick, because of the effective dampening out of ocean waves passing through it, which results in the slick riding intact atop the large swells. Breaking of waves, except the very large ones are inhibited within the slick. Eventually, the slick becomes sufficiently thin from the effects of surface spreading, and becomes vulnerable to the effects of waves and upper ocean turbulence. A point is reached where the coherent slick is fractured by surface waves resulting in a shower of oil droplets being thrown down into the water column. The size distribution of these oil droplets depends largely on the properties of the crude oil, the thickness of the slick, and the energy dissipation rate of the ocean surface. Raj \& Griffiths (1979) defined the thickness where vertical dispersion of oil droplets becomes significant as being on the order of $5 \mathrm{~mm}$.

Lynch (1987) conducted laboratory tests with tanks and large free floating rings at sea. The mechanism by which oil is dispersed naturally is considered to be as follows: the oil spreads out to a thin enough layer (estimated to $1 \mathrm{~mm}$ or less). Once thin enough the oil layer will disperse, however the initial dispersion rate is highly dependent on the rate at which the 
viscosity of the oil increases as weathering proceeds, which is directly proportional to the asphaltene content carried by the parent oil. The dispersion rate is inhibited by an increase in viscosity, and will continue, albeit at a reduced rate until an emulsion is fully formed, whereby further dispersion is contingent on emulsion stability. It was also recorded from field data that total concentration of dispersed hydrocarbons was approximately ten times greater than the truly dissolved lower aromatic hydrocarbons.

In addition to the energy imparted to the slick by the bore of the breaking wave, there exists a turbulence wake that trails behind the wave that induces droplet splitting and vertical advection. As a criterion of whether the oil droplet will continue to split under the forces which are exerted upon it, Delvigne et al. (1986) lists the Weber Number as a means of evaluating this phenomenon. The oil droplets dispersed into the water column will continue to break up into smaller droplets until the dominant splitting forces, which are associated with the turbulent fluctuations in the water found in the wake of a passing wave, are again smaller than the dominant internal resisting force of surface tension (i.e., Weber Number $>1$ ). The tendency for droplet splitting is determined by the value of the Weber Number, and provides an indication of the minimum size of oil droplets to expect for a given set of input energy conditions and oil properties.

$$
W_{e}=T d /\left(\rho_{W} v_{d}^{2} d^{2}\right)=\text { surface tension / inertia force }
$$

where: 


$$
\begin{array}{ll}
\mathrm{W}_{\mathrm{e}} & =\text { Weber Number (dimensionless) } \\
\mathrm{T} & =\text { oil-water interfacial surface tension } \\
\mathrm{d} & =\text { droplet diameter } \\
\rho_{\mathrm{W}} & =\text { density of water } \\
\mathrm{v}_{\mathrm{d}} & =\text { rms velocity over distance } \mathrm{d} .
\end{array}
$$

In order to accurately quantify the extent of vertical dispersion and the effects of this process may have on other weathering processes (i.e., sedimentation or dissolution), and in a effort to predict environmental fate of oil spilled at sea, a means must be available for predicting oil droplet sizes and the depths to which these droplets are forced into the water column. Raj \& Griffiths (1979) predicts that the minimum diameter of submerged oil droplets, as a function of the surface turbulence in the presence of breaking waves, follows a relationship similar to:

$$
\mathrm{d}(\min ) \cong 0.12\left[\mathrm{~T}^{3 / 5} \omega^{2 / 5}\right] /\left[\rho_{\mathrm{w}}^{3 / 5} \mathrm{~g}^{4 / 5}\right]
$$

where:

$$
\begin{aligned}
\mathrm{d}(\mathrm{min}) \quad= & \text { minimum diameter of submerged oil droplets } \\
\omega & \text { modal wave frequency of the ocean spectrum } \\
& \text { (value of } 2 \mathrm{rad} / \mathrm{sec} \text { assumed for laboratory wave experiments) } \\
= & \text { oil surface tension } \\
\mathrm{T} & =\text { density of water }
\end{aligned}
$$

If the thickness of the slick is less than $\mathrm{d}(\mathrm{min})$ as calculated above, then smaller droplets can be formed and are predicted to follow the relationship: 


$$
\mathrm{d}(\text { thin }) \sim 0.3 \mathrm{~T}^{2 / 5} \omega^{4 / 15} \mathrm{~h}^{1 / 3} /\left[\rho^{2 / 5} \mathrm{~g}^{8 / 15}\right]
$$

where:

$$
\mathrm{h} \quad=\text { thickness of the film }
$$

A number of mathematical models were formulated by Aravamudan \& Marsh (1981), utilizing previously published data from other studies, which can predict droplet formation by braking waves with special attention given to determining minimum and maximum droplet sizes expected under different conditions; along with the vertical transport (i.e., penetration depth) of the oil droplets. A relationship was presented which depicts the maximum oil droplet size as being a function of the properties of the oil itself, and not in terms of ocean conditions. The theoretical maximum droplet size is given by the following equation:

$$
d_{\text {MAX }}=\left[\left(12 \sigma_{i}\right) / g\left(\rho_{w}-\rho\right)\right]^{1 / 2}
$$

where:

$$
\begin{array}{ll}
\sigma_{\mathrm{i}} & =\text { oil-water interfacial tension } \\
\rho_{\mathrm{w}} & =\text { water density } \\
\rho & =\text { oil density }
\end{array}
$$

The factors which determine whether these oil droplets return to the surface and recoalesce with the coherent surface slick are primarily droplet size distributions and the density 
differential between the oil droplet and the bulk solution. For constant oil density, the smaller the buoyant resurfacing forces associated with the particle, the deeper the intrusion depth of the droplet for a given turbulence energy.

The depth to which dispersed oil droplets penetrate the water column was determined in Delvigne et al. (1986) to be in the vicinity of twice the breaking wave height, with oil concentrations displaying a linear decrease with increasing depth immediately after the passage of the wave. From measurements made during small-scale and large-scale outdoor wave tanks an estimate of the intrusion depth was shown to follow the relationship:

$$
\mathrm{z}_{\mathrm{i}}(\mathrm{m})=[1.50 \pm 0.35] \mathrm{H}_{\mathrm{b}}
$$

where:

$$
\mathrm{H}_{\mathrm{b}} \quad \text { = height of breaking wave }(\mathrm{m})
$$

Modelling efforts conducted by Raj \& Griffiths (1979) to predict the intrusion depth indicates a penetration depth in the region of a fraction of a meter, with depth strongly linked to oil-water density differential and oil droplet size. These calculations underestimated the depth of intrusion due to the assumption that the entire quantity of energy contained within the wave was released as a quick pulse of energy in the bore of the breaking wave. Experiments conducted by other researchers, as reported in Raj \& Griffiths (1979), indicated that the maximum depth of intrusion was achieved long after the breaking wave event, and 
the driving forces responsible for this dispersion was the turbulent eddy currents located in the wake of the breaker. A relationship was provided which estimates the maximum depth of intrusion for oil droplets due to the forces of breaking waves.

$$
(Z)_{\operatorname{MAX}}=0.32 \mathrm{M} /\left[\rho_{w} c u_{t}\right]
$$

where:

$$
\begin{aligned}
& \text { (Z) } \max \quad=\text { maximum depth of intrusion } \\
& \mathrm{M} \quad \text { = the rate of loss of wave momentum per unit crest length } \\
& \text { approximated by }\left(10^{-2.3}\right) \rho_{w}\left[g^{3} / \omega^{4}\right] \\
& \text { c = wave phase speed } \\
& u_{\uparrow} \quad=\text { the terminal velocity of the drop which penetrates to depth }(Z)_{\text {MAX }} \text {. }
\end{aligned}
$$

The formula implies that for a given wave energy, the depth of intrusion is greater for smaller oil droplets. The depth of intrusion was estimated to be 100 meters for a 50 um oil droplet formed by the passage of a $15-\mathrm{m}$ long wave with a height of $2 \mathrm{~m}$.

As reported in Raj \& Griffiths (1979), the terminal velocity of these size oil droplets is roughly $0.15 \mathrm{~mm} / \mathrm{s}$, which requires a time of 8 days to reach the maximum depth. A residence time of this magnitude is significant from the standpoint of long-range transport of dispersed hydrocarbons and the expansion of the area impacted by the spill. The presence of significant turbulence in the wake of passing waves was also supported by laboratory flume tank experiments conducted by Milgram et al. (1978). With breaking waves stated to 
to be essential for the formation of discrete oil droplets, another relationship was provided for estimating the intrusion depth of oil droplets:

$$
\begin{aligned}
& \text { maximum depth of intrusion : } z_{\max }=0.06 \mathrm{H}^{2} \omega / \mathrm{W} \\
& \text { which is attained at time } t_{d}=z_{\max } / \mathrm{W}
\end{aligned}
$$

where:

$$
\begin{aligned}
& \mathrm{W} \quad \text { = terminal velocity of the oil droplet } \\
& \mathrm{H} \quad \text { = wave height, related to wind speed by } \mathrm{H}=0.283\left(\mathrm{U}_{\mathrm{w}}\right)^{2} / \mathrm{g} \\
& \omega \quad \quad \text { = wave frequency, related to wind speed by } \omega=0.13 \mathrm{~g} / \mathrm{U}_{\mathrm{w}}
\end{aligned}
$$

In the absence of large scale circulations, all oil droplets with a residual positive buoyancy will eventually return to the surface and recoalesce to form a film of oil. The duration time for recoalesence to occur was estimated to be on the order of twice the resurfacing time in the absence of turbulence (Delvigne et al., 1986). This is purely an idealistic case, for the ocean is rarely without turbulence and sub-surface advective currents. Large Langmuir circulation patterns in the water column set up by surface wind forces will generate large downwelling currents to carry oil droplets down to significant depths. Estimates of this downwelling velocity in the Langmuir circulation pattern has been reported to be approximately 0.8 percent of the wind speed (Delvigne et al., 1986).

One of the most comprehensive undertakings to examine the process of vertical dispersion of oil particles in the presence of breaking waves is presented in Minerals Management 
Service, OCS Study MMS 87-111 (1987). Experiments were conducted both on a small scale utilizing a vertical grid column to simulate submerged oil and suspended particulate interactions, and in large outdoor wave tanks simulating near-full-scale wave heights. The most significant results of these experiments yielded an empirical relation for the droplet size distribution and entrainment rate (dispersed oil mass per unit time), as a function of the oil type, oil layer thickness, breaking wave energy, and temperature. An additional relationship was also developed for the intrusion depth of oil droplets as related to the wave height. A wide range of energy dissipation rates were required to properly satisfy scaling laws in order to ensure that turbulent eddy structures in the models truly simulated field conditions. Adequate scaling of large turbulent eddy structures is required for the study of the diffusion process, while small turbulent eddy structures are necessary for analyzing particle interactions.

These processes require widely different energy dissipation rates (e), and therefore preclude any attempts at modelling these processes at the same time (Delvigne et al.,1986). Typical values of energy dissipation rates (e) in field conditions are:

$\begin{array}{ll}\text { deep ocean } & =10^{-4} \text { to } 10^{-2} \mathrm{~J} / \mathrm{m}^{3} \mathrm{sec} \\ \text { estuary } & =10^{-1} \text { to } 1 \mathrm{~J} / \mathrm{m}^{3} \mathrm{sec} \\ \text { surface layer } & =1 \text { to } 10 \mathrm{~J} / \mathrm{m}^{3} \mathrm{sec} \\ \text { breaking waves } & =10^{3} \text { to } 10^{4} \mathrm{~J} / \mathrm{m}^{3} \mathrm{sec}\end{array}$


Results from the dispersion experiments for defining expected oil droplet sizes, found in the water column as a function of viscosity and various energy dissipation rates (e), are summarized as follows:

$$
\mathrm{d}_{50}, \mathrm{~d}_{\max } \sim \mathrm{v}_{\mathrm{o}}^{0.34( \pm 0.05)}
$$

$d_{50}$ (mean droplet size, um) and $d_{\max }(u m)$ were dependent on the oil type, weathering state and temperature only as far as these parameters influence oil viscosity $\left(\mathrm{v}_{\mathrm{o}}, \mathrm{cSt}\right)$. This relationship is supported by experiments which were conducted for viscosities in the range of $8-220 \mathrm{cSt}$, but may be extrapolated into higher viscosities as long as the oil displays Newtonian behavior.

$$
\mathrm{d}_{50}, \mathrm{~d}_{\max } \sim \mathrm{e}^{-0.50( \pm 0.1)}
$$

This relation is stated to be valid in the high turbulence regime with energy dissipation rates in excess of $100 \mathrm{~J} / \mathrm{m}^{3}-\mathrm{sec}$, where the additional break up of oil droplets due to vertical shear on the rising buoyant droplets is negligible. Additionally, the oil droplet size distribution was found to be independent of salinity, but dependent on the duration of the turbulence. Measurements from small-scale experiments with turbulent durations from 5 to 600 seconds indicated that steady-state values of $\mathrm{d}_{\max }$ in high turbulent conditions were obtained for times greater than 5 minutes. From large-scale wave outdoor wave tank experiments that the maximum droplet size $d_{\max }$ in the water mass was actually independent of the break up process due to turbulence, but rather dependent on resurfacing parameters like intrusion 
depth $z_{1}$, rise velocity of the oil droplets W $\left(d_{o}\right)$ (as per Stokes Law), and the vertical turbulent diffusion coefficient $\epsilon_{\mathrm{z}}$.

The size distribution of oil droplets in the water column was also found to be independent of the oil layer thickness over the applied range of 0.2 to $1.2 \mathrm{~mm}$. As stated previously, it was determined by Raj \& Griffiths (1979), that the minimum oil layer thickness for initiating globular dispersion was $5 \mathrm{~mm}$, so it may be that droplet size distributions are independent of thickness below this critical value.

As for the entrainment rate of oil into the water column $(\mathrm{Q})$ with the passage of breaking waves, it was determined that the following proportionality existed;

$$
\mathrm{Q} \sim \mathrm{D}_{\mathrm{ba}}^{0.57( \pm 0.06)}
$$

where:

$$
\mathrm{D}_{\mathrm{ba}} \quad=\text { energy dissipation per unit surface area }\left(\mathrm{J} / \mathrm{m}^{2}\right)
$$

Dissipated energy values ranged from 1 to $9 \mathrm{~J} / \mathrm{m}^{2}$, in small scale experiments, to a value of 200 to $900 \mathrm{~J} / \mathrm{m}^{2}$ for large wave flumes. The entrainment rate was determined to be independent of the surface oil layer thickness, and also independent of water salinity. The break up of the oil slick into discrete droplets in the water column was also found to be dependent on the oil-water density differential, oil-surface tension, and oil-water interfacial tension. Modeling conducted by Huang (1983) on the break up of oil slicks in way of 
breaking waves, assumed that the volume of the entrained oil increases linearly with sea state and oil to water density ratio, and will decrease linearly with increasing oil-to-water interfacial tension.

Finally, dispersion of oil droplets can occur spontaneously through the formation of micelles by physical/chemical processes. As defined in Delvigne et al. (1986), a micelle is an agglomeration of surface active compounds comprised of long chain hydrocarbons molecules with polar functional groups at one end. The hydrocarbon part of the molecule will assemble in the center of the molecule, while the hydrophilic polar groups remain on the periphery of the cluster in contact with the bulk water phase. In this configuration, normally insoluble hydrophobic hydrocarbons are solubilized. The stability of the micelle in suspension is maintained by a net surface charge leading to repulsive forces. In the presence of electrolytes (i.e., salts contained within seawater), destabilization takes place resulting in coagulation. The coagulation could take place by collisions between particles due to different rising velocities across the particle size spectrum, velocity gradients in the water phase, or Brownian motion. This coagulation of particles has been found to influence particle size distributions. 


\section{EXPERIMENTAL OUTLINE}

Three grades of crude oils were provided directly by MOBIL Oil Corporation for analysis which encompassed a wide range of API specific gravities that can be expected in worldwide trade. The oils available for analysis were the Statfjord Crude (Norway), Arabian Heavy Crude (Saudi Arabia), and the BCF-17 Crude (Venezuela). The API gravities of these crude oils are nominally 38,28 , and 17 , respectively; they are listed in order of ascending oil viscosity. The Arabian Crude oil was selected for analysis, because it maintains a middle ground ranking in terms of physical and chemical properties, i.e., oil viscosity, percent aromatics, and sulfur content. A comparison of the three crude oils, along with distillation data for the Arabian Crude, can be found in Appendix A. These properties are directly linked to the vertical turbulent dispersion properties of the oil, the dissolution potential, the subsequent toxicity potential of the oil to the marine environment, and the propensity of the oil to undergo photo-oxidative alterations. The main focus of this research is first to enhance the data compiled to date, by reproducing the time variant alterations in the physical properties of the oil as a function of the weathering period (i.e., oil viscosity, oil-water interfacial tension, and oil density); and second, to relate these properties to the associated vertical dispersion characteristics of the oil, as a function of the physical properties that exist at the end of the weathering period. 
The weathering of crude oil is the result of a series of a complex. interactive, and oftentimes competitive physical, chemical, and biological processes. These major weathering processes. previously identified, can be further classified into two major groups: those that alter the geometric configuration and geographic location of the slick, and those which reduce the mass of the slick and alter the chemical configuration of the oil.

The weathering of crude oil results in series of changes to the original physical properties of the oil. The oil will experience an increase in viscosity, largely dependent on the composition of the oil, because of the evaporative loss of lighter weight hydrocarbon compounds. A reduction in the oil-water interfacial surface tension will occur due to the increase in concentrations of natural surface active materials within the oil as the lighter hydrocarbons are lost, in conjunction with the formation of additional surface active materials from photo-oxidative processes, and the entrainment of water. Finally, an increase in the density of the oil will be observed, because of evaporative losses and the entrainment of water associated with emulsification. These physical characteristics have a significant effect on the vertical dispersion characteristics of crude oil, including: formation and size of oil droplets, resurfacing velocities of entrained oil droplets in the water column, subsequent recoalescence rate of the oil droplet upon returning to the surface, and the propensity of the entrained oil droplets to sink. In addition, oxidative processes, initiated by sunlight, have been linked to chemical alterations in the parent oil, which result in an increase in the toxicity of the oil due to the formation of more soluble intermediate products, which may increase 
the environmental impacts in sensitive areas. especially in tropical and sub-tropical regions similar to south Florida. Therefore, the experiments conducted in conjunction with this research effort have been designed in such a way as to include the monitoring of the physical properties of the oil ( i.e., viscosity, density, oil-water interfacial surface tension), as a function of the weathering period; to compare the collected data with previously published information, and to relate the resultant changes in these properties, due to weathering, to the vertical dispersion characteristics of the oil in a laboratory scale turbulence simulator.

The variation in the physical properties of the oil as a function of the weathering period were tracked by utilizing a Brookfield Digital Cone Plate Viscometer (Model LVDV-II++) equipped with a $\mathrm{CP}-40$ spindle and a jacket water cooled sample cup, designed for measuring oil viscosities in the range of 0.3 through $1028 \mathrm{cP}$. A Fisher Scientific du Nouy Ring apparatus (Surface Tensiomat Model 21) was used for oil-water interfacial surface tension measurements, and a calibrated $10-\mathrm{mL}$ pycnometer and analytical balance for recording density variations. The characterization of the dispersed oil samples extracted from the simulator was performed utilizing a Mettler AE 240 analytical balance for quantification of total oil concentration; a Gilford Fluoro IV Spectrofluorometer for quantification of total aromatic hydrocarbons, including compounds identified as having known toxic effects on marine organisms; and a Hewlett Packard Model 5890 Gas Chromatograph/Mass Spectrometer (GC/MS) for identifying chemical compounds and tracking chemical alterations due to weathering processes. 
Associated average colloidal particle size and particle charge distributions within the water column were performed by a Coulter Doppler Electrophoretic Light Scattering Analyzer (DELSA 440). This device utilizes electrophoresis techniques and has the ability to scan for particles in the sub-micron range (0.02-30um). Oil droplets in the colloidal fraction range may have important relationships to the overall toxicity of oil spill dispersion, the persistence of the oil suspensions in the water column, and are also identified as the size range most susceptible to long-range dispersion by sub-surface currents. The relative toxicity of the weathered oil samples was therefore characterized by a Microtox 500 Analyzer, in an effort to ascertain the time dependent relationship between the total aromatics present in the aqueous phase, a function of the weathering period, and the relative toxicity of the spill.

It is noteworthy to mention that the Arabian Crude oil was delivered directly from the MOBIL refinery to the Environmental Laboratory of Florida International University in a sealed 1 gallon can. The oil required for this analysis was subsequently transferred from the gallon can to ten (10) 300-mL amber glass bottles, and tightly sealed with no headspace prior to conducting experiments. The ten glass bottles were suitably stored in a cool dark place until needed. This procedure was deemed necessary in order to limit the variance in the initial conditions of the oil between experimental trials. 


\section{EXPERIMENTAL PROCEDURES}

\subsection{Weathering Characteristics}

This section was designed to track the change in the physical properties of the surface slick shown to have a strong correlation to the vertical turbulent dispersion characteristics of the oil, including evaporative loss, viscosity, oil-water interfacial surface tension. and density. Data was acquired as a function of the weathering period, which was recorded as the exposure time to weathering action, and in terms of the total solar irradiance recorded during the respective exposure period. Total solar irradiance across the spectrum range of 380-1100 nm was recorded by a LI-COR Pyranometer Sensor (LI-200SA), which was linked to a data logger, to provide an indication of the relative magnitude of solar intensity during the exposure period. The data logger was programmed to record solar intensity at 5 second intervals and to compute an hourly average intensity, which was then recorded in permanent memory for later retrieval at the end of the weathering period. The LI-200SA is a fully cosine-corrected sensor, with a typical cosine error of less than $5 \%$ up to an $80^{\circ}$ angle of incidence. The silicon photodiode was calibrated, by the manufacturer, against a working quartz halogen lamp acceptable to the National Bureau of Standards (NBS). The selected duration of the weathering exposure for the oil samples was, 4,8 , and 24 hours; plus 4 and 7 days. Additionally, the initial physical properties and dispersion characteristics of the oil were determined from an unweathered oil sample. 
Data was also acquired during this phase to quantify the concentrations of "soluble" hydrocarbons beneath the surface slick as a function of the exposure period. In addition, changes in the chemical composition of the weathered oil were identified by comparing the GCMS spectral data of weathered and unweathered oil samples.

A crude oil weathering station was constructed as shown in Figure 2 to support the weathering flask, and to house a magnetic stirring plate and electric Whisper 200 aquarium type air pump. The air pump provided sufficient air supply to allow for an unrestricted evaporation rate during the weathering period. The weathering flask consisted of a $2000-\mathrm{mL}$ Pyrex ${ }^{\circledR}$ Erlenmeyer flask with a specially constructed rubber stopper configured to accept a $10-\mathrm{mL}$ burette tower along with an air supply inlet and exhaust fitting. The weathering flask was filled with artificially prepared seawater to the $1200-\mathrm{mL}$ marking. The seawater was prepared using Tropic Marin $®$ Sea Salt to a specific gravity of 1.022 using regular tap water.

Prior to admitting the crude oil from one of the $300-\mathrm{mL}$ amber glass sample bottles, the entire weathering flask assembly, which included the $1200-\mathrm{mL}$ of seawater, a 1-1/4" magnetic stirring bar, the rubber stopper; and the $10-\mathrm{mL}$ burette tower, was suitably preweighed using an analytical balance. After recording the initial weight, the entire contents of the $300-\mathrm{mL}$ glass bottle was transferred to the weathering flask using a long neck plastic funnel. 


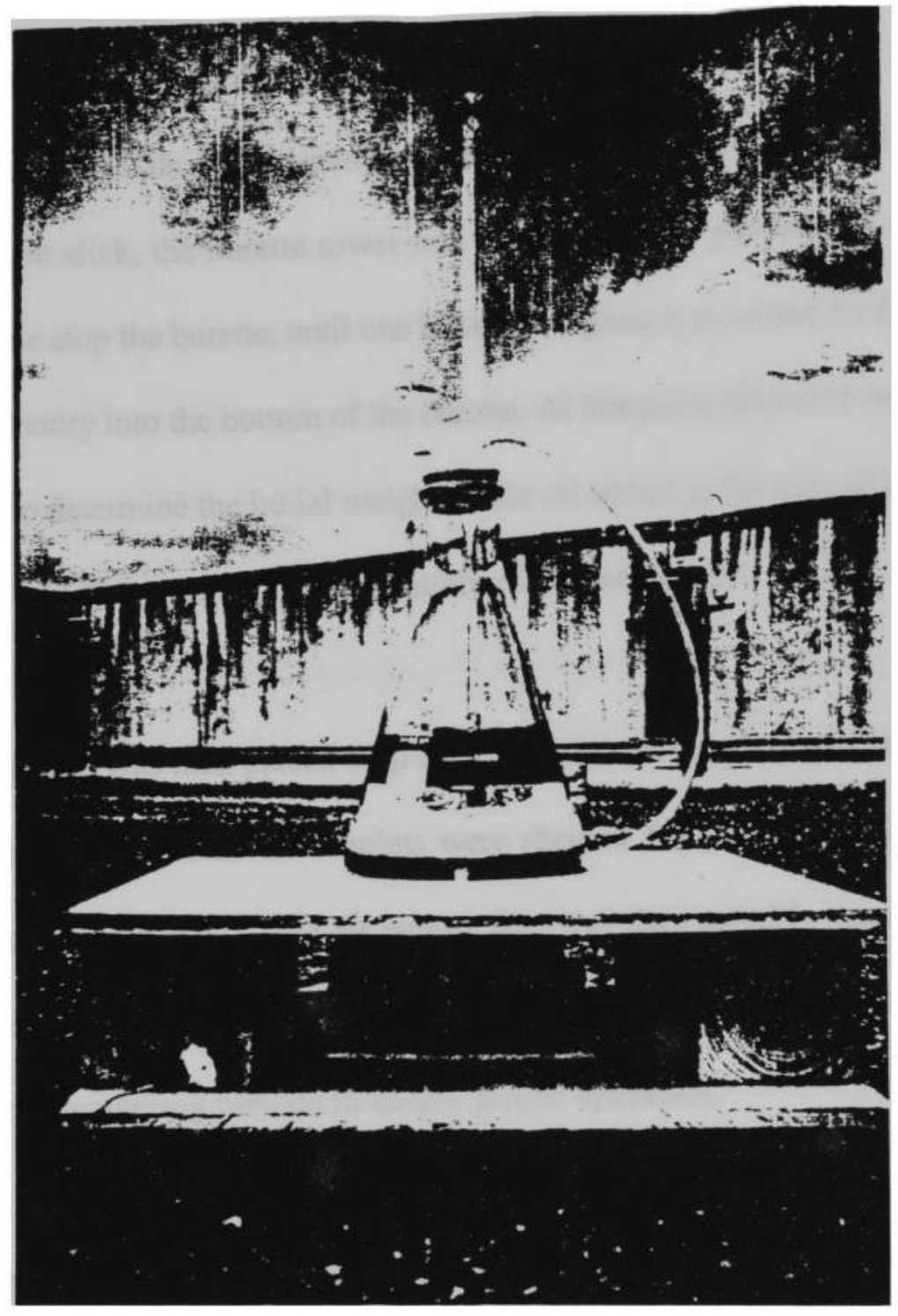

Figure 2

Crude Oil Weathering Station 
The rubber stopper was lifted slightly and the burette tower shifted to one side of the flask mouth to permit insertion of the funnel. Extreme caution was exercised at this point to ensure that the end of the burette remained well below the water surface, and that the oil was added slowly enough so that oil droplet formation in the water phase was minimized. Once the oil formed a coherent slick, the burette tower was back purged by placing the air supply line over the vent hole atop the burette, until one bubble had passed, to ensure no fine dispersions of oil had found entry into the bottom of the burette. At this point the entire weathering flask was reweighed to determine the initial weight of the oil added at the start of the weathering period, and the LI-COR data logger was initialized and activated.

The weathering flask was then placed atop the magnetic stirring plate and set at a very low mixing level to ensure that no oil droplets were sheared from the underside of the slick during the weathering period. The air supply line was then suitably connected atop the weathering flask and proper flow checked. A check of the system was made twice daily during extended weathering periods to ensure proper operation.

Upon completion of the weathering period, the air supply line was removed from the weathering flask and the entire assembly reweighed to ascertain the percent of crude oil weight lost due to evaporation. 
It was assumed that the oil slick. with a initial thickness of approximately $1 / 2 "$. adequately prevented the evaporative loss of water, and that the entire weight differential recorded was attributed solely to the loss of lighter molecular weight hydrocarbons from the oil sample.

After recording the post weathering weight, two (2) $250-\mathrm{mL}$ volumes of water were withdrawn from below the slick directly into individual $250-\mathrm{mL}$ separatory funnels. The burette tower was flushed with two volumes of water to remove any possible contamination prior to sample collection. The $250-\mathrm{mL}$ water samples were immediately treated in situ with approximately $15 \mathrm{~mL}$ of methylene chloride to fix the aqueous phase and then were promptly transferred to the laboratory for completion of the extraction procedure. As a protocol for all extraction procedures the samples were suitably extracted with three (3) $15-\mathrm{mL}$ aliquots of methylene chloride.

All extracts were transferred to a $100-\mathrm{mL}$ volummetric flask and distilled with methylene chloride to volume, two (2) 11-mL capacity glass sample vials were then filled leaving no headspace, and immediately transferred to a freezer maintained at $-20^{\circ} \mathrm{C}$. After collecting two aqueous phase samples, the burette tower was completely removed from the weathering flask, and a 50-mL oil sample was withdrawn from the slick by pipette for physical characterization. Utilizing a $10-\mathrm{mL}$ pipette, the oil sample was extracted from the flask with special attention given to obtaining the best representative sample. This was achieved by collecting oil at a number of locations around the oil slick, and transferring the aliquots to 
a $50-\mathrm{mL}$ borosilicate glass vial leaving no headspace. Oil samples were tightly sealed and allowed to settle for a 24-hour period in a cool dark place prior to running any tests to allow for the sample temperature to stabilize at a relatively constant value of $25^{\circ} \mathrm{C}$, and to visually check for the presence of water.

In addition, a small aliquot (approximately $0.5 \mathrm{~mL}$ ) of oil was removed from the slick. dissolved in methylene chloride within a glass vial, tightly sealed, and placed in a freezer for later analysis by GCMS. Finally, a $100-\mathrm{mL}$ volume sample of the remaining oil was carefully removed from the weathering flask by pipette, and transferred into a 250 -mL glass beaker for continuing analysis in procedures outlined in section 4.2 Vertical Turbulent Dispersion Characteristics.

The physical characterization of the oil was performed in the following fashion:

4.1.1 Oil Viscosity This parameter was determined by the use of a Brookfield Digital Cone Plate Viscometer (Model LVII++). Oil viscosity was measured using a precision torque meter checked for proper calibration using viscosity standards available from the manufacturer. Prior to conducting a viscosity measurement, the gap between the cone and the plate was mechanically adjusted to a gap clearance of $0.0005 "(0.013 \mathrm{~mm})$; see Figure 3, as per specific instructions contained within the operators manual. The adjustment of the gap 
clearance was performed after ensuring proper circulating water flow, and allowing 10 minutes for the sample cup to equalize at the set temperature of $25^{\circ} \mathrm{C}$.

In the absence of a controlled temperature water circulation unit, a cooling water circuit was configured directly from the cold tap water supply line. Water discharging from the sample cup was fed directly into a $1000-\mathrm{mL}$ beaker where a thermometer recorded the output temperature. All viscosities were recorded at $25^{\circ} \mathrm{C} \pm 1.0^{\circ}$.

The torque measuring system within the unit, consisted of a calibrated beryllium-copper spring connected in-line between the driving mechanism and the rotating cone. The resistance to rotation associated with the presence of fluid in the gap between the spindle and the stationary plate produces a torque that is proportional to the shear stress in the fluid, which is then converted to units of centipoise (cP) (refer to Appendix B).

The viscosity of the oil sample was measured at spindle speeds of $0.3,0.5,0.6,1.0$, and 1.5 $\mathrm{rpm}$, in order to provide the most representative analysis. A weighted average viscosity was calculated using the viscosity data output from an individual run combined with the percent of maximum scale reading for viscosity that the unit has for each particular operating speed. In addition, data was recorded and suitably plotted for shear rate versus shear stress at each operating speed, in order to ensure that a linear relationship existed, indicative of the fluid behaving in the Newtonian range (i.e., fluid viscosity independent of spindle speed). 


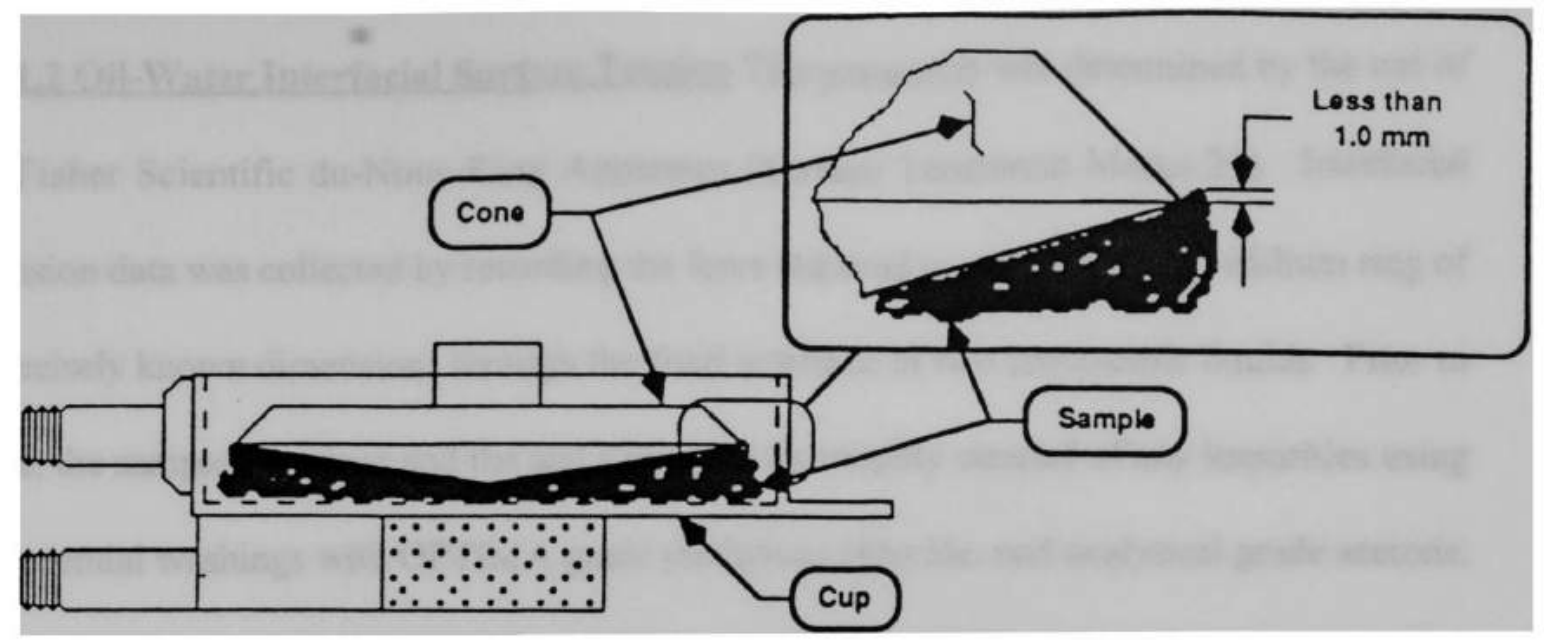

Figure A8

Notes: a) The cup may be removed without resetting the gap.

b) Remove the spindle from the Viscometer when you clean it.

c) Reset the hit point every time the spindle is attached.

\begin{tabular}{|l|c|c|}
\hline Cone & Sample Volume & Cone Angle \\
\hline CP-40 & $0.5 \mathrm{ml}$ & $0.8^{\circ}$ \\
CP-41 & $2.0 \mathrm{ml}$ & $3.0^{\circ}$ \\
CP-42 & $1.0 \mathrm{ml}$ & $1.565^{\circ}$ \\
CP-51 & $0.5 \mathrm{ml}$ & $1.565^{\circ}$ \\
CP-52 & $0.5 \mathrm{ml}$ & $3.0^{\circ}$ \\
\hline
\end{tabular}

Figure 3

Brookfield Viscometer Cone Plate Gap Clearance

and

Correct Sample Volume Verification 
4.1.2 Oil-Water Interfacial Surface Tension This parameter was determined by the use of a Fisher Scientific du-Nouy Ring Apparatus (Surface Tensiomat Model 21). Interfacial tension data was collected by recording the force required to pull a platinum-iridium ring of precisely known dimensions through the fluid interface of two immiscible liquids. Prior to use, the sample container and the test ring were thoroughly cleaned of any impurities using sequential washings with OPTIMA grade methylene chloride, and analytical grade acetone. followed by a final rinsing with distilled water. To conduct a measurement, the glass container was first placed atop the adjustable table ensuring that the table was initially placed at the maximum height setting using the worm gear adjusting ring. The entire table assembly was then lowered, by freeing the clamping mechanism on the vertical tower inside the case, to provide sufficient clearance to set the ring in place. The test ring was then suspended from the counter-balanced lever-arm; without releasing the locking mechanism holding the arm, and the more dense liquid added to the sample container to a depth of approximately $1 / 2 "$. Distilled water was used for the analysis to eliminate any possible error stemming from the reproducibility of laboratory prepared seawater samples, and to limit the corrosive effects of saltwater on the apparatus.

The entire table assembly was then raised until the ring immersed about $1 / 4$ " and was completely wetted by the heavier liquid. The locking mechanism holding the lever arm was then released and the unit adjusted for a zero reading. The adjustment for zero is accomplished by rotating the arm on the right side of the case, which thereby alters the 
torsion in a stainless steel wire controlling the vertical position of the lever arm. The adjustment is completed when the index and its reflected image are aligned with the reference mark on the mirror, and the indicator dial set to zero.

The vertical position of the table may need to be corrected to ensure that the ring does not come in contact with the bottom of the glass container or the surface of the liquid. Once the unit is in the zero position, the crude oil sample was poured atop the surface of the distilled water to a depth of $1 / 2$ " to $5 / 8$ ". The layer of the lighter liquid must be of sufficient depth to prevent the ring from coming in contact with the surface of the lighter liquid (i.e., air-oil interface) prior to rupturing the interfacial film. In accordance with procedures set forth in ASTM-D-971 the interface was allowed to stabilize or "age" for 30 seconds before conducting an actual measurement.

The sample table is slowly lowered by utilizing the worm gear adjustment and the test ring raised using the control knob on the side of the case. These alternating incremental adjustments are conducted in such fashion so as to maintain the position of the index at the reference mark. The interface between the two liquids will become distended (Figure 4) and will eventually rupture. The dial reading on the face of the unit at the breaking point of the interfacial surface is the "apparent interfacial tension" (P). The absolute value of the interfacial tension (S) is obtained by correcting the apparent value using the following formula: 


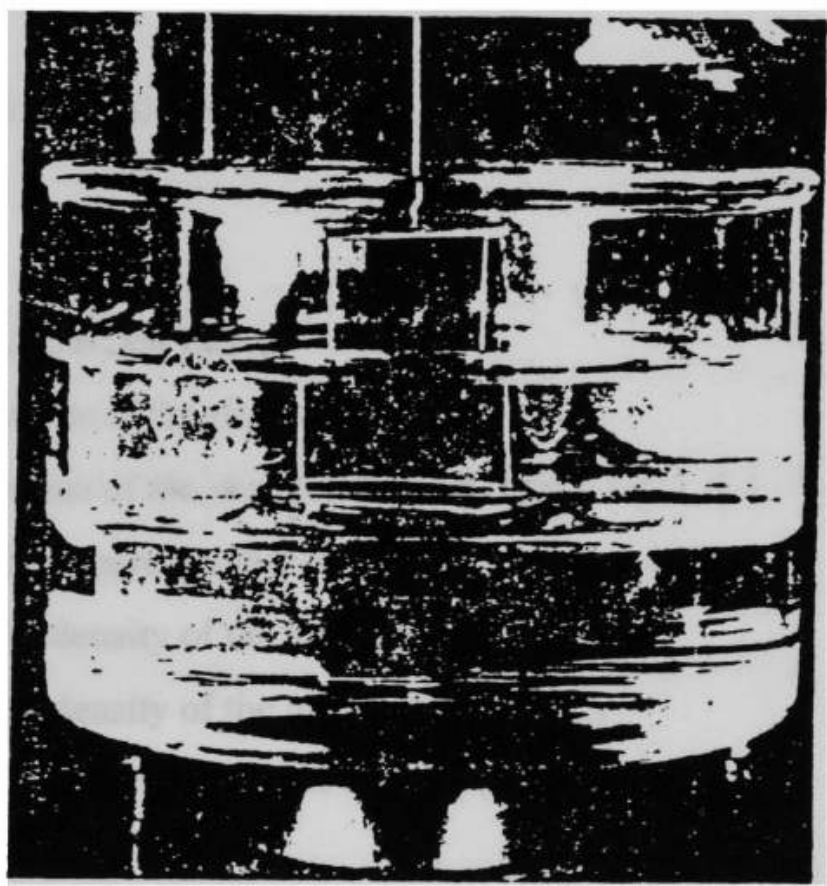

Figure 4

Distention of the Interfacial Surface Between Two Immiscible Liquids 


$$
\mathrm{S}=\mathrm{P} \times \mathrm{F}
$$

The parameter $(\mathrm{F})$ is a correction factor which is dependent on the size of the ring and the size of the wire used in the ring, the apparent surface or interfacial tension, and the densities of the two phases.

$$
F=0.7250+\left[\left[0.01452 P /\left[C^{2}(D-d)\right]+0.04534-[1.679 r / R]\right]^{0.5}\right.
$$

where:

$$
\begin{array}{ll}
\mathrm{F} & =\text { correction factor } \\
\mathrm{R} & =\text { radius of the ring }(\mathrm{cm}) \\
\mathrm{r} & =\text { radius of the wire for the ring }(\mathrm{cm}) \\
\mathrm{P} & =\text { the apparent value or dial reading }(\text { dynes } / \mathrm{cm}) \\
\mathrm{D} & =\text { the density of the lower phase }(\mathrm{g} / \mathrm{mL}) \\
\mathrm{d} & =\text { the density of the upper phase }(\mathrm{g} / \mathrm{mL}) \\
\mathrm{C} & =\text { the circumference of the ring }(\mathrm{cm})
\end{array}
$$

The physical constants of the platinum-iridium ring are $\mathrm{C}=5.985 \mathrm{~cm}$ and $\mathrm{R} / \mathrm{r}=53.6145350$. The calibration of the unit and of the experimental procedure was checked just prior to each weathered oil analysis by measuring the interfacial tension of hexane-water (51.1dynes/cm).

4.1.3 Oil Density This parameter was determined by the use of a $10-\mathrm{mL}$ glass pycnometer. A precision glass pycnometer with a volummetric capacity rating of $10-\mathrm{mL}$ at $25^{\circ} \mathrm{C}$ was used in tandem with an analytical balance to accurately record the density of the weathered oil samples. The pycnometer was calibrated by initially measuring the density of distilled water 
(density $=0.997 \mathrm{~g} / \mathrm{mL} @ 25^{\circ} \mathrm{C}$ ) in order to determine the calibration constant of the pycnometer vial. The density of the oil samples were determined by filling the pycnometer vial with oil to the indicated mark. When the vial cover is placed in position. all fluid in excess of the rated volumetric capacity of the vial will be forced out through a pilot hole machined into the cover. The weight of the pycnometer filled with $10 \mathrm{~mL}$ of sample was then compared against the weight of the pycnometer alone in order to determine the density of the sample. This calculated density of the sample was then corrected using the calibration constant determined for the vial.

\subsubsection{Molecular Characterization by Gas Chromatography/Mass Spectrometry}

(GC/MS). This phase of the characterization was accomplished by the use of a HewlettPackard Model 5890 Gas Chromatograph/Mass Spectrometer. At the end of each weathering period, small aliquots $(<1 \mathrm{~mL})$ of crude oil were removed from the surface slick contained in the weathering flask, and dissolved in methylene chloride. Small extraction columns were prepared using disposable 23-cm Pasteur pipettes filled with approximately $0.75 \mathrm{~g}$ activated Fisher Scientific Chromatographic Silica Gel (100-200 mesh). A 1-mL sample of the dissolved crude oil sample was placed into the top of the extraction column and the aliphatic and aromatic hydrocarbons were recovered by elution with OPTIMA grade methylene chloride. Approximately $5-\mathrm{mL}$ of solvent was required to accomplish this objective. The collected sample was blown-down under a light steady stream of nitrogen, to a volume of 
approximately $50-\mathrm{uL}$. A 1-uL sample was withdrawn from the sample vial and injected into the GC/MS for qualitative analysis.

The Hewlett-Packard GC/MS was fitted with a $30-\mathrm{m}$ long by $0.25-\mathrm{mm}$ internal diameter ®Durabond 5\% phenyl methylpolysiloxane column for the chromatographic separation of the samples. The temperature of the column was programmed from $40{ }^{\circ} \mathrm{C}$ to $300{ }^{\circ} \mathrm{C}$ at a rate of $6 \% \mathrm{~min}$, one minute after injection of the sample. The abundance of selected aliphatic and aromatic compounds were recorded; formed into a ratio of the respective compounds to the $\mathrm{n}-\mathrm{C} 30$ alkane, and a comparison made between different weathered oil samples, to formulate a relative loss rate for each of the selected compounds.

\subsection{Vertical Turbulent Dispersion Characteristics}

Vertical dispersion profiles for the weathered oil samples were characterized utilizing a specially designed and constructed vertical turbulent dispersion reactor, Figures 5-7. The reactor is constructed of 12 " diameter clear acrylic plastic (1/4" wall thickness) and stands at a height of $72 "$. The top cover plate of the reactor supports an internal 4" diameter acrylic tube, which houses the 3 " diameter mechanical mixing head, designed to serve as the turbulence generator. The speed of the mechanical mixing head can be adjusted up to 2500 rpm to vary the energy input rate to the reactor. Refer to Appendix $\mathrm{C}$ for a listing of the energy input rates calculated for a range of operating speeds for the mixing head, and a 
comparative listing of published energy dissipation rates commonly associated with various levels of surface turbulence in actual field conditions.

The internal 4" diameter tube projects 12 " into the main body of the reactor and is fitted with three inlet ports which allow water to cascade in from the main body of the reactor into the turbulence generator. This configuration serves to concentrate the turbulence energy in a confined area, and to deliberately restrict the water flow on the low pressure side of the propeller to induce cavitation. The air induction associated with cavitation is intended to simulate the entrainment of air after the passage of breaking waves in actual field conditions. The configuration of the upper workings of the reactor is also intended to separate the surface of the slick into a turbulent area and a relatively quiescent area to allow for any water-in-oil emulsification to become visibly evident. Vertical baffles adjacent to the inlet ports guide the oil into the turbulence generator during operation. The design waterline of the reactor is situated at the center of the inlet ports to the turbulence generator when the mechanical mixing head is at the chosen speed setting, which results in a operating volume for the reactor of approximately 30 gallons. Within the reactor, samples of weathered crude oil were exposed to turbulent surface conditions, designed to simulate an energy dissipation rate for surface turbulence commonly found in a deep ocean environment approaching breaking wave conditions. Water samples containing dispersed crude oil were withdrawn from the reactor at specified time periods from three (3) sampling ports along the depth of the reactor. 


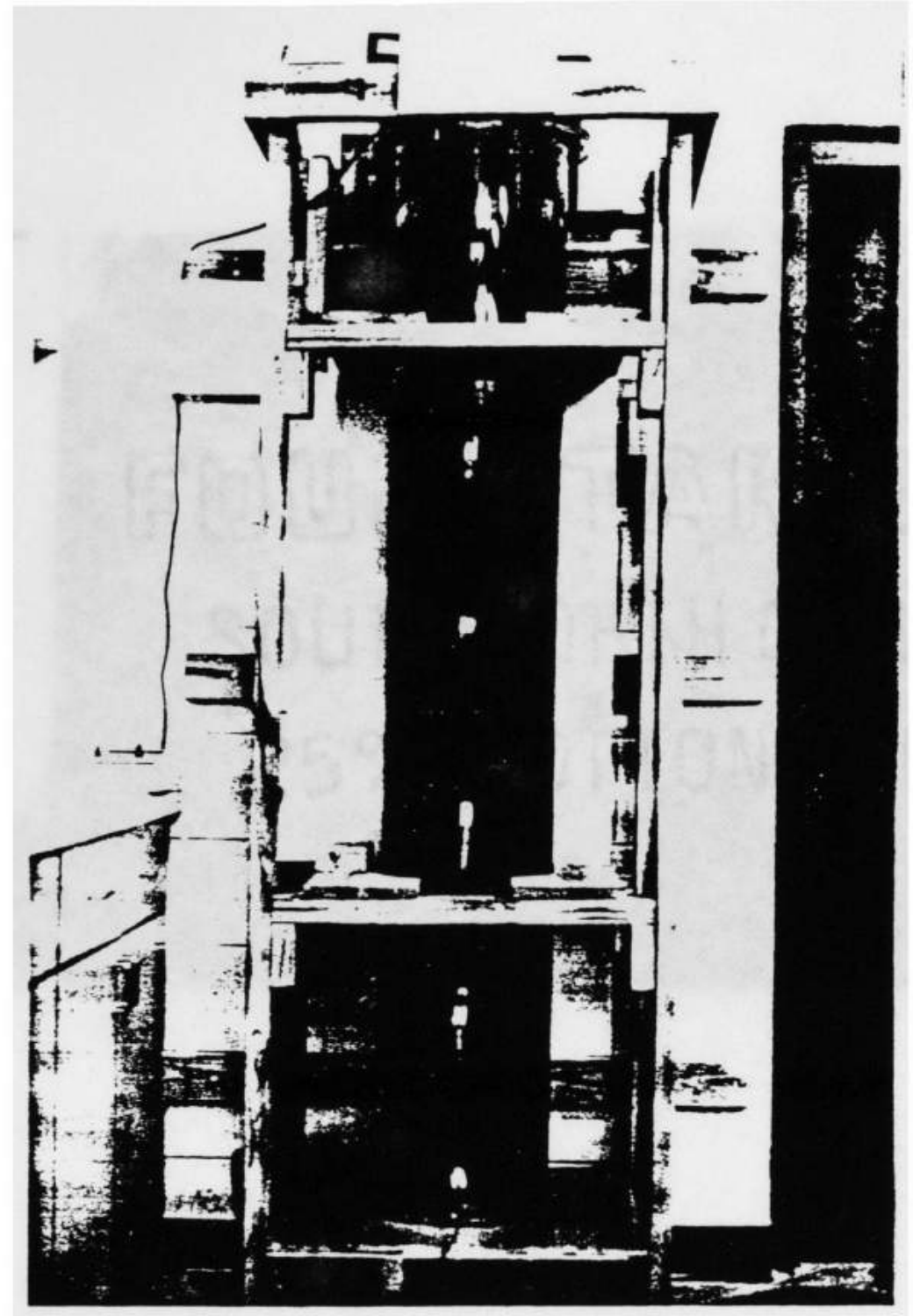

Figure 5

Vertical Turbulent Dispersion Reactor 


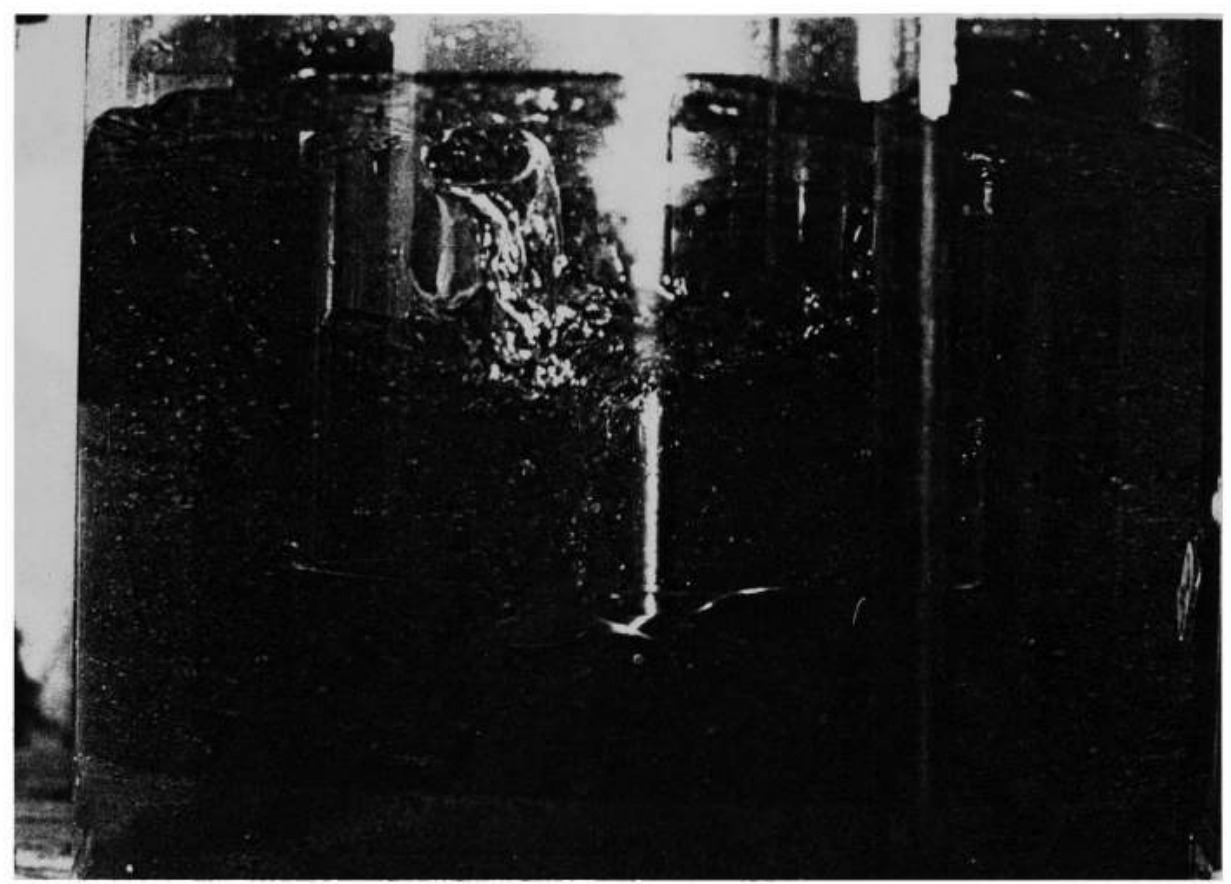

Figure 6

Internal 4" Turbulence Generator 


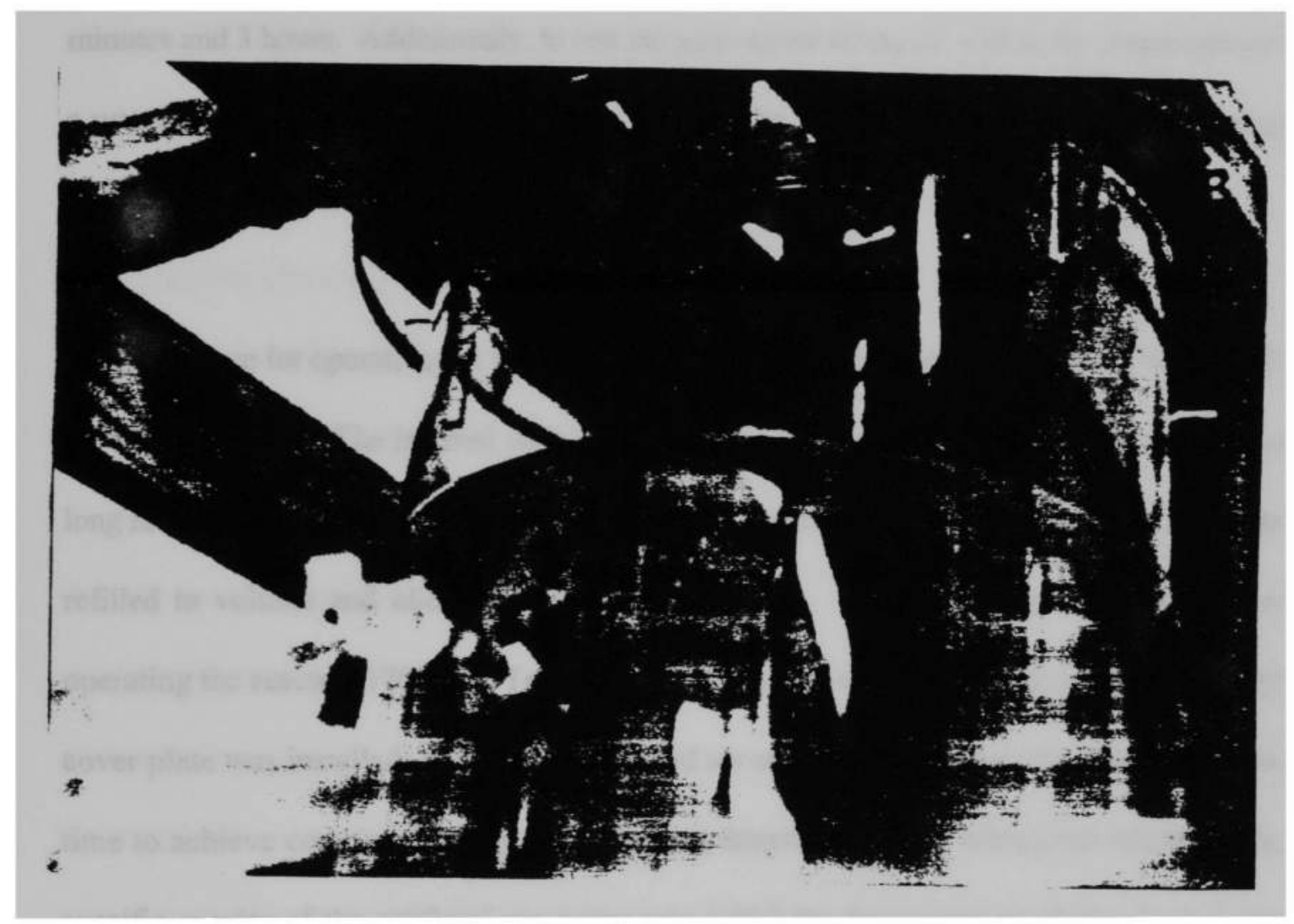

Figure 7

Adding Crude Oil to the Vertical Turbulent Dispersion Reactor 
Water samples were then analyzed to determine the chemical profile of "dispersed" hydrocarbons underneath the surface, as they relate to the physical properties of the weathered oil. Durations for simulating turbulent surface conditions were chosen to be 5 minutes and 3 hours. Additionally, to test the persistence of the oil within the water column, a water sample was also withdrawn from the reactor 3 hours after the mechanical mixing head was secured, i.e., 6 hours after initiating the experiment.

The procedure for operating the reactor begins at least 24 hours prior to the time designated for an experiment. The internal wall of the reactor was cleaned with mild dish soap and a long handled boat brush, completely filled and drained twice with clean tap water, and then refilled to volume and allowed to stabilize overnight. Approximately 2 hours prior to operating the reactor, $3700 \mathrm{~g}$ of Tropic Marin ${ }^{\circledR}$ Sea Salt was added to the reactor; the top cover plate was installed; and the mixing head set at the chosen operating speed, to allow time to achieve complete mixing and eliminate density gradients within the reactor. The specific gravity of the artificial sea water was 1.022 for the operation of the reactor. No controls were exerted on the temperature of the water within the reactor, which was allowed to equilibrate in the ambient temperature of the laboratory, and generally held fairly constant at $22{ }^{\circ} \mathrm{C}$ during the course of the experiments. 
Prior to admitting oil to the reactor, the specific gravity was recorded, along with the temperature and $\mathrm{pH}$ of the water. The $250-\mathrm{mL}$ beaker containing the $100-\mathrm{mL}$ oil sample. which was transferred out of the weathering flask (see Section 4.1 Weathering Characteristics), was weighed prior to admitting the oil to the reactor. The oil was carefully transferred to the reactor through an inlet port designed into the top cover plate using a wide throat plastic funnel, minimizing contact with the sides of the funnel. The $250-\mathrm{mL}$ beaker was then reweighed to determine the weight of oil admitted to the reactor. The quantity of oil added to the reactor was chosen to produce a standardized slick thickness of $1 \mathrm{~mm}$.

At the predesignated times, two $250-\mathrm{mL}$ water samples were withdrawn from the reactor directly into individual $250-\mathrm{mL}$ separatory funnels, from each of the designated sampling ports located at depths of 3", 27", and 63" below the water line. Equal volumes of clean seawater were transferred into the top of the reactor during the sampling process to maintain the proper water line within the reactor. The water samples containing dispersed crude oil were then extracted using OPTIMA grade methylene chloride following extraction protocols previously referred to in Section 4.1 Weathering Characteristics.

The crude oil extracts from the aqueous phase were characterized in the following fashion: 
4.2.1 Total Oil Concentration The total oil concentration contained within each extract was determined by the use of a Mettler AE 240 Analytical Balance. A 5-mL aliquot was withdrawn from one of the $11-\mathrm{mL}$ samples obtained from the extraction procedure conducted for the $250-\mathrm{mL}$ aqueous samples withdrawn from the reactor. The $5-\mathrm{mL}$ aliquot was placed into a clean disposable glass sample vial, which was preweighed (including screw cap) to determine initial tare weight. The screw cap was used as a means of labelling the sample vial for later reference. After completing the transfer of all the available $5-\mathrm{mL}$ aliquots, the vials were placed under a laboratory fume hood for a period of 36 hours to evaporate all the solvent; caps were removed and placed along-side each respective vial. When the samples were evaporated to dryness, the caps were returned to the vials, and then suitably reweighed. The differential weight was then recorded, and converted into a projection of total milligrams of oil estimated to be contained in a liter of water. Care was exercised to properly wipe down each glass sample vial with a lint-free Kim-Wipe prior to weighing. Accuracy of the Mettler AE 240 Analytical Balance was determined to be $\pm 0.00005 \mathrm{~g}$.

\subsubsection{Spectrofluorometric determination of Total Aromatic Hydrocarbon}

Concentration The total aromatic hydrocarbon concentration contained within each extract was determined by the use of a Gilford Fluoro IV Spectrofluorometer. A high performance spectrofluorometer capable of measuring in the wavelength range of $200-800 \mathrm{~nm}$ was used to quantify the aromatic fraction of the total hydrocarbons extracted from the reactor. The light source is from a high-pressure Xenon lamp, which emits a wide spectral wavelength 
range. The light beam emitted from the source is focused through a collimating lense. and is subsequently split into two beams by the excitation monochromator. One beam. the reference beam ( $10 \%$ of the beam energy), passes directly to the photo-diode detector. The second beam, the sample beam ( $90 \%$ of the beam energy), is directed to the excitation grating where the beam is focused to the selected bandwidth and then exits the monochromator exit slit. The sample beam is then directed to the quartz cuvette, which induces the sample to emit fluorescence energy in all directions. The emission monochromator, which is mounted 90 degrees off the sample beam path, to reduce interference from light scattering off the sample and the cuvette, measures the fluorescence emitted from the sample. The emission monochromator focuses the beam energy and refines the emitted light to the selected emission wavelength and bandwidth before allowing the beam to impact the photo-diode detector. A microprocessor then converts the analog data from the photo-diode to a digital display of relative fluorescence units.

The operating parameters selected for the operation of the spectrofluorometer were as follows:

$$
\begin{array}{ll}
\text { Excitation wavelength } & =310 \mathrm{~nm} \\
\text { Emission wavelength } & =360 \mathrm{~nm} \\
\text { Scan width } & =50 \mathrm{~nm} \\
\text { Scan speed } & =100 \mathrm{~nm} / \text { minute }
\end{array}
$$

A calibration curve for total aromatics was produced from 400ppb, 200ppb, 80ppb, 20ppb, 2ppb, solutions of chrysene, plus a blank solution of OPTIMA grade methylene chloride as 
a control. The fluorescence output from the calibration standards were plotted to ensure that a linear relationship was produced for concentration versus relative fluorescence (i.e., in satisfaction of Beer's Law). A linear regression analysis was performed on the output data to develop the equation necessary for the calculation of aromatic hydrocarbon concentrations from relative fluorescence data. The correlation of the data, for each of the equations developed during this analysis, were all in excess of $98 \%$ (i.e., $r^{2}$ value $>0.980$ ).

A large proportion of the original samples extracted from the reactor contained significant quantities of oil, and therefore displayed significant coloration. The coloration of the samples had an adverse effect on the performance of the instrument, as evident by an indication of zero relative fluorescence for samples which were predicted to contain significant quantities of aromatic hydrocarbons (e.g., unweathered oil, and after a 4-hour weathering period). The color either diffuses the light intensity sufficiently to limit the fluorescence response from the sample, or a compound associated with coloration was adsorbing light at or near the 310 $\mathrm{nm}$ excitation wavelength. A 10 -fold dilution of the sample, giving a zero indication previously, would subsequently give a response well beyond the calibration range for the unit as defined by the calibration standards. The dilution factors for the samples ranged from 10 1000 , and were accomplished sequentially using a $10 \mathrm{~mL}$ volummetric flask. 


\subsection{Dispersed Particle Profile}

A Coulter Doppler Electrophoretic Light Scattering Analyzer (DELSA 440) was used to investigate the distribution of electrophoretic mobility and zeta potential for particles and colloids within the range of 0.02 - 30 microns in diameter, using laser Doppler velocimetry.

Similar to the working principle of speed tracking devices used by law enforcement, the DELSA 440, analyzes particles within the sample chamber by simultaneously recording Doppler shifts in the laser light frequency experienced by 4 independent laser beams impinging on the sample from 4 different angles.

The sample cell, as shown in Figure 8, holds approximately $1 \mathrm{~mL}$ which is injected into the sample chamber by syringe, avoiding air bubbles within the cell. When an electric field is applied across the sample cell, the particles contained within the fluid will accelerate and reach maximum velocity (terminal velocity) in a matter of a few microseconds. At this maximum velocity the forces of viscous drag are in balance with the force of the electric field acting upon the particle, and the electrophoretic mobility of the particle can be recorded as follows:

$$
\mathrm{U}=\mathrm{v} / \mathrm{E}
$$

where:

$$
\begin{array}{ll}
\mathrm{U} & =\text { electrophoretic mobility (um-cm / V-s) } \\
\mathrm{v} & =\text { the terminal velocity of the particle }(\mathrm{um} / \mathrm{s}) \\
\mathrm{E} & =\text { the electric field applied the sample cell }(\mathrm{V} / \mathrm{cm})
\end{array}
$$



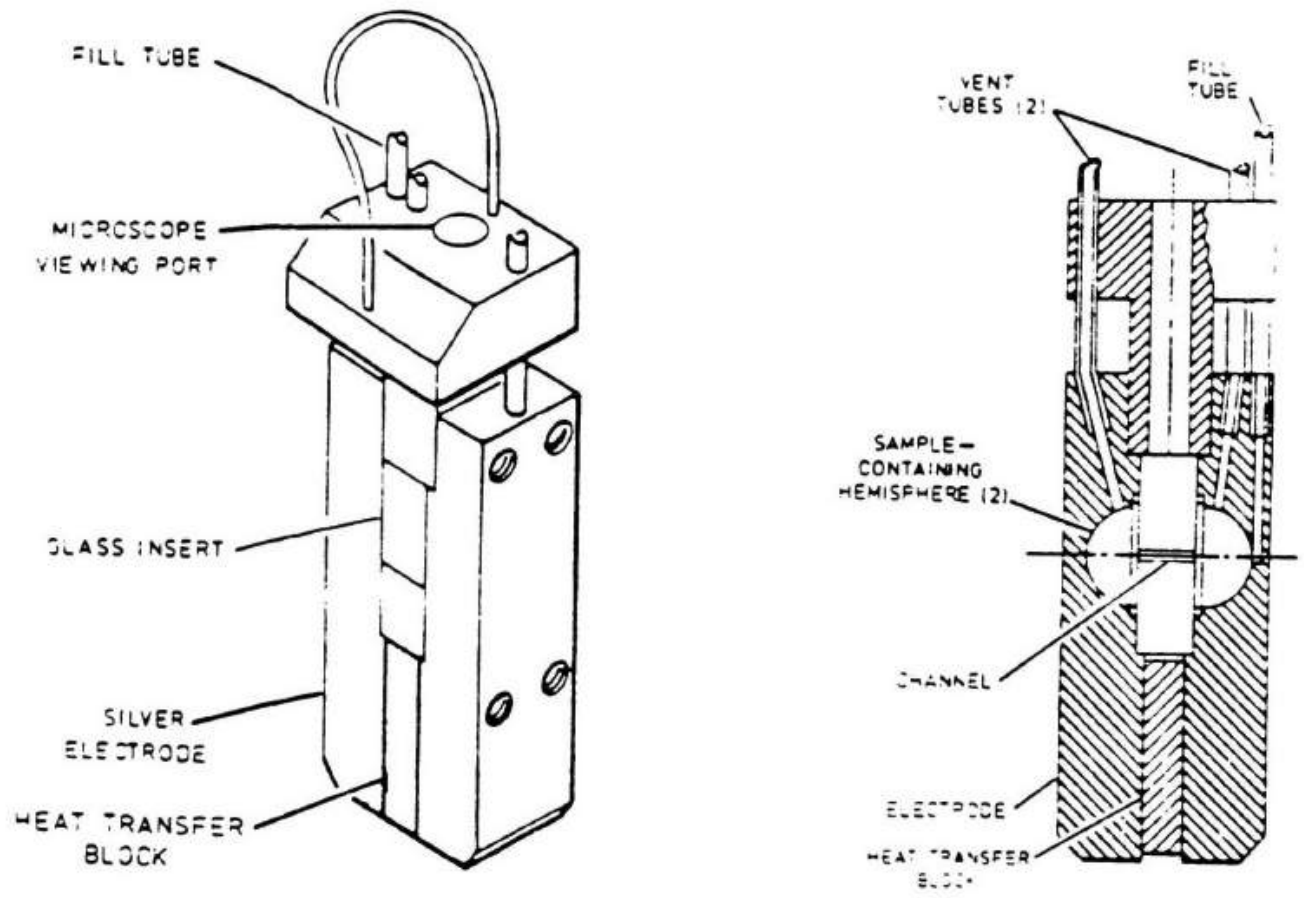

Figure 8

DELSA 440 Sample Chamber Assembly 
The zeta potential of the particle $(\zeta)$. defined as the electric potential between the bulk solution surrounding the particle and the shear plane around the particle. is related to the electrophoretic mobility by the following relationship:

$$
\zeta=4 \pi \eta U / \epsilon
$$

where:

$$
\begin{array}{ll}
\zeta & =\text { zeta potential of particle }(\mathrm{mV}) \\
\eta & =\text { viscosity of the solution ( poise }) \\
\mathrm{U} & =\text { electrophoretic mobility of particle }(\text { um-cm / V-s }) \\
\epsilon & =\text { charge of the electron }\left(4.8 \times 10^{-10} \text { statcoulombs }\right)
\end{array}
$$

In order to conduct an analysis using the DELSA 440 , the unit must first be allowed to warm up for a period of 45 minutes. During this time the sample cell was generally disassembled and carefully cleaned to remove any deposits from inside the hemispheres machined into both halves of the gold electrode, and to remove any deposits from inside the glass insert (i.e., sample channel) which might interfere with the optical pathway of the laser beams, Figure 8. After disassembling and cleaning the sample cell, the DELSA 440 requires recalibration, which consists of adjusting the cell constant of the sample cell and realigning the target point for the laser to impact the sample, according to detailed procedures outlined in the manual. 
The cell constant is adjusted by using a calibration solution of known conductivity. which is available from the manufacturer. This calibration is required because the overall conductivity of the circuit across the sample cell is a combination of the sample cell proper and the sample solution held within. In order for the unit to determine the conductivity of the solution it must have an accurate determination of the conductivity of the sample cell so that this figure can be backed out of the overall conductivity of the electric circuit. The conductivity of the solution is an important figure to obtain because it will determine the applied field strength across the cell in order to resolve the particle charges out of the information generated by the laser.

The second step of the calibration procedure is required in order to align the target point of the laser in relation to the sample cell channel width, Figure 9. The overall mobility of the particles across the channel under the influence of the applied field is a resultant combination of electrophoresis, the motion of the particles due to the field strength; and electro-osmotic effects. The electro-osmotic effects are the result of an uneven distribution of ions across the sample cell channel width due to the fact that the glass walls are at a different electric potential than the bulk solution.

This uneven distribution of charged ions sets up a parabolic flow pattern across the sample cell channel width; the flow being generated by the motion of the particles and fluid drag. In order to accurately determine the electrophoretic mobility of the particle, the laser must 


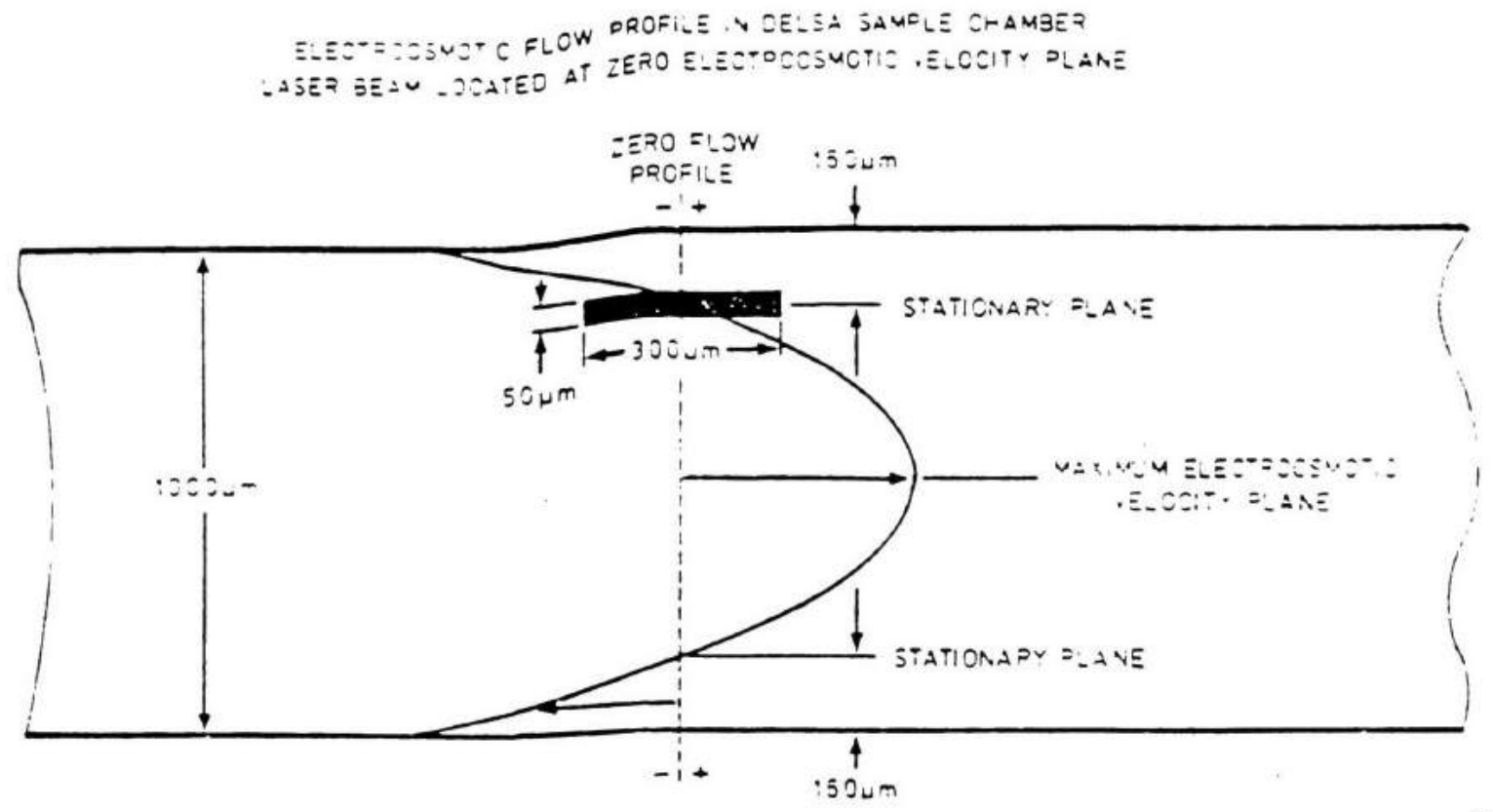

Figure 9

Location of Stationary Layer 
impact the sample at a position called the "stationary layer". This position is where the positive and negative flow directions cancel out and true mobility data on the particle can be acquired. Detailed procedures for aligning the laser target point, and the intersection point of the 4 laser beams are outlined in the operator's manual.

A confirmation of the correct positioning of the laser beam paths are obtained through the use of a mobility standard (i.e., a colloidal suspension of known size and particle mobility), which is provided by the manufacturer. The operating parameters chosen for the analysis were determined from personal site visits with the Applications Laboratory of Coulter Electronics, and listed as follows:

Particle sizing

$\begin{array}{ll}\text { Electric Field : } & 0 \mathrm{~mA} \\ \text { Run time: } & 90 \text { seconds } \\ \text { Frequency Range : } & 500 \mathrm{~Hz}\end{array}$

$\underline{\text { Particle Mobility }}$

$\begin{array}{ll}\text { Electric Field : } & 10 \mathrm{~mA} \\ \text { Field on time : } & 2.0 \text { seconds } \\ \text { Field off time : } & 0.5 \text { seconds } \\ \text { Run Time : } & 60 \text { seconds } \\ \text { Frequency Range : } & 500 \mathrm{~Hz}\end{array}$

The protocol adopted for handling the sample is one that avoids the injection of large amounts of oil to the cell which results in fouling of the cell. A $25-\mathrm{mL}$ water sample was 
extracted from the reactor along with samples extracted during tasks explained in Section 4.2 Vertical Turbulent Dispersion Characteristics and was allowed to stand for a period of 90 seconds, to reduce the amount of discrete oil droplets in the aqueous phase. The syringe tip was inserted through the slick surface and a $5-\mathrm{mL}$ water sample was extracted. The syringe tip was suitably cleaned and the sample injected into the sample cell.

Between trials the sample contained in the cell was purged, and the cell flushed with a steady stream of 2-propanol; followed by a rinsing with a steady stream of de-ionized water; then, purged to empty all remaining fluid contents. The incoming $5-\mathrm{mL}$ sample fed to the sample cell was sufficient to flush the cell with 3 complete volumes of liquid before retaining an aliquot for analysis.

\subsection{Relative Toxicity Evaluations}

Bacterial bioluminescence assay experiments were performed on a Microbics Microtox Model 500 fluorometer. The unit is designed to detect the reduction in light levels emitted from living luminescent bacteria when confronted with toxic components present in the test sample. The reduction in light levels is expressed by the parameter gamma $(\Gamma)$, and is defined as the ratio of the light reduction during the test to that of the light which remains at time (t). The light reduction during the test period is related to two mechanisms, the toxicity of the sample, and that which is associated with the drifting light levels from the reagent alone. 
Therefore a blank sample must be run initially in order to normalize the light reduction data.

Calculations for $\Gamma$ were conducted as follows:

$$
\text { Blank ratio: } R_{t}=\left[I_{t} b\right] /\left[I_{o} b\right]
$$

where:

$$
\begin{aligned}
\mathrm{R}_{\mathrm{t}} & =\text { blank ratio for time }[\mathrm{t}] \\
\mathrm{I}_{\mathrm{t}} \mathrm{b} & =\text { final light reading for the corresponding blank cuvette; } \mathrm{t} \text { minutes after } \\
& \text { the transference of the toxicant } \\
\mathrm{I}_{\mathrm{o}} \mathrm{b} \quad= & \text { initial light reading for the blank cuvette immediately prior to } \\
& \text { admitting the toxicants }\left(\mathrm{t}_{\mathrm{o}}\right)
\end{aligned}
$$

The reduction in light from the test cuvette is then normalized against the blank cell response by multiplying the initial light output of each cuvette by the blank ratio $R_{1}$ determined for time $[\mathrm{t}]$. The normalized gamma effect is then calculated for each cuvette using the following equation:

$$
\Gamma_{t}=\left[R_{t} I_{o} / I_{t}\right]-1
$$

where:

$\mathrm{I}_{\mathrm{o}} \quad=$ the initial light reading for the test cuvette at time zero immediately prior to admitting the toxicants

$I_{t} \quad=$ the final light reading recorded for the cuvette at time $(t)$

$\mathrm{R}_{\mathrm{t}} \quad=$ mean blank ratio for time $(\mathrm{t})$

$\Gamma \quad=$ the gamma effect calculated for exposure time $(\mathrm{t})$ 
Samples withdrawn from the reactor, during tasks described in Section 4.2 Vertical Turbulent Dispersion Characteristics, were extracted using methylene chloride and stored with zero head space in $11-\mathrm{mL}$ glass vials in a freezer kept at $-20^{\circ} \mathrm{C}$ until analysis. A 2-mL aliquot was withdrawn from each vial and transferred to a clean $11-\mathrm{mL}$ glass vial and brought to complete dryness under a steady stream of nitrogen. The extracts were then immediately redissolved in an equal volume of ethanol and returned to the freezer. Sample vials were sonicated under low power setting for 5 minutes prior to analysis to ensure that all the oil was resuspended in solution.

To ascertain the relative toxicity of dispersed oil as a function of the weathering period, the following modified $100 \%$ test procedure was adopted for each of the hydrocarbon extracts, after consulting with the technical staff at Microbics. A dehydrated bacterial sample was rehydrated using $1000 \mathrm{uL}$ of reconstitution solution and allowed a 15 -minute period to fully acclimate. If samples are dissolved in an organic solvent, such as ethanol, the concentration of the solvent cannot exceed $2 \%$ in any of the test cuvettes, in order to keep the toxicity associated with the solvent to an acceptable range. A 10 -uL sample was injected into the first test cuvette containing $3000-\mathrm{uL}$ of diluent, which satisfied the $2 \%$ concentration criteria. Suitable dilutions were then prepared from the original cuvette by sequential transference of a 1000-uL aliquots of diluent/sample solution between adjacent cuvettes, as per the manufactures instruction manual for the $100 \%$ test. The diluent solution was prepared from distilled water, and brought to $32 \mathrm{o} / \mathrm{oo}$ seawater using the Tropic Marin $®$ sea salt mix. The 
rehydrated mixture of bacteria was then injected into each cuvette and the initial bioluminescence recorded for time zero. The resultant bioluminescence recorded after a 5and 15 -minute periods was recorded to determine the EC- 50 value of the sample. The EC-50 value was determined by plotting the gamma values $(\Gamma)$ versus the concentration of the sample in the cuvette. The EC-50 value is actually the sample concentration which corresponds to a $50 \%$ light reduction in bacterial bioluminescence emitted from the cuvette, and is equivalent to a gamma value of $1(\Gamma=1)$. A phenol standard was prepared, as per instructions in the operators manual, and included with each Microtox analysis to check the accuracy of the procedure

\subsection{Experimental Replicability}

The analysis of crude oil weathering characteristics is highly dependent on the meteorological conditions prevailing during the course of the exposure period. The analysis conducted herein simulated the weathering of crude oil samples under natural conditions, which imposes a high degree of variability between replicate runs. This variability stems mainly from differences in solar intensity, cloud cover, localized weather patterns, and ambient air temperature. 
Table 1 delineates the recorded differences between two replicate 4-day weathering experiments. Run $\# 2$, with a higher recorded solar irradiance during the exposure period, yielded a correspondingly higher dynamic viscosity, because of the larger effects of evaporation. The effects of larger evaporative losses for Run \#2 typically results in a higher resultant concentration of natural surface-active materials present in remaining oil samples. The higher concentration of these natural surface-active materials resulted in a corresponding lower resultant oil-water interfacial surface tension, as expected. As for the resultant total oil concentrations recorded from the reactor, two comments can be put forth. First, the data coming from the vertical turbulent dispersion reactor from these two replicate runs are following the trends developed during the course of the entire research project. The higher viscosities of the oil for Run $\# 2$ results in the formation of larger oil droplets dispersed into the water column (for a fixed level of turbulence), which is the main reason why oil concentrations are lower in the reactor, especially at the bottom of the reactor. Lower oil concentrations at the surface for Run $\# 2$ can also be attributed to the early-stage formation of water-in-oil emulsions, which further restricts the vertical dispersion process. Second, the variablity of oil concentrations between samples extracted from the reactor at similar sampling sites, at virtually the same time, can be attributed to the wide scale distribution of oil droplet sizes within the reactor (i.e., from sub-micron to a few millimeters). 


\section{RUN COMPARISON}

\begin{tabular}{|l|l|l|l|}
\hline \multicolumn{1}{|c|}{ 4 DAY RUN 1 } & \multicolumn{2}{c|}{ 4 DAY RUN 2 } \\
\hline \multicolumn{1}{|c|}{ SAMPLE I.D. } & TOTAL OIL ( PPM) & SAMPLE I.D. & TOTAL OIL ( PPM ) \\
\hline 4D5M SURFACE & 1634.4 & 4D5M SURFACE & 1209.6 \\
\hline 4D5M BOTTOM & 855.0 & 4D5M BOTTOM & 621.6 \\
\hline 4D5M BOTTOM & 796.8 & 4D5M BOTTOM & 591.2 \\
\hline 4D3H SURFACE & 1930 & 4D3H SURFACE & 1061.6 \\
\hline 4D3H SURFACE & 1080 & 4D3H SURFACE & 848 \\
\hline 4D3H BOTTOM & 943.2 & 4D3H BOTTOM & 644.8 \\
\hline & & & \\
\hline VISCOSITY (cP) & 597.95 & VISCOSITY (cP) & 920.3 \\
\hline $\begin{array}{l}\text { OIL-WATER IFST } \\
\text { (dynes/cm) }\end{array}$ & 28.26 avg. & $\begin{array}{l}\text { OIL-WATER IFST } \\
\text { (dynes/cm) }\end{array}$ & 27.405 avg. \\
\hline
\end{tabular}

TABLE 1

Comparison of Replicate 4-day Weathering Experiments 
The extraction of only two or three samples from the reactor at the designated sampling time will yield a range of oil concentrations, depending on the spectrum of oil droplet sizes extracted from the reactor during sampling. Overall, the variability in concentrations in the reactor was an expected result, and since it was not that excessive, no modifications were made to the experimental design, or number of samples extracted from the reactor during each sampling.

\subsection{Ouality Control}

All protocols were implemented in accordance with best available standard methods, whose details and references are called for in this section. A concerted effort was made to assure best consistency in all activities associated with a complete experimental run. This effort covered specific aspects of sample collection, handling and analysis. For instance, all glassware was sequentially cleaned with analytical grade acetone and ACS grade methylene chloride between use to limit the potential for cross-contaminating samples. All solvents used for liquid-liquid extraction, sample dilution, and analysis by GC/MS and fluorescence spectrometry were OPTIMA grade methylene chloride only.

\section{$\underline{5.0 \text { Results and Discussion }}$}

This chapter presents and analyzes all experimental results that were collected during the weathering and dispersion phases. Efforts focus on the scientific and environmental implications of this study. 


\subsection{Physical properties}

This section describes the characteristics and values of viscosity, density, oil-water interfacial surface tension, and molecular characterization.

\section{$\underline{\text { 5.1.1 Viscosity }}$}

According to the MOBIL OIL data sheet for the Arabian Heavy Crude Oil (see Appendix A), the dynamic viscosity of the oil should be approximately $36.02 \mathrm{cP}$ at $25^{\circ} \mathrm{C}$. The oil viscosity measured 10/93 was recorded at $43.2 \mathrm{cP}$. The small difference is attributed to the handling of the oil sample and the transfer of the sample between engineering buildings on campus to access the Brookfield Viscometer.

The oil was stored in the original 1 gallon can on receipt, and during the preconstruction and materials compatibility evaluation for the acrylic reactor. The oil was subsequently transferred to nine (9) 300-mL glass containers with zero headspace 01/94, after approval of a designated set of experiments. The initial viscosity of the oil (designated as NO weathering) recorded from one of the nine (9) sealed $300-\mathrm{mL}$ bottles used during the thesis project was $118.1 \mathrm{cP}$. The discrepancy in the initial viscosity reflects the small evaporative losses, estimated at $<5 \%$, which occurred in the original 1-gallon can provided by MOBIL OIL during the 2-month storage period prior to experimentation. 
Values of oil viscosity for the weathered samples, recorded as a function of the exposure period (hours), are listed in Figure 10 (see Appendix D for data file). The viscosity of the oil was then correlated to the recorded total solar irradiance $\left(\mathrm{W} / \mathrm{m}^{2}\right)$ as shown in Figure 11, and to the mass percentage lost to evaporation during the exposure period, as shown in Figure 12 . Figure 10 shows that the viscosity of the oil increases rapidly during the first 8hours exposure, with a measured $150 \%$ increase. These results were consistent with information available from the literature. After the first 8-hours the rate of increase for the oil viscosity decreased, but remained fairly linear during the remaining 7 days, reaching an estimated maximum value of $1025 \mathrm{cP}$ at the end of the seventh day (the longest weathering period). This value was estimated from a log-log relationship developed for oil viscosity vs. total solar irradiance incurred during the exposure period, Figure 11, because of limitations of the Brookfield LVDV-II+ viscometer in measuring viscosities in this range.

The total solar irradiance recorded during the exposure period by the LI-COR pyranometer is shown in Appendix E. When these weathered oil viscosities are compared to the original properties of the crude oil delivered from MOBIL OIL, an important insight is gained for those involved in oil spill containment and clean-up. The Arabian Crude Oil (API 28) will experience a 10 -fold increase in viscosity after only 24 -hour weathering, and a 23 -fold increase after one week. This assumes that the extremely light fraction lost in the laboratory during storage, which caused the elevated initial viscosity, is rapidly lost in actual field conditions. 


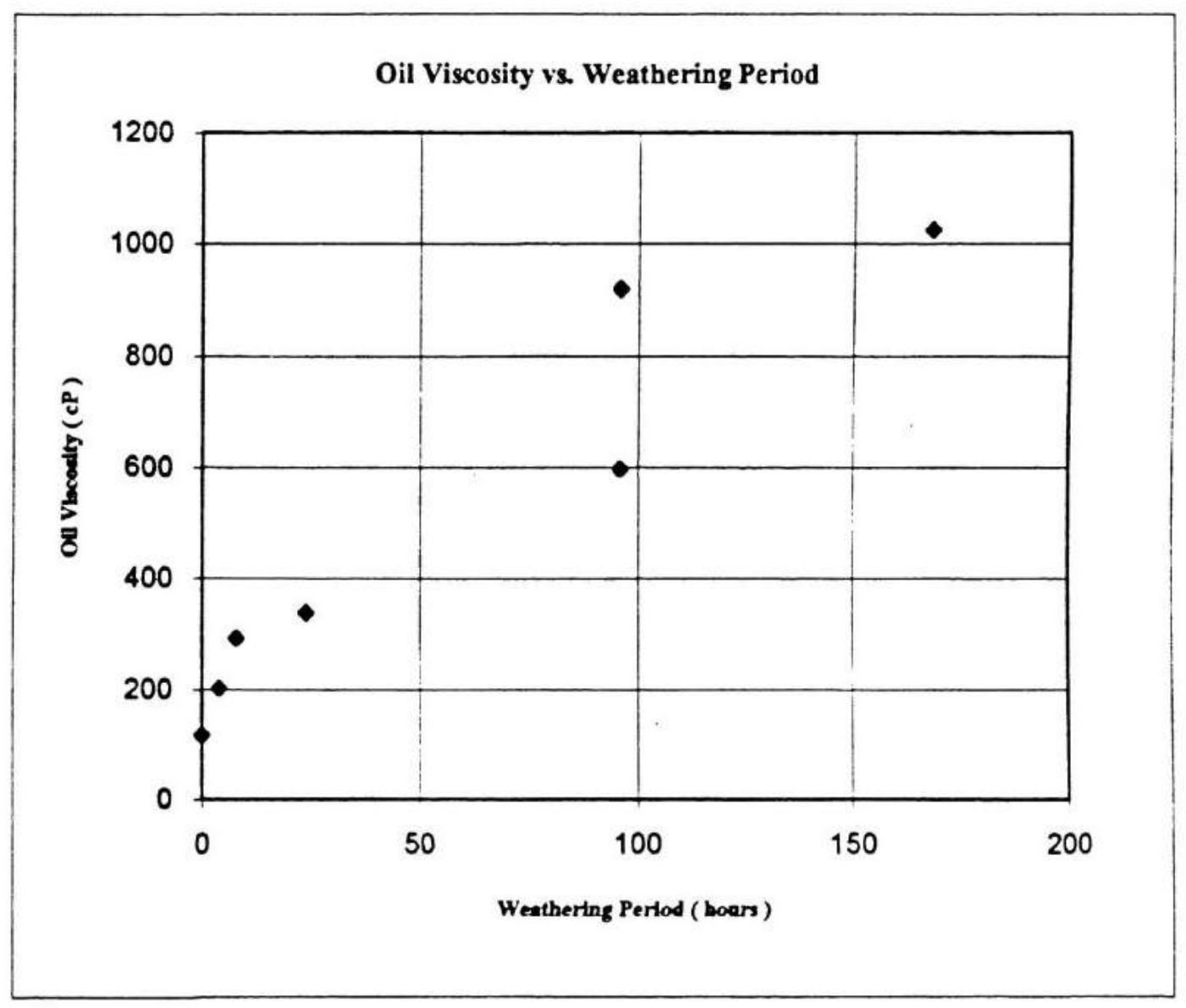

Figure 10

Dynamic Viscosity Versus Weathering Period 


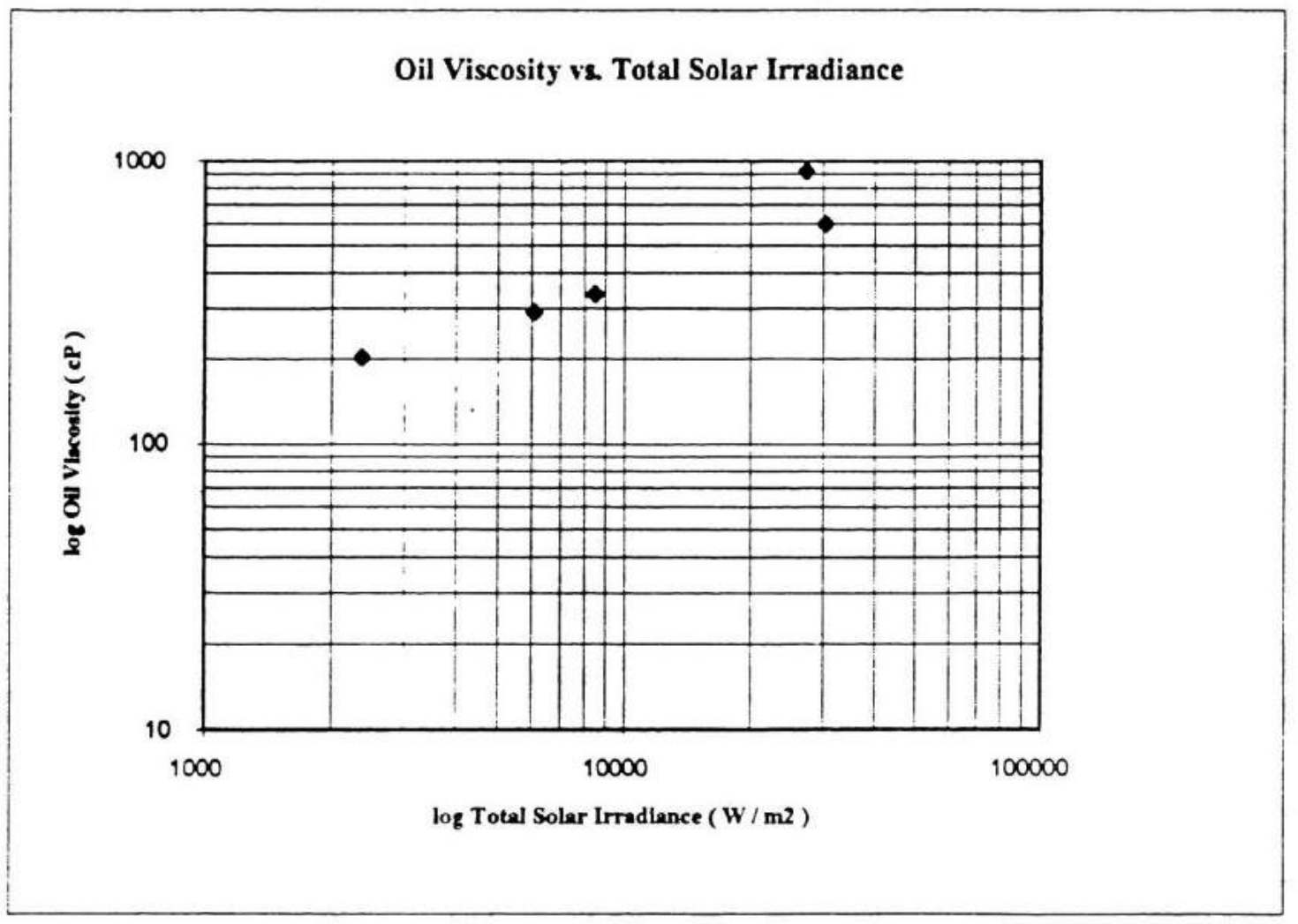

Figure 11

Dynamic Viscosity Versus Total Solar Irradiance 


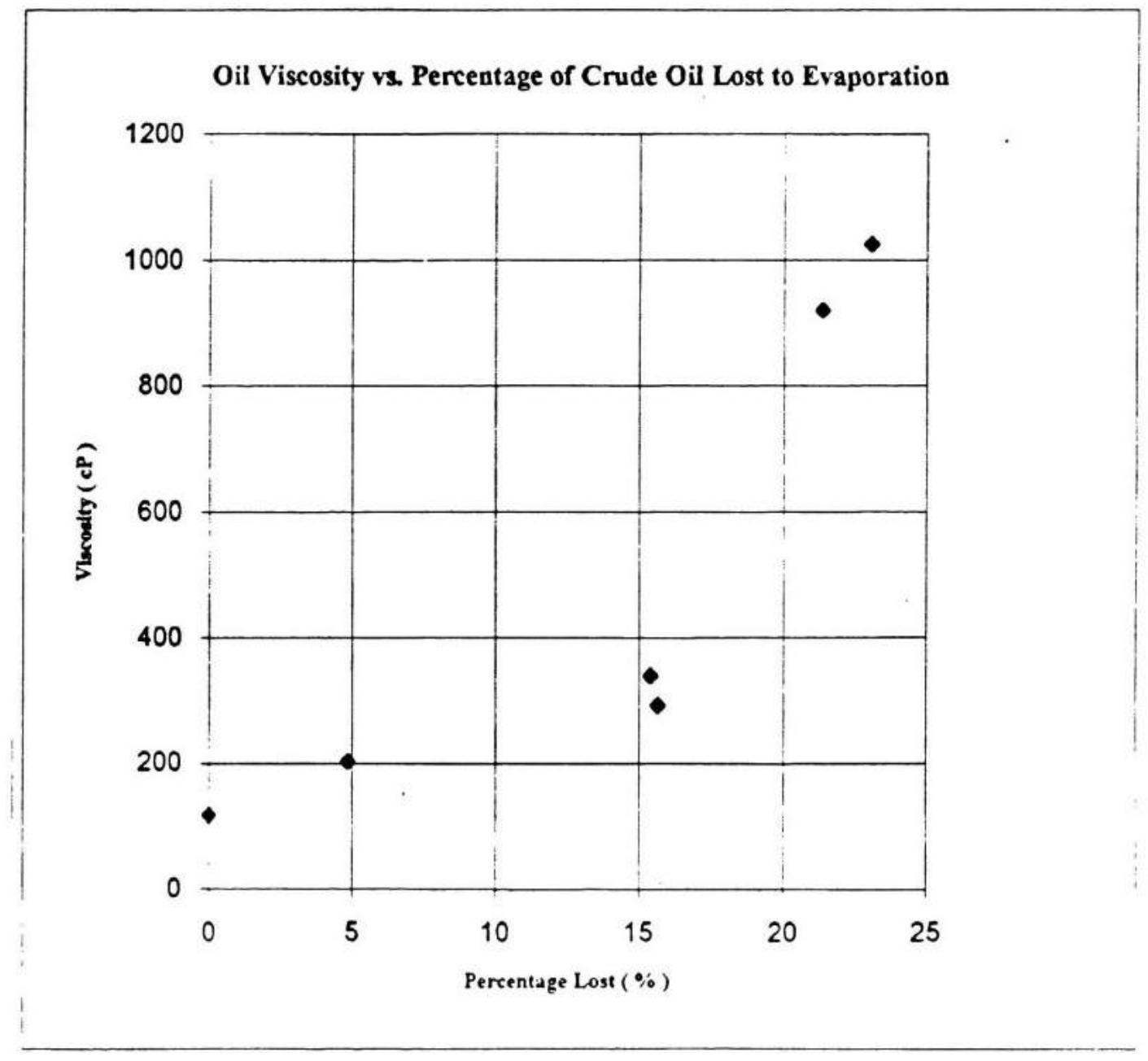

Figure 12

Dynamic Viscosity Versus Percentage of Crude Oil Mass Lost to Evaporation 
The characteristics of the oil at the end of the seventh day were drastically different from the original oil properties at the beginning of the weathering period. The oil was visually observed to be thick and viscous, sticky, with a strong odor similar to asphalt, as it would be expected at this point due to the high asphaltene concentration in the oil. The oil maintained Newtonian behavior during exposure periods up to approximately 4 days, as determined from plots of shear stress versus shear rate during viscosity determinations. The Newtonian behavior of the 4 day oil could not be confirmed because the oil viscosity approached the maximum range of the viscometer, and viscosity determinations could only be conducted at one speed ( $0.3 \mathrm{rpm}$ minimum unit speed). The 7-day weathered oil sample was more than likely non-Newtonian. This inference was based on the heavy sticky nature of the oil sample.

Changes in the physical properties of the oil during the exposure period were strongly dependent on ambient meteorological conditions, i.e., temperature, solar intensity, and wind speed. These variables influenced the micro-environment within the weathering flask, as evident by the $50 \%$ variability in the viscosity of the oil recorded at the end of two separate 4-day weathering periods (597.95 cP vs. $920.3 \mathrm{cP})$ and by the larger evaporative loss recorded from an 8-hour weathering experiment than from the 24-hour experiment.

Evaporative losses during the exposure period are depicted in Figure 13 (see Appendix F for data file). The rate of evaporation experienced during the experiments was most pronounced during the first 8 hours, with over $15 \%$ of the oil lost during this period. The evaporative 
loss during the 7-day weathering period was $23.09 \%$; less than $10 \%$ greater than that experienced in the first 8 hours. The evaporative loss experienced by the oil sample was strongly dependent on weather conditions during the experiment. The weather conditions prevailing during the 8 and 24-hour exposure periods could have not been more diametrically opposed, considering the predictability of south Florida weather patterns during the summer months. The 8-hour weathering period experienced an extreme in temperature, with $42{ }^{\circ} \mathrm{C}$ being recorded in the aqueous phase at the end of the period. The weather pattern for the 24hour period was overcast at the start, turning to intermittent rain for the remainder of the day due to unsettled weather associated with Tropical Storm Alberto, which reached as far south as Miami. The percentage of oil lost to evaporation was $15.65 \%$ during an 8 -hour period, and only $15.4 \%$ during the 24 -hour exposure period.

The evaporative loss asymptotically approached $25 \%$ towards a 7 -day exposure. Oil viscosity plotted against evaporative losses in Figure 12 indicates a steady rise during the early phase of the exposure; and, after experiencing about $15 \%$ mass loss, further evaporative losses result in increased rates of viscosity change. For example, the viscosity of the original oil was observed to rise from $118.1 \mathrm{cP}$ to $202.2 \mathrm{cP}$ after losing $<5 \%$ to evaporation, but will experience a rise in viscosity from $338.97 \mathrm{cP}$ to $1025.4 \mathrm{cP}$ in going from $15.4 \%$ to $23 \%$ total mass lost to evaporation. It is important to note that evaporative losses in the weathering flask may in fact be less than those which may be experienced in actual field conditions. 


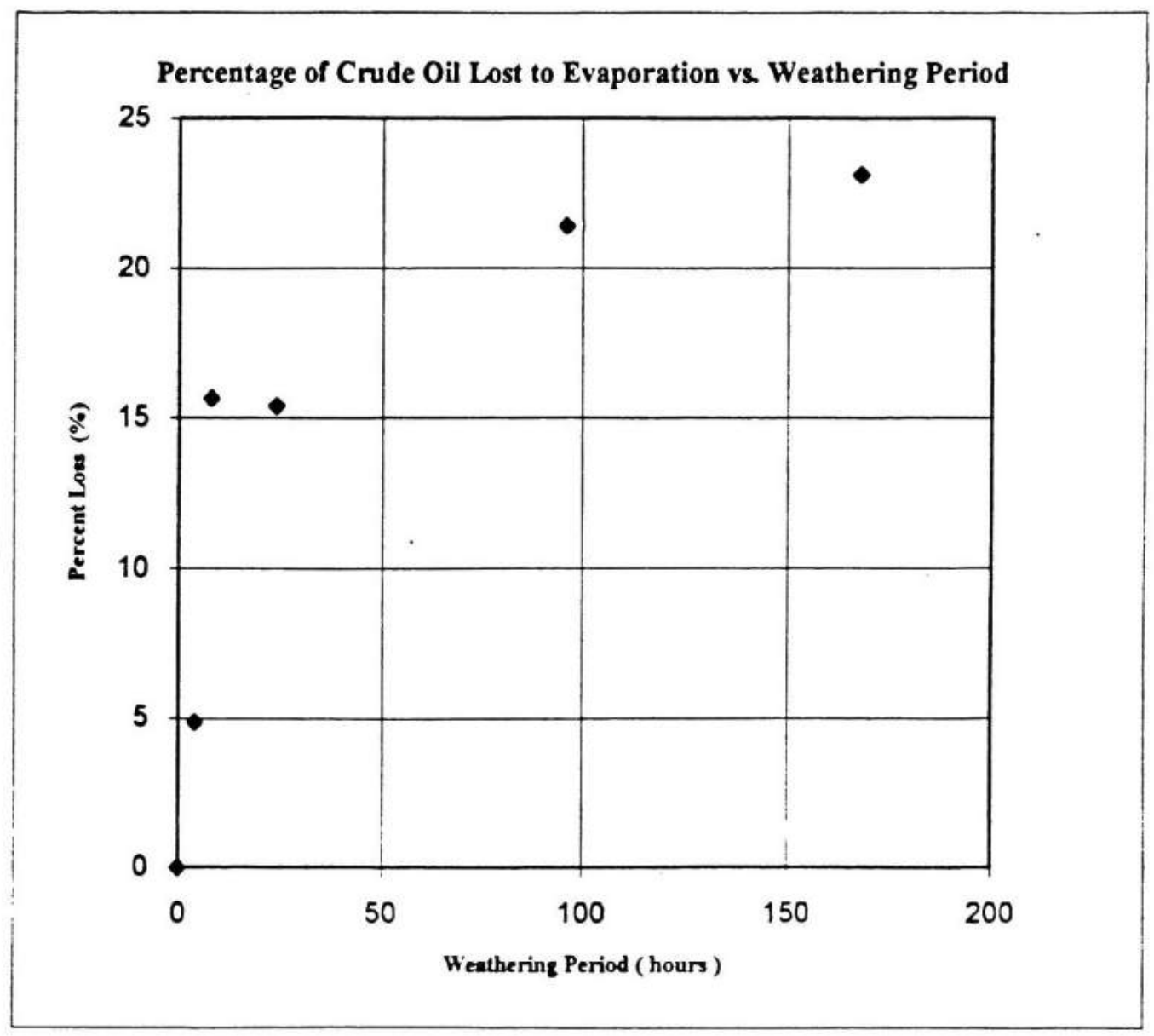

Figure 13

Percentage of Crude Oil Mass Lost to Evaporation Versus Weathering Period 
This conclusion is based on the fact that the oil in the weathering flask was obviously unable to spread out into a larger and thinner surface slick with the passage of time. The spreading of surface slicks at sea increases the mass transfer area available to other weathering processes, i.e., evaporation, dissolution, and photo-oxidation, and therefore results stated herein may be understated since evaporation rates may have been limited by molecular diffusion.

\subsubsection{Density/Specific Gravity}

The specific gravity of the fresh crude oil according to MOBIL OIL is 0.8894 (refer to Appendix A). The specific gravity of the oil prior to weathering was measured to be 0.88933. The small percentage of oil lost to evaporation while being stored in the original 1-gallon can, which caused the discrepancy in the initial viscosity, evidently had little effect on the density of the oil and is probably associated with the extremely light highly volatile fraction of the crude. The specific gravity of the oil, as shown in Figure 14, increases rapidly during the first 8 hours of exposure, which correlates with data obtained for the evaporative loses. The specific gravity of the oil asymptotically approached a value of 0.95 (refer to Appendix $\mathrm{G}$ for data file). This result is significant considering that this figure was obtained due to evaporative weathering alone. This weathered crude oil may be very susceptible to sinking if exposed to suspended particulate matter in actual field conditions prevailing along coastal environments. 

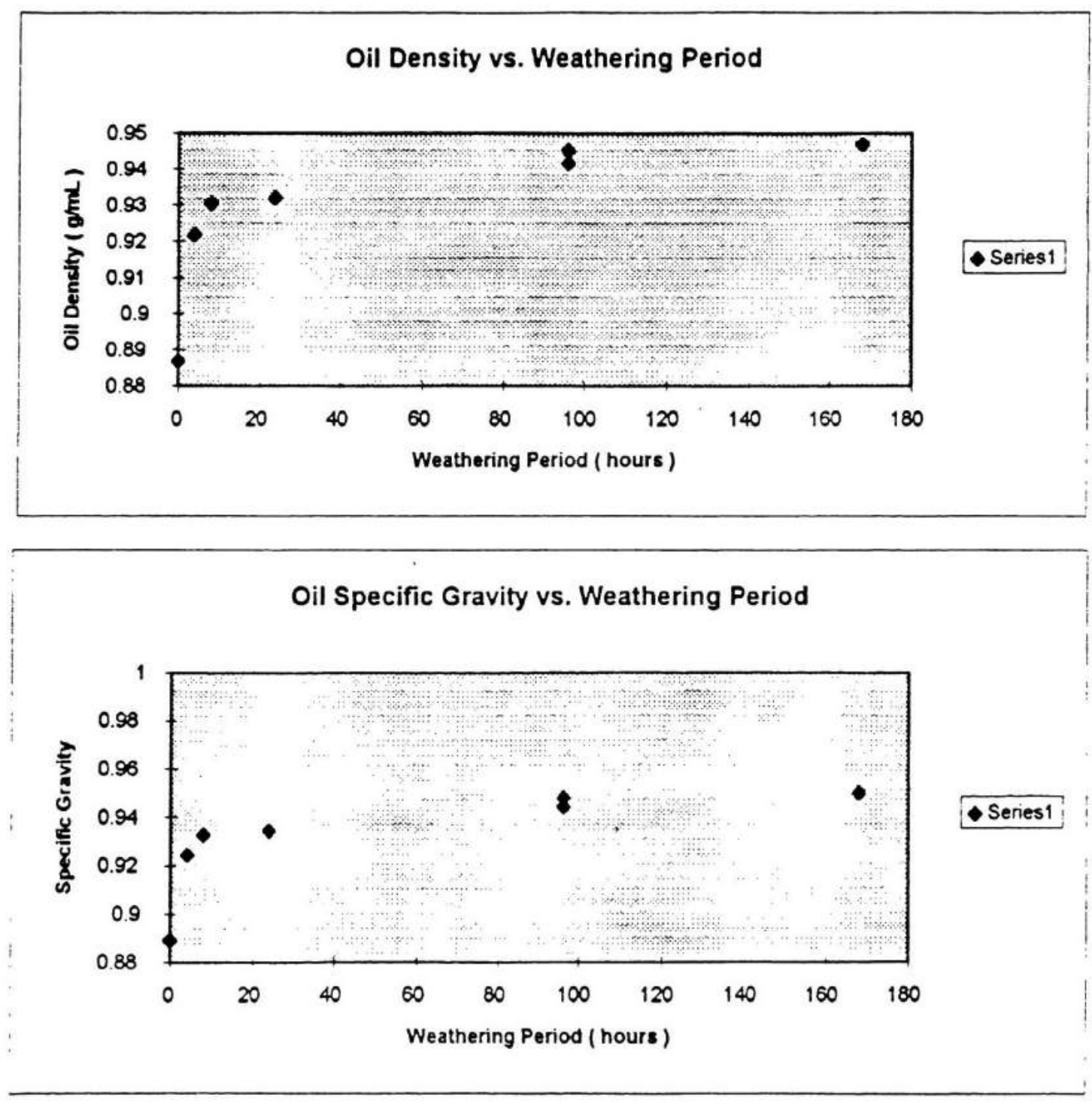

Figure 14

Oil Density and Specific Gravity Versus Weathering Period 


\subsubsection{Oil-Water Interfacial Surface Tension}

The oil water interfacial surface tension (IFST) was observed to decrease from a maximum reading of 28.4 dynes $/ \mathrm{cm}$, recorded for the unweathered oil, to a minimum of 18.6 dynes $/ \mathrm{cm}$, recorded for a 7-day weathered sample, as shown on Figure 15; see Appendix $\mathrm{H}$ for data file. The measurement of oil-water interfacial surface tension for the weathered oil samples was in each case preceded by a pre-test calibration with a known standard of $51.1 \mathrm{dynes} / \mathrm{cm}$ for hexane-water. The ratio of the actual value to the recorded value for hexane-water was defined as a calibration constant, and was applied as a multiplier to the oil-water interfacial surface tensions measured for each respective trial.

The oil-water IFST decreases rapidly and reached a localized minimum at 8 hours. The oilwater IFST rebounded slightly at the 24-hour period, but remained on a descending slope for the entire 7-day weathering period. The apparent anomaly of a localized minimum at the 8hour weathering period is probably linked to the extreme weathering conditions encountered during the exposure period. This localized minimum in the oil-water IFST provides insights into the relationship of this parameter to vertical dispersion, because the magnitude of oil dispersed into the water column reflects a localized maximum for the 8-hour weathered oil (see below). 


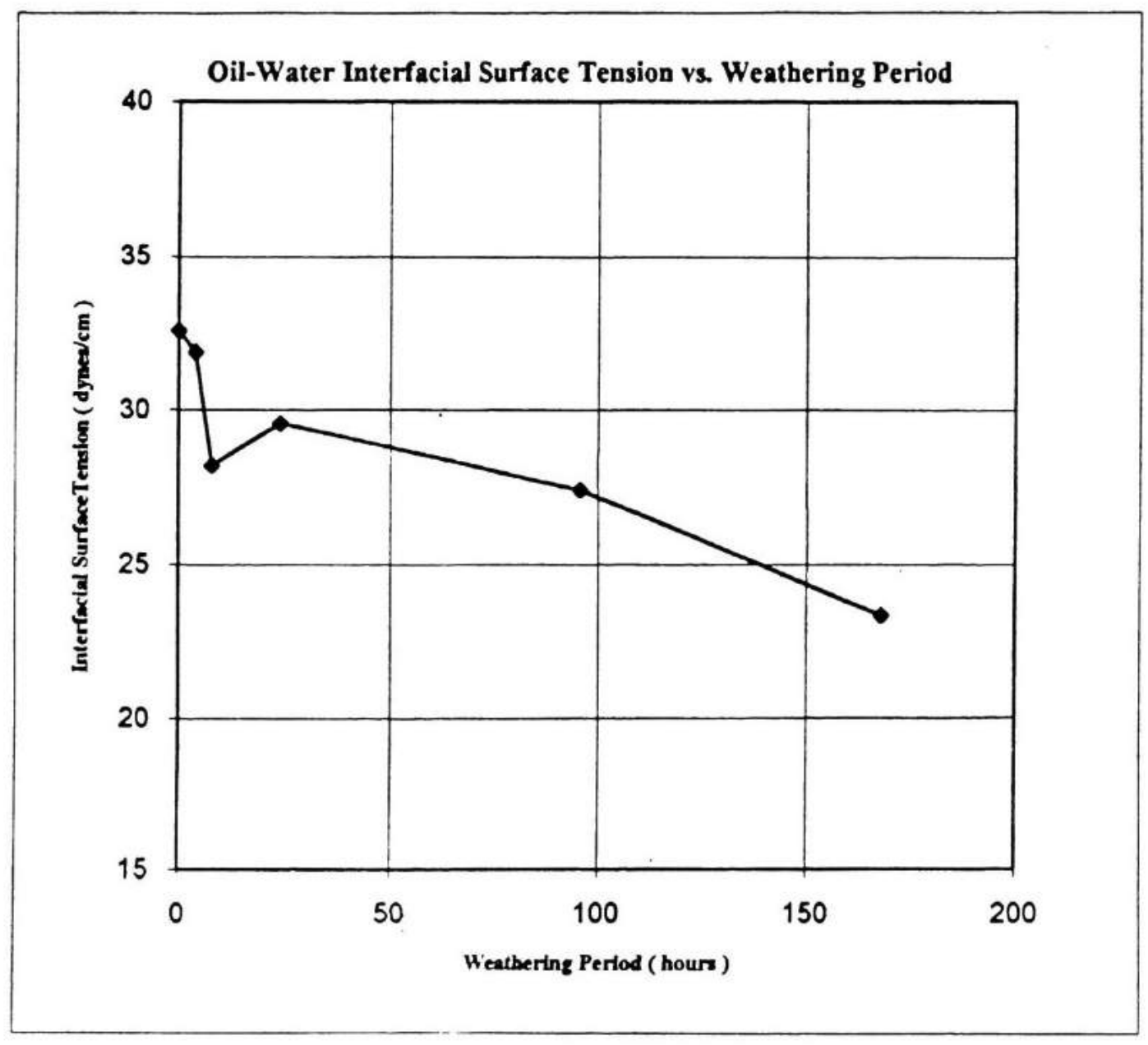

Figure 15

Crude Oil-Water Interfacial Surface Tension Versus Weathering Period 


\subsubsection{Oil Compositional Changes as Determined by GC/MS.}

The aliphatic and aromatic fractions of the weathered oil samples were characterized for the unweathered crude, along samples after $8,24,96$, and 168 hours weathering. The GC/MS results are presented in Appendix I. Results include the total ion chromatogram output and selected ion chromatograms for isolating specified families of compounds, including nalkanes (ion 57), benzenes and alkylated-benzenes (ion 91, 105 and 119), naphthalene (ion 128), methyl-naphthalenes (ion 142), di-methyl-naphthalenes (ion 156), phenanthrene (ion 178), hopanes (ion 191), methyl-phenanthrenes (ion 192), and di-methyl-phenanthrenes (ion 206). Results indicate that only trace quantities of benzene, toluene, and xylene remain in the tested samples after only 24 hours of weathering. No hydrocarbon compounds below C11 (aromatic or aliphatic) are detectable in oil samples weathered for 4 days. Plots of the relative loss rates for selected aliphatic and aromatic compounds are presented in Figures 16 and 17 , respectively. Results are presented as the time dependent abundance ratio of the selected compound indicated versus the abundance of a relatively stable (i.e., non-volatile, non-photo-sensitive) n-C-30 alkane. Results show that n-C-11 is lost at a much higher rate as compared to the longer chain n-C-26. The loss rate for n-C-19 holds an intermediate position between the two as it is expected from their physical properties. Results for the aromatic compounds indicate that naphthalene, methyl naphthalene, and di-methyl naphthalene groups are lost at a much greater rate than phenanthrene and the corresponding methylated compounds. The phenanthrene groups are relatively stable during the 7-day weathering period. Naphthalene and phenanthrene, along with the methylated groups, are 
are lost at a much greater rate than phenanthrene and the corresponding methylated compounds. The phenanthrene groups are relatively stable during the 7-day weathering period. Naphthalene and phenanthrene, along with the methylated groups, are all present in the sample weathered for 7 days, and may be responsible for the persistent toxicity of crude oil spilled in coastal environments. Anderson et al. (1974) reported that bioassays with pure aromatic compounds indicated that naphthalene and akylnaphthalenes were 3 to 10 times more toxic than benzene and akylbenzenes. The compound/n-C-30 ratio also indicates that the relative abundance of naphthalene is five-times greater than that for phenanthrene in the unweathered oil sample. The presence of naphthalene progressively diminishes with weathering, while phenanthrene remained fairly persistent, and after 7 days, the relative abundance of phenanthrene exceeded that of naphthalene. The relative abundance of methyland di-methyl- naphthalenes remained consistently greater than their phenanthrene counterparts during the seven day weathering period.

Additionally, Anderson et al. (1974) worked equalization experiments with crude oil in Instant Ocean seawater, testing for water-soluble fractions and observed that bacterial contamination affected the hydrocarbon concentration in the aqueous phase. This phenomenon would explain why an aromatic compound/n-C30 ratio would elevate; $\mathrm{n}-\mathrm{C} 30$ would be preferentially degraded in the presence of bacteria, causing the ratio to increase. The interference from bacterial contamination was not observed in the weathering experiments performed herein. 


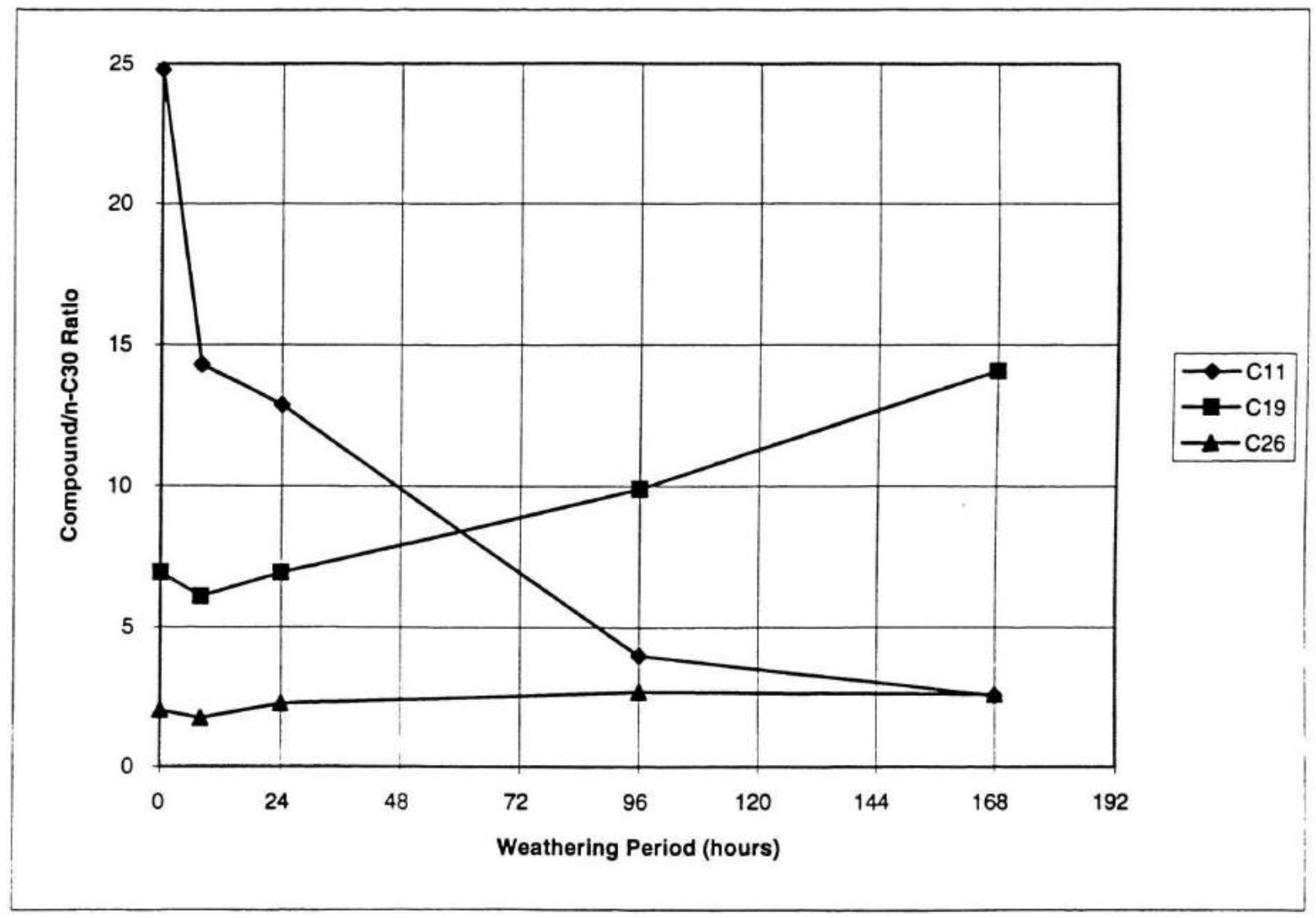

Figure 16

Relative Abundance of Selected Aliphatic Compounds Versus Weathering Period 


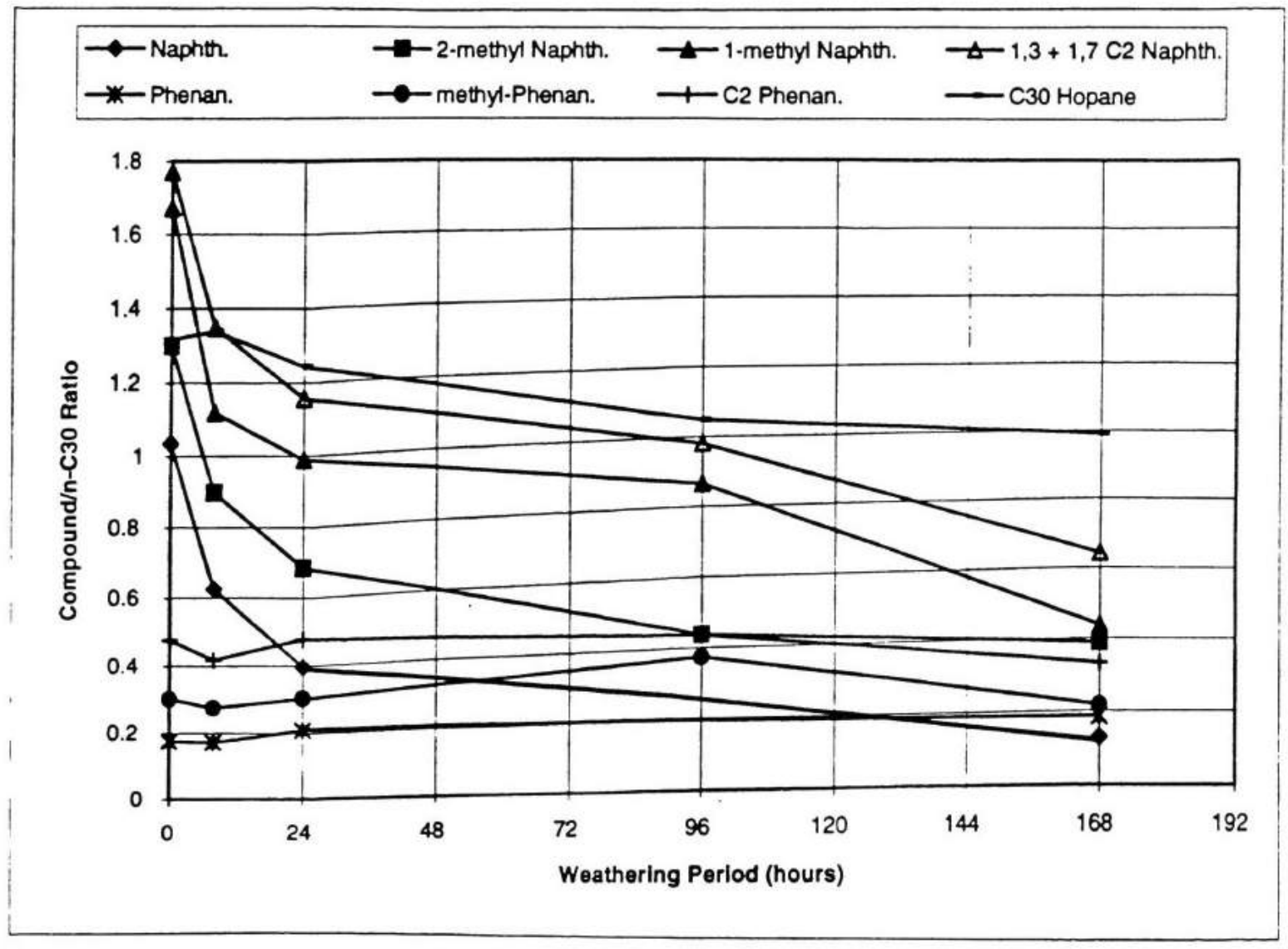

Figure 17

Relative Abundance of Selected Aromatic Compounds Versus Weathering Period 
Finally, the relatively stable and strong abundance of the hopane series of compounds, as depicted in Figure 18, and observed in this analysis, confirms the fact that this series of compounds is well suited to "fingerprinting" of oil samples in order to track an oil spill back to a possible source.

\subsection{Dispersion Characteristics}

This section presents and discusses results from the dispersion of the various weathered oils in the turbulence simulator. Data includes quantification of dispersed hydrocarbons, relative toxicity evaluations, and particle charge distributions (including average particle size) of the colloidal fraction.

\subsubsection{Aqueous Phase Oil Concentrations}

Concentrations of aromatic hydrocarbons detected in the aqueous phase of the weathering flask are depicted in Figure 19 (see Appendix $\mathrm{J}$ for data list). The concentration was observed to decrease during the first 24 hours reflecting the initial surge of soluble volatile fractions through the oil-water interface, followed by the combined effects of partitioning back through the interface and evaporation, or photo-degradation of aromatics in the aqueous phase directly. The concentration of aromatic hydrocarbons was observed to increase after the localized minimum encountered after 24-hours exposure. The concentration of soluble aromatic hydrocarbons increased steadily, to the maximum concentration recorded, after 4 days of weathering. This second pronounced peak may be the result of photo-oxidation 
File:

Operator:

Date Acquired: DON. B

Method File:

Sample Name:

Misc Info:

ALS vial:

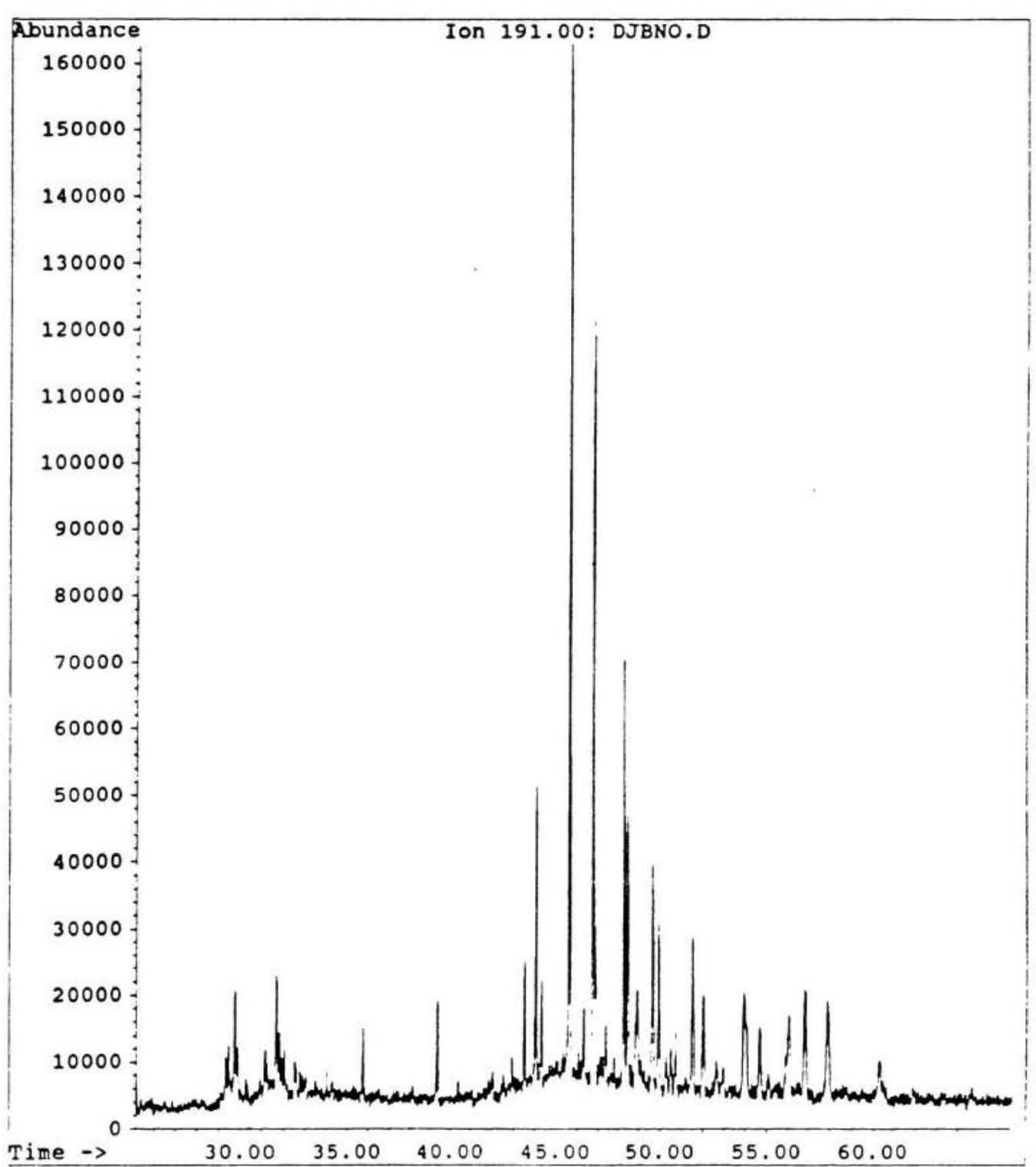

Figure 18

Gas Chromatogram for Hopane Series ( Ion 191 ) 


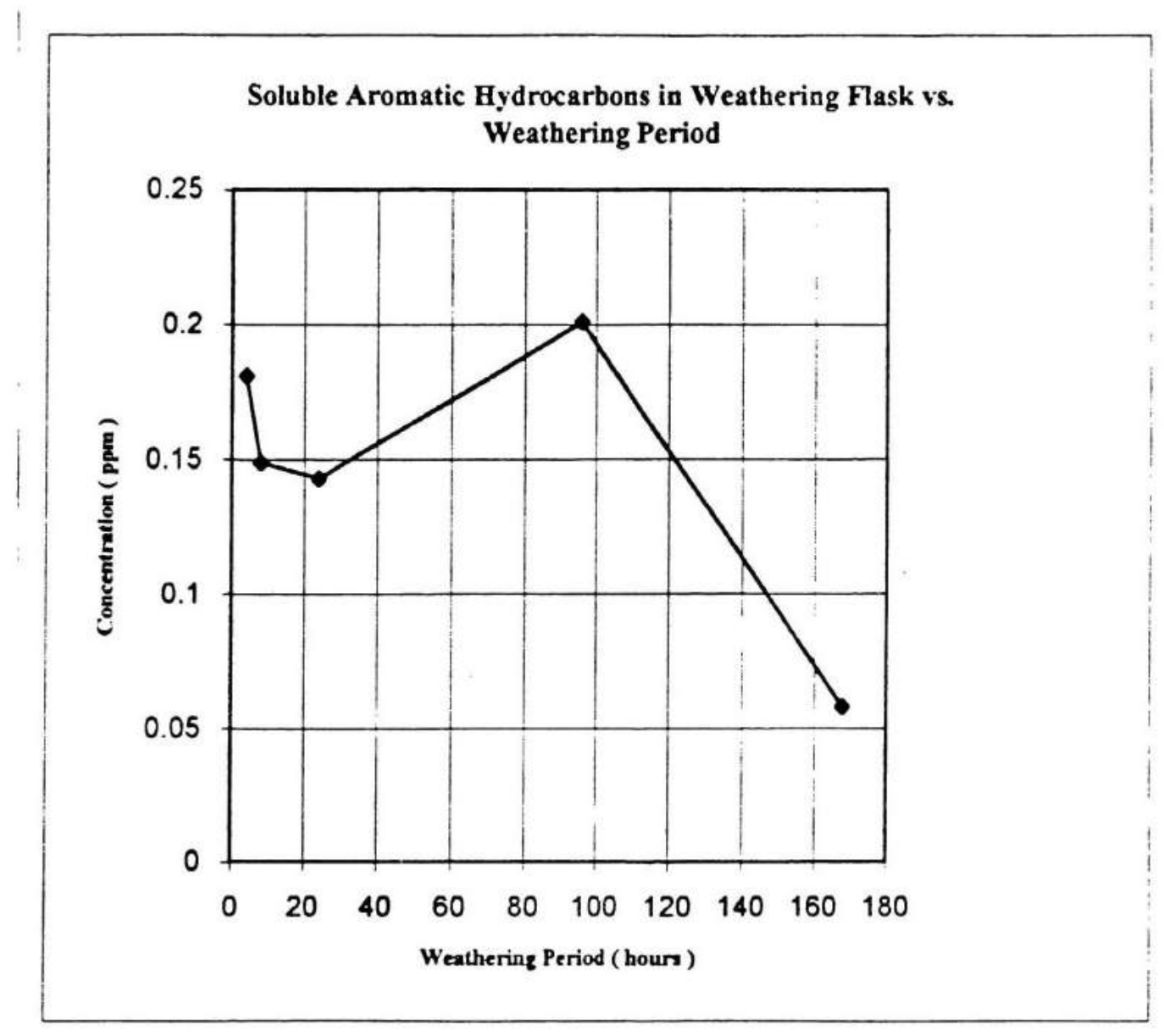

Figure 19

Soluble Aromatic Hydrocarbons in Weathering Flask Versus Weathering Period 
processes producing more soluble oxygenated intermediates. After 96 hours of exposure, the concentration of soluble aromatic hydrocarbons fell dramatically to a minimum value which was recorded after 7-day exposure. These oxygenated intermediates formed during the photo-oxidation of crude oil at sea, in addition to higher solubility (e.g., naphthalene versus naphthol), can also be more toxic to marine organisms than original parent compounds. This fact may support one possible explanation for the increased concentrations of soluble aromatic compounds observed in the aqueous phase samples extracted from the weathering flask, in association with the subsequent observation, that weathered oil samples were more toxic than the original unweathered crude, as determined by the Microtox analysis herein.

It is important to point out that the volume of oil in the weathering flask was chosen to allow for a complete physical characterization of the oil after weathering, with sufficient weathered oil remaining to produce a $1 \mathrm{~mm}$ slick in the turbulence simulator during the dispersion study. Therefore, the slick formed in the weathering flask was generally on the order of $5 / 8 "$. This may have limited the dissolution of soluble materials to the rate of molecular diffusion, especially in the extremely thick and sticky 7-day weathered oil. In actual field conditions, a surface slick acted upon by vertical dispersion mechanisms will allow for the more rapid transfer of soluble materials in the water column through the larger transfer areas associated with oil droplet formation than in quiescent laboratory conditions. 


\subsubsection{Total Dispersed Hydrocarbons in the Water Column}

The concentration of total oil within the water column of the turbulence simulator is depicted in Figures 20 through 22 (see Appendix $\mathrm{K}$ for data list). Data is configured to delineate the concentration-depth profile that exists within the reactor as a function of the degree of weathering for the oil sample. The weight of oil added to the reactor was quantified at the start of each experiment and ranged from $80 \mathrm{~g}$ to $110.2 \mathrm{~g}$ (see Appendix L). Assuming a linear distribution of oil mass, the total oil concentrations and those of the aromatic hydrocarbons were normalized by multiplying recorded oil concentrations by the ratio of the average input oil weight to the actual weight input during each trial. Normalization multipliers are also listed in Appendix L. Total oil concentrations, as expected, decreased with increasing depth during each of the individual experiments due to the oil droplet size spectrum that exists in the presence of vertically dispersed oil. The probability of encountering larger oil droplets decreases with increasing depths with fixed energy dissipation rates at the surface, due to the larger buoyant forces associated with these size droplets. At deeper depths the oil droplet size spectrum shifts to the smaller end, with lower resultant oil concentrations encountered. Additionally, the oil was observed to become more resistant to vertical dispersion mechanisms with longer weathering exposures. This effect was the result of increased viscosities of the oil with weathering.

Total oil concentrations were recorded at three depths, 3 ", 27 ", and 63 " below the surface, and at three distinct times after adding the oil to the reactor, 5 minutes and 3 hours. The 
reactor turbulence was secured after collecting the 3-hour samples. A third suite of samples was withdrawn three hours after the reactor was shut down. The total oil concentration (profiled against depth), 5 minutes after initiating turbulence, is depicted on Figure 20. The surface oil concentration ( $3 "$ depth) increased in direct relation to the weathering period, which is indicative of the larger oil viscosities encountered. Larger oil droplets with larger associated resurfacing rates and smaller entrainment depths were formed as the length of the weathering period was increased.

The sharp decrease in the oil-water IFST recorded at the 8-hour weathering period resulted in a slight localized peak in total oil concentrations at this weathering stage. The effect of the sharp reduction in the oil-water IFST after 8 hours weathering was more pronounced at the $27 "$ depth. A large peak was observed in the total oil concentration at this depth after 8 hours of weathering. The oil concentration at the 27 " depth continued to show signs of increasing slightly up to 96 hours, decreasing afterwards up through the 7-day weathering experiment. The decreasing oil concentration is a result of the shift in droplet size distributions towards larger droplets as weathering proceeds, which decreases the total oil concentration at deeper depths. The effect of this shift in droplet size distributions is more pronounced at the 63 " depth than at the other sampling depths, as indicated by the declining total oil concentrations observed as weathering proceeds. Oil concentrations obtained from the bottom of the reactor were generally on the order of one-half the concentration found at the 27 " depth. 


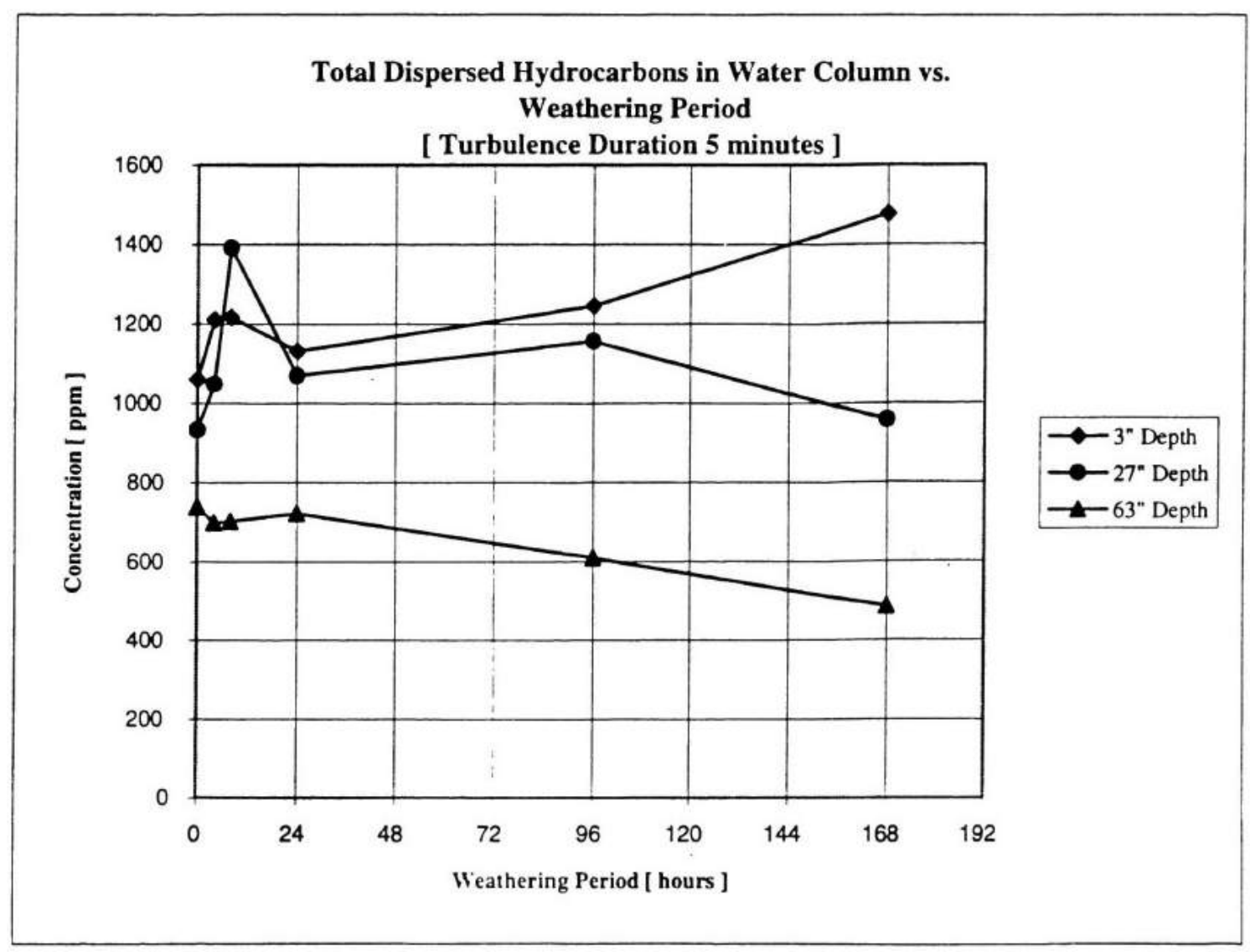

Figure 20

Total Dispersed Hydrocarbons in Water Column Versus Weathering Period [ Turbulence Duration - 5 minutes ] 
As will be shown in the following section, the general trends observed for the total oil concentrations are confirmed by the total aromatic hydrocarbons. The trends observed with total aromatic hydrocarbon concentrations are clearly defined, probably due to the higher accuracy of the analytical method.

The total oil concentration profile after 180 minutes of turbulence is shown in Figure 21. The surface oil concentration ( 3 " depth) increased rapidly to a peak for the 8-hour weathered sample. The combined effect of an associated sharp reduction in oil-water IFST and a turbulence duration of 180 minutes allowed for the production of an accentuated peak at all depths for this period. The larger peak for the 8 -hour weathered oil at 180 minutes duration, as compared to the 5 minute duration, may also be the result of the duration length. Smaller oil droplets are formed with longer turbulence periods as large droplets are subsequently sheared into two smaller droplets. This process will continue until some sort of equilibrium is attained, and a steady-state oil droplet size distribution is formed. Oil concentrations obtained from the $3 " ', 27 "$ and $63 "$ depths were more closely aligned at this sampling time, indicative of some form of pseudo-steady state distribution. At this sampling period, the total oil concentration within the reactor was observed to decrease for oil samples weathered longer than 8 hours. This effect was encountered at all depths, indicating that a reduction in available oil mass must have occurred during the period. The oil was observed to adhere to 


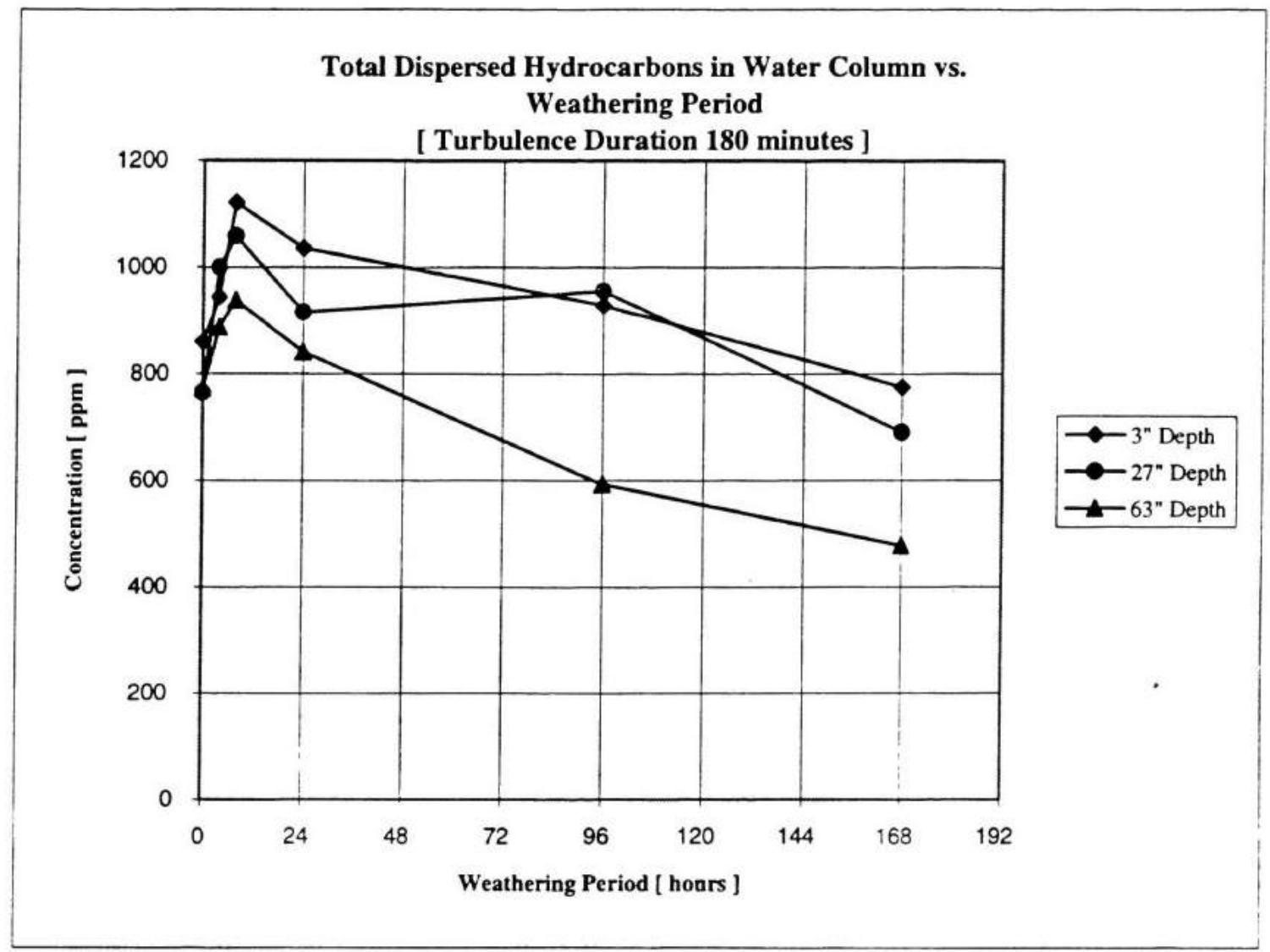

Figure 21

Total Dispersed Hydrocarbons in Water Column Versus Weathering Period [ Turbulence Duration - 180 minutes ] 
the upper parts of the reactor, including inner walls of the reactor main body and internal turbulence generator, sampling ports, propeller and shaft. This situation was most pronounced for the more heavily weathered oils (i.e., 4 days and 7 days), which were highly viscous with an associated sticky consistency and were capable of forming water-in-oil emulsions. A water-in-oil emulsion was observed to form within the reactor from a 4-day weathered oil sample, but proved to be unstable. A 50-mL sample of emulsified oil separated into a water under an oil layer overnight. The emulsion formed from the 7-day weathered oil was extremely stable, showing no visible signs of separation 2 months after originally being withdrawn from the reactor.

The total oil concentration profile measured 3 hours after the reactor was secured is shown in Figure 22. Oil concentrations measured at this time ranged between 300 and $500 \mathrm{ppm}$ depending on sampling depth and weathering period for the oil sample. The residual oil concentrations observed in the water column at the 27 " and 63 " depths at this time closely resembled the trend which was recorded for oil concentrations at 180 minutes. Oil samples from the bottom of the reactor ( $63^{\prime \prime}$ depth) were consistently higher than those obtained from the middle of the reactor ( $27 "$ depth), which indicates that some of the small oil droplets may have had larger specific gravities than that which was measured for the oil originally input to the reactor. This effect may have been the result of further dissolution of soluble lighter hydrocarbons and/or water entrainment, which would retard the resurfacing rate of the oil droplets or even promote sinking. 


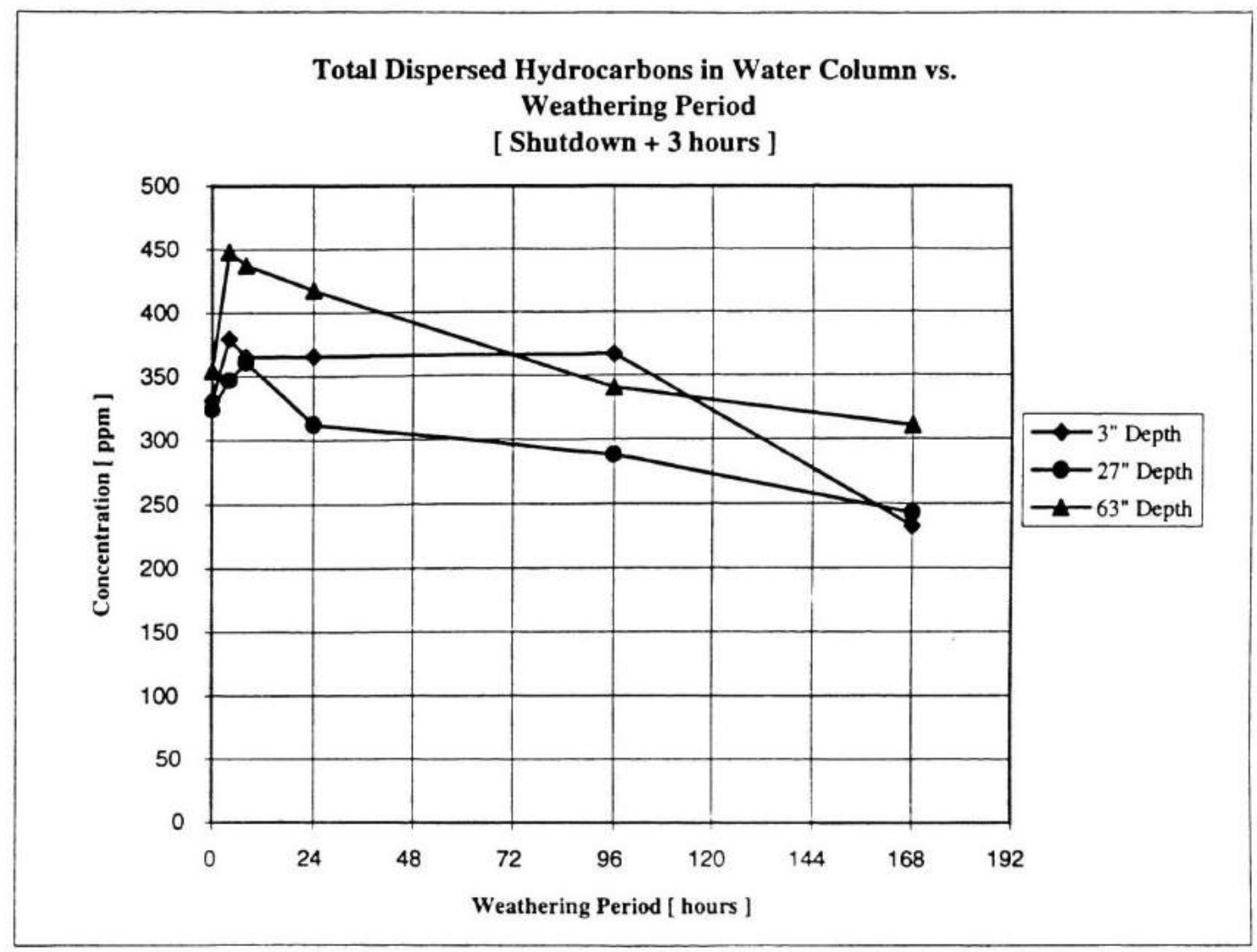

Figure 22

Total Dispersed Hydrocarbons in Water Column Versus Weathering Period [ 3 hours after turbulence generator shutdown ] 
The total oil concentration at the surface ( $3 "$ depth) remained fairly constant after the initial minor peak at the 8-hour weathering period. A dramatic decline in the surface oil concentration was encountered for the 7-day weathered oil, which resulted from the combined effects of oil adherence to internal surfaces of the reactor, especially near the surface, and the formation of a stable water-in-oil emulsion after 3 hours of mixing. The 7day weathered oil adhered to all parts of the upper works of the reactor, which may explain a lack of available oil at the surface sampling port. Additionally, oil droplets formed towards the end of the turbulence period, in the presence of water-in-oil emulsions at the surface, were actually large slugs of oil sheared off the surface mass which rapidly recoalesced with the surface slick. This skewed oil droplet distribution at the surface may have contributed to the sudden decline in residual oil at the surface. The oil droplets dispersed in the water column below the 3 " sampling port may have well been close to neutrally buoyant with extremely small resurfacing rates. Residual oil in the reactor after 7 days of weathering was also observed to have a small complement of negatively buoyant particles sinking to the bottom.

\subsubsection{Total Dispersed Aromatic Hydrocarbons in the Water Column}

Total dispersed aromatic hydrocarbons in the water column generally ranged in concentration between 500 and $900 \mathrm{ppm}$, depending on the weathering state of the input oil sample and the sampling depth, depicted in Figures 23-25 (see Appendix M for data list). Two observations were noted from the aromatic hydrocarbon data. 


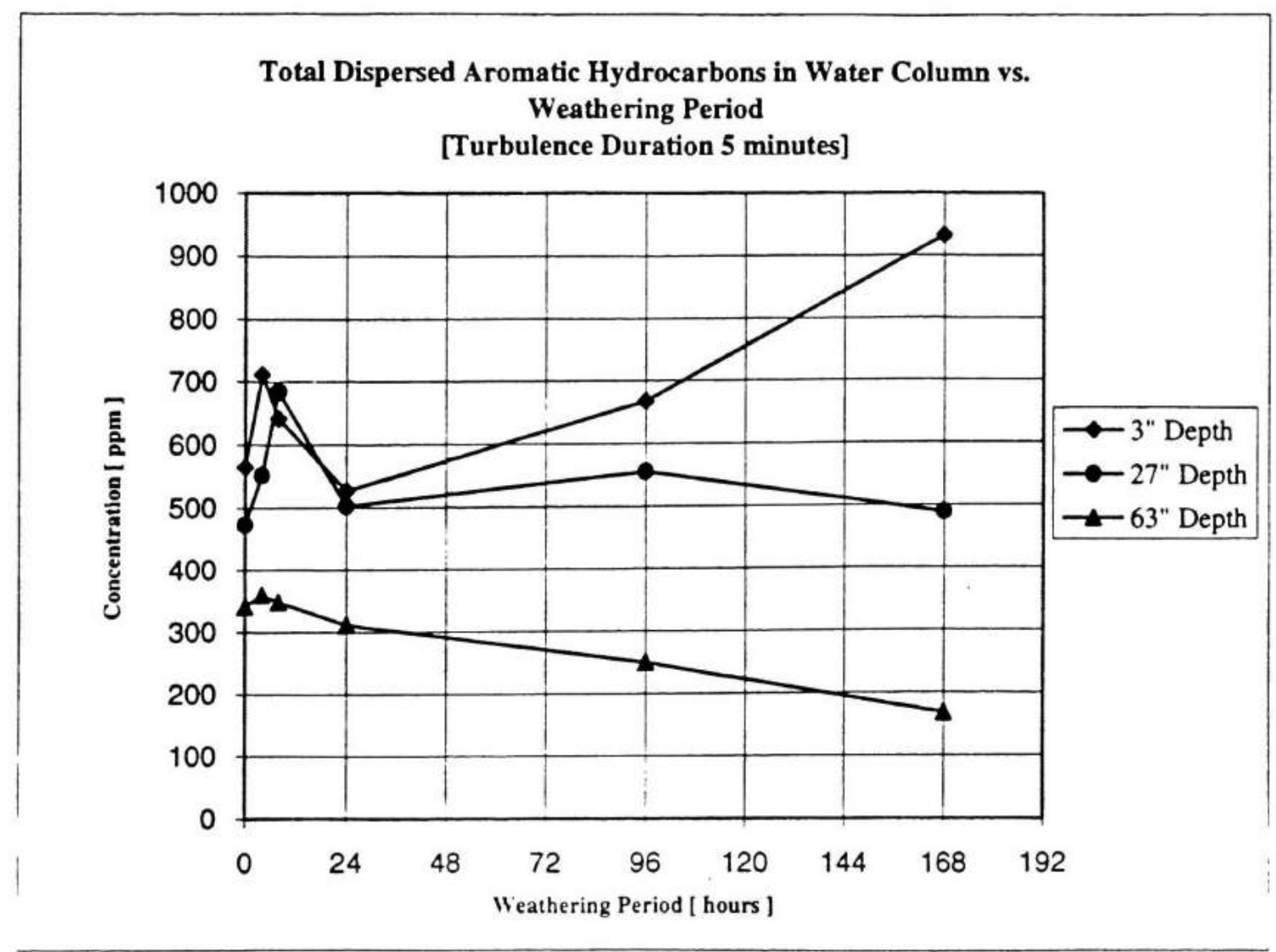

Figure 23

Total Dispersed Aromatic Hydrocarbons in Water Column

Versus Weathering Period

[ Turbulence duration 5 minutes ] 


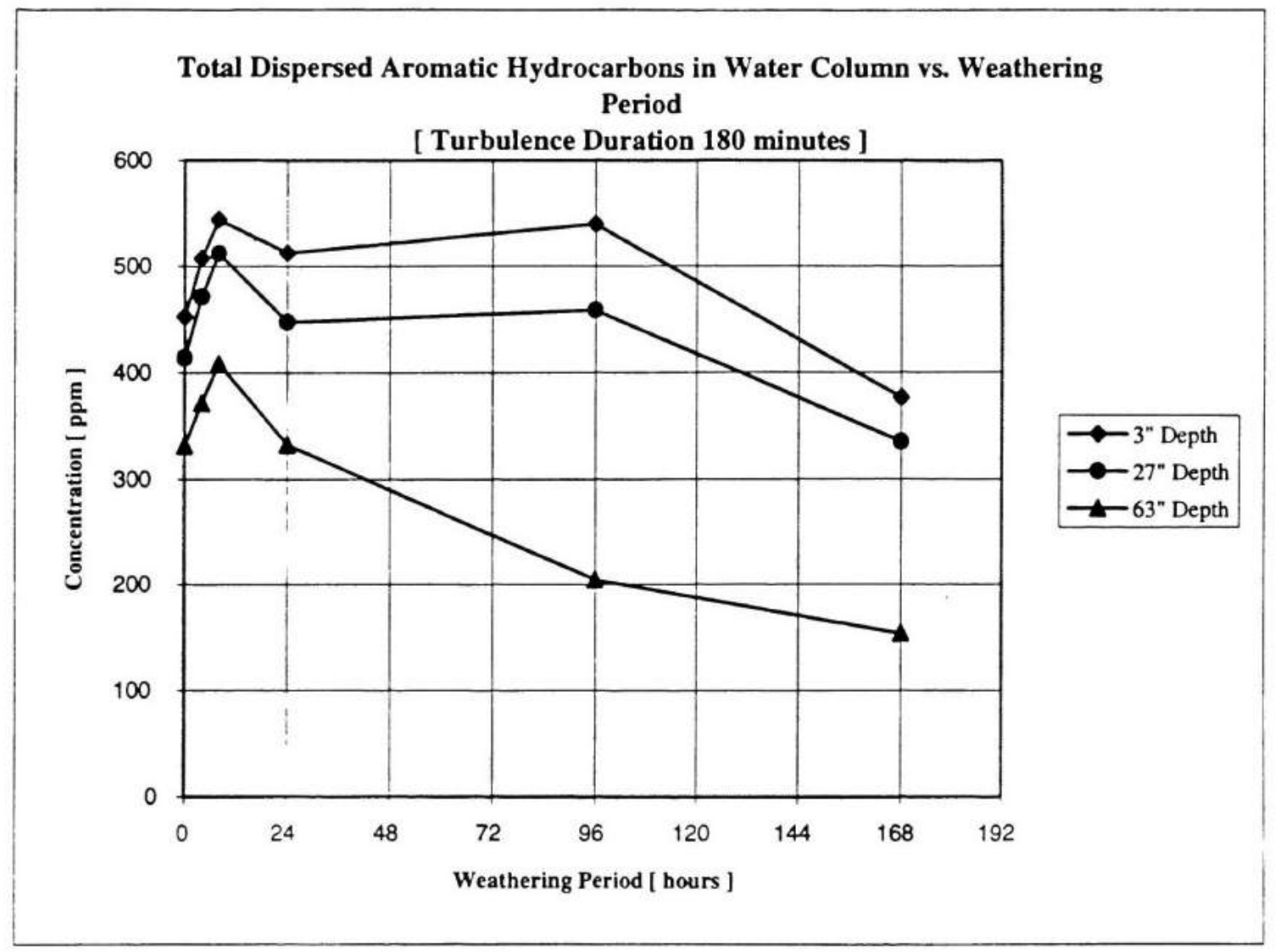

Figure 24

Total Dispersed Aromatic Hydrocarbons in Water Column

Versus Weathering Period

[ Turbulence duration 180 minutes ] 


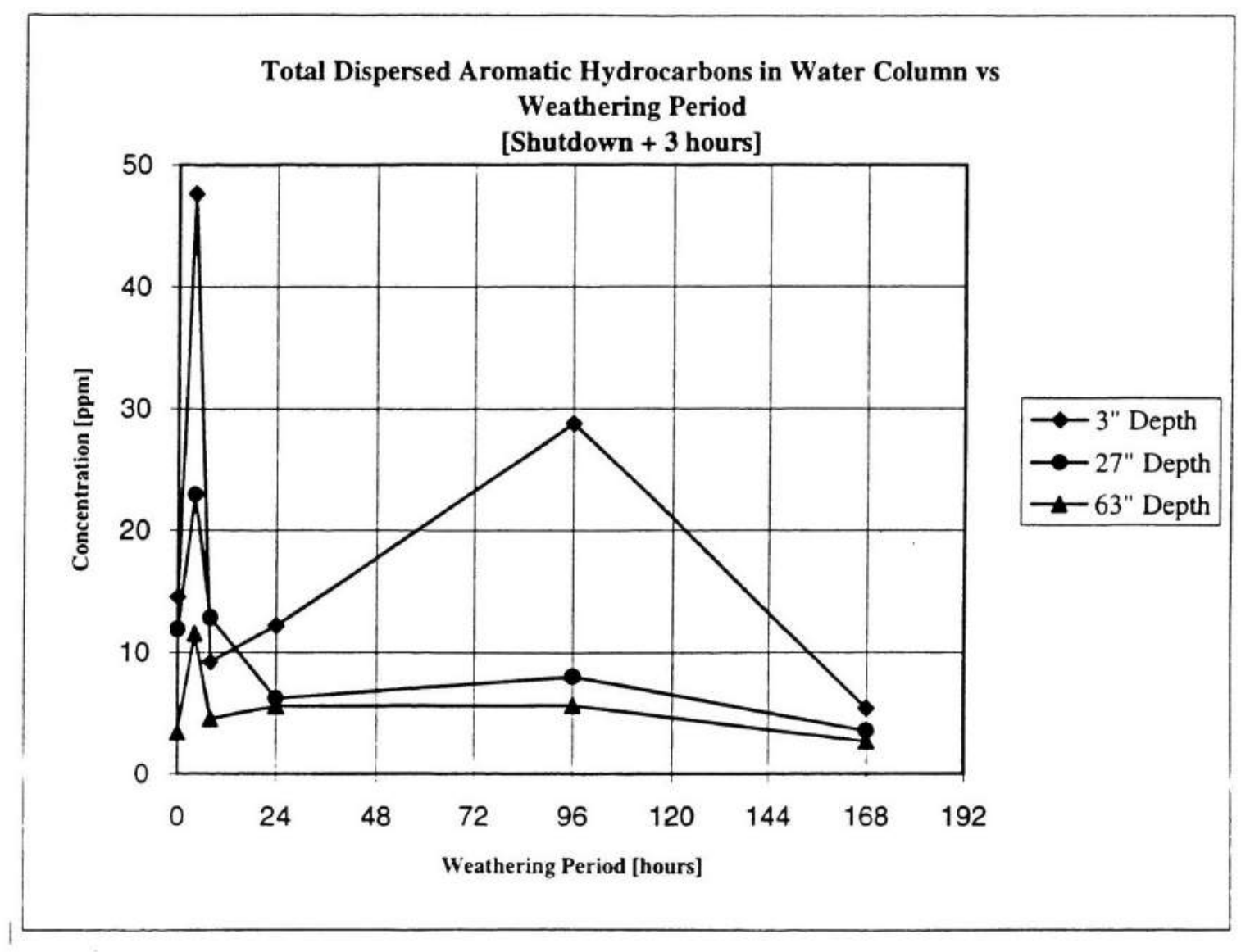

Figure 25

Total Dispersed Aromatic Hydrocarbons in Water Column

Versus Weathering Period

[ 3 hours after turbulence generator shutdown ] 
The concentration of aromatic hydrocarbons nearly mirrors the concentration patterns recorded for total oil concentration in the presence of turbulence only, with the ratio of aromatics to the total oil budget generally remaining close to the value of $50 \%$. A comparison of the unweathered oil and the 7-day oil reveals a slight increase in the percentages of aromatics as weathering proceeds, and higher aromatic percentages occurring near the surface as compared to the bottom of the reactor in both experiments. The aromatic concentrations in the water column, 3 hours after securing the turbulence, very closely resembles the aqueous phase aromatic concentrations recorded from the weathering flask, (Figure 19).

The residual aromatic concentration in the reactor achieves a maximum at all depths for the 4-hour weathered oil, possibly due to an optimum relationship between the strong presence of aromatics and the small oil droplets formed from a relatively unweathered oil; smaller droplets have longer residence times than the larger drops formed from weathered oil samples. The aromatic hydrocarbon concentration declines after this initial peak and resurges to a second peak for the 96 -hour weathered oil sample, similar to the aqueous phase concentrations previously recorded from the weathering flask. The second peak from the reactor data is not as significant as that obtained from the weathering flask, and may be attenuated due to the larger water volume to oil sample ratio found in the reactor. 


\subsubsection{Toxicity of Dispersed Hydrocarbons as Determined by Microtox}

The relative toxicity of dispersed oil droplets extracted from the bottom of the reactor, expressed as EC-50 values (ppm), are presented in Figure 26 (see Appendix $\mathrm{N}$ for data). These values represent the concentration of sample which will reduce the light emitted by a population of bioluminescent bacteria within the sample chamber by $50 \%$. As illustrated in Figure 26, the toxicity of the sample during the first 8-hours of weathering rapidly decreases, which reflects the evaporative loss of the highly toxic and highly volatile aromatic compounds, such as benzene, toluene, and xylene. After the oil weathers longer than 8 hours, it experiences a progressive shift to a more toxic configuration. This increased toxicity may be the result of the larger loss rates for aliphatic compounds compared to that of aromatic compounds, which enriches the relative concentration of aromatics in the oil sample placed in the presence of the bacteria.

Additionally, the presence of low concentration level oxygenated aromatic compounds, known to be more toxic than their parent compounds, may have formed within the slick as a result of photo-oxidation. These more toxic configurations of the original compound may have also contributed to the increasing trend in toxicity after 8 hours of weathering. The toxicity of the oil samples extracted from the bottom of the reactor reflected a higher toxicity after 7 days of weathering than that observed for the unweathered crude. Experiments conducted by Siron et al. (1991) detected an increase in the water soluble fraction along with an enrichment of polar components after two weeks of weathering. 


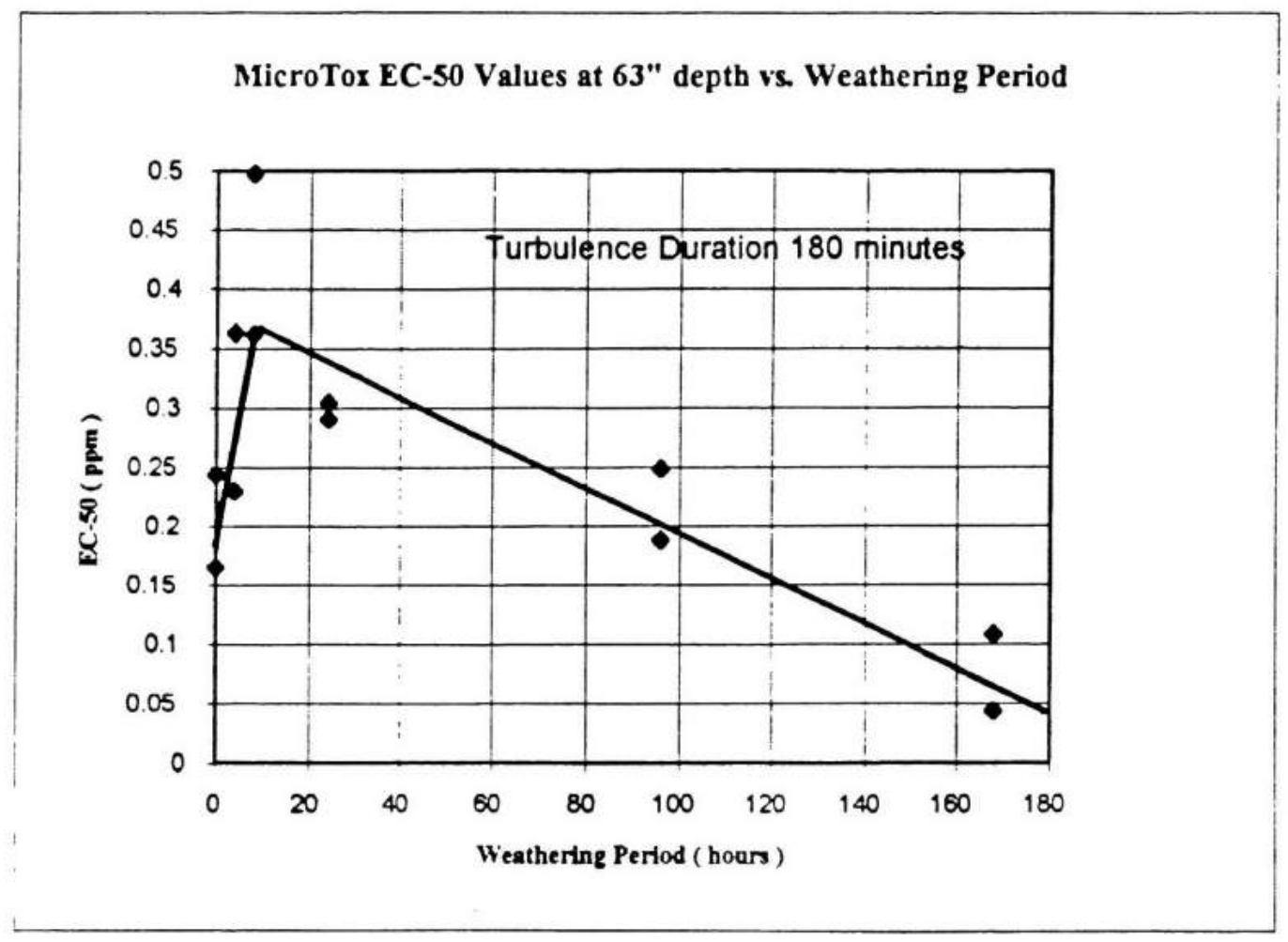

Figure 26

Microtox EC-50 Values at Bottom of Reactor Versus Weathering Period 
Siron et al. (1991) demonstrated that photo-oxidation by-products from petroleum compounds initially dissolved in the test media was occurring. These polar by-products of photo-oxidation which are known to enhance toxicity (Siron et al., 1991) may explain the greater toxicity observed from oils samples weathered beyond 24 hours in the course of this research.

\subsubsection{Particle Charge Distributions and Average Particle Size Observed in the Reactor}

The analysis of the dispersed colloidal oil fraction utilizing the DELSA 440 provided some insights, but with short-comings. The DELSA 440 is designed to investigate the charge distributions of discrete "particles" contained within a highly purified matrix. Oil droplets injected into a sample cell are not considered as discrete particles, but as one liquid medium dispersed within a larger liquid matrix. The oil injected into the sample cell resulted in a large proportion of the sample adhering to the internal surfaces of the sample cell, which progressively degraded the performance of the unit when multiple samples were being analyzed. In order to overcome this difficulty it would necessitate disassembling the sample cell and conducting a thorough cleaning, as per instructions outlined in the operator's manual. Subsequent to the disassembly of the sample cell, the DELSA 440 would require recalibration, in order to determine the conductivity of the sample cell and to reacquire the position of the stationary layer. These procedures, while necessary for the proper operation of the DELSA 440, are not consistent with the rapid sampling and analysis required when operating the vertical turbulent dispersion reactor during an experiment. To minimize the 
deleterious effects of injecting oil into the sample cell, the cell was sequentially flushed with 2-propanol and deionized water between injections.

Additionally, the artificial seawater matrix proved to be a difficult matrix to use when analyzing charged particles, especially below $2.5 \mathrm{um}$. The seawater mixture had a large conductivity, which prevented the proper confirmation of particle charge data observed by the laser unit. The seawater mixture also had a highly variable particle size in the range of $1.5-2.5 \mathrm{um}$. In accordance with the operators manual, the frequency shift observed by the unit should shift linearly with angle, in accordance with Dopplers Law. The scattering vector is proportional to the angle of incidence of the laser light on particles contained within the sample cell. Therefore, if data observed by the laser is "real", then as the incoming light angle doubles, the frequency shift associated with the particle should also double (i.e., double the angle : double the shift). A minimum shift of $10 \mathrm{~Hz}$ should also be obtained by the 8 degree beam (smallest laser angle) in order to properly resolve the data. This requirement could not be attained, given the high conductivity of the seawater matrix, even at the highest field strength setting. In order to properly resolve the particle charges out of the sample, without burning the internals of the sample cell at high field strengths, the engineers at Coulter designed a best available method for analyzing the oil dispersions, as delineated in Section 4.0 Experimental Procedures. 
Data obtained from the DELSA 440 unit is contained in Appendix $\mathrm{O}$ and is summarized on Table 2. Unstable dispersions were observed in the $1.5-2.5$ um range. Due to the highly variable nature in the droplet sizes that existed in the reactor, reproducibility in results was nearly impossible. A wide range of zeta potentials was observed to exist in this particle range. Both positive and negative charges were recorded, with recombination of oppositely charged particles being observed to occur.

Figure 27 shows the results of such recombination effects. Particle charges observed ranged from $-13.19 \mathrm{mV}$ to $+15.46 \mathrm{mV}$, with an associated average particle size of $1294.1 \mathrm{~nm}$. A second run with the same sample was run shortly after the completion of the first run, with the effects of recombination shown. The particle charge range was subsequently reduced to $-5.5 \mathrm{mV}$ to $+3.28 \mathrm{mV}$ with an associated increase in the average particle size to $1466.9 \mathrm{~nm}$. This also leads to the conclusion that the highly variable nature of the sample and seawater matrix, and the time required to prepare the sample for injection into the laser after withdrawing the sample from the reactor, proved to be highly influential on the outcome of the analysis.

Figure 28 reveals that the sample cell experiences some degree of minor fouling during the analysis of crude oil colloidal suspensions. A laser analysis using a Coulter standard with known mobility and particle size was conducted immediately after completing a suite of injections. 
DELSA 440 PARTICLE CHARGE DISTRIBUTION

AND AVERAGE PARTICLE SIZE ANALYSIS

\begin{tabular}{|l|c|c|c|}
\hline \multirow{2}{*}{$\begin{array}{l}\text { WEATHERING } \\
\text { PERIOD OF CRUDE }\end{array}$} & \multicolumn{2}{|c|}{ RANGE OF OBSERVED } & $\begin{array}{c}\text { AVERAGE PARTICLE } \\
\text { SIZE DETECTED }(\mathrm{nm})\end{array}$ \\
\cline { 2 - 4 } & $(-)$ & $(+)$ & \\
\hline NONE & ND & ND & 2249.8 \\
\hline & & & 1820.8 \\
\hline 4 HOURS & $.121-10.24$ & ND & 1917.25 \\
\hline 8 HOURS & & & 1522.7 \\
\hline & $3.22-28.25$ & $2.89-11.11$ & \\
\hline 24 HOURS & & & 1490.1 \\
\hline & ND & ND & \\
\hline 96 HOURS & & & 1758.05 \\
\hline & $3.58-36.5$ & $2.78-16.25$ & \\
\hline 168 HOURS & & & \\
\hline & $5.19-15.20$ & $.88-10.92$ & \\
\hline
\end{tabular}

Table 2

Observed Zeta Potentials and Average Particle Sizes 

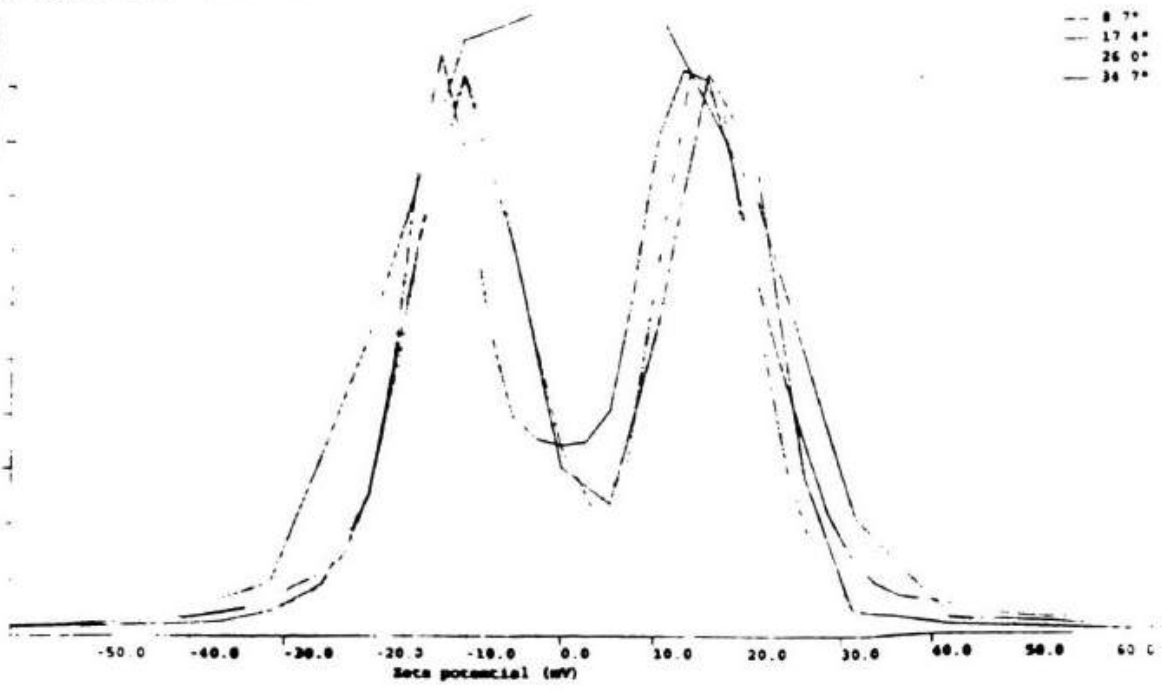

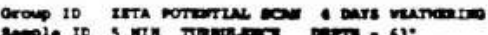

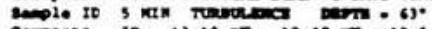

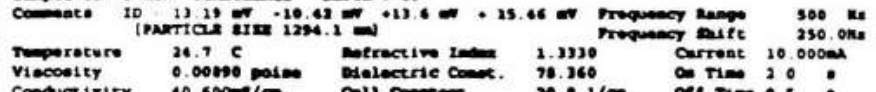

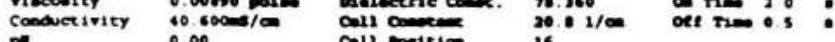

Dallo coll molition 16

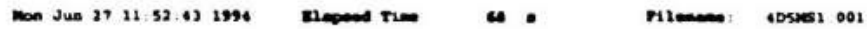

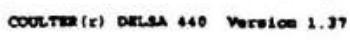

t
:
:
i
y

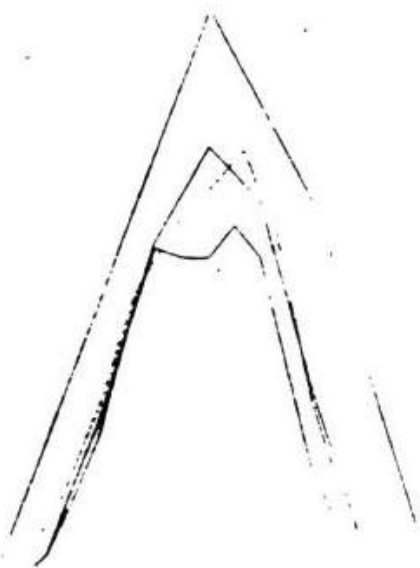

Wod 27 20:20:04 1994

$-17$.

2600
-34

- $\cdot \cdots$

$26: 0$

$-34 \%$

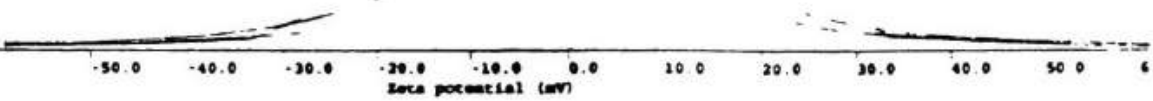

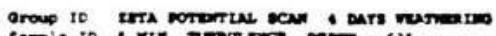

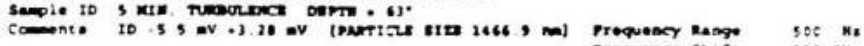

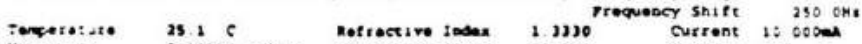

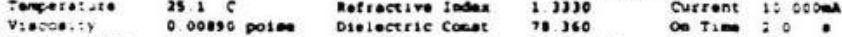

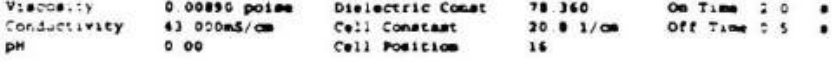

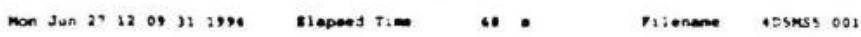

Figure 27

Particle Recombination Recorded by Coulter DELSA 440 


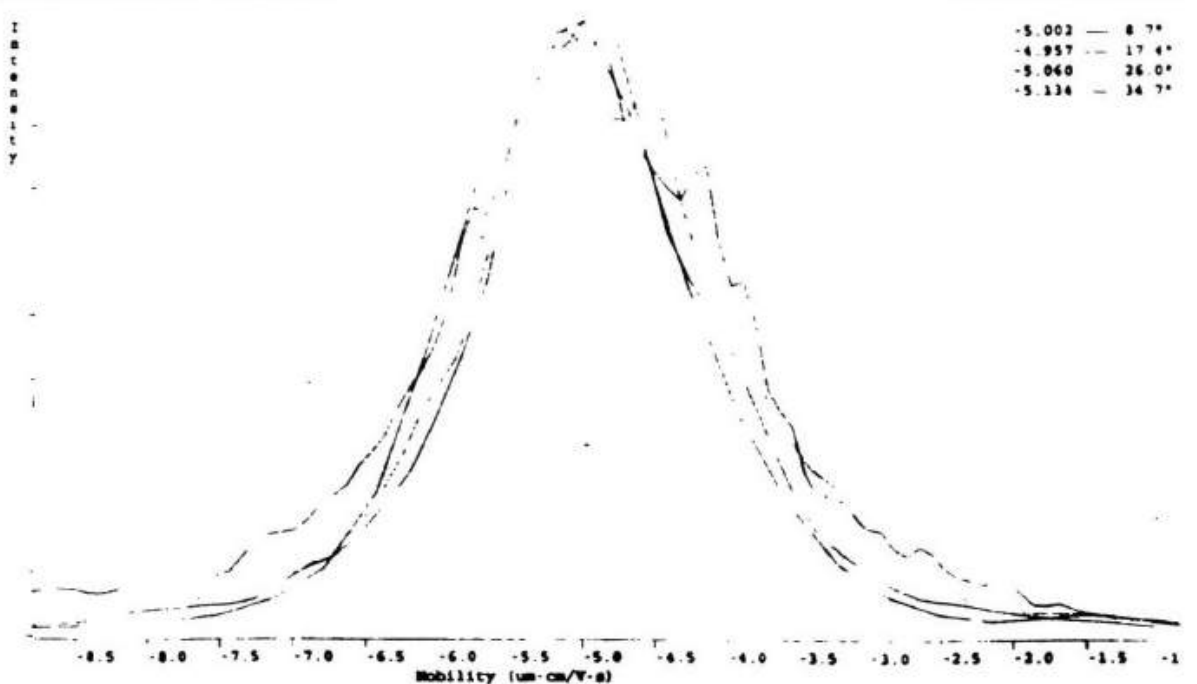

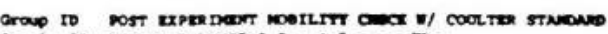

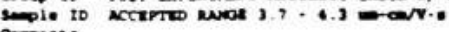

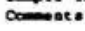

raperecure 24, 6

ricositer

condectivit

0.00
$1.23,00010$

motrace ive Indax

Desectric cont

cill comptane

und Jun $22 \quad 16 \quad 02: 21 \quad 1994$

niopees rim

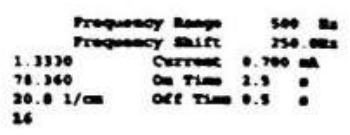

26

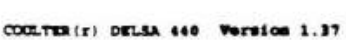

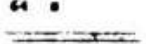

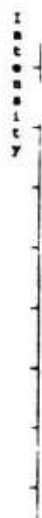

二

$-17.0$

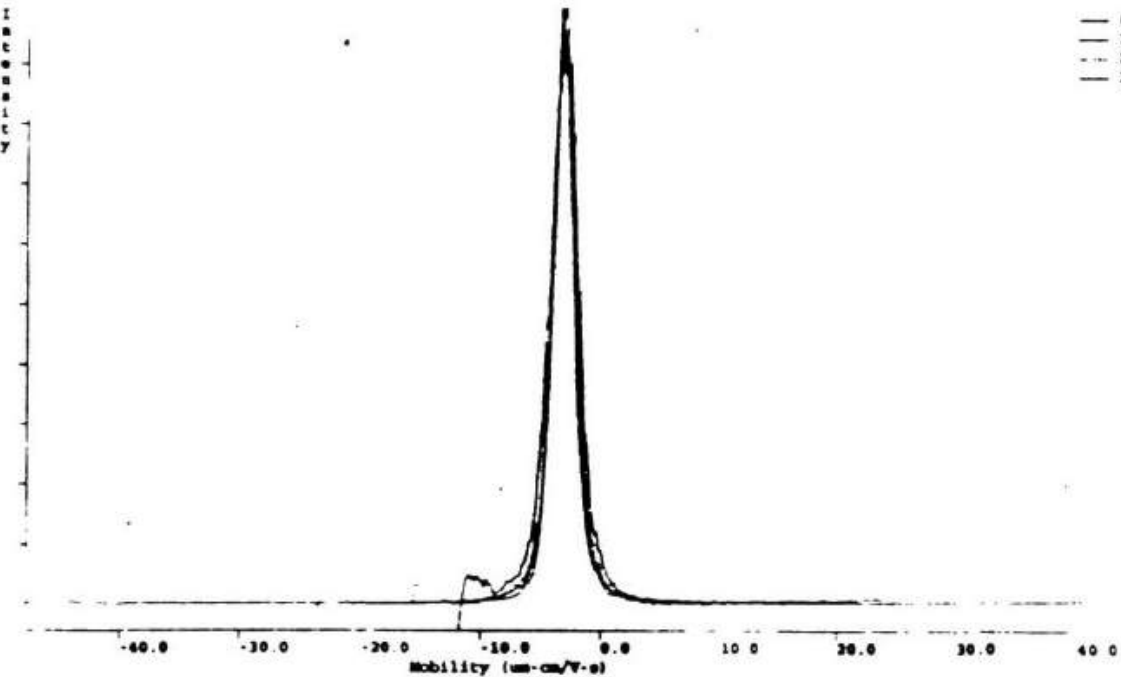

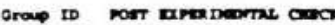

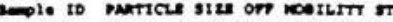

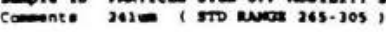

remerecture $252 \mathrm{C}$

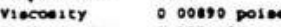

contucesivity

1. 306

notrace ive indes

Dielectrie conec.

i: corsecant

coli molition

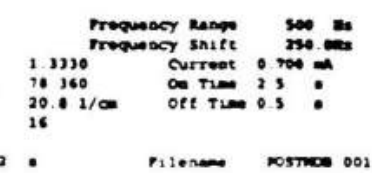

The Jun 30 16 $52: 36$ 19,

siopoes rio

72.

Pilenere nostrice 00

Figure 28

Post-Experimental Calibration Check of Coulter DELSA 440 
As indicated by Figure 28, the values obtained for particle mobility and particle size are somewhat out of tolerance, indicating that the data obtained from the oil analysis may have slightly overstated the particle mobilities, (i.e., Coulter standard being overstated in post experiment trial). This would lead to the conclusion that particle charges, both positive and negative, observed during the course of this project, while slightly overstated, in fact actually exist. The recombination effects observed in sequential trials, while identifying the presence of an unstable colloidal suspension, also confirms the existence of oppositely charged particles.

\subsection{Summary of Conclusions}

Arabian Crude Oil (API 28) was determined to weather quite rapidly, experiencing a $15 \%$ mass lost to evaporation in only 24 hours, with a corresponding 10 -fold increase in dynamic viscosity. The dynamic viscosity of the crude oil after seven days weathering was observed to increase 23-fold, which is an important consideration in terms of elevating the level of effort required for any clean-up operations.

- Weathered oil samples in the presence of simulated turbulence (i.e., energy dissipation rate approaching breaking wave conditions) were observed to form water-in-oil emulsions; albeit unstable emulsions, after a 4-day weathering period. A very stable, highly viscous, water-inoil emulsion was formed from a 7-day weathered oil sample. Effective containment measures should be well situated before any emulsification process can proceed to any great degree; for this Arabian Crude, that time-frame is approximately 4 days. 
- Density of Arabian Crude Oil after 7 days of weathering approaches a value of $0.96 \mathrm{~g} / \mathrm{mL}$. This value was attained from mainly the evaporative loss of lighter weight hydrocarbons. Sinking could be a real possibility for this oil, when in the presence of typical SPM concentrations found in the coastal zone.

- Oil-Water Interfacial Surface Tension was observed to decrease fairly uniformly during a 7-day exposure period, with a typical range of 20-30 dynes/cm. A localized minimum value was attained around 8 hours weathering, with a resultant corresponding peak in oil dispersion observed after 8 hours.

- The concentration of aromatic hydrocarbons under the slick drops during the first 24 hors of weathering. Only trace concentrations of benzene, toluene, and xylene could be detected after 4 days of weathering. Higher soluble aromatic concentrations were detected in the aqueous phase from equalization experiments between weathered oil samples and artificial seawater, possibly due to photo-oxidation processes, which form oxygenated compounds with higher solubility. Similar trends were also observed in residual aromatic hydrocarbon concentrations recorded from the vertical turbulent dispersion reactor.

- The toxicity of the weathered oil samples were determined to be more toxic to bioluminescent bacteria than unweathered oil samples, as indicated by the EC-50 values obtained from a Microtox Analyzer. No hydrocarbon compounds below C-11 can be detected after 4 days of weathering. Naphthalene, methyl- and di-methyl-naphthalenes, phenanthrene, methyl- , and di-methyl-phenanthrenes were all present in a 7-day weathered 
oil sample. These compounds, along with any corresponding oxygenated compounds, may be responsible for the persistent toxicity observed for weathered oil samples.

- Concentration of oil was observed to decrease with depth within the vertical turbulent dispersion reactor. The probability of encountering larger droplets decreases with increasing depth, as determined from relationships between oil droplet size and resurfacing rate.

- Significant dispersed oil concentrations were still detected in the vertical turbulent dispersion reactor 3 hours after surface turbulence was abated. Concentrations generally exceeded $300 \mathrm{ppm}$, which provided some insights into the persistence of oil in the water column, especially with droplets having diameters $<1 \mathrm{~mm}$.

- Unstable oil dispersions in the size range, believed to be 1.5 to 2.5 um, were observed using electrophoresis techniques. A wide range of corresponding zeta potentials were recorded, with negative charges being more prevalent.

\subsection{Recommendations}

- Further analysis of the weathered oil samples by GC/MS should be conducted in an effort to ascertain the time dependent concentrations of the aliphatic and aromatic fractions; determined on a per unit mass basis from weighed oil samples. In this capacity, time dependent changes in the compositional changes of the crude oil, as a function of the weathering period, can be accurately quantified. It is further recommended that the characterization of the crude oil also include the OXY-PAH fraction, so that the potential products of any photo-oxidative process be quantified. The compound specific concentration 
data determined from GC/MS analysis will enable better correlations to Microtox toxicity tests; identifying causal factors responsible for increased toxicity of weathered crude oil samples observed during this research effort. Toxicity evaluations, as a specific point analysis, should be conducted from the aqueous phase directly, thereby eliminating excessive sample manipulation, interference from solvent exchange and the inherent toxicity associated with the solvent directly.

- The experimental design for weathering the crude oil can be enhanced by providing a means of limiting excessive temperatures in the weathering flask; eliminating the magnetic stirring feature and immersing the weathering flask in a small pool of water may be one solution worthy of further investigation. Additionally, the inclusion of flash point to the list of crude oil physical properties analyzed, as a function of the weathering period, may be worthy of investigation; no information was found in literature, but would be of interest to those involved in emergency response to actual oil spill sites.

- The adherence of oil to the internals of the vertical turbulent dispersion simulator interfered with the collection of total oil and total aromatic hydrocarbon data after 3 hours of running the reactor; causing a drop in oil concentrations at all depths in the reactor, especially for heavily weathered oils. Once the oil is admitted to the reactor, equilibrium was rapidly attained at the level of turbulence used in this project, therefore the operational timeframe for the reactor should be drastically shortened from 3 hours used herein; approximately 30 minutes would probably be sufficient to acquire the required data. 
- The analysis of oil droplets using the Coulter DELSA 440 is difficult based on the capabilities of the unit. The analysis of colloidal particles requires a highly purified matrix, with water filtered through a 0.2 um filter recommended by Coulter in the operators manual. Oil droplet dispersions in seawater run counter to this requirement. It was also determined that Milipore filters are unsuitable, because of the presence of surfactant wetting agents, or fibrous debris from the filter, showing up in the output of the laser run in the particle size range of 2 um. Similarly, de-ionized water obtained from the Drinking Water Research Center at Florida International University contained particles present in the laser output at 2-2.5um, which are believed to be particles off the ion-exchange membrane. Prepared seawater, herein used, was determined to be undesirable from the stand-point of impurities, and the associated extremely high conductivity. Oil injected into the sample cell had the drawback of contaminating a perfectly calibrated sample cell in a short period of time. Additionally, oil adherence to the internals of the glass insert (i.e., sample chamber) interfered with data acquisition, which necessitated flushing the cell with 2-propanol, and reinjecting another sample. The wide distribution of dispersed oil droplet sizes contained within a typical sample also made reproducibility difficult. Particle sizing was highly variable and unpredictable. A Coulter counter capable of sizing the oil droplet distribution within the reactor as a function of the weathering period, may have provided the means of achieving the original objective (time-variant dispersed oil droplet sizing) and should be considered as a valuable acquisition for future studies. 


\subsection{REFERENCES}

Al-Rabeh A.H., Cekirge H.M., and Gunay N. (1989). A Stochastic Simulation Model of Oil Spill Fate and Transport. Applied Mathematical Modelling 13, 322-329.

Anderson J.W., Neff J.M., Cox B.A., Tatem H.E, and G.M. Hightower (1974). Characteristics of Dispersions and Water-Soluble Extracts of Crude and Refined Oils and Their Toxicity to Estuarine Crustaceans and Fish. Marine Biology 27, 75-88.

Andersson J.T. and Bobinger S. (1992). Polycyclic Aromatic Sulfur Heterocycles. II. Photochemical Oxidation of Benzo[B]Thiophene in Aqueous Solution. Chemosphere 24 (4), 383-389.

Amstutz D.E., Samuels W.B., and Banks A.D. (1984). An Oil Spill Risk Analysis for the South Atlantic Lease Sale 90. Minerals Management Service OS Report MMS 84-0037.

Aravamudan K.S. and Lt. Marsh G. (1981). Simplified Models to Predict the Breakup of Oil on Rough Seas. Proceedings of the 1981 Oil Spill Conference. API. 313-322.

Atlas R.M., Boehm P.D., and Calder J.A. (1981). Chemical and Biological Weathering of Oil, from the Amoco Cadiz Spillage, within the Littoral Zone. Estuarine, Coastal and Shelf Science 12, 589-608.

Beer T., Humphries R.B., and Bouwhuis R. (1983). Modelling Nearshore Oil Slick Trajectories. Marine Pollution Bulletin 14 (4), 141-144.

Berridge S.A., Dean R.A., Fallows R.G, and Fish A. (1968). The Properties of Persistent Oils at Sea. Journal of the Institute of Petroleum 54 (539), 300-307.

Berridge S.A., Thew M.T., and Loriston-Clarke A.G. (1968). The Formation and Stability of Emulsions of Water in Crude Petroleum and Similar Stocks. Journal of the Institute of Petroleum 54 (539), 333-357.

Betancourt O.J. and McLean A.Y. (1973). Changes in Chemical Composition and Physical Properties of a Heavy Residual Oil Weathering under Natural Conditions. Journal of the Institute of Petroleum 59 (569), 223-230.

Blackman R.A.A. and Law R.J. (1980a). The Elena V Oil Spill: Fate and Effects of the Oil Over the First Twelve Months Part I Oil in Waters and Sediments. Marine Pollution Bulletin 11, 199-204. 
Blackman R.A.A. and Law R.J. (1980b). The Elena V Oil Spill: Fate and Effects of the Oil Over the First Twelve Months Part II Biological Effects. Marine Pollution Bulletin 11, 217220.

Bobra M., Fingas M., and Tennyson E. (1992). When Oil Spills Emulsify. Chemtech April 1992, 236-241.

Boehm P.D. and Quinn J.G. (1973). Solubilization of Hydrocarbons by the Dissolved Organic Matter in Sea Water. Geochimica et Cosmochimica Acta 37, 2459-2477.

Bohon R.L. and Claussen W.F. (1951). The Solubility of Aromatic Hydrocarbons in Water. Journal of Physical Chemistry 73, 1571-1578.

Borthwick A.G.L. and Joynes S.A. (1992). Laboratory Study of Oil Slick Subjected to Nearshore Circulation. Journal of Environmental Engineering 118 (6), 905-922.

Boylan D.B. and Tripp B.W. (1971). Determination of Hydrocarbons in Seawater Extracts of Crude Oil and Crude Oil Fractions. Nature 230, 44-47.

Bridié A.L., Wanders Th. E., Zegveld W., and Van Der Heijde H.B. (1980). Formation, Prevention and Breaking of Sea Water in Crude Oil Emulsions "Chocolate Mousses". Marine Pollution Bulletin 11, 343-348.

Buchanan I. and Hurford N. (1988). Methods for Predicting the Physical Changes in Oil Spilt at Sea. Oil and Chemical Pollution 4, 311-328.

Burns K.A. (1993). Evidence for the Importance of Including Hydrocarbon Oxidation Products in Environmental Assessment Studies. Marine Pollution Bulletin 26 (2), 77-85.

Burwood R. and Speers G.C. (1974). Photo-oxidation as a Factor in the Environmental Dispersal of Crude Oil. Estuarine and Coastal Marine Science 2, 117-135.

Davis S.J. and Gibbs C.F. (1975). The Effect of Weathering on a Crude Oil Residue Exposed at Sea. Water Research 9, 275-285.

Delvigne G.A.L. and Sweeney C.E. (1986). Natural Dispersion of Oil. Attachment to Minerals Management Service Study MMS 87-111, Measurement of the Vertical Turbulent Dispersion and Diffusion of Oil Droplets and Oiled Particles.

Delvigne G.A.L., Roelvink J.A., and Sweeny C.E. (1986). Research on Vertical Turbulent Dispersion of Oil Droplets and Oiled Particles: Literature Review, Report No. Z-75-1, Engineering Hydraulics Inc. to Department of the Interior Minerals Management Service. 
Ehrhardt M. and Douabul A. (1989). Dissolved Petroleum Residues and Alkyl-benzene Photo-oxidation Products in the Upper Arabian Gulf. Marine Chemistry 26, 363-370.

Elliott A.J., Hurford N., and Penn C.J. (1986). Shear Diffusion and the Spreading of Oil Slicks. Marine Pollution Bulletin 17 (7), 308-313.

Engineering Hydraulics Inc. (1986). Research on Vertical Turbulent Dispersion of Oil Droplets and Oiled Particles: Literature Review. Minerals Management Service Study MMS 86-0029.

Feldman M.H. (1973). Petroleum Weathering: Some Pathways, Fate, and Disposition on Marine Waters. National Environmental Research Center, EPA 660/3-73-013 September 1973.

Exxon Production Research Company (1989). How Crude Oil acts in the Sea. Oil and Gas Journal April 10, 1989

Fishbein L. (1984). An Overview of Environmental and Toxicological Aspects of Aromatic Hydrocarbons, I.Benzene. The Science of the Total Environment 40, 189-218.

Forrester W.D. (1971). Distribution of Suspended Oil Particles Following the Grounding of the Tanker Arrow. Journal of Marine Research 29, 151-170.

Frankenfeld J.W. (1973). Weathering of Oil at Sea. Final Report to the U.S. Coast Guard. Report No. CG-D-7-75.

Frankenfeld J.W. (1981). Factors Governing the Fate of Oil at Sea: Variations in the Amounts and Types of Dissolved or Dispersed Materials During the Weathering Process. Proceedings of the 1981 Spill Conference, 485-495.

Freegarde M., Hatchard C.G., and Parker C.A. (1971). Oil Spilt at Sea: Its Identification, Determination and Ultimate Fate. Laboratory Practice 20 (1), 35-40.

Gala W.R. and Giesy J.P. (1992). Photo-induced Toxicity of Anthracene to the Green Alga, Selenastrum capricornutum. Archives of Environmental Contamination and Toxicology 23, 316-323.

Geraci J.R. and St. Aubin D.J. (1988). Synthesis of Effects of Oil on Marine Mammals. Department of the Interior, Minerals Management Service, OCS Study MMS 88-0049.

Jaffe R. (1991). Fate of Hydrophobic Organic Pollutants in the Aquatic Environment: A Review. Environmental Pollution 69, 237-257. 
Jeffery P.G. (1981). Large Scale Experiments on the Spreading of Oil at Sea and its Disappearance by Natural Factors. Proceedings of the 1981 Oil Spill Conference, API, 469474.

Johansson S., Larsson U., and Boehm P. (1980). The Tsesis Oil Spill: Impact on the Pelagic Ecosystem. Marine Pollution Bulletin 11 (10), 284-292.

Hansen H.P. (1975). Photochemical Degredation of Petroleum Hydrocarbon Surface Films on Seawater. Marine Chemistry 3, 183-195.

Harrison W., Winnik M.A., Kwong P.T.Y., and Mackay D. (1975). Crude Oil Spills: Disappearance of Aromatic and Aliphatic Components from Small Sea-Surface Slicks. Environmental Science and Technology 9 (3), 231-234.

Hermann R.B. (1972). Theory of Hydrophobic Bonding. II. The Correlation of Hydrocarbon Solubility in Water with Solvent Cavity Surface Area. The Journal of Physical Chemistry 76 (19), 2754-2759.

Huang J.C. (1983). A Review of the State of the Art of Oil Spill Fate/Behavior Models. Proceedings of the 1983 Oil Spill Conference, API, 313-322.

Klein A.E. and Pilpel N. (1974). The Effects of Artificial Sunlight upon Floating Oils. Water Research 8, 79-83.

Kuiper J., De Wilde P., and Wolff W. (1984). Effects of an Oil Spill in Outdoor Model Tidal Flat Ecosystems. Marine Pollution Bulletin 15 (3), 102-106.

Lange P. and Hühnerfuss H. (1978). Drift Response to Monomolecular Slicks to Wave and Wind Action. Journal of Physical Oceanography 8, 142-150.

Larson R.A., Hunt L.L., and Blankenship D.W. (1977). Formation of Toxic Products from a \#2 Fuel Oil by Photooxidation. Environmental Science and Technology 11 (5), 492-496.

Larson R.A., Bott T.L., Hunt L.L., and Rogenmuser K. (1979). Photooxidation Products of a Fuel Oil and Their Antimicrobial Activity. Environmental Science and Technology 13 (18), 965-969.

Lee D.W. and Downum K.R. (1991). The Spectral Distribution of Biologically Active Solar Radiation at Miami, Florida, USA. International Journal of Biometeorology 35, 48-54.

Lee W.Y., Morris A., and Boatwright D. (1980). Mexican Oil Spill: A Toxicity Study of Oil Accommodated in Seawater on Marine Invertebrates. Marine Pollution Bulletin 11, 231-234. 
Leech M.V. and Walker M.I. (1992). Predicting the Fate of Oil Spilt at Sea. Underwater Technology 18 (3), 22-28.

Lehr W.J., Cekirge H.M., Fraga R.J., and Belen M.S. (1984). Empirical Studies of the Spreading of Oil Spills. Oil and Petrochemical Pollution 2 (1), 7-11.

Lehr W.J., Fraga R.J., Belen M.S., and Cekirge H.M. (1984). A New Technique o Estimate Initial Spill Size Using a Modified Fay-type Spreading Formula. Marine Pollution Bulletin 15 (9), 326-329.

Lynch B.W.J. (1987). Fate of Crude Oil at Sea and the Natural Dispersion of Crude Oils and Water-in-Oil Emulsion: Results of Experiments using a Laboratory Test Tank and FreeFloating Rings at Sea. Warren Spring Laboratory. NTIS PB88-198726. ISBN 0-85624-456-2.

Mackay D., Bobra A., Shiu W.Y., and Yalkowsky S.H. (1980). Relationships between Aqueous Solubility and Octanol-Water Partition Coefficients. Chemosphere 9, 701-711.

Mackay D., Paterson S., Boehm P.D., and Fiest D.L. (1981). Physical-Chemical Weathering of Petroleum Hydrocarbons from the IXTOC-I Blowout--Chemical Measurements and a Weathering Model. Proceedings of the 1981 Oil Spill Conference, API, 453-460.

Mackay D. and Shiu W.Y. (1976). Aqueous Solubilities of Weathered Northern Crude Oils. Environmental Contamination and Toxicology 15 (1), 101-109.

Mackay D., Stiver W., and Lt. Cdr. Tebeau P.A. (1983). Testing of Crude Oils and Petroleum Products for Environmental Purposes. Proceedings of the 1983 Oil Spill Conference, 331337.

Mackay S.D.M., McLean A.Y., Betancourt O.J., and Johnson B.D. (1973). The Formation of Water-in-Oil Emulsions Subsequent to an Oil Spill. Journal of the Institute of Petroleum 59 (568), 164-171.

Mayo D.W., Page D.S., Cooley J., Sorenson E., Bradley F., Gilfillan E.S., and Hanson S.A. (1978). Weathering Characteristics of Petroleum Hydrocarbons Deposited in Fine Clay Marine Sediments, Searsport, Maine. J. Fish. Board Can.35, 552-562.

McAuliffe C.D. (1966). Solubility in Water of Paraffin, Cycloparaffinic, Olefin, Acetylene, Cycloolefin, and Aromatic Hydrocarbons. The Journal of Physical Chemistry 70 (4), 12671275. 
McAuliffe C.D. (1973). The Environmental Impact of an Offshore Oil Spill. Background Information for Ocean Affairs Board Workshop on Inputs, Fates and Effects of Petroleum in the Marine Environment 21-25 May 1973, 225-236.

McAuliffe C.D. (1976a). Dispersal and Alteration of Oil Discharged on a Water Surface. Fate and Effects of Petroleum Hydrocarbons in Marine Ecosystems and Organisms, Proceedings of a Symposium November 10-12, 1976, Pergamon Press, 19-35.

McAuliffe C.D. (1976b). Evaporation and Solution of C2 to C10 Hydrocarbons from Crude Oils on the Sea Surface. Fate and Effects of Petroleum Hydrocarbons in Marine Ecosystems and Organisms, Proceedings of a Symposium November 10-12, 1976, Pergamon Press, 363371.

Milgram N., Donnelly R.G., Van Houten R.J., and Camperman J.M. (1978). Effects of Oil Slick Properties on the Dispersion of Floating Oil into the Sea. Final Report: Massachusetts Institute of Technology to Office of Research and Development United States Coast Guard, Report No. CG-D-64-78.

Minerals Management Service OCS Study MMS 87-111 (1987). Measurement of Vertical Turbulent Dispersion and Diffusion of Oil Droplets and Oiled Particles. Engineering Hydraulics, Inc. / Delft Hydraulics Laboratory.

National Academy of Sciences (1973). Background Papers for a Workshop on Inputs, Fates, and Effects of Petroleum in the Marine Environment, Vol. I/II, NTIS AD 783990.

Owens E.H. (1978). Mechanical Dispersal of Oil Stranded in the Littoral Zone. J. Fish. Res. Board Can. 35, 563-572.

Page D.S., Foster J.C., Hotham J.R., Pendergast E., Hebert S., Gonzalez L., Gilfillan E.S., Hanson S.A., Gerber R.P., and Vallas D. (1983). Long Term Fate of Dispersed and Undispersed Crude Oil in Two Nearshore Test Spills. Proceedings of the 1983 Oil Spill Conference. API, 465-471.

Patton J.S., Rigler M.W., Boehm P.D., and Fiest D.L. (1981). IXTOC I Oil Spill: Flaking of Surface Mousse in the Gulf of Mexico. Nature 290, 235-238.

Payne J.R., Kirstein B.E., McNabb Jr. G.D., and Lambach J.L. (1983). Multi-variate Analysis of Petroleum Hydrocarbon Weathering in the Subarctic Marine Environment. Proceedings of the 1983 Oil Spill Conference. API. 423-434.

Payne J.R. and McNabb, Jr. G.D. (1985). Weathering of Petroleum in the Marine Environment. Journal of the Marine Technological Society 18 (3), 24-42. 
Payne J.R. and Phillips C.R. (1985a). Photochemistry of Petroleum in Water. Environmental Science and Technology 19 (7), 569-579.

Payne J.R. and Phillips C.R. (1985b). Petroleum Spills in the Marine Environment The Chemistry and Formation of Water in Oil Emulsions and Tar Balls. Lewis Publishers, Inc., Chelsea, Michigan.

Pengerud B., Thingstad F., Tjessem K., and Aaberg A. (1984). Photo-induced Toxicity of North Sea Crude Oils Toward Bacterial Activity. Marine Pollution Bulletin 15 (4), 142-146.

Raj P.P.K. (1977). Theoretical Study to Determine the Sea State Limit for the Survival of Oil Slicks on the Ocean. Report to the Office of Research and Development, United States Coast Guard. Report No. CG-D-90-77.

Raj P.P.K. and Griffiths R.A. (1979). The Survival of Oil Slicks on the Ocean as a Function of Sea State Limit. Proceedings of the 1979 Oil Spill Conference, API, 719-724.

Reed M., Gundlach E., and Kana T. (1989). A Coastal Zone Oil Spill Model: Development and Sensitivity Studies. Oil and Chemical Pollution 5, 411-449.

Singh A.K. and Gaur J.P. (1990). Effects of Petroleum Oils and their Paraffinic, Asphaltic, and Aromatic Fractions on Photosynthesis and Respiration of Microalgae. Ecotoxicology and Environmental Safety 19, 8-16.

Siron R., Pelletier E., Delille D., and Roy S. (1993). Fate and Effects of Dispersed Crude Oil Under Icy Conditions Simulated in Mesocosms. Marine Environmental Research 35, 273302.

Siron R., Giusti G., Berland B., Morales-Loo R., and Pelletier E. (1991). Water Soluble Petroleum Compounds: Chemical Aspects and Effects on the Growth of Microalgae. The Science of the Total Environment 104, 221-227.

Sivadier H.O. and Mikolaj P.G. (1981). Measurement of Evaporation Rates from Slicks on the Open Sea. Proceedings of the 1981 Oil Spill Conference, API, 475-483.

Shaw D.G. (1976). Hydrocarbons in the Water Column. Fate and Effects of Petroleum Hydrocarbons in Marine Ecosystems and Organisms, Proceedings of a Symposium November 10-12, 1976, Pergamon Press, 8-18.

Soler M., Grimalt J.O., Albaiges J., Mendez J., and Marino M. (1989). Distribution of Aliphatic, Aromatic and Chlorinated Hydrocarbons in Mussels from the Spanish Coast (Galicia). An Assessment of Pollution Parameters. Chemosphere 19 (10/11), 1489-1498. 
Spaulding M.L. (1988). A State of the Art Review of Oil Spill Trajectory and Fate Modeling. Oil and Chemical Pollution 4, 39-55.

Strand J.A., Cullinan V.I., Crecelius E.A., Fortman T.J., Citterman R.J., and Fleischmann M.L. (1992). Fate of Bunker C Fuel Oil in Washington Coastal Habitats Following the December 1988 NESTUCCA Oil Spill. Northwest Science 66 (1), 1-14.

Sutton C. and Calder J.A. (1974). Solubility of Higher Molecular Weight n-Paraffins in Distilled Water and Seawater. Environmental Science and Technology 8 (7), 654-657.

Thingstad T. and Pengerud B. (1983). The Formation of "Chocolate Mousse" from Statfjord Crude Oil and Seawater. Marine Pollution Bulletin 14 (6), 214-216.

Thominette F. and Verdu J. (1984). Photo-oxidative behaviour of Crude Oils Relative to Sea Pollution. (Part I)- Comparative Study of Various Crude Oils and Model Systems. Marine Chemistry 15, 91-104.

Thominette F. and Verdu J. (1984). Photo-oxidative behaviour of Crude Oils Relative to Sea Pollution. (Part II)- Photo-induced Phase Separation. Marine Chemistry 15, 105-15.

Venkatesh S. (1988). The Oil Spill Behaviour Model of the Canadian Atmospheric Environment Service Part I: Theory and Model Evaluation. Atmosphere-Ocean 26 (1), 93 108.

Winters K., O'Donnell R., Batterton J.C., and Van Baalen C. (1976). Water Soluble Components of Four Fuel Oils: Chemical Characterization and Effects on Growth of Microalgae. Marine Biology 36, 269-276.

Wolfe D.A (1978). The Amoco Cadiz Oil Spill, A Summary of Observations Made by U.S. Scientists 23 March - 10 May, 1978. Marine Pollution Bulletin

Yaws C.L. (1992). Hydrocarbon Solubility in Salt Water. Pollution Engineering September 15, 1992, 46-50.

Zürcher F. and Thüer M. (1978). Rapid Weathering Processes of Fuel Oil in Natural Waters: Analyses and Interpretations. Environmental Science and Technology 12 (7), 838-845. 


\subsection{APPENDICES}


9.1 APPENDIX A

ARABIAN CRUDE OIL (API 28) COMPOSITIONAL DATA TBP AND DISTILLATION DATA 

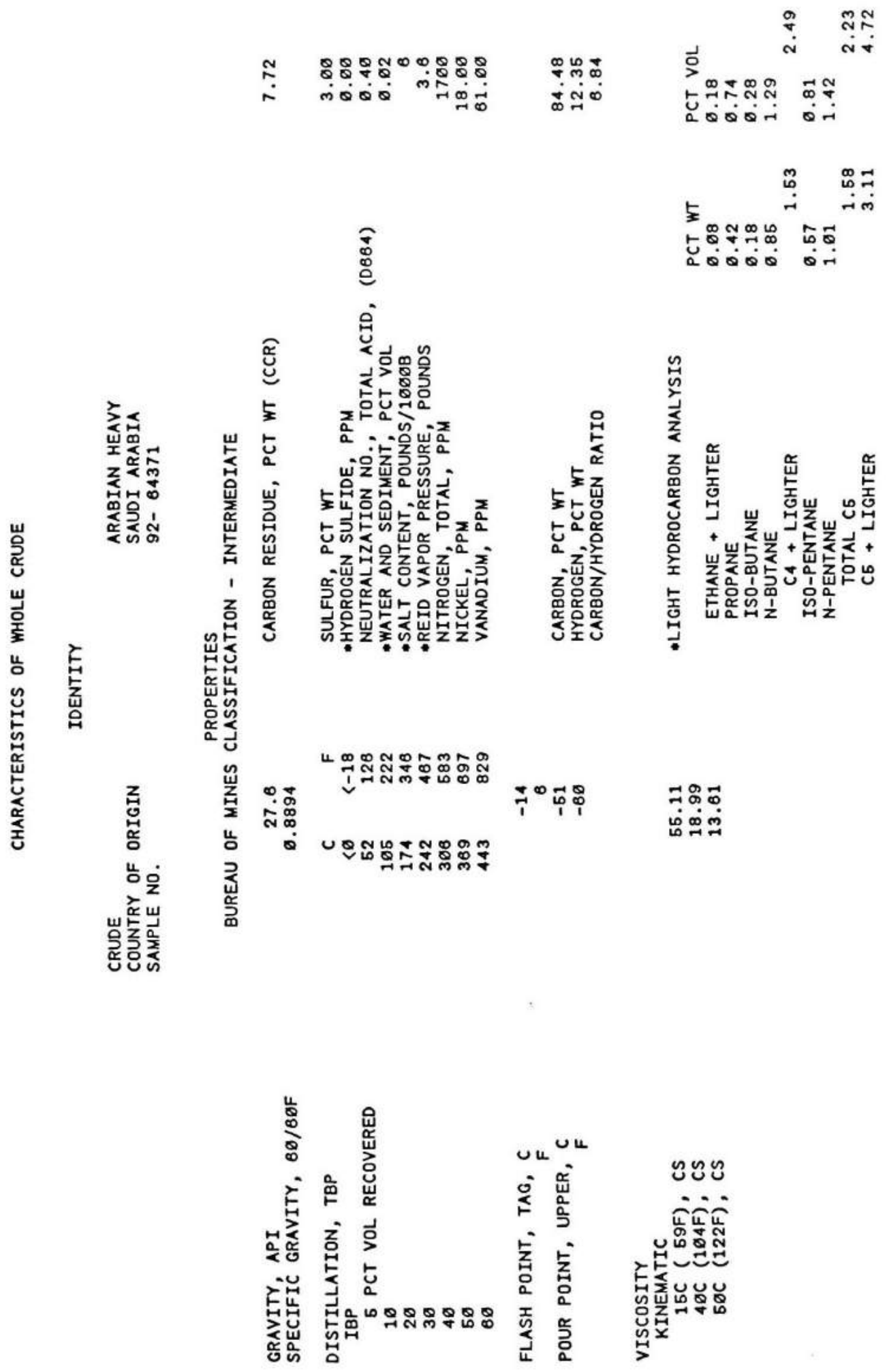
YIELDS AND GRAVITIES OF NARROW FRACTIONS FROM TBP DISTILLATION OF ARABIAN HEAVY CRUDE

\begin{tabular}{|c|c|c|c|c|c|c|c|c|c|c|c|}
\hline CUT NO. & $\begin{array}{l}\text { CUT }{ }^{\mathrm{TB}} \mathrm{P} \\
\text { DEG } F\end{array}$ & $\begin{array}{l}P \\
\text { OINT } \\
\text { DEG C }\end{array}$ & $\begin{array}{r}\text { M } \\
\text { TBP } \\
\text { DEG F }\end{array}$ & $\begin{array}{l}\text { TEMP } \\
\text { DEG C }\end{array}$ & $\begin{array}{l}\text { YIELD } \\
\text { PCT } \\
\text { WT }\end{array}$ & $\begin{array}{l}\text { SUM } \\
\text { YIELD } \\
\text { PCT } \\
W T\end{array}$ & $\begin{array}{l}\text { YIELD } \\
\text { PCT } \\
\text { VOL }\end{array}$ & $\begin{array}{l}\text { SUM } \\
\text { YIELD } \\
\text { PCT } \\
\text { VOL }\end{array}$ & $\begin{array}{l}\text { MID } \\
\text { PCT } \\
\text { VOL }\end{array}$ & $\begin{array}{l}\text { GRAVITY } \\
\text { DEG API }\end{array}$ & $\begin{array}{c}\text { SPECIFIC } \\
\text { GRAVITY } \\
6 \varnothing / 6 \emptyset \\
\text { DEG F }\end{array}$ \\
\hline $\begin{array}{l}\mathrm{C}_{1}+\mathrm{C} 2 \\
\mathrm{C} 3 \\
\mathrm{IC} 4 \\
\mathrm{NC} 4\end{array}$ & & & & & $\begin{array}{l}0.08 \\
0.42 \\
0.18 \\
6.86\end{array}$ & $\begin{array}{l}6.68 \\
6.56 \\
6.68 \\
1.53\end{array}$ & $\begin{array}{l}0.18 \\
0.74 \\
6.28 \\
1.29\end{array}$ & $\begin{array}{l}0.18 \\
6.92 \\
1.20 \\
2.49\end{array}$ & $\begin{array}{l}6.09 \\
6.55 \\
1.68 \\
1.85\end{array}$ & $\begin{array}{l}222.2 \\
147.2 \\
119.8 \\
110.6\end{array}$ & $\begin{array}{l}0.4001 \\
0.5077 \\
0.5631 \\
0.5845\end{array}$ \\
\hline $\begin{array}{l}\text { ICE } \\
\text { NCE }\end{array}$ & 120 & 48 & & & $\begin{array}{l}0.67 \\
1.61\end{array}$ & $\begin{array}{l}2.10 \\
3.11\end{array}$ & $\begin{array}{l}6.81 \\
1.42\end{array}$ & $\begin{array}{l}3.30 \\
4.72\end{array}$ & $\begin{array}{l}2.90 \\
4.01\end{array}$ & $\begin{array}{l}85.6 \\
92.7\end{array}$ & $\begin{array}{l}0.6247 \\
0.6311\end{array}$ \\
\hline $\begin{array}{l}3 \\
4 \\
5 \\
6 \\
7\end{array}$ & $\begin{array}{l}195 \\
244 \\
286 \\
329 \\
386\end{array}$ & $\begin{array}{r}91 \\
118 \\
141 \\
165 \\
193\end{array}$ & $\begin{array}{l}158 \\
220 \\
265 \\
368 \\
355\end{array}$ & $\begin{array}{r}70 \\
164 \\
129 \\
153 \\
179\end{array}$ & $\begin{array}{l}2.67 \\
2.82 \\
2.89 \\
2.89 \\
3.67\end{array}$ & $\begin{array}{r}5.68 \\
8.66 \\
11.39 \\
14.38 \\
18.65\end{array}$ & $\begin{array}{r}.3 .36 \\
3.53 \\
3.48 \\
3.51 \\
.4 .19\end{array}$ & $\begin{array}{r}8.68 \\
11.61 \\
15.69 \\
18.68 \\
22.79\end{array}$ & $\begin{array}{r}6.40 \\
9.85 \\
13.35 \\
16.85 \\
20.76\end{array}$ & $\begin{array}{l}76.6 \\
67.2 \\
60.4 \\
54.9 \\
56.2\end{array}$ & $\begin{array}{l}0.6860 \\
6.7121 \\
0.7374 \\
6.7591 \\
0.7788\end{array}$ \\
\hline $\begin{array}{r}8 \\
9 \\
10 \\
11 \\
12\end{array}$ & $\begin{array}{l}426 \\
465 \\
566 \\
547 \\
588\end{array}$ & $\begin{array}{l}216 \\
241 \\
263 \\
286 \\
369\end{array}$ & $\begin{array}{l}468 \\
443 \\
486 \\
527 \\
568\end{array}$ & $\begin{array}{l}204 \\
228 \\
252 \\
275 \\
298\end{array}$ & $\begin{array}{l}3.17 \\
3.18 \\
3.28 \\
3.37 \\
3.38\end{array}$ & $\begin{array}{l}21.22 \\
24.46 \\
27.68 \\
31.63 \\
34.41\end{array}$ & $\begin{array}{l}3.53 . \\
3.51 . \\
3.51 \\
3.56 \\
3.54\end{array}$ & $\begin{array}{l}26.32 \\
29.83 \\
33.34 \\
36.98 \\
46.44\end{array}$ & $\begin{array}{l}24.56 \\
28.68 \\
31.59 \\
35.12 \\
38.67\end{array}$ & $\begin{array}{l}45.6 \\
44.5 \\
39.8 \\
36.2 \\
35.2\end{array}$ & $\begin{array}{l}0.799 \emptyset \\
6.804 \emptyset \\
.0826 \emptyset \\
\varnothing .8438 \\
0.8488\end{array}$ \\
\hline $\begin{array}{l}13 \\
14 \\
15 \\
16 \\
17\end{array}$ & $\begin{array}{l}628 \\
689 \\
716 \\
768 \\
869\end{array}$ & $\begin{array}{l}331 \\
354 \\
377 \\
469 \\
432\end{array}$ & $\begin{array}{l}688 \\
649 \\
696 \\
739 \\
789\end{array}$ & $\begin{array}{l}320 \\
343 \\
368 \\
393 \\
421\end{array}$ & $\begin{array}{l}3.44 \\
3.52 \\
3.62 \\
4.69 \\
3.63\end{array}$ & $\begin{array}{l}37.85 \\
41.37 \\
44.99 \\
49.59 \\
52.62\end{array}$ & $\begin{array}{r}3.54 \\
3.55 \\
3.61 \\
4.51 \\
.2 .95\end{array}$ & $\begin{array}{l}43.88 \\
47.53 \\
51.14 \\
55.65 \\
58.68\end{array}$ & $\begin{array}{l}42.21 \\
45.76 \\
49.34 \\
53.46 \\
57.13\end{array}$ & $\begin{array}{l}32.6 \\
29.6 \\
26.8 \\
24.6 \\
23.6\end{array}$ & $\begin{array}{l}0.8628 \\
0.8816 \\
6.8939 \\
\varnothing .9665 \\
0.9123\end{array}$ \\
\hline $\begin{array}{l}18 \\
19 \\
26 \\
21 \\
22\end{array}$ & $\begin{array}{r}850 \\
890 \\
931 \\
976 \\
1000\end{array}$ & $\begin{array}{l}454 \\
477 \\
499 \\
524 \\
538\end{array}$ & $\begin{array}{l}830 \\
87 \emptyset \\
911 \\
954 \\
988\end{array}$ & $\begin{array}{l}443 \\
468 \\
488 \\
512 \\
531\end{array}$ & $\begin{array}{l}3.62 \\
3.68 \\
3.68 \\
3.67 \\
1.46\end{array}$ & $\begin{array}{l}55.64 \\
58.76 \\
61.78 \\
64.85 \\
66.31\end{array}$ & $\begin{array}{l}2.92 \\
2.92 \\
2.92 \\
2.88 \\
1.36\end{array}$ & $\begin{array}{l}61.52 \\
64.44 \\
67.36 \\
70.24 \\
71.60\end{array}$ & $\begin{array}{l}6 \varnothing .68 \\
62.98 \\
65.90 \\
68.86 \\
7 \varnothing .92\end{array}$ & $\begin{array}{l}22.1 \\
20.2 \\
19.3 \\
18.2 \\
16.6\end{array}$ & $\begin{array}{l}0.9212 \\
\emptyset .9328 \\
0.9383 \\
6.9452 \\
\emptyset .9554\end{array}$ \\
\hline 23 & 1160 & 627 & 1080 & 582 & 10.67 & 76.98 & .9 .68 & 81.28 & 76.44 & 12.8 & ஏ. $98 \varnothing 8$ \\
\hline BOTTOMS & & & & & 23.04 & 100.02 & 19.26 & 180.54 & & 1.5 & 1.0839 \\
\hline
\end{tabular}

NOTE: FRACTIONS ARE DEFINED BY LABORATORY TRUE BOILING POINT (TBP) OF WHOLE CRUDE AND ARE NOT NECESSARILY REPRESENTATIVE OF COMMERCIAL DISTILLATION. 


\begin{tabular}{||c|c|c|c|c|c||}
\hline \multicolumn{7}{|c||}{ CRUDE OIL SAMPLE CHARACTERISTICS } \\
\hline \hline NAME & COUNTRY & $\begin{array}{c}\% \\
\text { VOLUME } \\
\text { BOILING } \\
80-170^{\circ} \mathrm{C}\end{array}$ & $\begin{array}{c}\% \\
\text { VOLUME } \\
\text { BOILING } \\
218-340^{\circ} \mathrm{C}\end{array}$ & $\begin{array}{c}\% \\
\text { VOLUME } \\
\text { BOILING } \\
400+{ }^{\circ} \mathrm{C}\end{array}$ & API \\
\hline STATFJORD & NORWAY & 23.01 & 26.23 & 29.00 & 38 \\
\hline $\begin{array}{c}\text { ARABIAN } \\
\text { HEAVY }\end{array}$ & $\begin{array}{c}\text { SAUDI } \\
\text { ARABIA }\end{array}$ & 18.07 & 21.21 & 44.89 & 28 \\
\hline BCF-17 & VENEZUELA & 5.58 & 17.67 & 63.39 & 17 \\
\hline \hline
\end{tabular}

\begin{tabular}{|c|c||}
\hline \multicolumn{2}{|c||}{ CRUDE OIL SULFUR CONTENT } \\
\hline \hline NAME & $\%$, WEIGHT \\
\hline \hline STATFJORD & 0.28 \\
\hline ARABIAN HEAVY & 3.0 \\
\hline BCF-17 & 2.3 \\
\hline
\end{tabular}




\section{A Quick Review Of Cone/Plate Geometry}

Cone and Plate geometry, as illustrated, is the fixation of a conical vertex perpendicular to and in point contact with a flat plate. When the cone is made very obtuse ( $\theta$ less than $\left.4^{\circ}\right)$ and rotated at constant speed $(\omega)$, precise viscosity measurements are obtained at absolute and uniform values of shearing rate and stress.

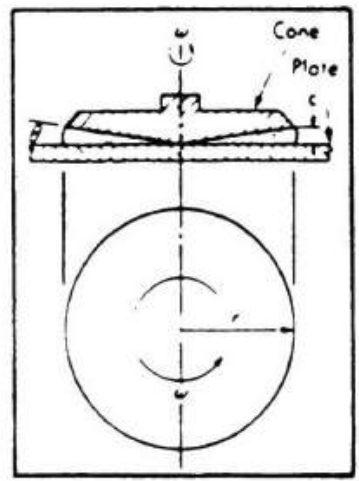

Viscosity (poise) is the ratio of shear stress to shear rate. Shear stress is related to the summation of torque $(\mathrm{T})$ over the conical surface. Shear rate is related to the cone rotational speed $(\omega)$, and gap width (c) at any radial

distance ( $r$ ) from the center of the rotating cone. The ratio of ( $\omega r)$ and $(c)$ is a constant for any value of $(r)$. Since (c) is a maximum at cone radius ( $r$ ), the shear rate is related to $(\omega)$ and sine $(\theta)$.

For the Wells-Brookfield Cone/Plate Viscometer, the mathematical relationships are:

Shear Stress $\left(\right.$ dynes $\left.\mathrm{cm}^{2}\right)=$

$$
\frac{T}{2 / 3 \pi r^{3}}
$$

Shear Rate $\left(\operatorname{Sec}^{-1}\right)=$

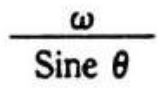

$$
\underset{\begin{array}{c}
\text { Viscosity } \\
\text { (mPntipoise) } \\
\text { (mPa) }
\end{array}}{\text { Shear Stress } \times 100}
$$

Where:

$\mathrm{T}=\%$ Full Scale Torque (dyne-cm)

$\mathrm{r}=$ Cone Radius $(\mathrm{cm})$

$\omega=$ Cone Speed (radkec)

$\theta=$ Cone Angle (degrees)

Cone Radius:

CP 40, CP 41, CP $42-2.4 \mathrm{~cm}$

CP 51, CP $52-1.2 \mathrm{~cm}$ 
9.3 APPENDIX C

CALCULATION OF ENERGY DISSIPATION RATE FOR VERTICAL TURBULENT DISPERSION REACTOR 


\section{Calculation of Energy Dissipation Rate for Vertical Turbulent Dispersion Reactor}

$\underline{\text { Stir Pak Speed Control Setting }}$

2

3

4

5

6
* Speed (rpm)

528

853

1203

1519

1820

* Speed determined by Tak-ette Model 1707 hand-held digital tachometer

Selected speed setting for experiments $=3.5$

Rotation speed $=1028 \mathrm{rpm}$

Calculation of Reynolds Number: $N_{R}=D^{2} n \rho / \mu$

$$
\begin{aligned}
& \text { where: } \mathrm{D} \\
& \qquad \begin{aligned}
& \mathrm{n}=\text { propeller diameter (meters) } \\
& \rho=\text { fluid density }\left(\mathrm{kg} / \mathrm{m}^{3}\right) \\
& \mu=\text { fluid viscosity }(\mathrm{kg} / \mathrm{s}-\mathrm{m}) \\
& \mathrm{N}_{\mathrm{R}}=(.0762)^{2}(1028 / 60)(997.0) /\left(0.89 \times 10^{-6}\right) \\
&=111444069 \\
& \text { turbulent conditions prevail } \gg>10,000
\end{aligned}
\end{aligned}
$$

Power equation for propeller mixing (turbulent conditions)

$\mathrm{P}=\mathrm{k} \rho \mathrm{n}^{3} \mathrm{D}^{5}$

$\mathrm{k}=0.32$ for square pitched propellers

$\mathrm{P}=4.122 \mathrm{~W}=3.93 \mathrm{~J} / \mathrm{s}$

if volume of reactor under turbulent conditions is assumed to be $0.06624 \mathrm{~m}^{3}$ (i.e., one-half of the 35-gal volume)

then energy dissipation rate in the turbulence zone is then $3.93 / 0.06624=59.32 \mathrm{~J} / \mathrm{s}-\mathrm{m}^{3}$

energy dissipation rate for deep sea conditions energy dissipation rate for breaking wave conditions

$$
\begin{aligned}
& =10^{-4} \text { to } 10^{-2} \mathrm{~J} / \mathrm{s}-\mathrm{m}^{3} \\
& =1000 \text { to } 10,000 \mathrm{~J} / \mathrm{s}-\mathrm{m}^{3}
\end{aligned}
$$


9.4 APPENDIX D

DYNAMIC VISCOSITY OF WEATHERED ARABIAN CRUDE OIL SAMPLES 
OIL VISCOSITY DATA SHEET weathering period: none

\begin{tabular}{|c|c|c|c|c|c|}
\hline spindle rpm & $\%$ max range & $\begin{array}{c}\text { shear rate } \\
(1 / \mathrm{sec})\end{array}$ & $\begin{array}{l}\text { shear stress } \\
\text { (dynes/cm2) }\end{array}$ & $\begin{array}{l}\text { viscosity } \\
(\mathrm{cP})\end{array}$ & viscosity $\mathrm{x} \% \max$ range \\
\hline 1 & 39.1 & 7.5 & 8.99 & 119.9 & 4688.09 \\
\hline 1.5 & 59.3 & 11.3 & 13.6 & 121.2 & 7187.16 \\
\hline 2 & 75.9 & 15 & 17.4 & 115.3 & 8751.27 \\
\hline 2.5 & 94.1 & 18.8 & 21.7 & 115.8 & 10896.78 \\
\hline & & & & & \\
\hline 1 & 41.5 & & & 126.9 & 5266.35 \\
\hline 1.5 & 57 & & & 116.3 & 6629.1 \\
\hline 2 & 77.7 & & & 119.3 & 9269.61 \\
\hline 2.5 & 94.7 & & & 116.2 & 11004.14 \\
\hline SUMMATION & 539.3 & & & & 63692.5 \\
\hline
\end{tabular}

WEIGHTED
AVERAGE

118.1021695

$118.10 \mathrm{cP}$

\section{Shear Stress vs. Shear Rate}

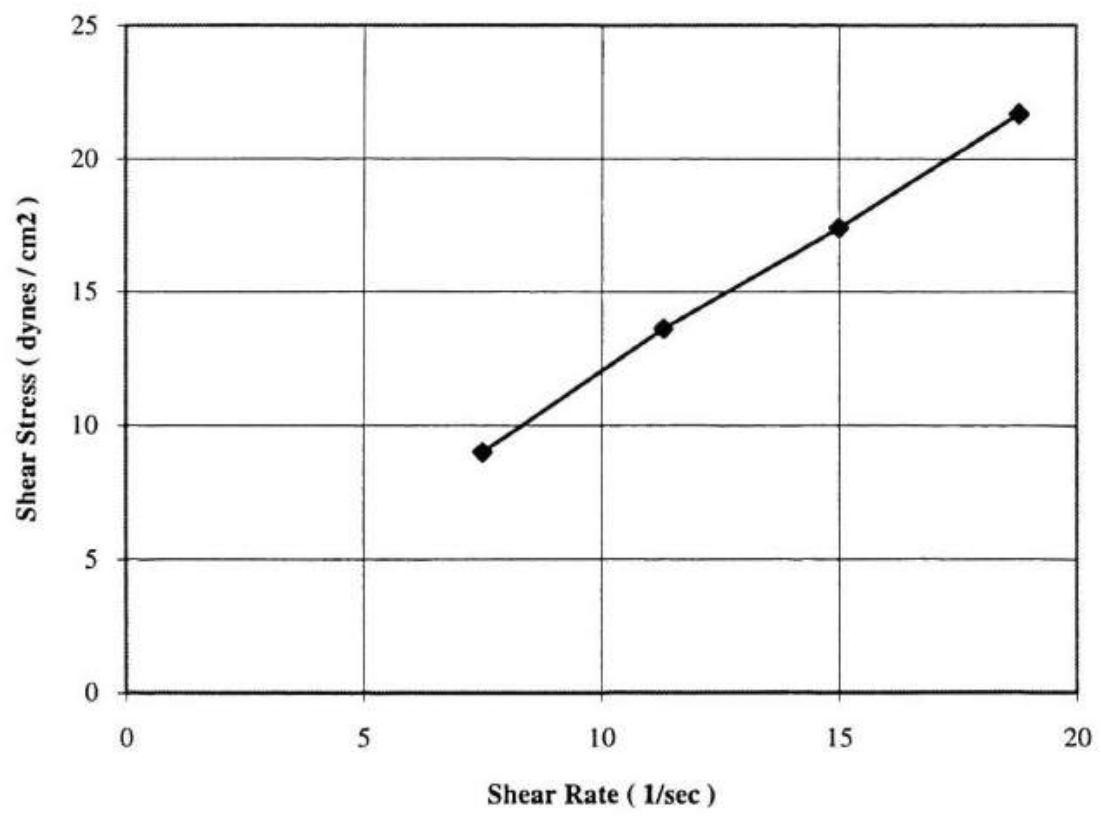


OIL VISCOSITY DATA SHEET weathering period: 4 hours

\begin{tabular}{|c|c|c|c|c|c|}
\hline spindle rpm & $\%$ max range & $\begin{array}{c}\text { shear rate } \\
(1 / \mathrm{sec})\end{array}$ & $\begin{array}{c}\text { shear stress } \\
(\text { dynes } / \mathrm{cm} 2)\end{array}$ & $\begin{array}{l}\text { viscosity } \\
\text { (cP) }\end{array}$ & viscosity $\mathrm{x} \%$ max range \\
\hline 0.3 & 221 & 2.25 & & 2258 & 499018 \\
\hline 0.6 & 37.9 & 4.5 & & 194.2 & 7360.18 \\
\hline 1 & 64.4 & 7.5 & & 197.7 & 12731.88 \\
\hline 1.5 & 97.5 & 11.3 & & 199.1 & 19412.25 \\
\hline 03 & 225 & & & 2200 & 517275 \\
\hline 0.6 & 42 & & & 214.6 & $\frac{517.15}{9013.2}$ \\
\hline 1 & 64 & & & 195.9 & 12537.6 \\
\hline 1.5 & 97.2 & & & 198.4 & 19284.48 \\
\hline SUMMATION & 447.6 & & & & 90502.52 \\
\hline
\end{tabular}

\begin{tabular}{|cc|}
\hline WEIGHTED & 202.1950849 \\
AVERAGE & $202.20 \mathrm{cP}$ \\
& \\
\hline
\end{tabular}


OIL VISCOSITY DATA SHEET weathering period: 8 hours

\begin{tabular}{|c|c|c|c|c|c|}
\hline spindle rpm & $\%$ max range & $\begin{array}{c}\text { shear rate } \\
(1 / \mathrm{sec})\end{array}$ & $\begin{array}{c}\text { shear stress } \\
\text { (dynes/cm2) }\end{array}$ & $\begin{array}{l}\text { viscosity } \\
(\mathrm{cP})\end{array}$ & viscosity $\times \%$ max range \\
\hline 03 & 312 & 225 & 717 & 3188 & 994656 \\
\hline 0.5 & 49.9 & 3.75 & 11.4 & 305.3 & 15234.47 \\
\hline 0.6 & 56 & 4.5 & 12.9 & 286.1 & 16021.6 \\
\hline 1 & 93 & 7.5 & 21.4 & 285.1 & 26514.3 \\
\hline & & & & & \\
\hline 0.3 & 30.4 & & & 310.7 & 9445.28 \\
\hline 0.5 & 45.1 & & & 276.5 & 12470.15 \\
\hline 0.6 & 58.3 & & & 297.9 & 17367.57 \\
\hline 1 & 93.8 & & & 287.8 & 26995.64 \\
\hline SUMMATION & 457.7 & & & & 133995.57 \\
\hline
\end{tabular}

WEIGHTED

AVERAGE

292.7585099

$292.76 \mathrm{cP}$

Shear Stress vs. Shear Rate

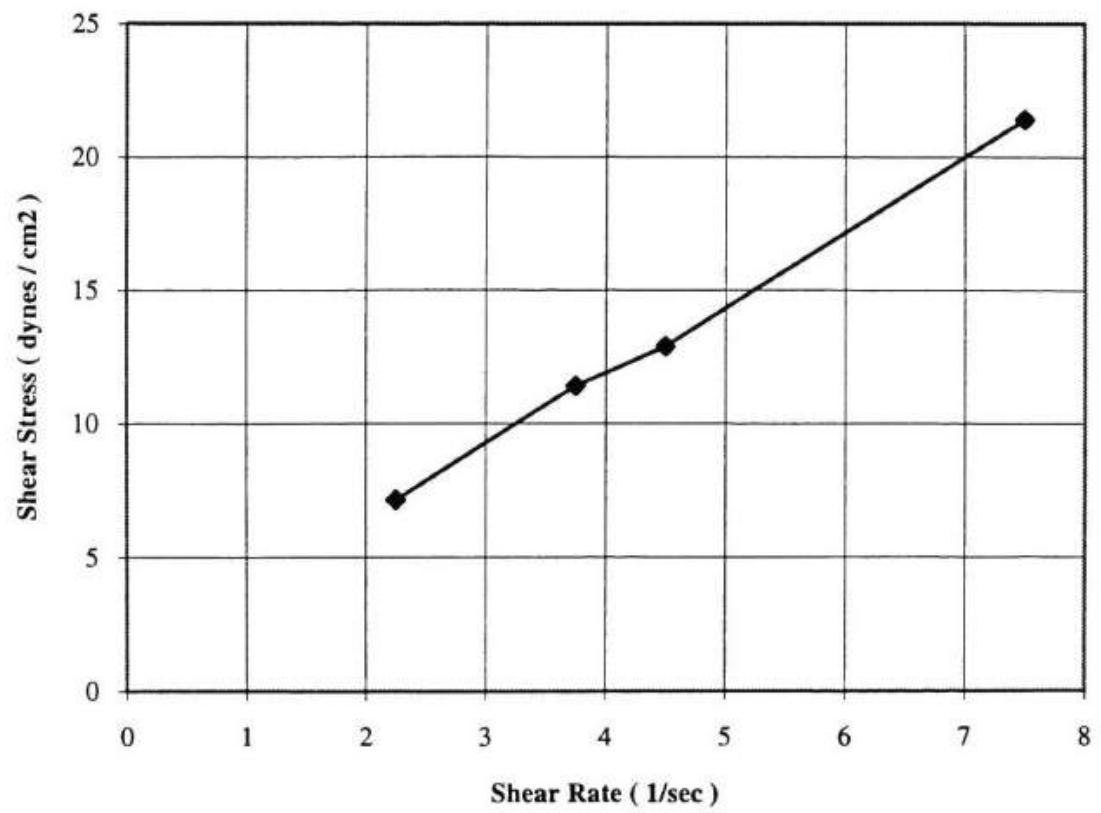


OIL VISCOSITY DATA SHEET weathering period: 24 hours

\begin{tabular}{|c|c|c|c|c|c|}
\hline spindle rpm & $\%$ max range & $\begin{array}{c}\text { shear rate } \\
(1 / \mathrm{sec})\end{array}$ & $\begin{array}{l}\text { shear stress } \\
\text { (dynes/cm2) }\end{array}$ & $\begin{array}{l}\text { viscosity } \\
\text { (cP) }\end{array}$ & viscosity $x \%$ max range \\
\hline 0.3 & 35.3 & 2.25 & 8.12 & 3607 & 12732.71 \\
\hline 0.5 & 53.9 & 3.75 & 12.5 & 331.5 & 17867.85 \\
\hline 0.6 & 66 & 4.5 & 15.2 & 337.2 & 22255.2 \\
\hline & & & & & \\
\hline & & & & & \\
\hline 0.3 & 36.2 & & & 366.9 & 13281.78 \\
\hline 0.5 & 53.4 & & & 327.4 & 17483.16 \\
\hline 0.6 & 64.4 & & & 329 & 21187.6 \\
\hline & & & & & \\
\hline & & & & & \\
\hline SUMMATION & 309.2 & & & & 104808.3 \\
\hline
\end{tabular}

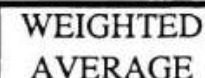

AVERAGE
338.9660414

$338.97 \mathrm{cP}$

\section{Shear Stress vs. Shear Rate}

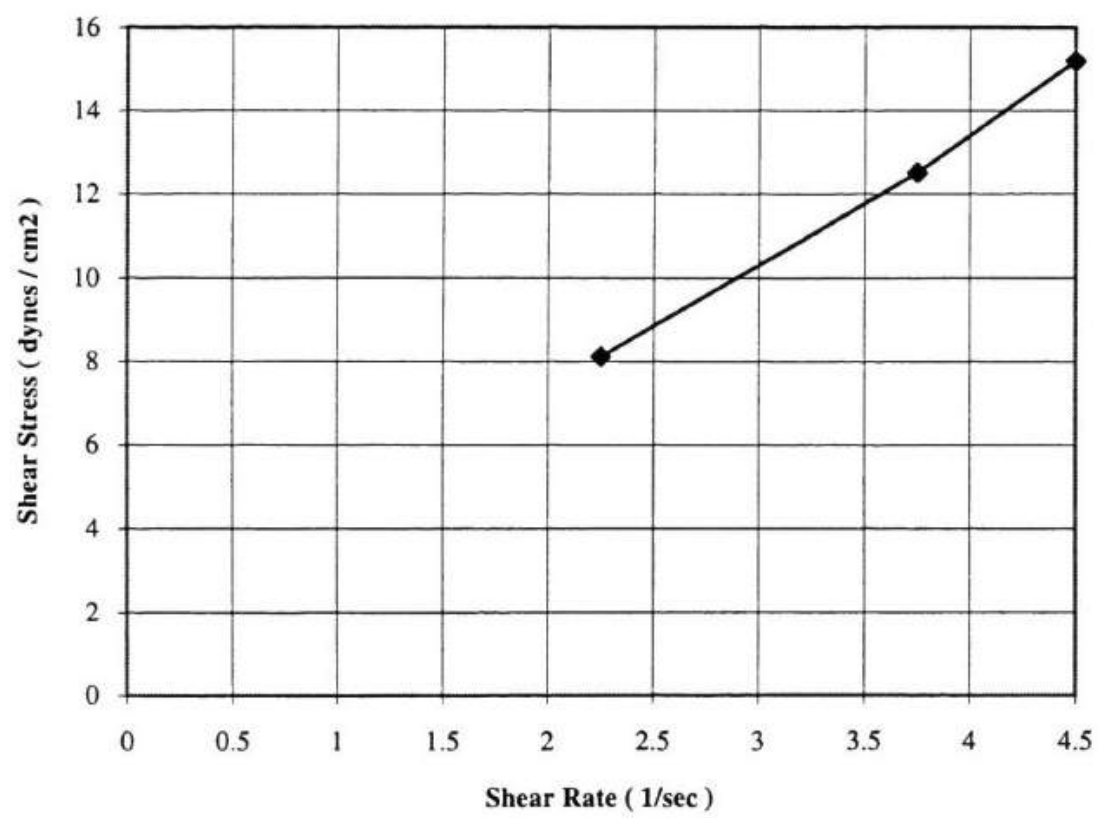


OIL VISCOSITY DATA SHEET weathering period: 4 days

\begin{tabular}{|c|c|c|c|c|c|}
\hline spindle rpm & $\%$ max range & $\begin{array}{c}\text { shear rate } \\
(1 / \mathrm{sec})\end{array}$ & $\begin{array}{c}\text { shear stress } \\
(\text { dynes } / \mathrm{cm} 2)\end{array}$ & $\begin{array}{l}\text { viscosity } \\
(\mathrm{cP})\end{array}$ & viscosity $\mathrm{x} \%$ max range \\
\hline 03 & 567 & 225 & & 5804 & 3200868 \\
\hline 0.5 & 98.7 & 3.75 & & $\frac{500.4}{605.8}$ & 59792.46 \\
\hline & & & & & \\
\hline 0.5 & 97.8 & 3.75 & & 600.2 & 58699.56 \\
\hline & & & & & \\
\hline & & & & & \\
\hline & & & & & \\
\hline & & & & & \\
\hline & & & & & \\
\hline & & & & & \\
\hline SUMMATION & 253.2 & & & & 151400.7 \\
\hline
\end{tabular}

WEIGHTED

AVERAGE

597.9490521

$597.95 \mathrm{cP}$ 
OIL VISCOSITY DATA SHEET weathering period: 4 days

\begin{tabular}{|c|c|c|c|c|c|}
\hline spindle rpm & $\%$ max range & $\begin{array}{c}\text { shear rate } \\
(1 / \mathrm{sec})\end{array}$ & $\begin{array}{c}\text { shear stress } \\
(\text { dynes } / \mathrm{cm} 2)\end{array}$ & $\begin{array}{l}\text { viscosity } \\
(\mathrm{cP})\end{array}$ & viscosity $\mathrm{x} \% \max$ range \\
\hline 0.3 & 91 & 2.25 & & 929.9 & 84620.9 \\
\hline 0.3 & 89.1 & 2.25 & & 910.5 & 81125.55 \\
\hline & & & & & \\
\hline & & & & & \\
\hline & & & & & \\
\hline & & & & & \\
\hline & & & & & \\
\hline & & & & & \\
\hline & & & & & \\
\hline & & & & & \\
\hline SUMMATION & 180.1 & & & & 165746.45 \\
\hline & & & & $\begin{array}{l}\text { WEIGHTED } \\
\text { AVERAGE }\end{array}$ & $\begin{array}{r}920.302332 \\
920.30 \mathrm{cP}\end{array}$ \\
\hline
\end{tabular}


9.5 APPENDIX E

TOTAL SOLAR IRRADIANCE RECORDED DURING WEATHERING PERIOD 
TOTAL SOLAR IRRADIANCE VS. WEATHERING PERIOD

\begin{tabular}{|c|c|}
\hline Weathering Period ( hours ) & Total Solar Irradiance ( W/ m2 ) \\
\hline unweathered crude oil & 0 \\
\hline 4 & 2366.6 \\
\hline 8 & 6102.1 \\
\hline 24 & $8484.86^{*}$ \\
\hline 96 & 27338.85 \\
\hline 168 & 42973.63 \\
\hline & \\
\hline 96 & 30421.23 \\
\hline
\end{tabular}

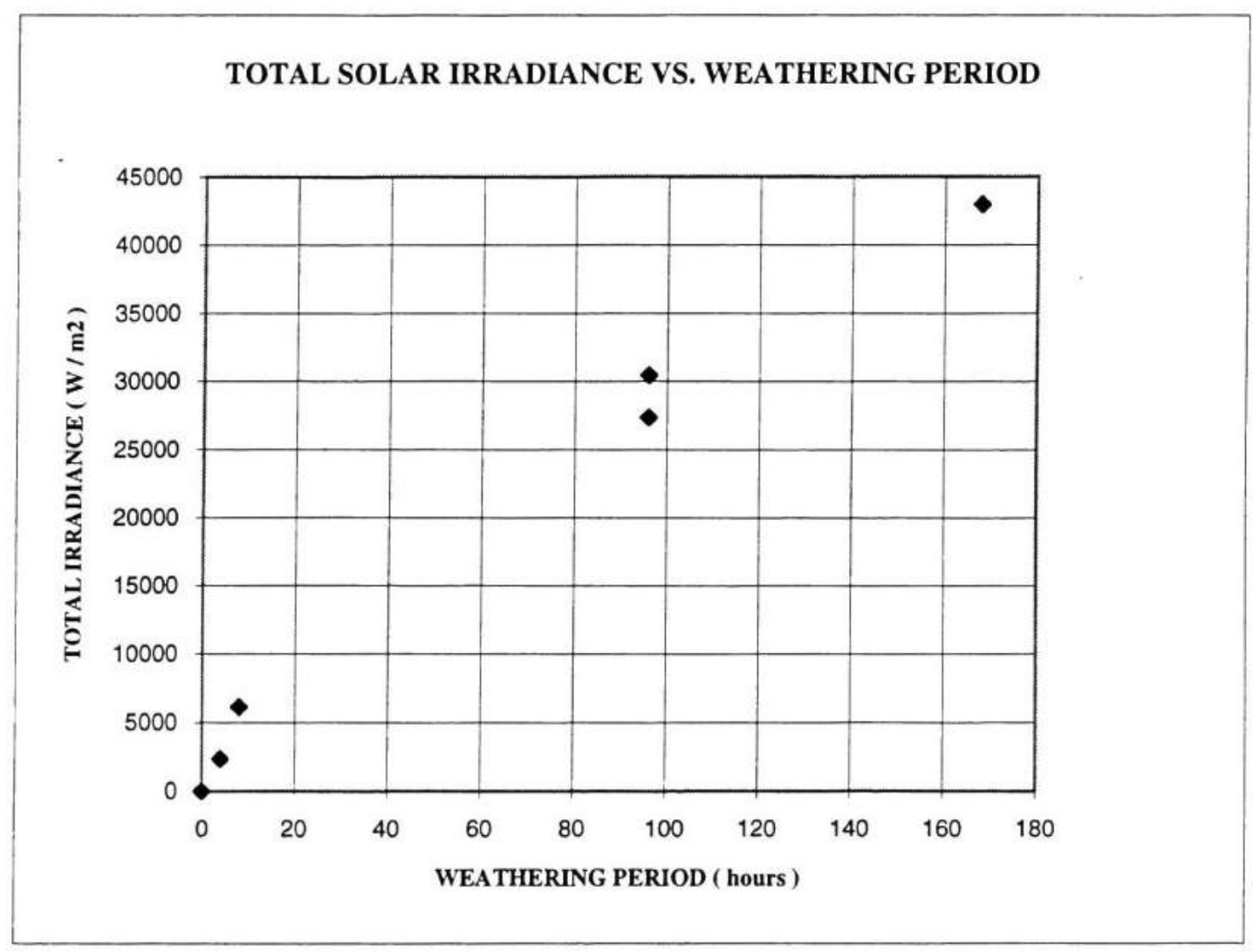

* Data unavailable for 24 hour period ( estimated by regressional analysis )

LEAST SQUARES REGRESSION: $\mathrm{Y}=2348.726+255.6722 \mathrm{X}$ 
TOTAL SOLAR IRRADIANCE MEASURED DURING EXPOSURE PERIOD ( $\mathrm{W} / \mathrm{m} 2$ )

WEATHERING DURATION 4 HOURS

AVERAGED HOURLY SOLAR IRRADIANCE RECORDED ( $\mathrm{W} / \mathrm{m} 2$ )

WEATHERING DURATION 8 HOURS

JULIAN DAY

\begin{tabular}{|c|c|c|c|c|c|c|c|c|c|}
\hline HOUR ENDING & 160 & 161 & 162 & 163 & 164 & 165 & 166 & 167 & 168 \\
\hline 600 & & & & & & & & & \\
\hline 700 & & & & & & & & & \\
\hline 800 & & & & & & & & & \\
\hline 900 & & & & & 289.2 & & & & \\
\hline 1000 & 562 & & & & 574.9 & & & & \\
\hline 1100 & 687.8 & & & & 785 & & & & \\
\hline 1200 & 600.1 & & & & 788 & & & & \\
\hline 1300 & 331 & & & & 828 & & & & \\
\hline 1400 & 185.7 & & & & 935 & & & & \\
\hline 1500 & & & & & 990 & & & & \\
\hline 1600 & & & & & 912 & & & & \\
\hline 1700 & & & & & & & & & \\
\hline 1800 & & & & & & & & & \\
\hline 1900 & & & & & & & & & \\
\hline 2000 & & & & & & & & & \\
\hline 2100 & & & & & & & & & \\
\hline
\end{tabular}

TOTAL DAY

2366.6

6102.1 
TOTAL SOLAR IRRADIANCE MEASURED DURING EXPOSURE PERIOD ( $\mathrm{W} / \mathrm{m} 2$ )

WEATHERING DURATION 4DAYS AVERAGED HOURLY SOLAR IRRADIANCE RECORDED ( W/m 2 )

JULIAN DAY

\begin{tabular}{|c|c|c|c|c|c|c|c|c|c|}
\hline HOUR ENDING & 174 & 175 & 176 & 177 & 178 & 179 & 180 & 182 & 183 \\
\hline & & & & & & & & & \\
\hline 600 & & 0 & 0 & 0 & 0 & & & & \\
\hline 700 & & 5.744 & 5.351 & 4.474 & 3.587 & & & & \\
\hline 800 & & 85.6 & 82.3 & 83.6 & 17.98 & & & & \\
\hline 900 & & 206.8 & 261.8 & 253.9 & 29.04 & & & & \\
\hline 1000 & & 327.8 & 479 & 461.8 & 190.8 & & & & \\
\hline 1100 & 669 & 557.5 & 680.1 & 610.8 & & & & & \\
\hline 1200 & 799 & 706 & 824 & 779 & & & & & \\
\hline 1300 & 919 & 792 & 919 & 875 & & & & & \\
\hline 1400 & 1027 & 875 & 943 & 882 & & & & & \\
\hline 1500 & 886 & 865 & 820 & 852 & & & & & \\
\hline 1600 & 898 & 639.9 & 613.6 & 886 & & & & & \\
\hline 1700 & 647.6 & 613 & 498.7 & 693.8 & & & & & \\
\hline 1800 & 484.7 & 480.7 & 282.9 & 528.1 & & & & & \\
\hline 1900 & 271.5 & 272.2 & 152.2 & 255 & & & & & \\
\hline 2000 & 74.3 & 79.9 & 76.6 & 98.7 & & & & & \\
\hline 2100 & 3.488 & 5.316 & 0.973 & 1.701 & & & & & \\
\hline
\end{tabular}

\begin{tabular}{|l|l|l|l|l|l|}
\hline TOTAL DAY & 6679.588 & 6512.46 & 6639.524 & 7265.875 & 241.407 \\
\hline
\end{tabular} 
TOTAL SOLAR IRRADIANCE MEASURED DURING EXPOSURE PERIOD ( $\mathrm{W} / \mathrm{m} 2$ )

WEATHERING DURATION 4 DAYS AVERAGED HOURLY SOLAR IRRADIANCE RECORDED ( W / m 2 )

JULIAN DAY

\begin{tabular}{|c|c|c|c|c|c|c|c|c|c|}
\hline HOUR ENDING & 132 & 133 & 134 & 135 & 136 & 137 & 138 & 139 & 140 \\
\hline & & & & & & & & & \\
\hline 600 & & 0 & 0 & 0 & 0 & & & & \\
\hline 700 & & 4.438 & 5.417 & 4.468 & 4.315 & & & & \\
\hline 800 & & 80.4 & 56.93 & 45.49 & 43.91 & & & & \\
\hline 900 & & 323.8 & 259 & 280.3 & 311.4 & & & & \\
\hline 1000 & & 539.3 & 471.7 & 601.1 & 577.7 & & & & \\
\hline 1100 & & 599.7 & 667.2 & 698.5 & 675.7 & & & & \\
\hline 1200 & 921 & 685.1 & 767 & 850 & 780 & & & & \\
\hline 1300 & 998 & 562.6 & 1037 & 961 & & & & & \\
\hline 1400 & 1019 & 898 & 938 & 1083 & & & & & \\
\hline 1500 & 991 & 929 & 888 & 954 & & & & & \\
\hline 1600 & 909 & 836 & 917 & 900 & & & & & \\
\hline 1700 & 598.1 & 701 & 542.2 & 742 & & & & & \\
\hline 1800 & 238.6 & 486.9 & 460.8 & 529.1 & & & & & \\
\hline 1900 & 159 & 228.5 & 212.6 & 265.6 & & & & & \\
\hline 2000 & 21.19 & 44.51 & 48.43 & 66.28 & & & & & \\
\hline 2100 & 0.399 & 0.467 & 0.538 & 0.551 & & & & & \\
\hline
\end{tabular}

\begin{tabular}{|l|r|r|r|r|r|r|}
\hline TOTAL DAY & 5855.289 & 6919.715 & 7271.815 & 7981.389 & 2393.025 \\
\hline
\end{tabular} 
TOTAL SOLAR IRRADIANCE MEASURED DURING EXPOSURE PERIOD ( $\mathbf{W} / \mathrm{m} 2$ )

WEATHERING DURATION 7 DAYS AVERAGED HOURLY SOLAR IRRADIANCE RECORDED ( W/m 2 )

\section{JULIAN DAY}

\begin{tabular}{|c|c|c|c|c|c|c|c|c|c|}
\hline HOUR ENDING & 166 & 167 & 168 & 169 & 170 & 171 & 172 & 173 & 174 \\
\hline & & & & & & & & & \\
\hline 600 & & 0 & 0 & 0 & 0 & 0 & 0 & 0 & \\
\hline 700 & & 6.116 & 6.077 & 6.61 & 6.112 & 3.353 & 3.919 & 7.19 & \\
\hline 800 & & 76.7 & 93.3 & 94.5 & 82.4 & 38.61 & 44.08 & 56.17 & \\
\hline 900 & 369 & 284.4 & 244 & 308.7 & 337.4 & 150.1 & 157.8 & 236.3 & \\
\hline 1000 & 351 & 523.1 & 487.8 & 481.2 & 555.2 & 340.4 & 465.9 & & \\
\hline 1100 & 654 & 642.9 & 727 & 569.4 & 758 & 570.7 & 588.5 & & \\
\hline 1200 & 831 & 771 & 563.8 & 855 & 712 & 653.5 & 777 & & \\
\hline 1300 & 790 & 876 & 782 & 594 & 1010 & 929 & 938 & & \\
\hline 1400 & 383.8 & 1010 & 657.8 & 724 & 717 & 1018 & 998 & & \\
\hline 1500 & 512.8 & 942 & 323.9 & 522.7 & 533.6 & 985 & 916 & & \\
\hline 1600 & 774 & 760 & 382 & 582.6 & 286.7 & 417 & 793 & & \\
\hline 1700 & 679.6 & 749 & 383.2 & 707 & 92.6 & 562.6 & 526.3 & & \\
\hline 1800 & 514.7 & 568.8 & 472.8 & 125 & 166.9 & 274.6 & 153.5 & & \\
\hline 1900 & 231.4 & 172 & 212.1 & 32.99 & 146.1 & 113.5 & 54.76 & & \\
\hline 2000 & 36.65 & 35.92 & 56.37 & 70.1 & 80.6 & 67.67 & 20.8 & & \\
\hline 2100 & 2.514 & 2.252 & 1.909 & 1.551 & 4.065 & 2.55 & 1.094 & & \\
\hline
\end{tabular}


9.6 APPENDIX F

PERCENTAGE OF CRUDE OIL MASS LOST TO EVAPORATION DURING WEATHERING PERIOD 
EVAPORATIVE MASS REDUCTION VS. WEATHERING PERIOD

\begin{tabular}{|r|r|r|r|r|r|}
\hline \multicolumn{1}{|c|}{ Weathering Period } & \multicolumn{1}{c|}{$\begin{array}{c}\text { Initial } \\
\text { Weight } \\
\text { (hours })\end{array}$} & $\begin{array}{c}\text { Oil } \\
\text { Input } \\
(\mathrm{g})\end{array}$ & $\begin{array}{c}\text { Final } \\
\text { Weight } \\
(\mathrm{g})\end{array}$ & $\begin{array}{c}\text { Weight } \\
\text { Reduction } \\
(\mathrm{g})\end{array}$ & $\begin{array}{c}\text { Percent } \\
\text { Loss } \\
\%\end{array}$ \\
\hline & & & & & 0 \\
\hline N/A & N/A & N/A & 10.8 & 4.871 \\
\hline 4 & 2056.5 & 221.7 & 2045.7 & 34.1 & 15.649 \\
\hline 2 & 2065.5 & 217.9 & 2031.4 & 33.8 & 15.399 \\
\hline 96 & 2077.7 & 219.5 & 2043.9 & 46.8 & 21.38 \\
\hline 168 & 2076.2 & 218.9 & 2029.4 & 50.8 & 23.091 \\
\hline
\end{tabular}

\section{Percent of Crude Oil Mass Lost to Evaporation versus Weathering Period}

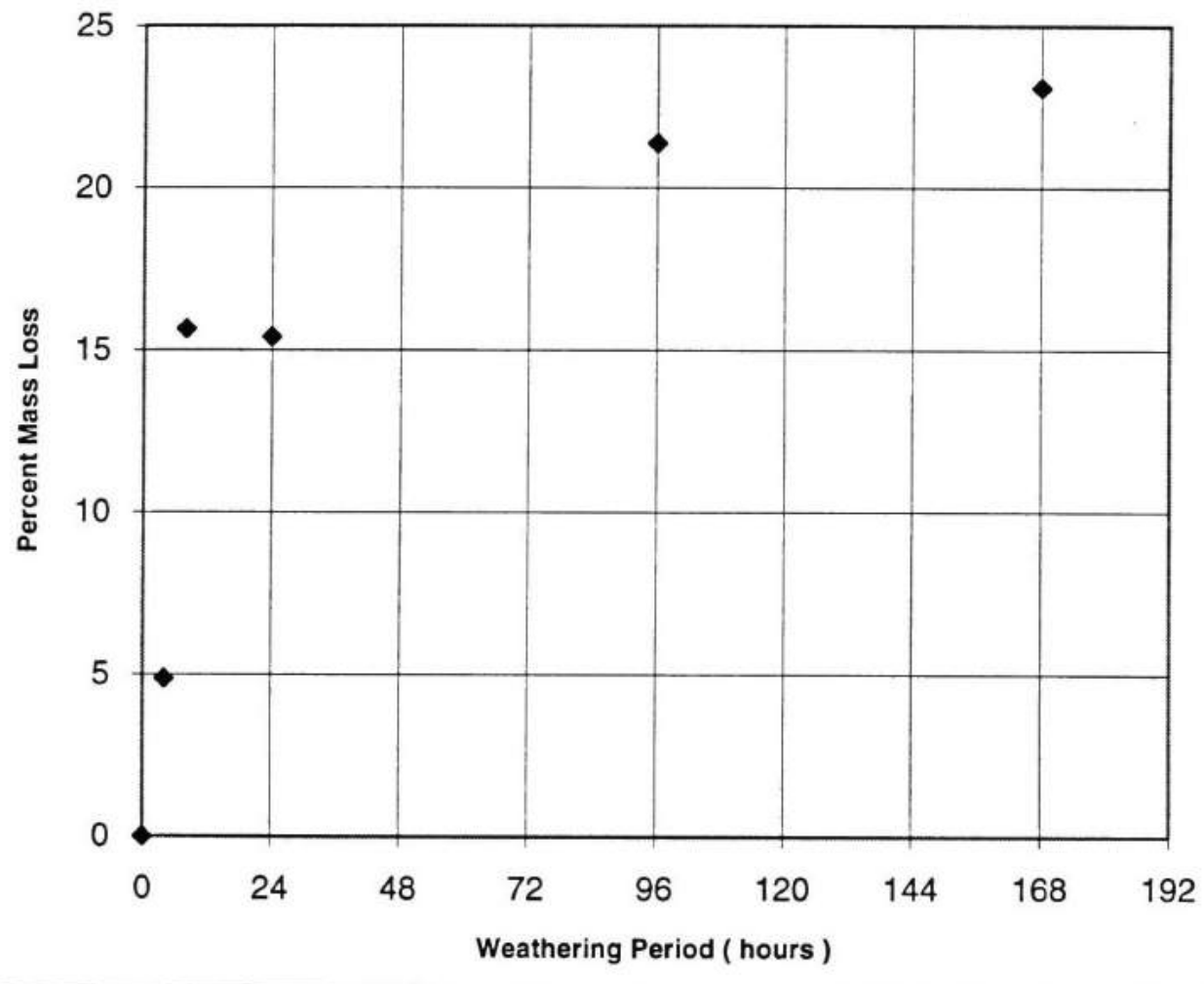


9.7 APPENDIX G

DENSITY AND SPECIFIC GRAVITY OF WEATHERED ARABIAN CRUDE OIL SAMPLES 


\begin{tabular}{|c|c|c|c|c|c|c|c|c|}
\hline $\begin{array}{c}\text { Weathering } \\
\text { Period } \\
\text { (hours) }\end{array}$ & $\begin{array}{c}\text { Initial } \\
\text { Weight } \\
(\mathrm{g})\end{array}$ & $\begin{array}{c}\text { Final } \\
\text { Weight } \\
(\mathrm{g})\end{array}$ & $\begin{array}{c}\text { delta } \\
(\mathrm{g})\end{array}$ & $\begin{array}{c}\text { Pycnometer } \\
\text { Volume } \\
(\mathrm{mL})\end{array}$ & $\begin{array}{c}\text { Oil } \\
\text { Density } \\
(\mathrm{g} / \mathrm{mL})\end{array}$ & $\begin{array}{c}\text { Pycnometer } \\
\text { Constant }\end{array}$ & $\begin{array}{c}\text { Oil Density } \\
\text { Corrected } \\
(\mathrm{g} / \mathrm{mL})\end{array}$ & $\begin{array}{c}\text { Specific } \\
\text { Gravity }\end{array}$ \\
\hline & & & & & & & & \\
\hline 0 & 14.933 & 23.8478 & 8.9148 & 10 & 0.89148 & 0.9948 & 0.8868443 & 0.889334 \\
\hline 4 & 14.933 & 24.1986 & 9.2656 & 10 & 0.92656 & 0.9948 & 0.92174189 & 0.92433 \\
\hline 8 & 14.933 & 24.285 & 9.352 & 10 & 0.9352 & 0.9948 & 0.93033696 & 0.932949 \\
\hline 24 & 14.933 & 24.2999 & 9.3669 & 10 & 0.93669 & 0.9948 & 0.93181921 & 0.934436 \\
\hline 96 & 14.9328 & 24.3986 & 9.4658 & 10 & 0.94658 & 0.9948 & 0.94165778 & 0.944302 \\
\hline 168 & 14.9328 & 24.4518 & 9.519 & 10 & 0.9519 & 0.9948 & 0.94695012 & 0.949609 \\
\hline & & & & & & & & \\
\hline 96 & 14.933 & 24.434 & 9.501 & 10 & 0.9501 & 0.9948 & 0.94515948 & 0.947813 \\
\hline
\end{tabular}

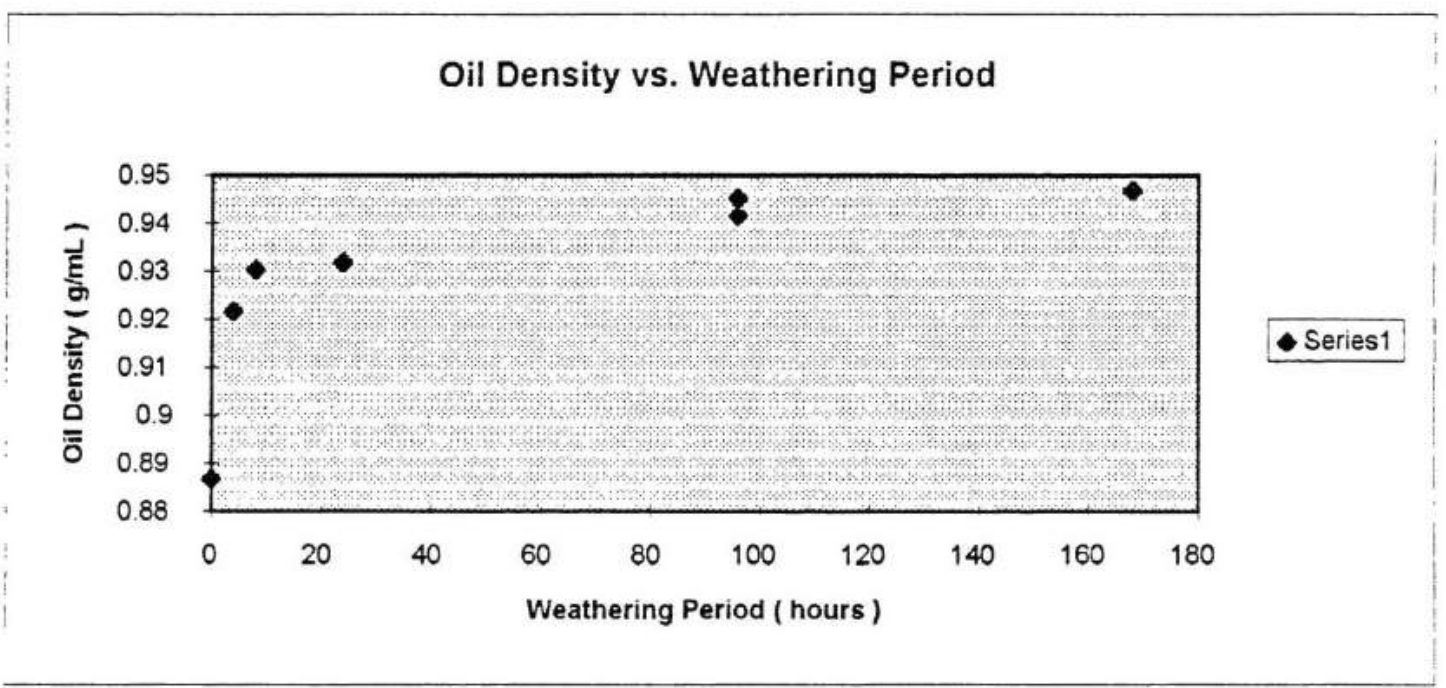

\section{Oil Specific Gravity vs. Weathering Period}

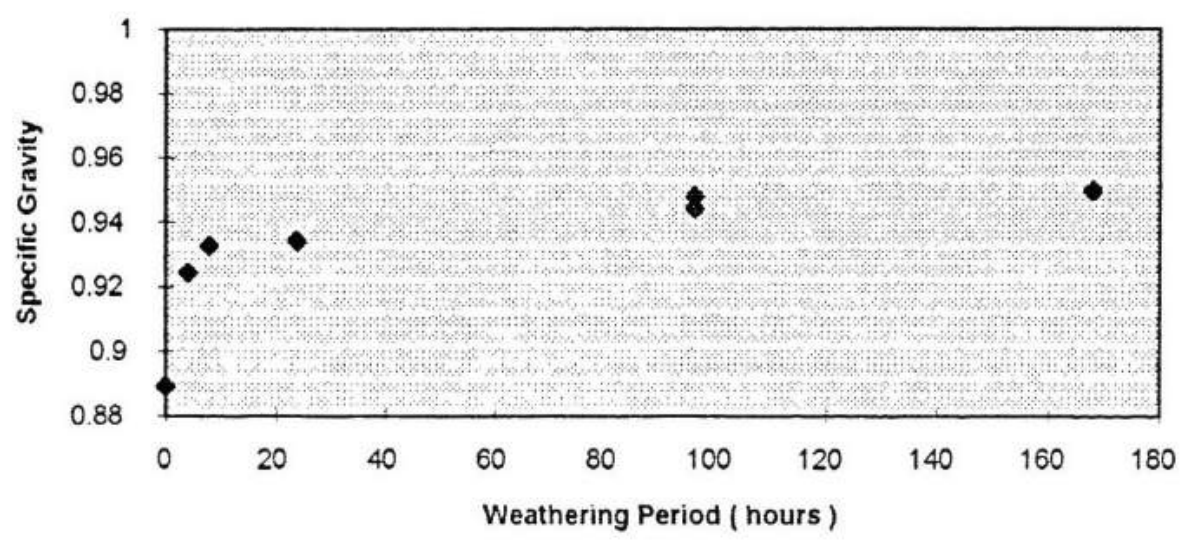

Series 1 
9.8 APPENDIX H

OIL-WATER INTERFACIAL SURFACE TENSION OF WEATHERED ARABIAN CRUDE OIL SAMPLES 
Pre-Test Calibration with Hexane-Water

\begin{tabular}{|c|c|c|c|c|c|c|}
\hline $\begin{array}{l}\text { Weathering } \\
\text { Period } \\
\text { (hours ) }\end{array}$ & $\begin{array}{c}\text { Apparent } \\
\text { Tension } \\
\text { (dynes/cm ) }\end{array}$ & $\begin{array}{l}\text { Density } \\
\text { Hexane } \\
(\mathrm{g} / \mathrm{mL})\end{array}$ & $\begin{array}{l}\text { Correction } \\
\text { Factor }\end{array}$ & $\begin{array}{c}\text { Absolute } \\
\text { Tension } \\
\text { (dynes/cm ) }\end{array}$ & $\begin{array}{c}\text { Standard } \\
\text { Value } \\
\text { (dynes/cm ) }\end{array}$ & $\begin{array}{c}\text { Calibration } \\
\text { Constant }\end{array}$ \\
\hline & & & & & & \\
\hline 0 & 46.6 & 0.6603 & 0.98971559 & 46.1207 & 51.1 & 1.108 \\
\hline 4 & 47.5 & 0.6603 & 0.99175244 & 47.1082 & 51.1 & 1.085 \\
\hline 8 & 49.6 & 0.6603 & 0.99644566 & 49.4237 & 51.1 & 1.034 \\
\hline 24 & 49.8 & 0.6603 & 0.99688841 & 49.645 & 51.1 & 1.029 \\
\hline 96 & 48.2 & 0.6603 & 0.99332597 & 47.8783 & 51.1 & 1.067 \\
\hline 168 & 49.5 & 0.6603 & 0.99622401 & 49.313 & 51.1 & 1.036 \\
\hline
\end{tabular}

\section{Oil-Water IFST Determination}

\begin{tabular}{|c|c|c|c|c|c|c|}
\hline $\begin{array}{l}\text { Weathering } \\
\text { Period } \\
\text { ( hours ) }\end{array}$ & $\begin{array}{c}\text { Apparent } \\
\text { Tension } \\
\text { ( dynes/cm ) }\end{array}$ & $\begin{array}{c}\text { Density } \\
\text { Oil } \\
(\mathrm{g} / \mathrm{mL})\end{array}$ & $\begin{array}{l}\text { Correction } \\
\text { Factor }\end{array}$ & $\begin{array}{c}\text { Absolute } \\
\text { Tension } \\
\text { (dynes } / \mathrm{cm} \text { ) }\end{array}$ & $\begin{array}{c}\text { Calibration } \\
\text { Constant }\end{array}$ & $\begin{array}{c}\text { Interfacial } \\
\text { Tension } \\
\text { (dynes/cm ) }\end{array}$ \\
\hline \multirow[t]{2}{*}{0} & 26.8 & 0.8868 & 1.0602997 & 28.41603196 & 1.108 & 31.485 \\
\hline & 28.4 & 0.8868 & 1.06894859 & 30.35813996 & 1.108 & 33.637 \\
\hline \multirow[t]{2}{*}{4} & 26.5 & 0.9217 & 1.12035009 & 29.68927739 & 1.085 & 32.213 \\
\hline & 26 & 0.9217 & 1.11694031 & 29.04044806 & 1.085 & 31.509 \\
\hline \multirow[t]{2}{*}{8} & 25.8 & 0.9303 & 1.13773491 & 29.35356068 & 1.034 & 30.351 \\
\hline & 22.6 & 0.9303 & 1.11353675 & 25.16593055 & 1.034 & 26.022 \\
\hline \multirow[t]{2}{*}{24} & 25.9 & 0.9318 & 1.14279826 & 29.59847493 & 1.029 & 30.457 \\
\hline & 24.6 & 0.9318 & 1.13304144 & 27.87281942 & 1.029 & 28.681 \\
\hline \multirow[t]{2}{*}{96} & 23.2 & 0.9416 & 1.15297883 & 26.74910886 & 1.067 & 28.541 \\
\hline & 21.6 & 0.9416 & 1.13912669 & 24.6051365 & 1.067 & 26.254 \\
\hline \multirow[t]{2}{*}{168} & 20.9 & 0.947 & 1.15253746 & 24.08803291 & 1.036 & 24.955 \\
\hline & 18.6 & 0.947 & 1.13023588 & 21.02238737 & 1.036 & 21.779 \\
\hline
\end{tabular}




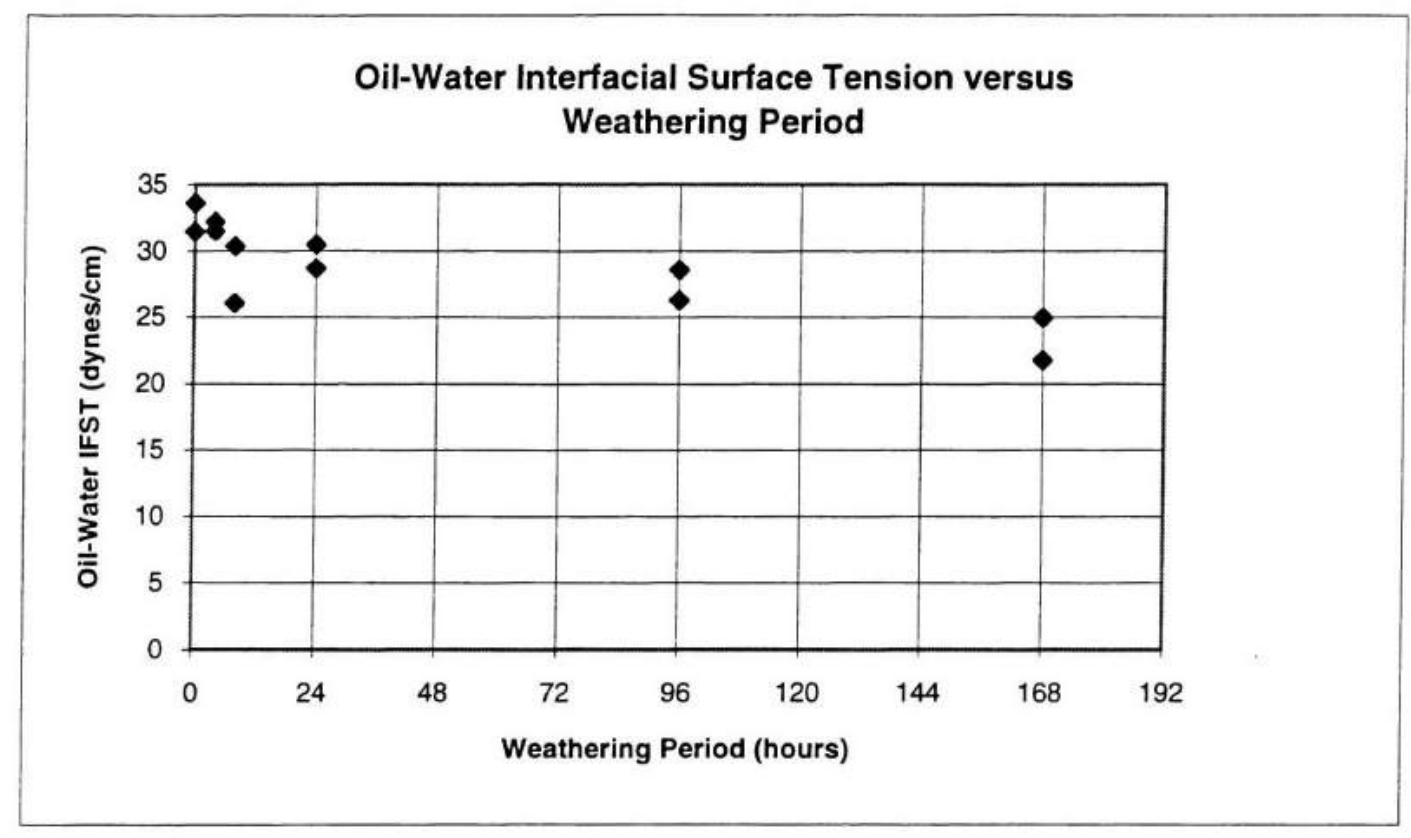


TOTAL ION CHROMATOGRAMS AND SELECTED ION CHROMATOGRAMS FOR WEATHERED ARABIAN CRUDE OIL SAMPLES, AS DETERMINED BY GAS CHROMATOGRAPHY/MASS SPECTROMETRY

- UNWEATHERED ARABIAN CRUDE OIL SAMPLE

- WEATHERING PERIODS

- 8 HOURS

- 24 HOURS

- 4 DAYS

- 7 DAYS 


\section{INDEX TO REFERENCED COMPOUND PEAKS \\ (REFER TO UNWEATHERED SAMPLE OUTPUT - FILE DJBNO.D)}

ION NUMBER

57

128

142

156

178
2

3

4

5

6

PEAK NUMBER

1

2

3

1

1

2

1

2

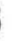

4

1
COMPOUND NAME

n-C11

$\mathrm{n}-\mathrm{C} 19$

n-C30

Naphthalene

2-Methyl-Naphthalene

1-methyl-Naphthalene

1+2 Et Naphthalene

2,6 di-methyl Naphthalene

2,7 di-methyl Naphthalene

$1,3+1,7$ di-methyl Naphthalene

1,6 di-methyl Naphthalene

2,3 + 1,4 di-methyl Naphthalene

1,5 di-methyl Naphthalene

1,2 di-methyl Naphthalene

Phenanthrene 


\section{INDEX TO REFERENCED COMPOUND PEAKS (REFER TO UNWEATHERED SAMPLE OUTPUT - FILE DJBNO.D)}

ION NUMBER

191

.

4

192

206
5

6

7

\section{PEAK NUMBER}

1

2

3

1

1

\section{COMPOUND NAME}

C29 $\alpha \beta$ Hopane

C30 $\alpha \beta$ Hopane

C31 $\alpha \beta$ S Hopane - double peak

C31 $\alpha \beta$ R Hopane

C32 $\alpha \beta$ S Hopane - double peak C32 $\alpha \beta$ R Hopane

C33 $\alpha \beta$ S Hopane - double peak C33 $\alpha \beta$ R Hopane

C34 $\alpha \beta$ S Hopane - double peak C34 $\alpha \beta$ R Hopane

C35 $\alpha \beta$ S Hopane - double peak C35 $\alpha \beta$ R Hopane

methyl-Phenanthrenes

di-methyl-Phenanthrenes 
File:

Operator:

Date Acquired:

Method File:

Sample Name:

Misc Info:

ALS vial:
C: \CHEMPC \DATA \DJBNO.D

DON. B

21 Jul 94

JAFFE.M

1
$3: 03 \mathrm{pm}$

ARABIAN CRUDE UNWEATHERED

9 X ORIGINAL

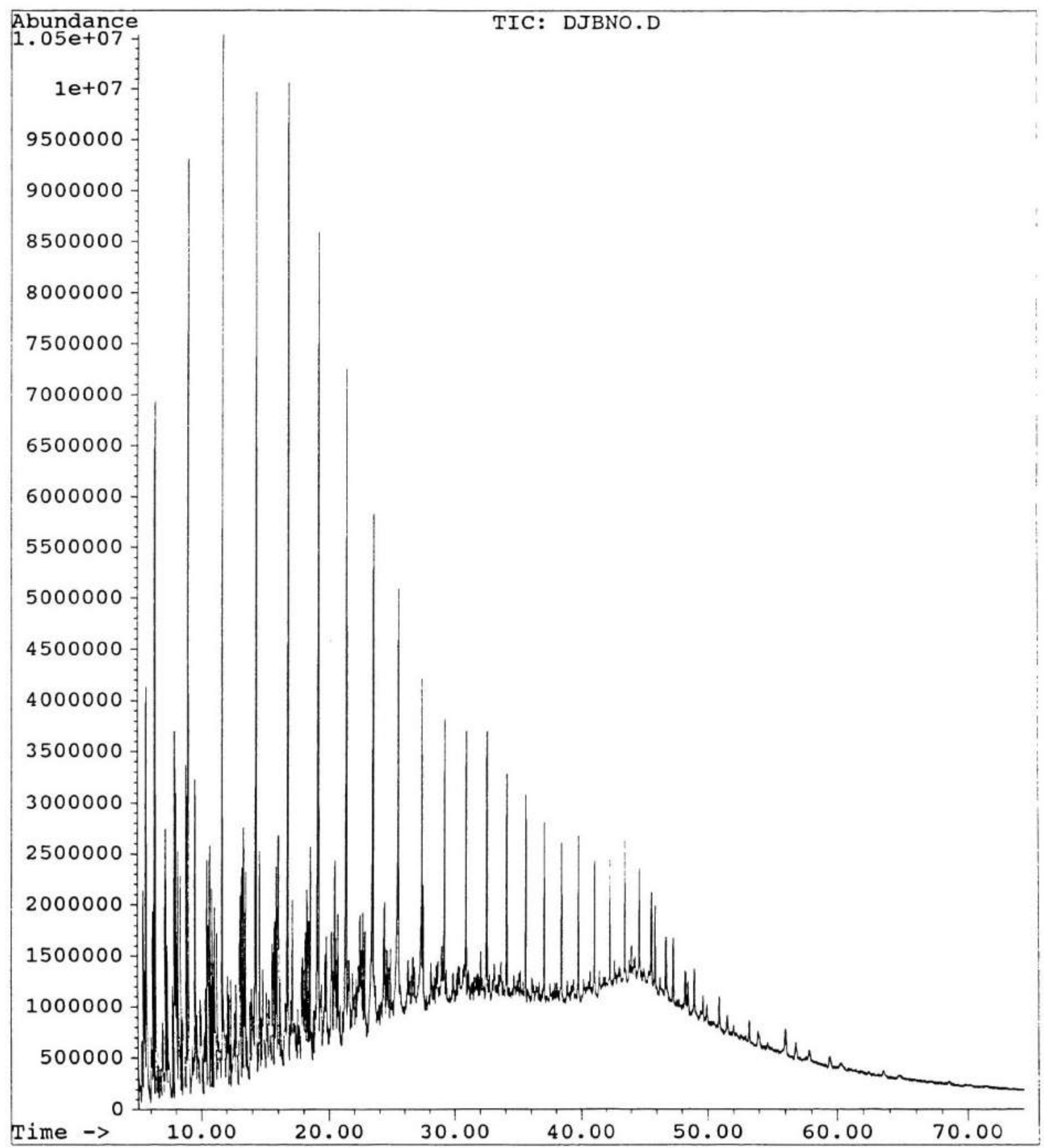


File:

C: $\backslash$ CHEMPC \DATA \DJBNO.D

Operator:

DON. B

Date Acquired: 21 Jul 94

Method File: JAFFE.M

Sample Name:

Misc Info:

ALS vial:

1

ARABIAN CRUDE UNWEATHERED

9 X ORIGINAL

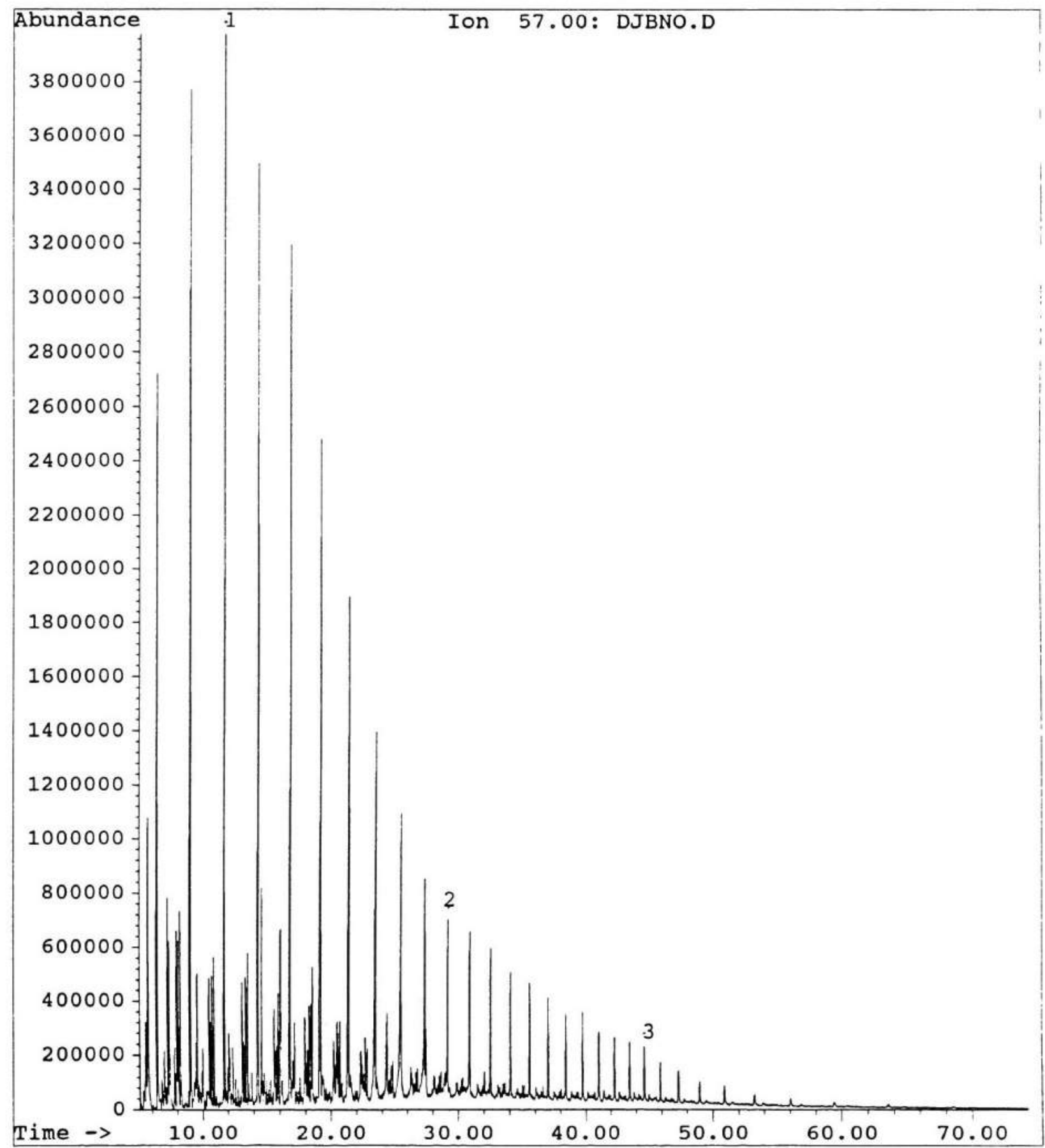


File:

Operator:

Date Acquired: 1 J I

Method File: JAFFE.M

Sample Name:

Misc Info:

ALS vial:

1
$C: \backslash C H E M P C \backslash D A T A \backslash D J B N O . D$

DON. B

3:03 pm

ARABIAN CRUDE UNWEATHERED

9 X ORIGINAL

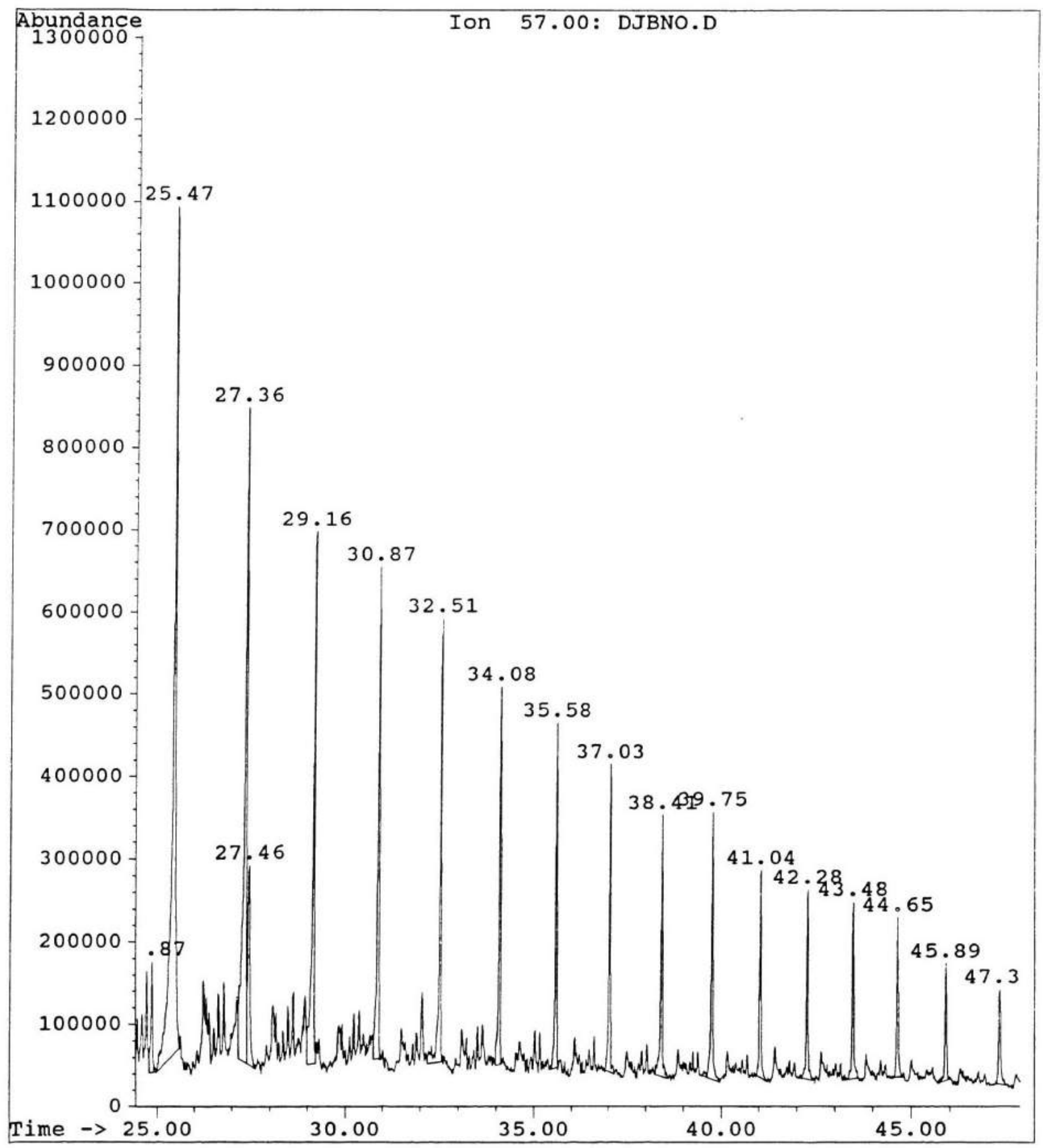


File:

Operator:

Date Acquired: 1 Jul

Method File:

Sample Name:

Misc Info:

ALS vial:
C: \CHEMPC \DATA \DJBNO.D

DON. B

21 Jul $94 \quad 3: 03 \mathrm{pm}$

JAFFE.M

ARABIAN CRUDE UNWEATHERED

9 X ORIGINAL

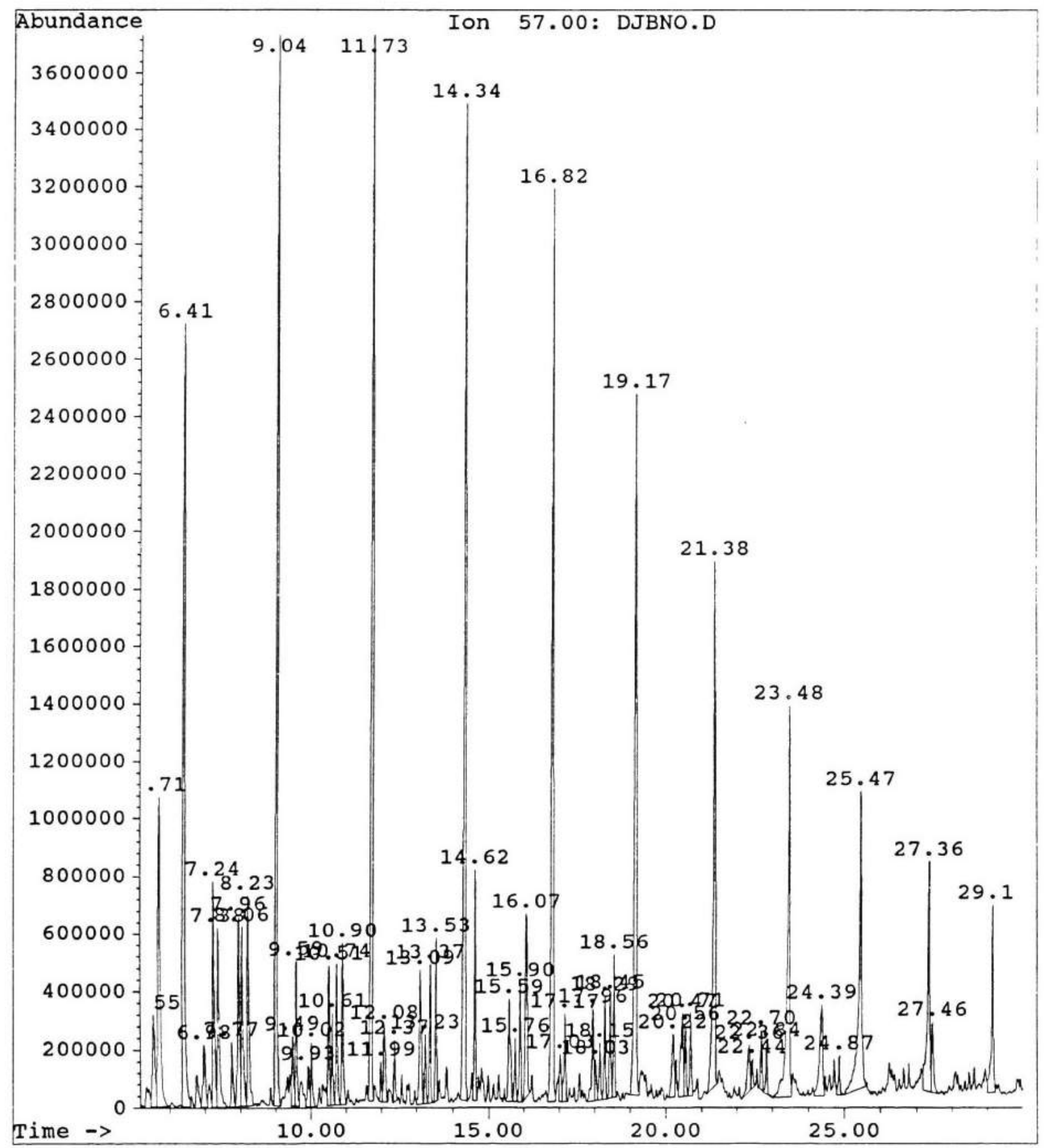


Ion 57.00: DJBNO.D

ARABIAN CRUDE UNWEATHERED

\begin{tabular}{|c|c|c|c|c|c|c|}
\hline Peak\# \# & Ret Time & Type & width & Area & Start Time & End Time \\
\hline 1 & 5.547 & VV & 0.073 & 15075979 & 5.458 & 5.612 \\
\hline 2 & 5.708 & VV & 0.069 & 48386570 & 5.612 & 5.978 \\
\hline 3 & 6.413 & PV & 0.057 & 97500416 & 6.324 & 6.576 \\
\hline 4 & 6.978 & vV & 0.066 & 9588350 & 6.880 & 7.054 \\
\hline 5 & 7.245 & vV & 0.054 & 26553756 & 7.184 & 7.324 \\
\hline 6 & 7.381 & VB & 0.058 & 23563487 & 7.324 & 7.655 \\
\hline 7 & 7.766 & BV & 0.056 & 7939502 & 7.655 & 7.853 \\
\hline 8 & 7.964 & vV & 0.055 & 23848731 & 7.853 & 8.015 \\
\hline 9 & 8.059 & vV & 0.051 & 20976960 & 8.015 & 8.166 \\
\hline 10 & 8.226 & vV & 0.052 & 23800202 & 8.166 & 8.383 \\
\hline 11 & 9.036 & VV & 0.052 & 118452450 & 8.927 & $9.13^{\circ}$ \\
\hline 12 & 9.487 & VV & 0.054 & 8668229 & 9.439 & 9.54 \\
\hline 13 & 9.588 & VV & 0.044 & 14356037 & 9.540 & 9.656 \\
\hline 14 & 9.935 & vV & 0.061 & 5379326 & 9.850 & 9.982 \\
\hline 15 & 10.024 & vV & 0.048 & 6750258 & 9.982 & 10.15 \\
\hline 16 & 10.513 & VV & 0.045 & 13614974 & 10.460 & 10.566 \\
\hline 17 & 10.613 & VV & 0.045 & 8575962 & 10.566 & 10.672 \\
\hline 18 & 10.735 & vV & 0.049 & 14439242 & 10.672 & 10.832 \\
\hline 19 & 10.900 & VV & 0.047 & 17066874 & 10.832 & 11.00 \\
\hline 20 & 11.727 & VV & 0.048 & 120933750 & 11.622 & 11.78 \\
\hline 21 & 11.987 & VV & 0.047 & 3874964 & 11.929 & 12.032 \\
\hline 22 & 12.079 & VV & 0.061 & 10441417 & 12.032 & 12.17 \\
\hline 23 & 12.371 & PV & 0.044 & 5725722 & 12.321 & 12.45 \\
\hline 24 & 13.090 & VV & 0.063 & 19399637 & 12.987 & 13.17 \\
\hline 25 & 13.235 & VV & 0.042 & 6412792 & 13.177 & 13.29 \\
\hline 26 & 13.373 & VV & 0.043 & 13350315 & 13.297 & 13.47 \\
\hline 27 & 13.533 & PV & 0.041 & 14140551 & 13.472 & 13.58 \\
\hline 28 & 14.339 & VV & 0.051 & 114841286 & 14.199 & 14.41 \\
\hline 29 & 14.621 & VV & 0.041 & 20583392 & 14.561 & 14.67 \\
\hline 30 & 15.586 & VV & 0.063 & 15378089 & 15.490 & 15.687 \\
\hline 31 & 15.761 & VV & 0.046 & 6409545 & 15.687 & 15.81 \\
\hline 32 & 15.900 & VV & 0.048 & 11960414 & 15.812 & 15.97 \\
\hline 33 & 16.072 & PV & 0.066 & 25339623 & 15.971 & 16.17 \\
\hline 34 & 16.819 & VV & 0.052 & 106686979 & 16.665 & 16.89 \\
\hline 35 & 17.030 & vV & 0.053 & 5524586 & 16.991 & 17.08 \\
\hline 36 & 17.169 & vV & 0.051 & 10158662 & 17.081 & 17.23 \\
\hline 37 & 17.956 & PV & 0.055 & 11938886 & 17.778 & 17.99 \\
\hline 38 & 18.027 & VV & 0.050 & 4523169 & 17.997 & 18.08 \\
\hline 39 & 18.151 & VV & 0.047 & 6085014 & 18.085 & 18.20 \\
\hline 40 & 18.293 & VV & 0.046 & 10260024 & 18.208 & 18.35 \\
\hline 41 & 18.446 & PV & 0.047 & 10933279 & 18.352 & 18.49 \\
\hline 42 & 18.556 & VV & 0.045 & 13313215 & 18.493 & 18.61 \\
\hline 43 & 19.168 & BV & 0.055 & 91094752 & 18.973 & 19.25 \\
\hline 44 & 20.216 & PV & 0.075 & 10276881 & 20.075 & 20.25 \\
\hline 45 & 20.474 & vV & 0.069 & 13937987 & 20.349 & 20.51 \\
\hline 46 & 20.559 & vV & 0.046 & 6921685 & 20.514 & 20.61 \\
\hline 47 & 20.706 & VV & 0.045 & 8108983 & 20.619 & 20.75 \\
\hline 48 & 21.383 & PV & 0.055 & 69947566 & 21.139 & 21.44 \\
\hline 49 & 22.361 & PV & 0.075 & 8800797 & 22.143 & $22.4 \mathrm{C}$ \\
\hline 50 & 22.440 & vV & 0.045 & 2870916 & 22.403 & 22.52 \\
\hline 51 & 22.698 & BV & 0.044 & 5595751 & 22.634 & 22.75 \\
\hline 52 & 22.844 & PB & 0.046 & 5221281 & 22.752 & 22.98 \\
\hline 53 & 23.479 & BV & 0.063 & 62429098 & 22.988 & 23.53 \\
\hline 54 & 24.391 & VV & 0.085 & 18453530 & 24.177 & 24.44 \\
\hline 55 & 24.872 & VV & 0.049 & 4474802 & 24.785 & 24.98 \\
\hline 56 & 25.467 & PV & 0.070 & 54064464 & 24.982 & 25.61 \\
\hline
\end{tabular}




$\begin{array}{llllr}57 & 27.355 & \text { VV } & 0.059 & 33921607 \\ 58 & 27.456 & \text { VV } & 0.063 & 10004333 \\ 59 & 29.155 & \text { VV } & 0.058 & 22929922 \\ 60 & 30.872 & \text { VV } & 0.047 & 17475344 \\ 61 & 32.512 & \text { VV } & 0.048 & 16539060 \\ 62 & 34.082 & \text { VV } & 0.043 & 13096075 \\ 63 & 35.584 & \text { PV } & 0.044 & 11155228 \\ 64 & 37.029 & \text { BV } & 0.045 & 10782926 \\ 65 & 38.415 & \text { VV } & 0.049 & 10076752 \\ 66 & 39.750 & \text { BV } & 0.048 & 9890311 \\ 67 & 41.039 & \text { BV } & 0.045 & 7395275 \\ 68 & 42.281 & \text { BB } & 0.049 & 7228837 \\ 69 & 43.481 & \text { PB } & 0.048 & 6512714 \\ 70 & 44.645 & \text { BB } & 0.045 & 5505069 \\ 71 & 45.894 & \text { BV } & 0.053 & 4879237 \\ 72 & 47.313 & \text { BB } & 0.060 & 4446287\end{array}$

$\begin{array}{ll}27.173 & 27.401 \\ 27.401 & 27.617 \\ 28.984 & 29.213 \\ 30.749 & 30.925 \\ 32.178 & 32.566 \\ 33.968 & 34.136 \\ 35.401 & 35.636 \\ 36.884 & 37.162 \\ 38.219 & 38.593 \\ 39.481 & 39.961 \\ 40.909 & 41.235 \\ 42.050 & 42.420 \\ 43.212 & 43.631 \\ 44.436 & 44.771 \\ 45.701 & 45.989 \\ 47.234 & 47.503\end{array}$


File:

Operator:

Date Acquired:

Method File:

Sample Name:

Misc Info:

ALS vial:
$C: \backslash$ CHEMPC $\backslash$ DATA $\backslash D J B N O . D$

DON. B

21 Jul $94 \quad 3: 03 \mathrm{pm}$

JAFFE.M

1
ARABIAN CRUDE UNWEATHERED

9 X ORIGINAL

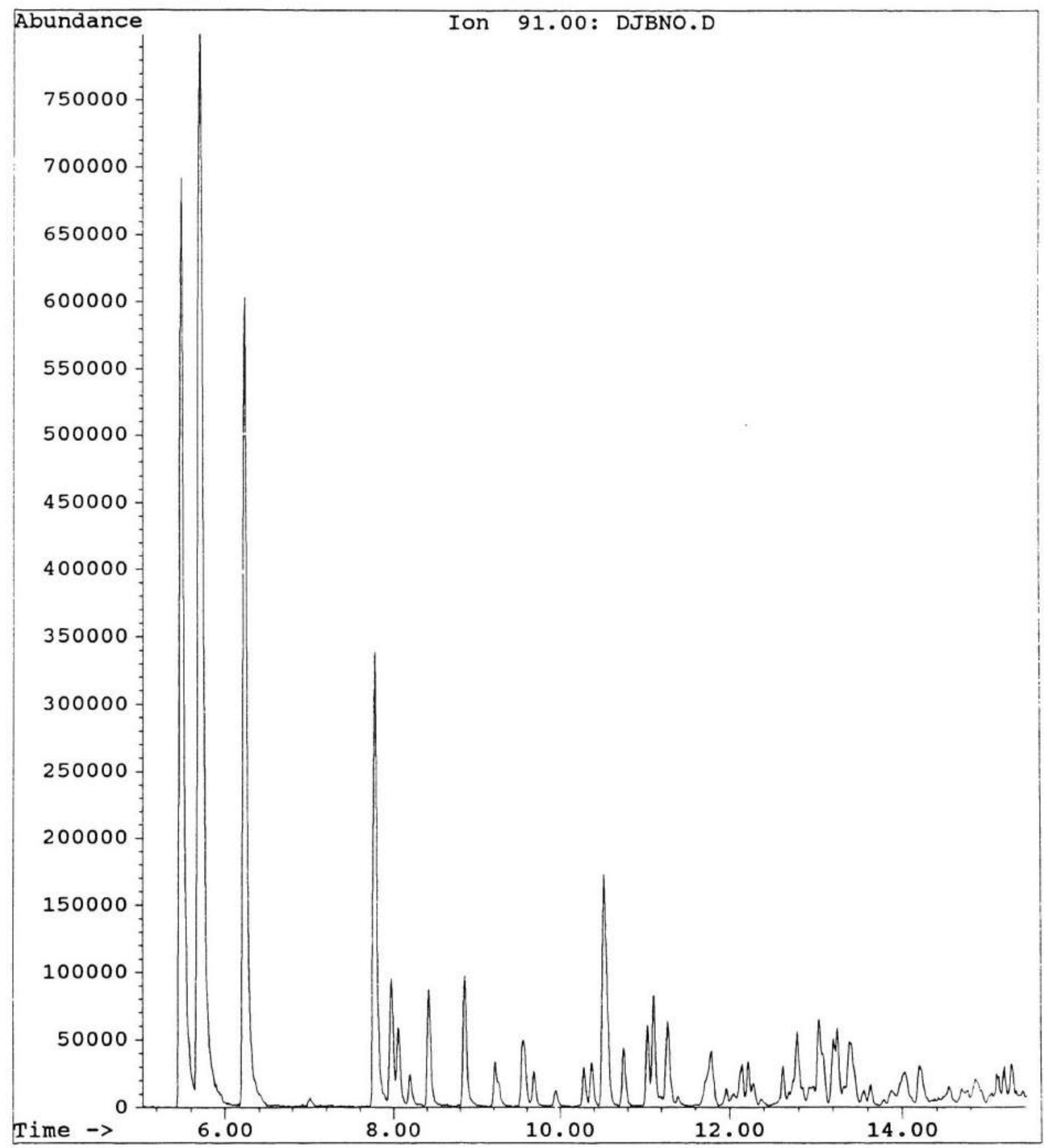


File:

Operator:

Date Acquired:

Method File:

Sample Name:

Misc Info:

ALS vial:
C: $\backslash$ CHEMPC $\backslash$ DATA $\backslash D J B N O . D$

DON. B

21 Jul 94 3:03 pm

JAFFE.M

ARABIAN CRUDE UNWEATHERED

9 X ORIGINAL

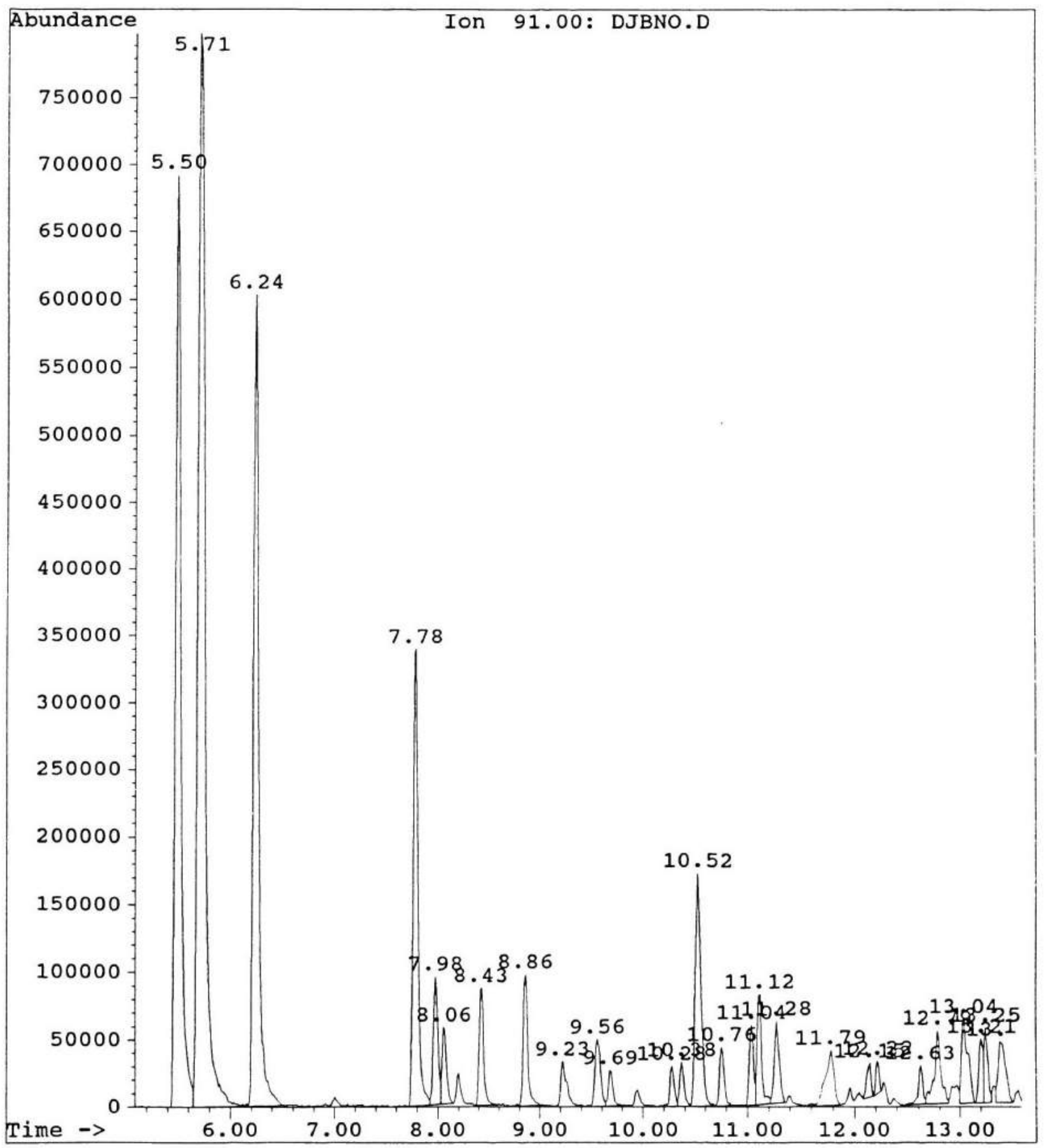


Ion 91.00: DJBNO.D

ARABIAN CRUDE UNWEATHERED

$\begin{array}{crlrrrr}\text { Peak\# } & \text { Ret Time } & \text { Type } & \text { Width } & \text { Area } & \text { Start Time } & \text { End Time } \\ 1 & 5.495 & \text { BV } & 0.059 & 25658692 & 5.432 & 5.644 \\ 2 & 5.714 & \text { VV } & 0.075 & 38552454 & 5.644 & 6.103 \\ 3 & 6.237 & \text { VV } & 0.054 & 21131820 & 6.103 & 6.543 \\ 4 & 7.781 & \text { BV } & 0.050 & 11043928 & 7.559 & 7.922 \\ 5 & 7.980 & \text { VV } & 0.051 & 2879950 & 7.922 & 8.027 \\ 6 & 8.063 & \text { VV } & 0.048 & 1739851 & 8.027 & 8.151 \\ 7 & 8.426 & \text { BB } & 0.045 & 2582526 & 8.364 & 8.585 \\ 8 & 8.859 & \text { BV } & 0.050 & 3049214 & 8.748 & 9.045 \\ 9 & 9.226 & \text { BV } & 0.058 & 1402208 & 9.150 & 9.438 \\ 10 & 9.563 & \text { PV } & 0.061 & 1902571 & 9.438 & 9.639 \\ 11 & 9.690 & \text { VB } & 0.045 & 766243 & 9.639 & 9.811 \\ 12 & 10.282 & \text { BV } & 0.045 & 851196 & 10.137 & 10.333 \\ 13 & 10.378 & \text { VV } & 0.045 & 961411 & 10.333 & 10.458 \\ 14 & 10.524 & \text { VV } & 0.064 & 6815075 & 10.458 & 10.693 \\ 15 & 10.762 & \text { VB } & 0.047 & 1283044 & 10.693 & 10.894 \\ 16 & 11.044 & \text { BV } & 0.043 & 1536711 & 11.078 & 11.078 \\ 17 & 11.117 & \text { VV } & 0.045 & 2425560 & 11.218 & 11.364 \\ 18 & 11.280 & \text { VV } & 0.052 & 2010984 & 11.565 & 11.876 \\ 19 & 11.789 & \text { BV } & 0.078 & 2266349 & 12.080 & 12.184 \\ 20 & 12.148 & \text { VV } & 0.047 & 684965 & 12.184 & 12.259 \\ 21 & 12.224 & \text { PV } & 0.037 & 513071 & 12.495 & 12.670 \\ 22 & 12.627 & \text { BV } & 0.044 & 801826 & 12.670 & 12.891 \\ 23 & 12.792 & \text { VV } & 0.064 & 2428560 & 12.995 & 13.149 \\ 24 & 13.043 & \text { VV } & 0.063 & 2795374 & 13.149 & 13.230 \\ 25 & 13.210 & \text { VV } & 0.045 & 1253784 & 13.230 & 13.301 \\ 26 & 13.252 & \text { VV } & 0.044 & 1411414 & 13.345 & 13.509 \\ 27 & 13.399 & \text { VV } & 0.075 & 2280753 & 14.149 & 14.321 \\ 28 & 14.210 & \text { VV } & 0.074 & 1318906 & 18.214 & 18.364 \\ 29 & 15.819 & \text { PV } & 0.044 & 1002756 & 715469 & \end{array}$


File:

operator:

Date Acquired: 21 Jul 9

Method File:

Sample Name:

Misc Info:

ALS vial:
$C: \backslash$ CHEMPC \DATA \DJBNO.D

DON. B

JAFFE.M

1
$3: 03 \mathrm{pm}$

ARABIAN CRUDE UNWEATHERED

9 X ORIGINAL

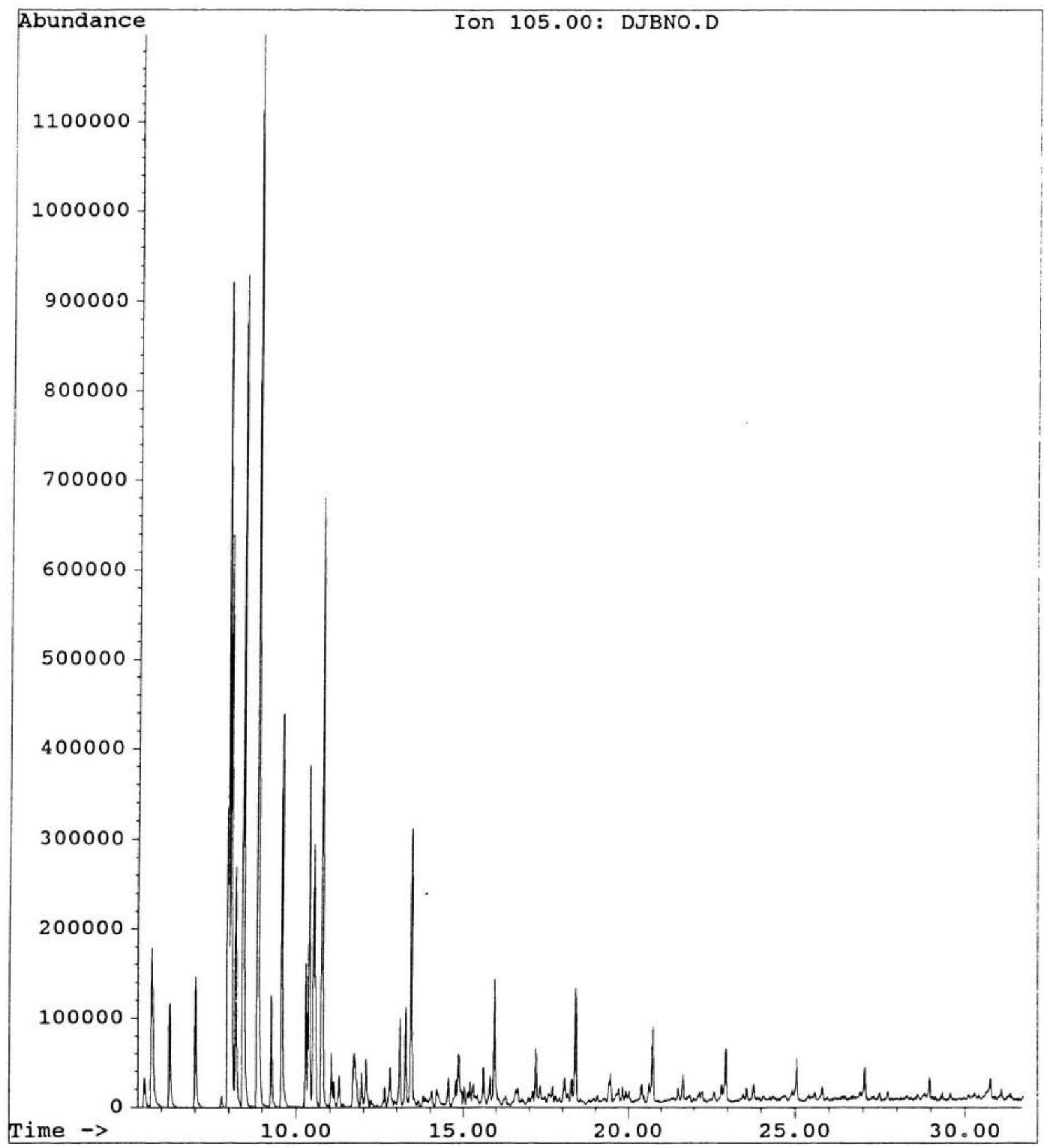


File:

Operator:

Date Acquired:

Method File:

Sample Name:

Misc Info:

ALS vial:
C: $\backslash$ CHEMPC \DATA \DJBNO.D

DON. B

21 Jul 94 3:03 pm

JAFFE.M

1

\section{$9 \mathrm{X}$ ORIGINAL}

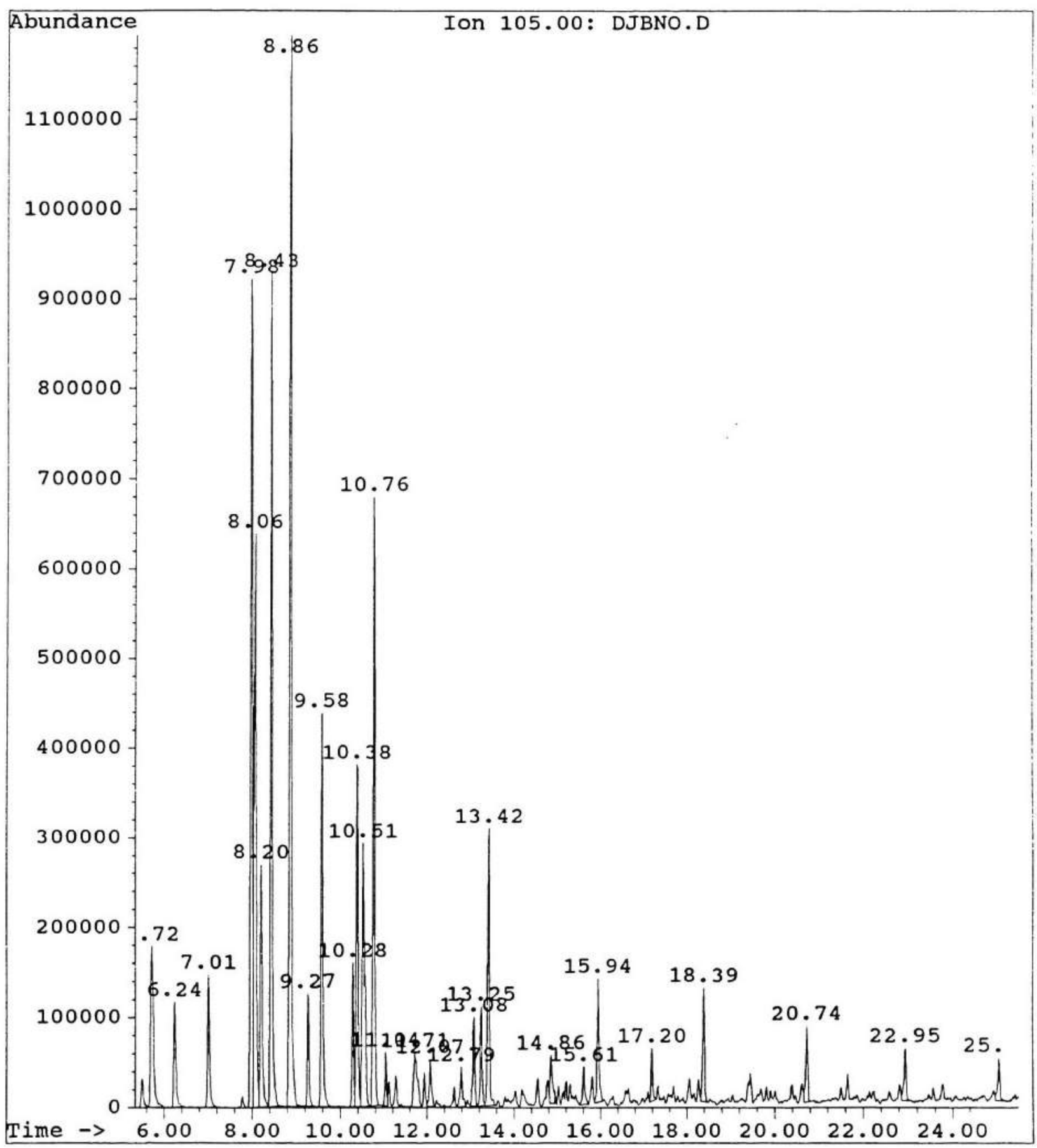


Ion 105.00: DJBNO.D

ARABIAN CRUDE UNWEATHERED

$\begin{array}{crlrr}\text { Peak\# } & \text { Ret Time } & \text { Type } & \text { Width } & \text { Area } \\ 1 & 5.716 & \text { VB } & 0.076 & 8379627 \\ 2 & 6.237 & \text { BB } & 0.053 & 3977255 \\ 3 & 7.007 & \text { BB } & 0.051 & 4589278 \\ 4 & 7.981 & \text { BV } & 0.049 & 27923151 \\ 5 & 8.064 & \text { VV } & 0.049 & 20846877 \\ 6 & 8.202 & \text { VV } & 0.050 & 8751221 \\ 7 & 8.429 & \text { VB } & 0.045 & 26740532 \\ 8 & 8.860 & \text { BB } & 0.049 & 36336030 \\ 9 & 9.269 & \text { BB } & 0.042 & 3334606 \\ 10 & 9.577 & \text { BB } & 0.048 & 13609137 \\ 11 & 10.285 & \text { BV } & 0.041 & 3974595 \\ 12 & 10.379 & \text { VV } & 0.045 & 11114646 \\ 13 & 10.515 & \text { VV } & 0.059 & 12164410 \\ 14 & 10.762 & \text { PB } & 0.045 & 18976972 \\ 15 & 11.044 & \text { BV } & 0.041 & 1489648 \\ 16 & 11.714 & \text { PV } & 0.089 & 3847221 \\ 17 & 12.070 & \text { VV } & 0.054 & 1939824 \\ 18 & 12.793 & \text { VV } & 0.055 & 1683786 \\ 19 & 13.084 & \text { VV } & 0.051 & 3092496 \\ 20 & 13.254 & \text { VV } & 0.049 & 3340789 \\ 21 & 13.420 & \text { VB } & 0.047 & 9383707 \\ 22 & 14.858 & \text { VV } & 0.060 & 2116174 \\ 23 & 15.613 & \text { PV } & 0.050 & 1453963 \\ 24 & 15.944 & \text { VV } & 0.047 & 4235260 \\ 25 & 17.199 & \text { PV } & 0.042 & 1515906 \\ 26 & 18.395 & \text { VB } & 0.048 & 3769352 \\ 27 & 20.737 & \text { VB } & 0.049 & 2673957 \\ 28 & 22.949 & \text { VV } & 0.053 & 2048063 \\ 29 & 25.057 & \text { VB } & 0.048 & 1527330\end{array}$

Start Time

5.633

6.179

6.946

7.904

8.026

8.154

8.361

8.776

9.208

9.495

10.223

10.329

10.459

10.693

10.981

11.460

11.999

12.681

12.992

13.160

13.328

14.811

15.505

15.859

17.141

18.318

20.669

22.859

24.969
End Time

5.988

6.448

7.205

8.026

8.154

8.361

8.626

9.131

9.409

9.802

10.329

10.459

10.693

10.923

11.085

11.881

12.184

12.894

13.160

13.328

13.587

14.930

15.717

16.059

17.286

18.494

20.832

23.043

25.145 
File:

Operator:

Date Acquired:

Method File:

Sample Name:

Misc Info:

ALS vial:
C: \CHEMPC \DATA \DJBNO.D

DON. B

21 Jul $94 \quad 3: 03 \mathrm{pm}$

JAFFE.M

1

\section{$9 \mathrm{X}$ ORIGINAL}

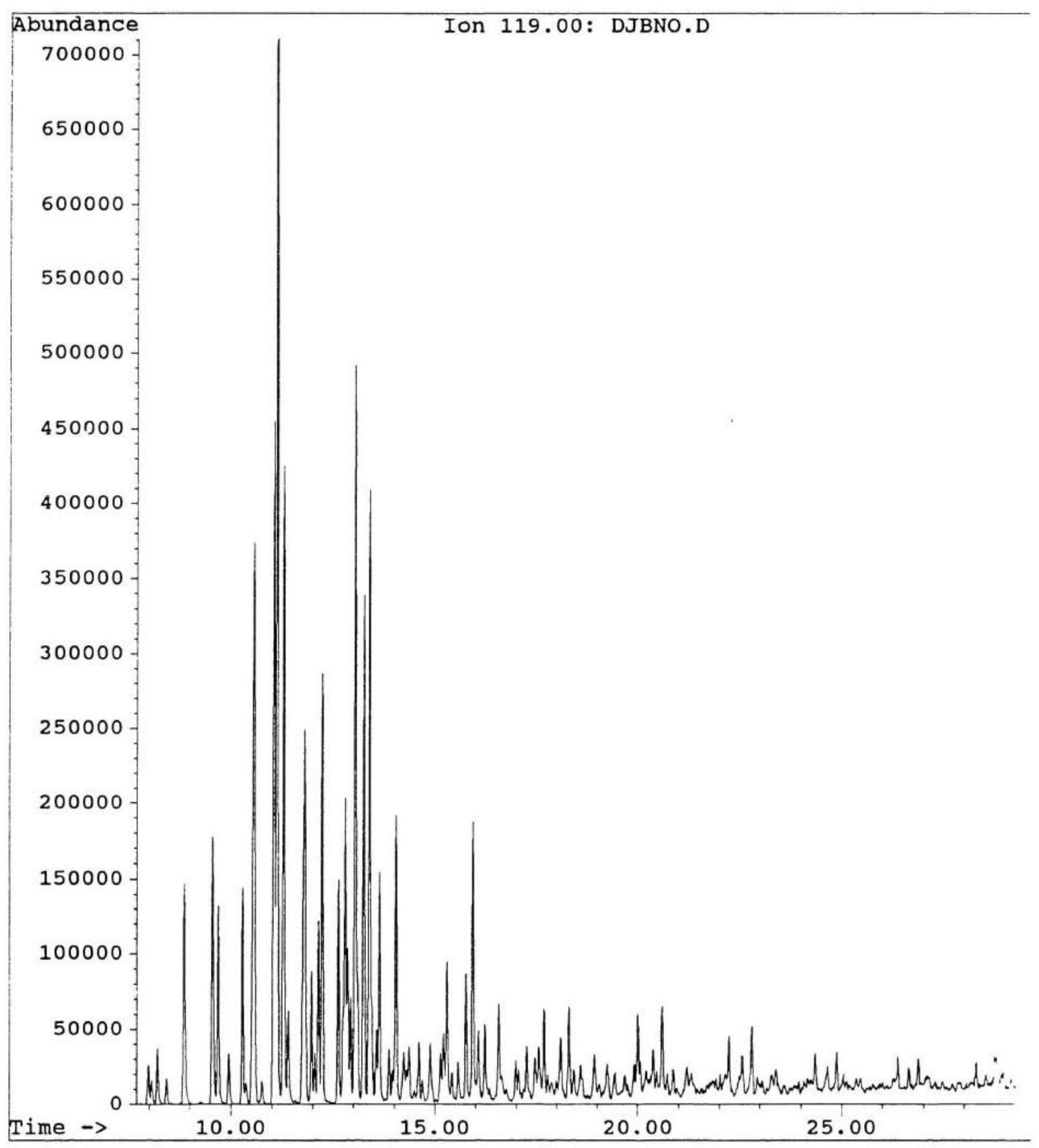


File:

operator:

Date Acquired

Method File:

Sample Name:

Misc Info:

ALS vial:
$C: \backslash$ CHEMPC \DATA \DJBNO.D

DON. B

21 Jul $94 \quad 3: 03 \mathrm{pm}$

JAFFE.M

ARABIAN CRUDE UNWEATHERED

9 X ORIGINAL

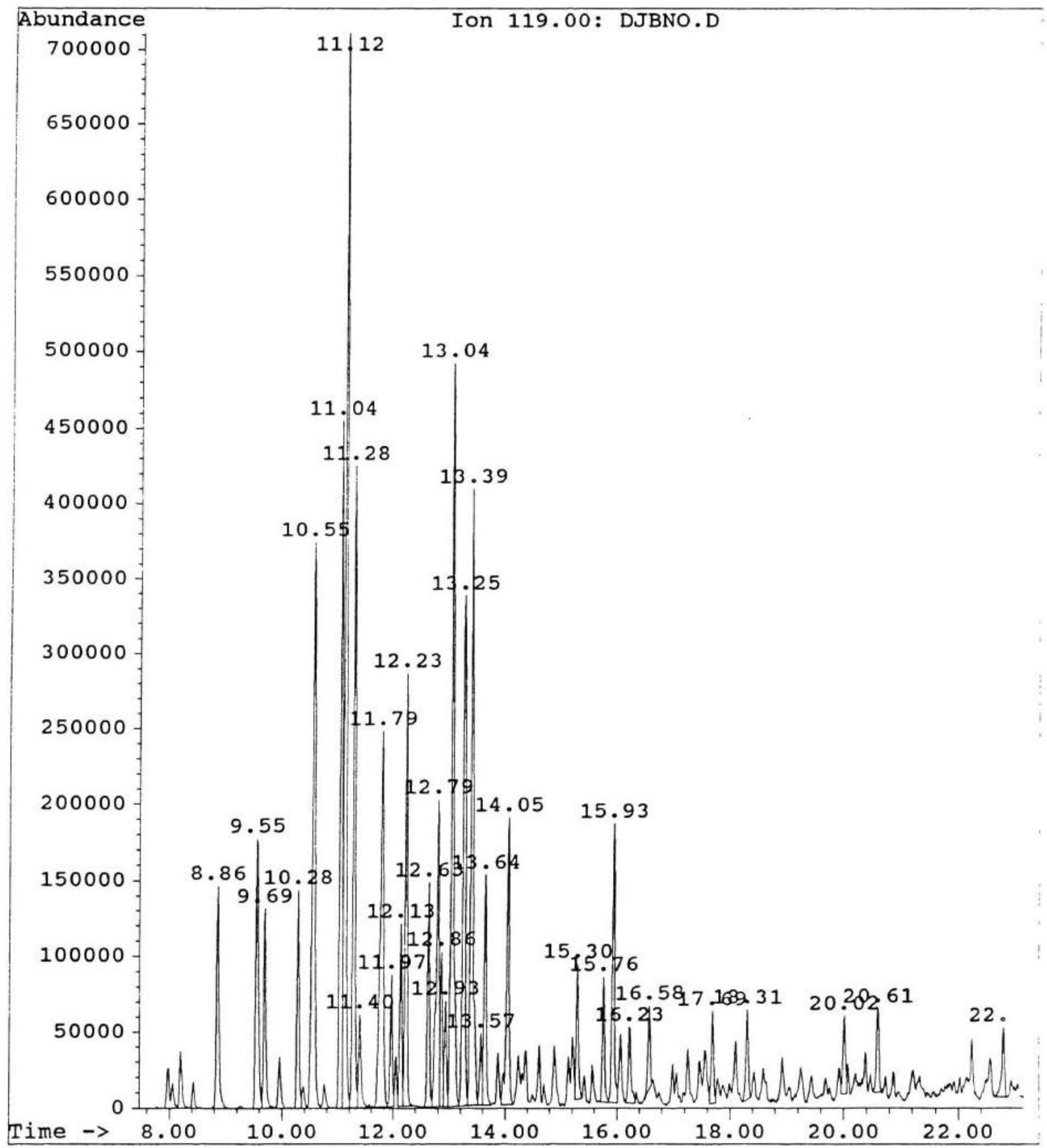


Ion 119.00: DJBNO.D

ARABIAN CRUDE UNWEATHERED

$\begin{array}{crlrrrr}\text { Peak\# } & \text { Ret Time } & \text { Type } & \text { Width } & \text { Area } & \text { Start Time } & \text { End Time } \\ 1 & 8.861 & \text { BB } & 0.051 & 4560365 & 8.738 & 9.064 \\ 2 & 9.554 & \text { BV } & 0.050 & 5798508 & 9.486 & 9.641 \\ 3 & 9.692 & \text { VB } & 0.044 & 3720925 & 9.641 & 9.850 \\ 4 & 10.284 & \text { BV } & 0.044 & 3804080 & 10.214 & 10.343 \\ 5 & 10.555 & \text { VV } & 0.056 & 13552194 & 10.453 & 10.693 \\ 6 & 11.043 & \text { BV } & 0.043 & 11803408 & 10.961 & 11.077 \\ 7 & 11.117 & \text { VV } & 0.044 & 20444244 & 11.077 & 11.216 \\ 8 & 11.280 & \text { VV } & 0.046 & 12016351 & 11.216 & 11.355 \\ 9 & 11.398 & \text { VV } & 0.047 & 1921602 & 11.355 & 11.536 \\ 10 & 11.792 & \text { VV } & 0.065 & 10668638 & 11.536 & 11.894 \\ 11 & 11.968 & \text { VV } & 0.039 & 2144751 & 11.894 & 12.013 \\ 12 & 12.134 & \text { VV } & 0.040 & 3052900 & 12.086 & 12.177 \\ 13 & 12.225 & \text { VB } & 0.042 & 7499142 & 12.177 & 12.370 \\ 14 & 12.627 & \text { BV } & 0.042 & 3913002 & 12.562 & 12.692 \\ 15 & 12.793 & \text { VV } & 0.050 & 6894519 & 12.692 & 12.831 \\ 16 & 12.855 & \text { VV } & 0.041 & 2696530 & 12.831 & 12.897 \\ 17 & 12.933 & \text { VV } & 0.040 & 1815928 & 12.897 & 12.980 \\ 18 & 13.041 & \text { VV } & 0.048 & 15244646 & 12.980 & 13.176 \\ 19 & 13.252 & \text { VV } & 0.048 & 10085709 & 13.176 & 13.324 \\ 20 & 13.389 & \text { VV } & 0.051 & 13275642 & 13.324 & 13.512 \\ 21 & 13.569 & \text { VV } & 0.042 & 1270661 & 13.512 & 13.596 \\ 22 & 13.640 & \text { VV } & 0.046 & 4209429 & 13.596 & 13.748 \\ 23 & 14.048 & \text { VV } & 0.050 & 6113374 & 13.924 & 14.147 \\ 24 & 15.297 & \text { VV } & 0.044 & 2440834 & 15.251 & 15.377 \\ 25 & 15.763 & \text { BV } & 0.044 & 2242469 & 15.705 & 15.850 \\ 26 & 15.934 & \text { VV } & 0.047 & 5399477 & 15.850 & 16.014 \\ 27 & 16.226 & \text { VV } & 0.052 & 1754394 & 16.154 & 16.328 \\ 28 & 16.578 & \text { PV } & 0.041 & 1381600 & 16.449 & 16.628 \\ 29 & 17.694 & \text { VV } & 0.046 & 1808339 & 17.633 & 17.745 \\ 30 & 18.310 & \text { PV } & 0.046 & 1773819 & 19.955 & 18.369 \\ 31 & 20.016 & \text { VV } & 0.046 & 1540369 & 1650.056 \\ 32 & 20.609 & \text { VV } & 0.046 & 1632737 & 22.672 & 22.867 \\ 33 & 22.810 & \text { VV } & 0.051 & 1493405 & & \end{array}$


File:

Operator:

Date Acquired

Method File:

Sample Name:

Misc Info:

ALS vial:
C: \CHEMPC \DATA \DJBNO.D

DON. B

21 Jul $94 \quad 3: 03 \mathrm{pm}$

JAFFE.M

ARABIAN CRUDE UNWEATHERED

9 X ORIGINAL

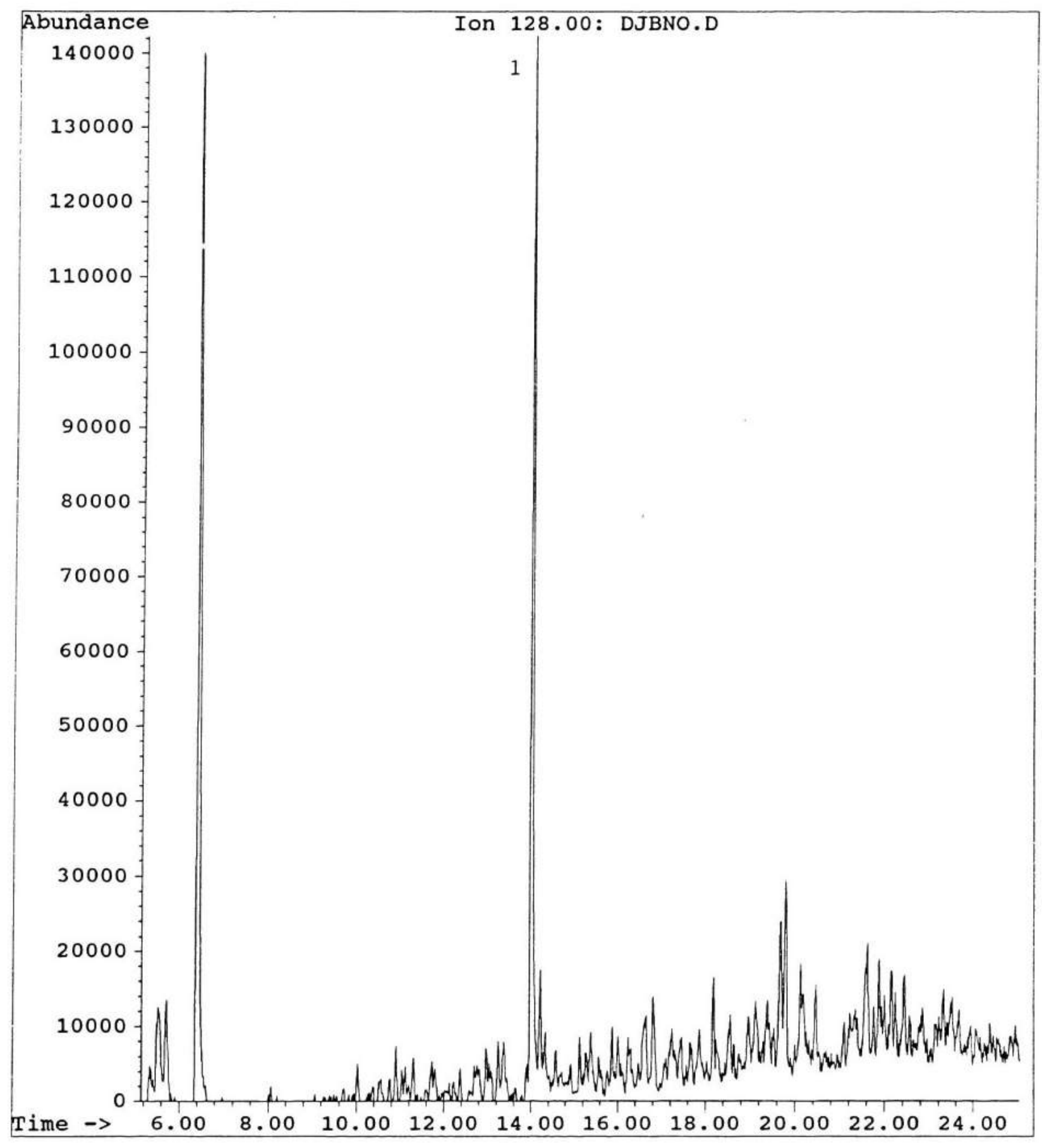


Ion 128.00: DJBNO.D

ARABIAN CRUDE UNWEATHERED

$\begin{array}{crllr}\text { Peak\# } & \text { Ret Time } & \text { Type } & \text { Width } & \text { Area } \\ 1 & 5.712 & \text { VB } & 0.062 & 608035 \\ 2 & 6.415 & \text { BB } & 0.064 & 5661243 \\ 3 & 14.000 & \text { VV } & 0.057 & 5047699 \\ 4 & 14.217 & \text { VV } & 0.047 & 414150 \\ 5 & 18.176 & \text { PV } & 0.044 & 382621 \\ 6 & 19.684 & \text { VV } & 0.070 & 887534 \\ 7 & 19.794 & \text { VV } & 0.062 & 1009582 \\ 8 & 20.134 & \text { PV } & 0.033 & 136503 \\ 9 & 21.616 & \text { PV } & 0.071 & 751310 \\ 10 & 21.880 & \text { PV } & 0.044 & 274630\end{array}$

$\begin{array}{rr}\text { Start Time } & \text { End Time } \\ 5.644 & 5.844 \\ 6.333 & 6.630 \\ 13.939 & 14.154 \\ 14.154 & 14.271 \\ 18.094 & 18.220 \\ 19.580 & 19.738 \\ 19.738 & 19.886 \\ 20.043 & 20.163 \\ 21.485 & 21.686 \\ 21.809 & 21.925\end{array}$


File:

Operator:

Date Acquired

Method File:

Sample Name:

Misc Info:

ALS vial:
C: \CHEMPC \DATA \DJBNO.D

DON. B

21 JUI 94
JAFFE.M

1
$3: 03 \mathrm{pm}$

ARABIAN CRUDE UNWEATHERED

9 X ORIGINAL

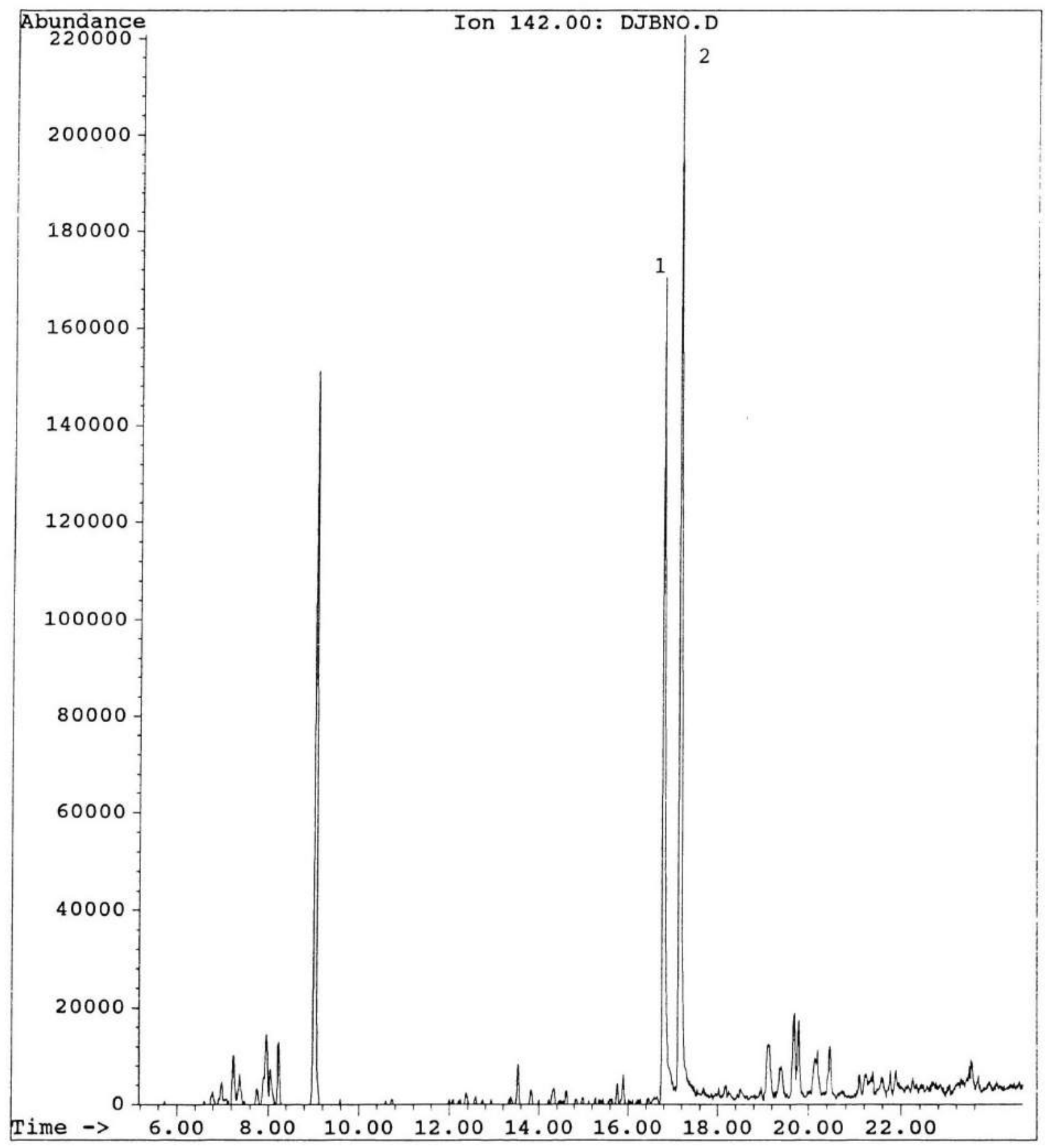


File:

Operator:

Date Acquired:

Method File:

Sample Name:

Misc Info:

ALS vial:
C: \CHEMPC \DATA \DJBNO.D

DON. B

21 Jul 94

JAFFE.M

1
ARABIAN CRUDE UNWEATHERED

9 X ORIGINAL

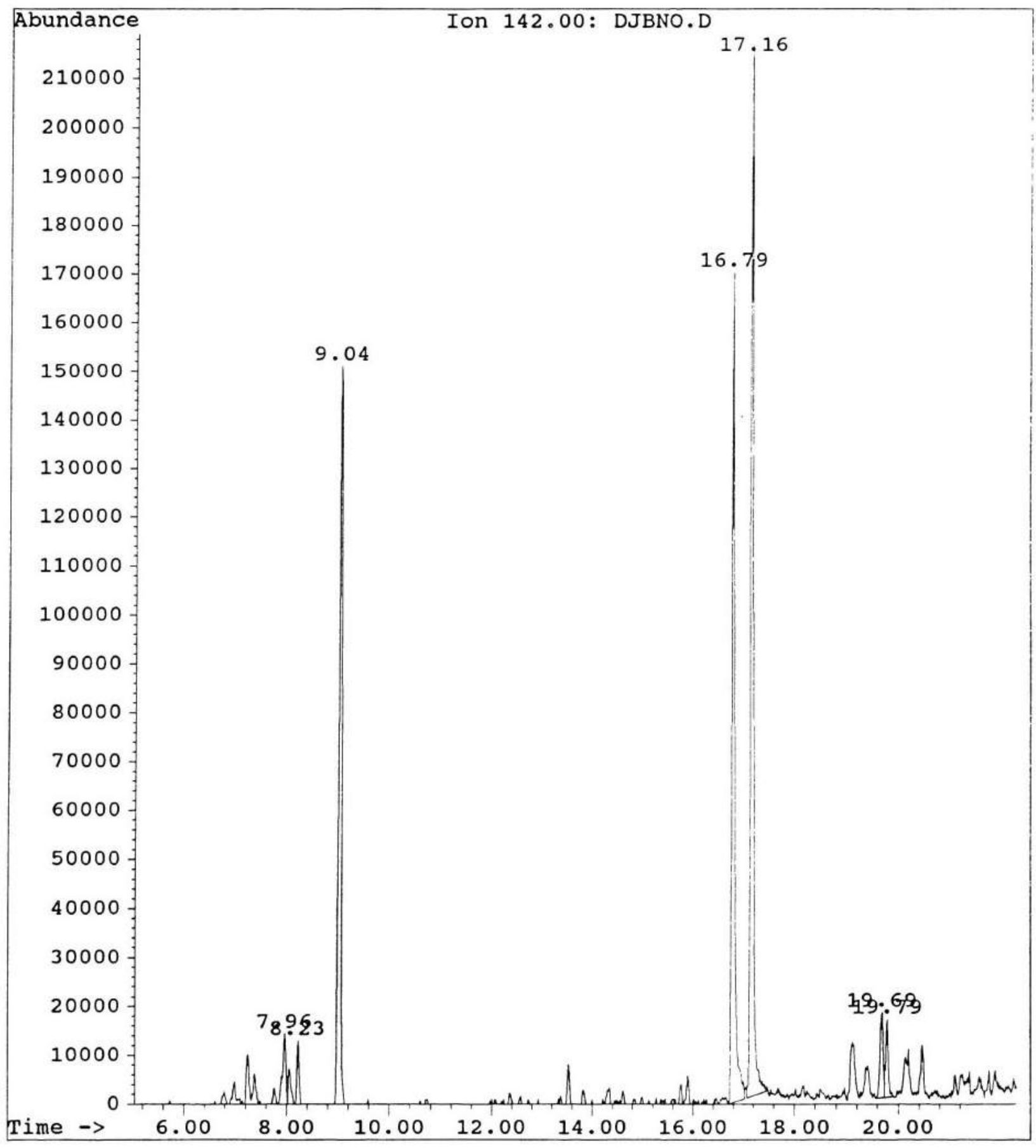


Ion $142.00:$ DJBNO.D

ARABIAN CRUDE UNWEATHERED

$\begin{array}{crlrrrr}\text { Peak\# } & \text { Ret Time } & \text { Type } & \text { Width } & \text { Area } & \text { Start Time } & \text { End Time } \\ 1 & 7.963 & \text { BV } & 0.068 & 666293 & 7.847 & 8.021 \\ 2 & 8.226 & \text { PB } & 0.047 & 364104 & 8.153 & 8.297 \\ 3 & 9.041 & \text { BB } & 0.056 & 5444664 & 8.930 & 9.141 \\ 4 & 16.791 & \text { PV } & 0.058 & 6340792 & 16.707 & 17.022 \\ 5 & 17.162 & \text { VV } & 0.056 & 8156733 & 17.075 & 17.459 \\ 6 & 19.692 & \text { PV } & 0.063 & 709875 & 19.567 & 19.735 \\ 7 & 19.789 & \text { VB } & 0.051 & 538315 & 19.735 & 19.903\end{array}$


File:

operator:

Date Acquired: 21 Jul 94

Method File:

Sample Name:

Misc Info:

ALS vial:
$C: \backslash$ CHEMPC \DATA \DJBNO.D

DON. B

JAFFE.M

1
$3: 03 \mathrm{pm}$

ARABIAN CRUDE UNWEATHERED

9 X ORIGINAL

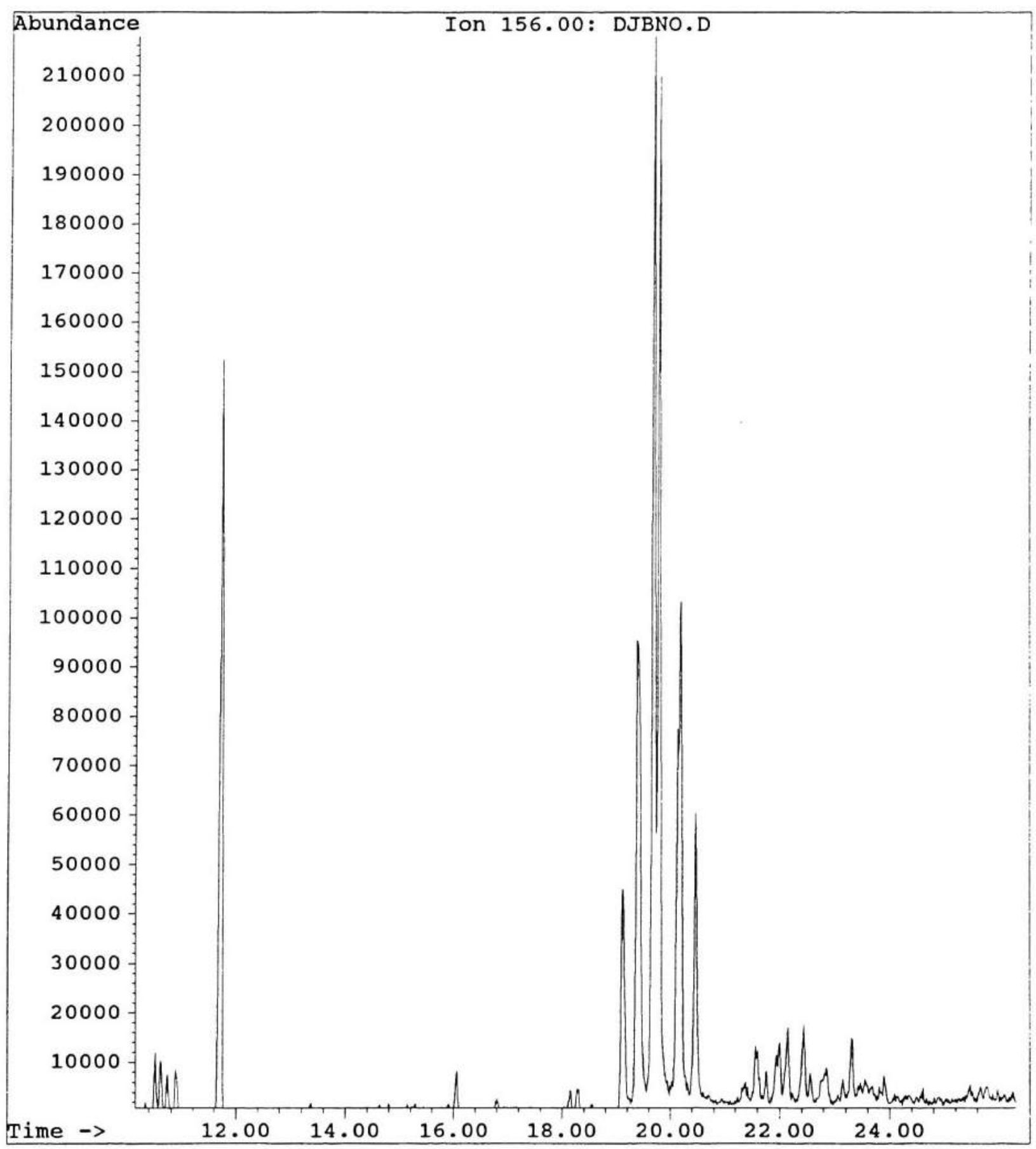


File:

operator:

Date Acquired: 21 Jul 94

Method File:

Sample Name:

Misc Info:

ALS vial:
C: \CHEMPC \DATA \DJBNO.D

DON. B

JAFFE.M

1

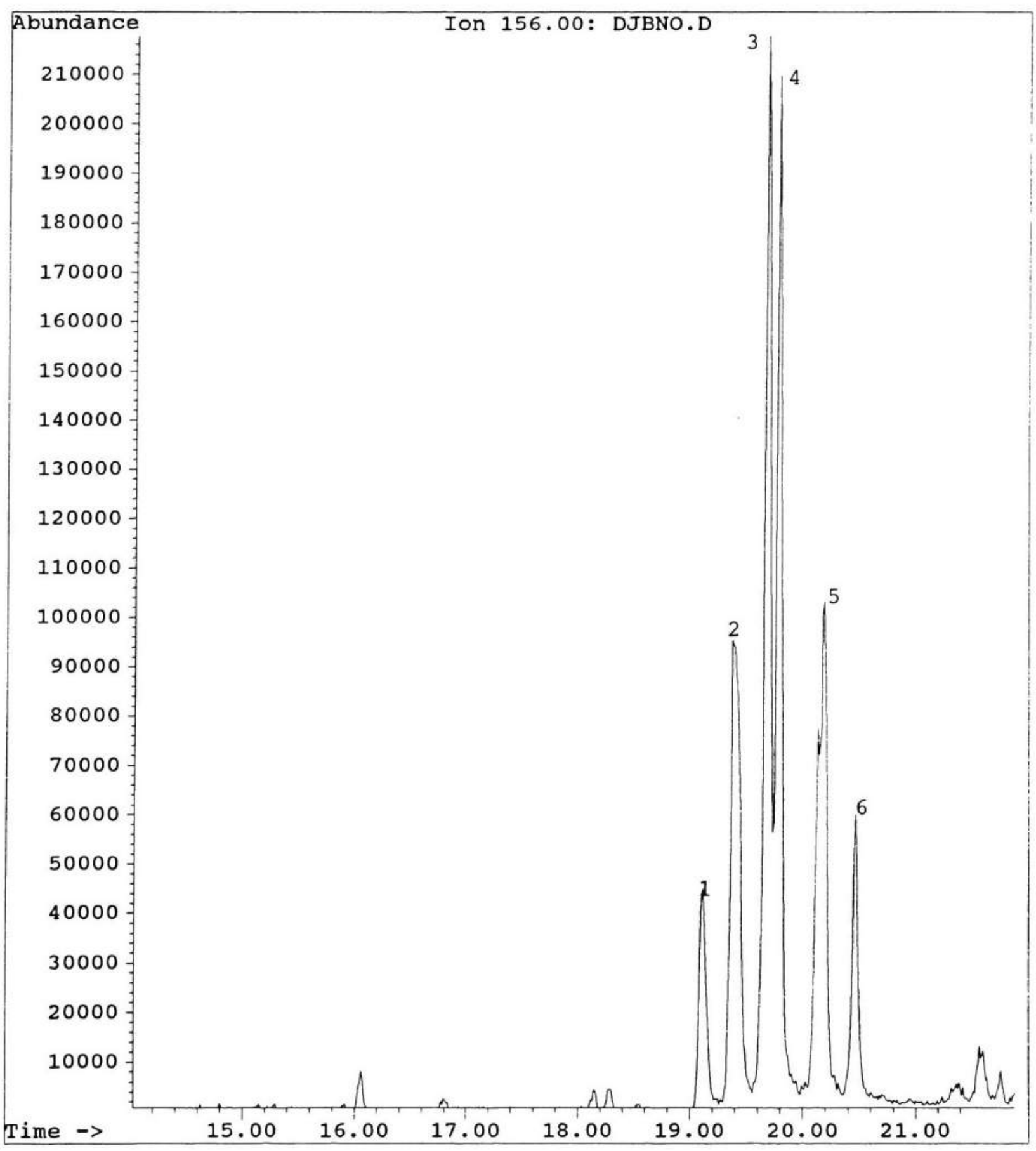


Ion 156.00: DJBNO.D

ARABIAN CRUDE UNWEATHERED

$\begin{array}{crlrrrr}\text { Peak\# } & \text { Ret Time } & \text { Type } & \text { Width } & \text { Area } & \text { Start Time } & \text { End Time } \\ 1 & 11.730 & \text { BB } & 0.054 & 5256141 & 11.623 & 11.795 \\ 2 & 19.114 & \text { BV } & 0.067 & 2047379 & 19.011 & 19.270 \\ 3 & 19.389 & \text { VV } & 0.084 & 5852124 & 19.270 & 19.557 \\ 4 & 19.690 & \text { VV } & 0.061 & 8638323 & 19.557 & 19.740 \\ 5 & 19.790 & \text { VV } & 0.056 & 7481090 & 19.740 & 19.980 \\ 6 & 20.187 & \text { VV } & 0.085 & 6253198 & 19.980 & 20.355 \\ 7 & 20.465 & \text { VB } & 0.058 & 2215504 & 20.355 & 20.669\end{array}$


File:

C: \CHEMPC \DATA \DJBNO.D

Operator: DON. B

Date Acquired: 21 Jul 94

Method File:

JAFFE.M

Sample Name:

Misc Info:

ALS vial:

1

$3: 03 \mathrm{pm}$

ARABIAN CRUDE UNWEATHERED

9 X ORIGINAL

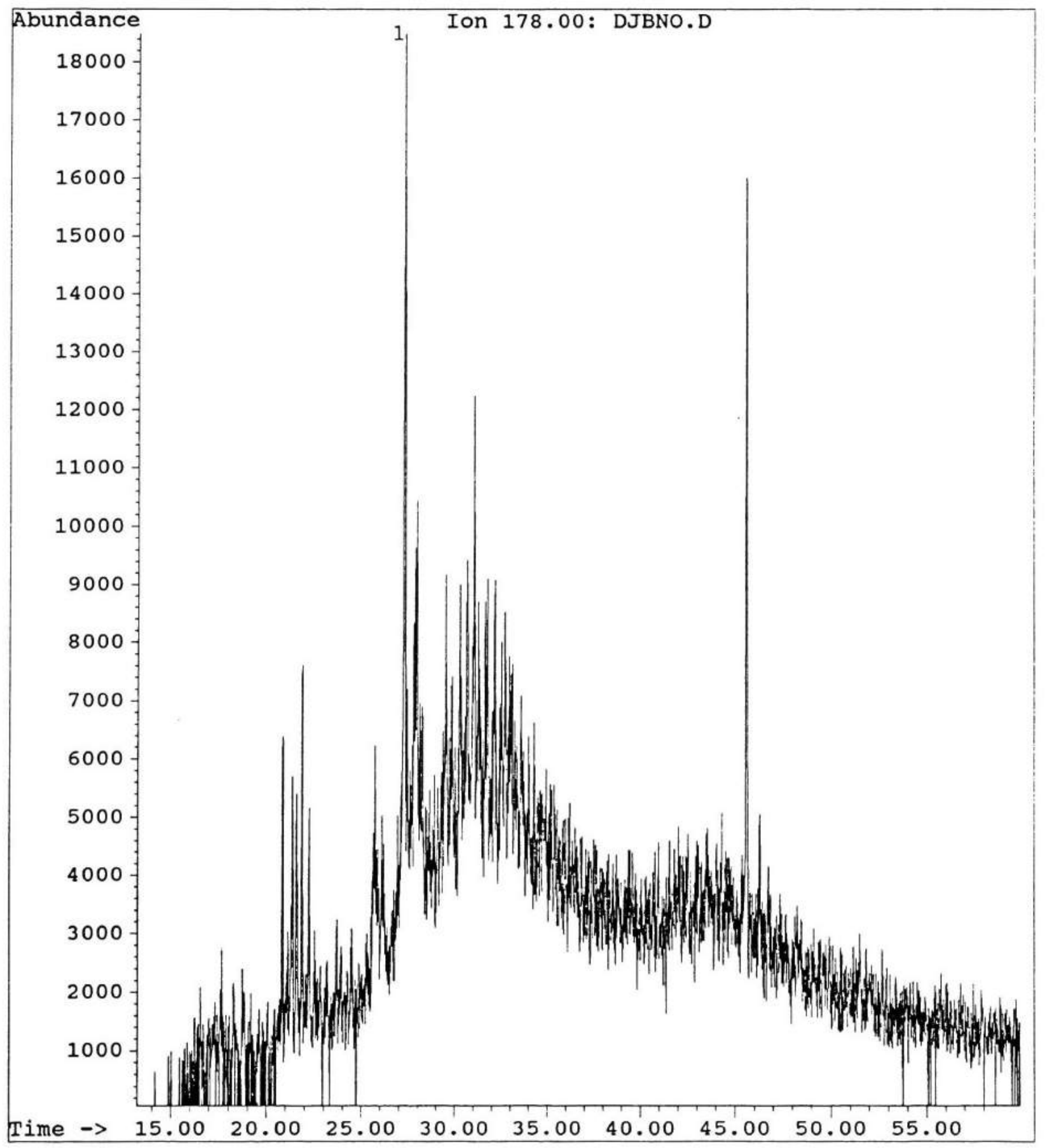


Ion 178.00: DJBNO.D

ARABIAN CRUDE UNWEATHERED

$\begin{array}{crlrr}\text { Peak\# } & \text { Ret Time } & \text { Type } & \text { Width } & \text { Area } \\ 1 & 20.856 & \text { VV } & 0.069 & 280024 \\ 2 & 21.369 & \text { VV } & 0.094 & 338418 \\ 3 & 21.574 & \text { VV } & 0.077 & 271095 \\ 4 & 21.878 & \text { VV } & 0.061 & 263028 \\ 5 & 22.250 & \text { VV } & 0.082 & 243910 \\ 6 & 25.659 & \text { VV } & 0.064 & 86400 \\ 7 & 25.731 & \text { VV } & 0.049 & 118114 \\ 8 & 26.111 & \text { PV } & 0.052 & 70672 \\ 9 & 27.283 & \text { VV } & 0.085 & 855607 \\ 10 & 27.435 & \text { VV } & 0.054 & 112801 \\ 11 & 27.792 & \text { PV } & 0.054 & 148555 \\ 12 & 27.877 & \text { VV } & 0.052 & 166373 \\ 13 & 27.971 & \text { VV } & 0.052 & 165297 \\ 14 & 29.375 & \text { VV } & 0.032 & 49440 \\ 15 & 29.522 & \text { VV } & 0.070 & 259556 \\ 16 & 29.819 & \text { VV } & 0.049 & 89714 \\ 17 & 29.873 & \text { VV } & 0.052 & 120720 \\ 18 & 30.300 & \text { VV } & 0.060 & 220654 \\ 19 & 30.674 & \text { VV } & 0.092 & 320976 \\ 20 & 30.966 & \text { VV } & 0.066 & 156408 \\ 21 & 31.057 & \text { VV } & 0.069 & 402684 \\ 22 & 31.280 & \text { VV } & 0.055 & 176713 \\ 23 & 31.654 & \text { VV } & 0.066 & 168417 \\ 24 & 31.772 & \text { VV } & 0.074 & 224181 \\ 25 & 32.162 & \text { VV } & 0.070 & 226171 \\ 26 & 32.421 & \text { PV } & 0.051 & 62768 \\ 27 & 32.533 & \text { VV } & 0.057 & 109468 \\ 28 & 32.715 & \text { VV } & 0.070 & 189056 \\ 29 & 32.953 & \text { VV } & 0.043 & 45720 \\ 30 & 33.588 & \text { BV } & 0.055 & 83956 \\ 31 & 45.611 & \text { VV } & 0.074 & 683938 \\ 32 & 46.297 & \text { VV } & 0.055 & 90077\end{array}$

$\begin{array}{rr}\text { Start Time } & \text { End Time } \\ 20.749 & 20.927 \\ 21.190 & 21.445 \\ 21.498 & 21.686 \\ 21.798 & 21.945 \\ 22.126 & 22.368 \\ 25.619 & 25.697 \\ 25.697 & 25.785 \\ 26.013 & 26.170 \\ 27.054 & 27.386 \\ 27.386 & 27.513 \\ 27.741 & 27.838 \\ 27.838 & 27.924 \\ 27.924 & 28.040 \\ 29.351 & 29.416 \\ 29.455 & 29.674 \\ 29.773 & 29.844 \\ 29.844 & 29.969 \\ 30.238 & 30.360 \\ 30.614 & 30.754 \\ 30.863 & 30.982 \\ 30.982 & 31.138 \\ 31.229 & 31.332 \\ 31.548 & 31.703 \\ 31.703 & 31.866 \\ 32.073 & 32.218 \\ 32.353 & 32.463 \\ 32.463 & 32.556 \\ 32.643 & 32.836 \\ 32.930 & 33.018 \\ 33.540 & 33.634 \\ 45.491 & 45.721 \\ 46.175 & 46.354\end{array}$


File:

operator:

Date Acquired: 21 Jul 94

Method File:

Sample Name:

Misc Info:

ALS vial:
$C: \backslash C H E M P C \backslash D A T A \backslash D J B N O . D$

DON. B

JAFFE.M

1
$3: 03 \mathrm{pm}$

ARABIAN CRUDE UNWEATHERED

9 X ORIGINAL

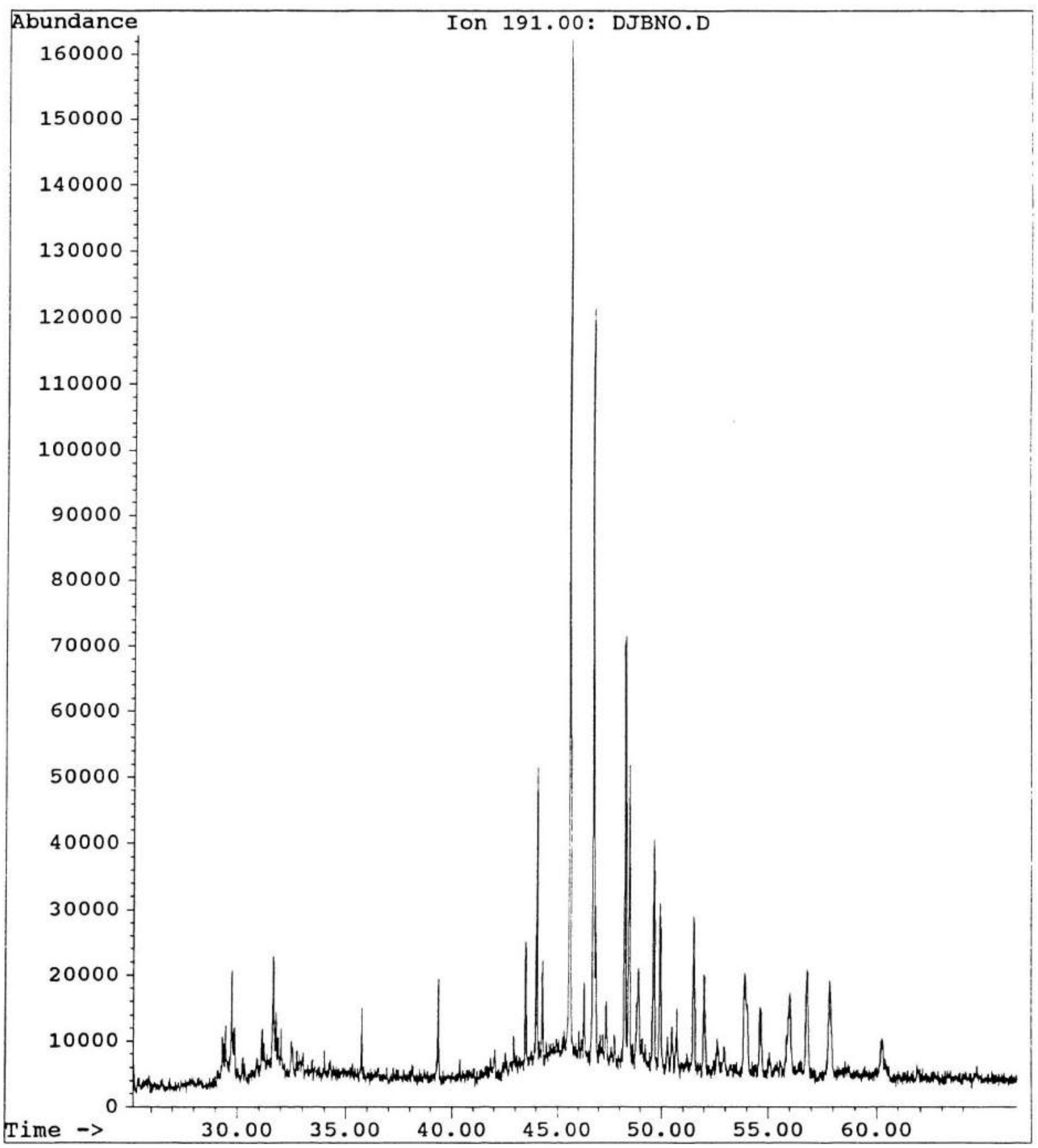


File:

operator:

Date Acquired:

Method File:

Sample Name:

Misc Info:

ALS vial:

C: \CHEMPC \DATA \DJBNO.D

DON. B
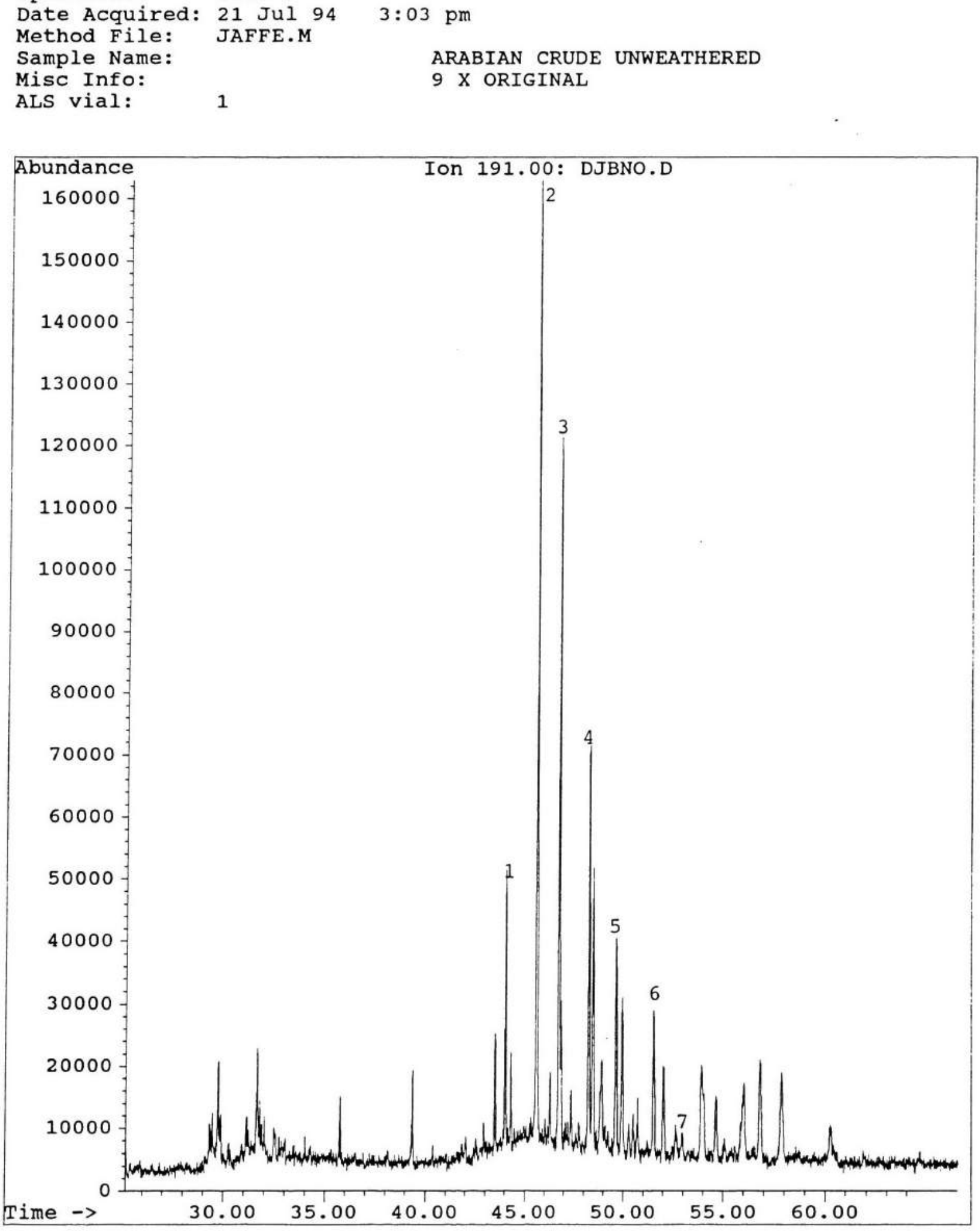
Ion 191.00: DJBNO.D

ARABIAN CRUDE UNWEATHERED

$\begin{array}{crlrrrr}\text { Peak\# } & \text { Ret Time } & \text { Type } & \text { Width } & \text { Area } & \text { Start Time } & \text { End Time } \\ 1 & 29.746 & \text { PV } & 0.052 & 439574 & 29.618 & 29.805 \\ 2 & 31.650 & \text { PV } & 0.056 & 560410 & 31.565 & 31.735 \\ 3 & 35.759 & \text { VV } & 0.044 & 279828 & 35.702 & 35.835 \\ 4 & 39.356 & \text { VV } & 0.053 & 548868 & 39.289 & 39.481 \\ 5 & 43.507 & \text { BV } & 0.056 & 678613 & 43.401 & 43.632 \\ 6 & 44.032 & \text { VV } & 0.051 & 1457513 & 43.963 & 44.107 \\ 7 & 44.307 & \text { VV } & 0.066 & 557561 & 44.216 & 44.369 \\ 8 & 45.610 & \text { VV } & 0.063 & 6422436 & 45.524 & 45.864 \\ 9 & 46.302 & \text { VV } & 0.061 & 431158 & 46.229 & 46.405 \\ 10 & 46.750 & \text { PV } & 0.063 & 4688143 & 46.601 & 46.818 \\ 11 & 46.845 & \text { VV } & 0.061 & 849752 & 46.818 & 46.955 \\ 12 & 47.366 & \text { VV } & 0.067 & 458954 & 47.257 & 47.502 \\ 13 & 48.266 & \text { VV } & 0.067 & 2977944 & 48.153 & 48.369 \\ 14 & 48.464 & \text { VV } & 0.080 & 2380720 & 48.369 & 48.614 \\ 15 & 48.911 & \text { VV } & 0.109 & 1185106 & 48.685 & 48.992 \\ 16 & 49.633 & \text { PV } & 0.082 & 1908582 & 49.478 & 49.735 \\ 17 & 49.935 & \text { VV } & 0.083 & 1509954 & 49.832 & 50.176 \\ 18 & 50.731 & \text { VV } & 0.078 & 521083 & 50.600 & 50.894 \\ 19 & 51.519 & \text { VV } & 0.084 & 1482401 & 51.357 & 51.701 \\ 20 & 52.014 & \text { PV } & 0.086 & 928180 & 51.902 & 52.152 \\ 21 & 53.919 & \text { VV } & 0.139 & 1581456 & 53.807 & 54.144 \\ 22 & 54.643 & \text { BV } & 0.094 & 683980 & 54.479 & 54.797 \\ 23 & 56.028 & \text { VV } & 0.118 & 1197903 & 55.895 & 56.212 \\ 24 & 56.815 & \text { VV } & 0.105 & 1274021 & 56.620 & 56.954 \\ 25 & 57.864 & \text { VV } & 0.115 & 1316052 & 57.735 & 58.074\end{array}$


File:

operator:

Date Acquired:

Method File:

Sample Name:

Misc Info:

ALS vial:
C: $\backslash$ CHEMPC $\backslash D A T A \backslash D J B N O . D$

DON. B

21 Jul 94

JAFFE.M

1

\section{$3: 03 \mathrm{pm}$}

ARABIAN CRUDE UNWEATHERED

9 X ORIGINAL

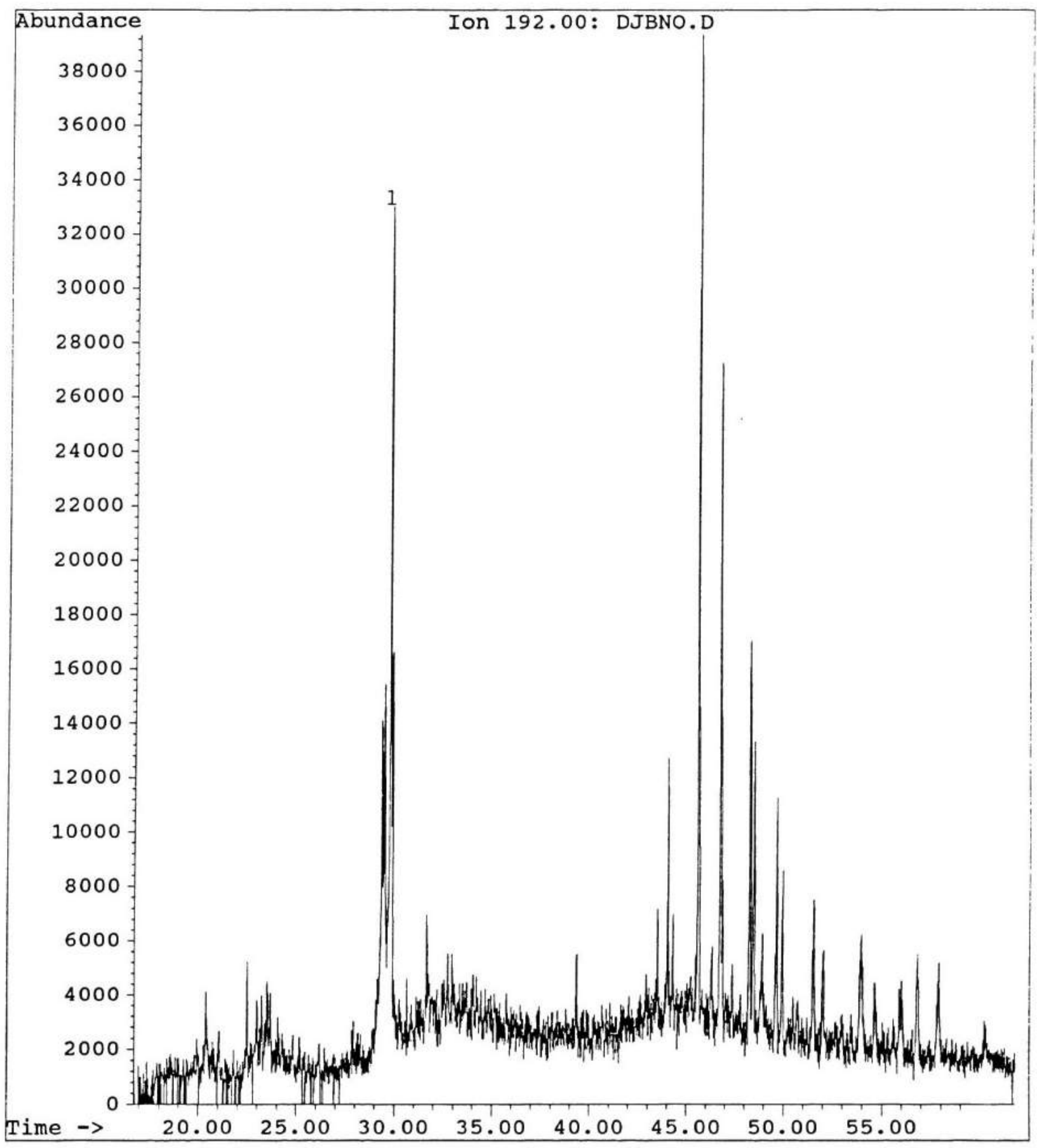


File:

operator:

Date Acquired: 21 Jul 94

Method File:

Sample Name:

Misc Info:

ALS vial:
C: $\backslash$ CHEMPC $\backslash$ DATA $\backslash$ DJBNO.D

DON. B

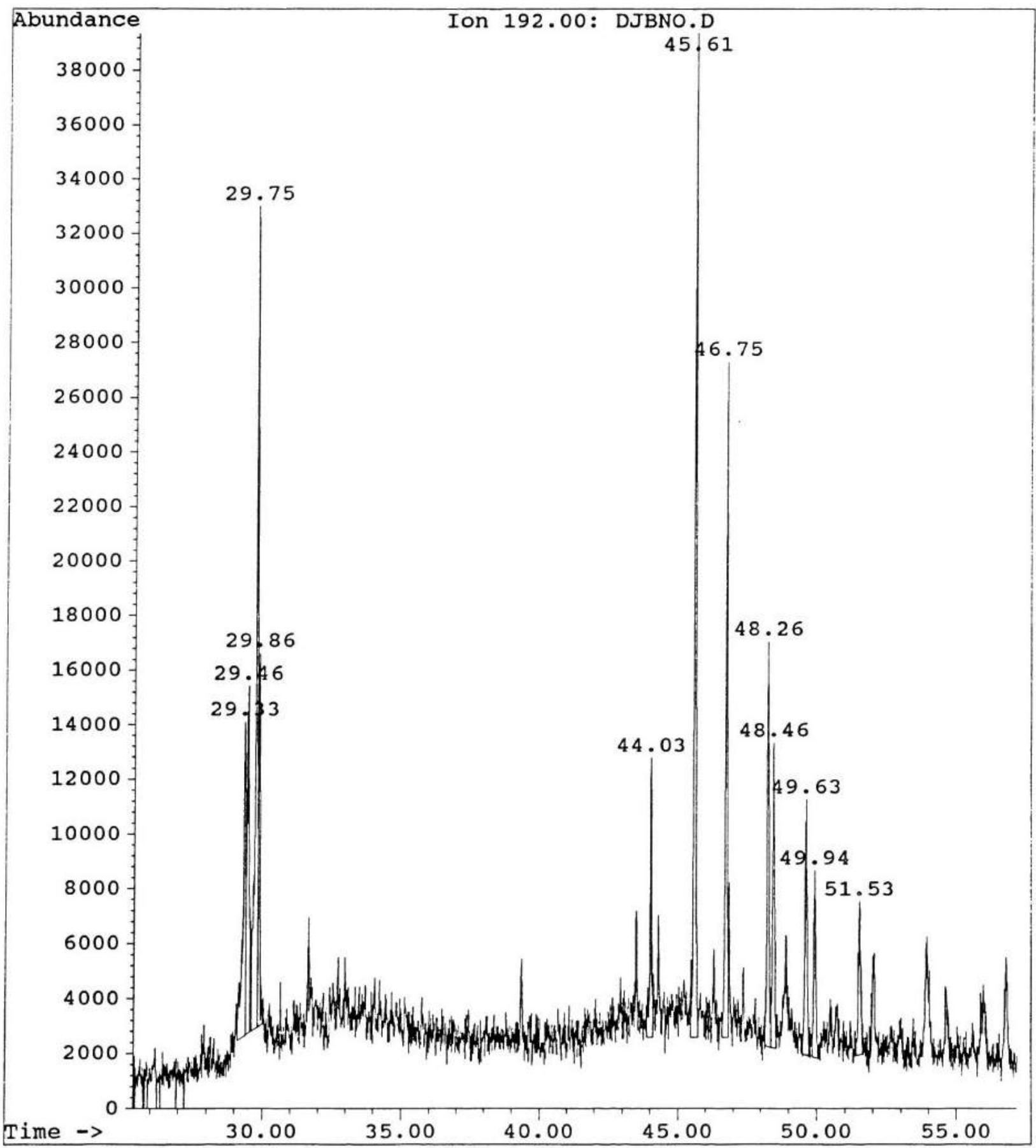


Ion 192.00: DJBNO.D

ARABIAN CRUDE UNWEATHERED

$\begin{array}{crlrrrr}\text { Peak\# } & \text { Ret Time } & \text { Type } & \text { width } & \text { Area } & \text { Start Time } & \text { End Time } \\ 1 & 29.328 & \text { VV } & 0.092 & 758529 & 29.103 & 29.394 \\ 2 & 29.456 & \text { VV } & 0.083 & 721210 & 29.394 & 29.554 \\ 3 & 29.745 & \text { VV } & 0.070 & 1469423 & 29.602 & 29.812 \\ 4 & 29.856 & \text { VV } & 0.068 & 629511 & 29.812 & 30.006 \\ 5 & 44.031 & \text { VV } & 0.061 & 405305 & 43.877 & 44.095 \\ 6 & 45.609 & \text { VV } & 0.066 & 1624277 & 45.471 & 45.724 \\ 7 & 46.751 & \text { VV } & 0.060 & 1044278 & 46.601 & 46.816 \\ 8 & 48.261 & \text { VV } & 0.068 & 713019 & 48.160 & 48.373 \\ 9 & 48.459 & \text { VV } & 0.068 & 550510 & 48.373 & 48.562 \\ 10 & 49.627 & \text { VV } & 0.075 & 473619 & 49.470 & 49.736 \\ 11 & 49.944 & \text { VV } & 0.079 & 329270 & 49.849 & 50.066 \\ 12 & 51.529 & \text { PV } & 0.076 & 320983 & 51.394 & 51.663\end{array}$


File:

operator:

Date Acquired:

Method File:

Sample Name:

Misc Info:

ALS vial:
C: \CHEMPC \DATA \DJBNO.D

DON. B

21 Jul 94

JAFFE.M

1
3:03 pm

ARABIAN CRUDE UNWEATHERED

9 X ORIGINAL

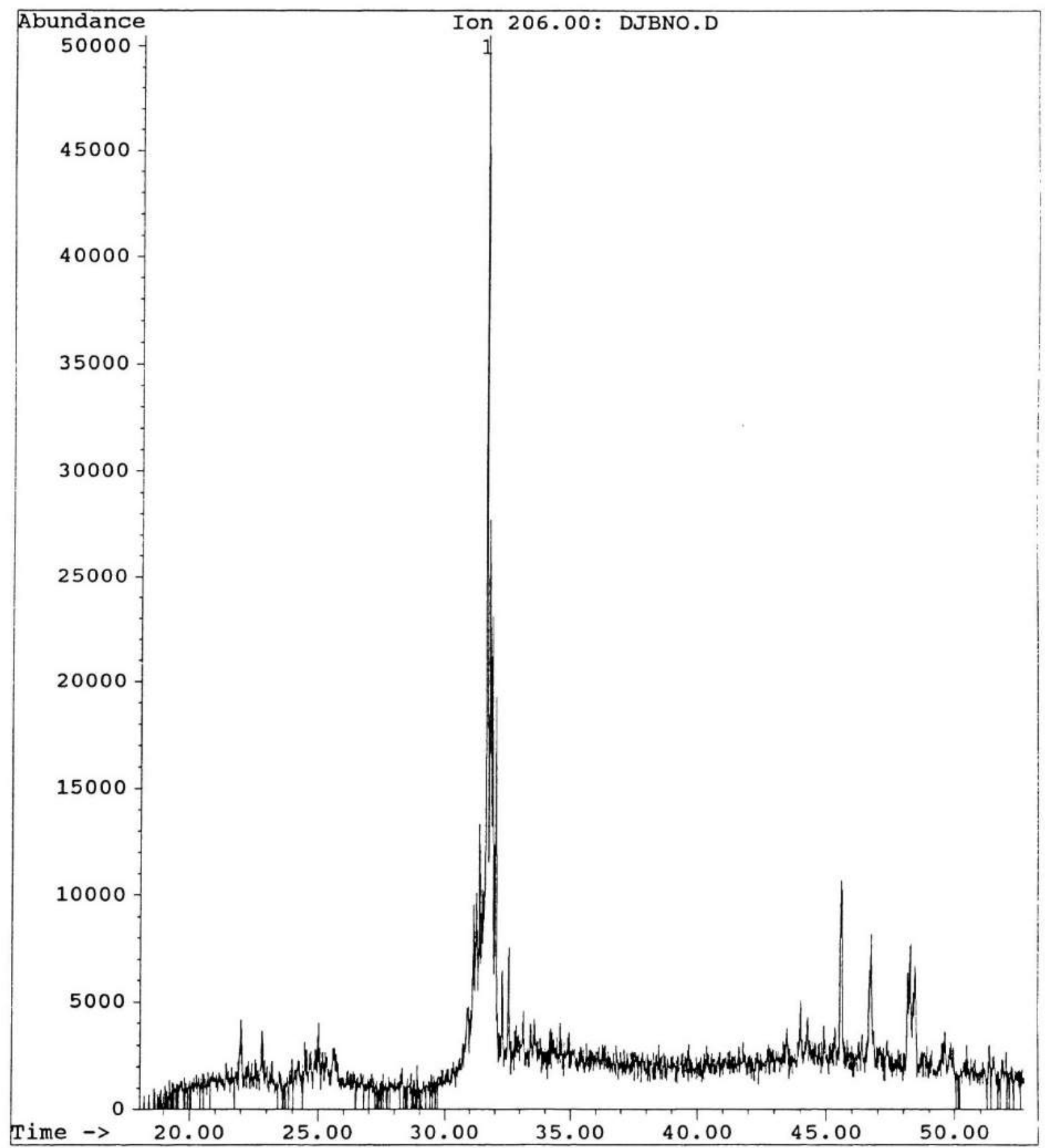


File:

Operator:

Date Acquired:

Method File:

Sample Name:

Misc Info:

ALS vial:
C: \CHEMPC \DATA \DJBNO.D

DON. B

21 Jul 94 3:03 pm

JAFFE.M

1
ARABIAN CRUDE UNWEATHERED

9 X ORIGINAL

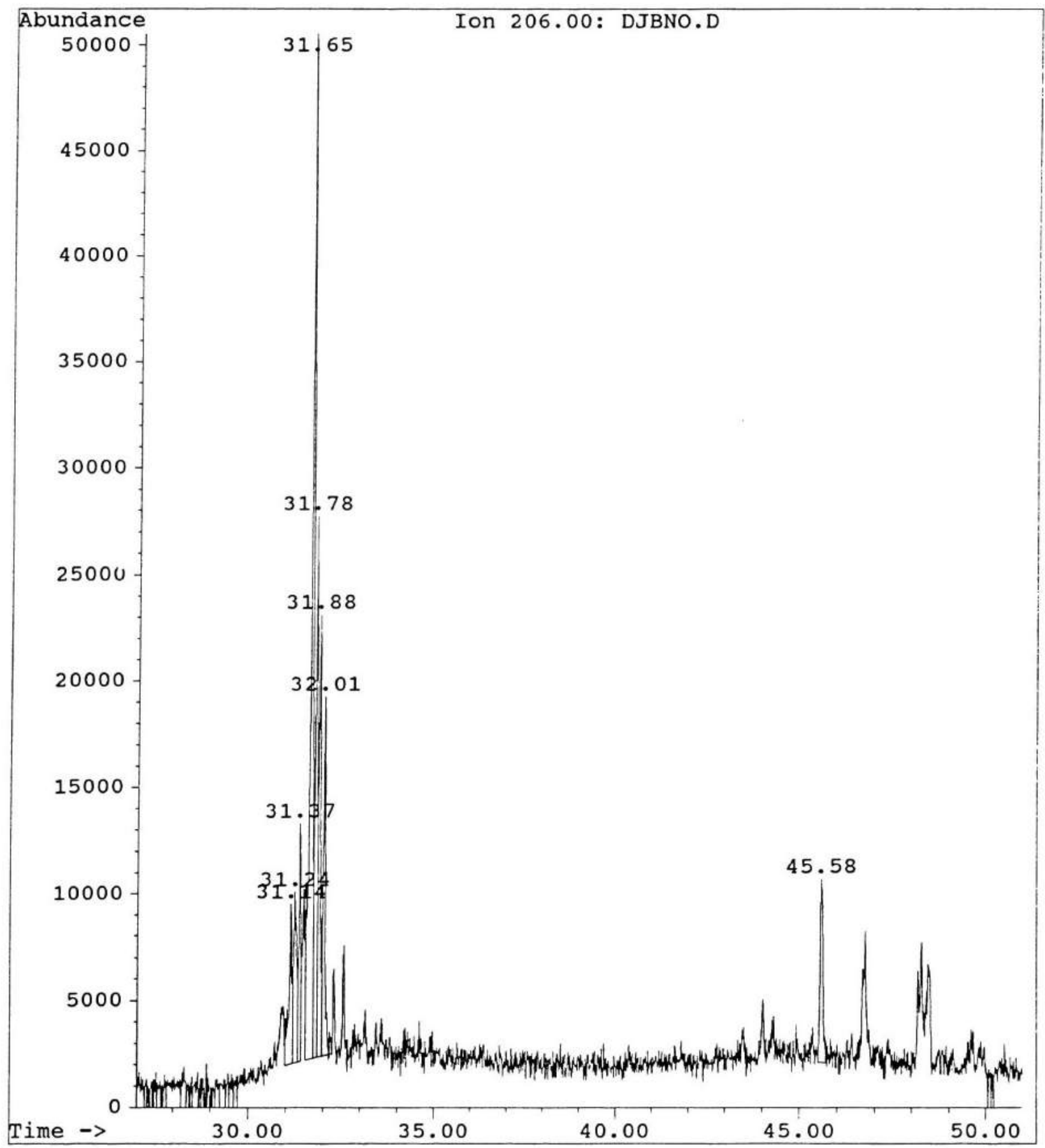


ARABIAN CRUDE UNWEATHERED

$\begin{array}{crlrrrr}\text { Peak\# } & \text { Ret Time } & \text { Type } & \text { Width } & \text { Area } & \text { Start Time } & \text { End Time } \\ 1 & 31.136 & \text { VV } & 0.077 & 399847 & 30.982 & 31.189 \\ 2 & 31.239 & \text { VV } & 0.075 & 439166 & 31.189 & 31.318 \\ 3 & 31.369 & \text { VV } & 0.053 & 406179 & 31.318 & 31.414 \\ 4 & 31.651 & \text { VV } & 0.068 & 2327208 & 31.524 & 31.724 \\ 5 & 31.776 & \text { VV } & 0.060 & 1065349 & 31.724 & 31.836 \\ 6 & 31.879 & \text { VV } & 0.059 & 862282 & 31.836 & 31.964 \\ 7 & 32.009 & \text { VV } & 0.057 & 663452 & 31.964 & 32.236 \\ 8 & 45.583 & \text { VV } & 0.091 & 472303 & 45.497 & 45.739\end{array}$


File:

Operator:

Date Acquired: 21 Jul 94

Method File: JAFFE.M

Sample Name:

Misc Info:

ALS vial:

BOYE
C: \CHEMPC \DATA \DJB8H.D

$9: 25 \mathrm{pm}$

ARABIAN CRUDE 8 HRS WEATHERING

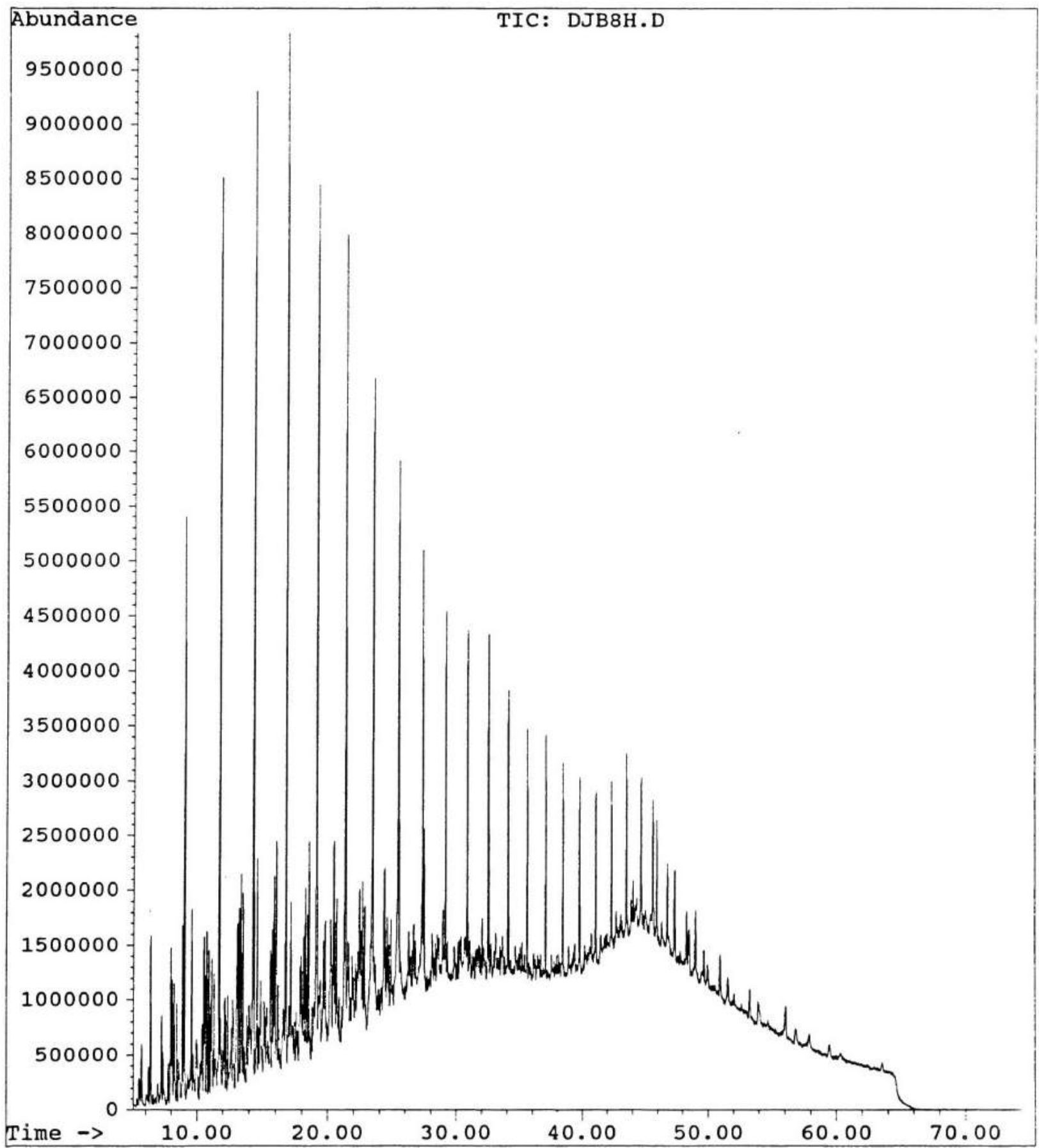


File:

Operator:

Date Acquired: 21 Jul 94

Method File:

Sample Name:

Misc Info:

ALS vial:

BOYE

JAFFE.M

1
C: \CHEMPC \DATA \DJB8H.D

9:25 pm

ARABIAN CRUDE 8 HRS WEATHERING

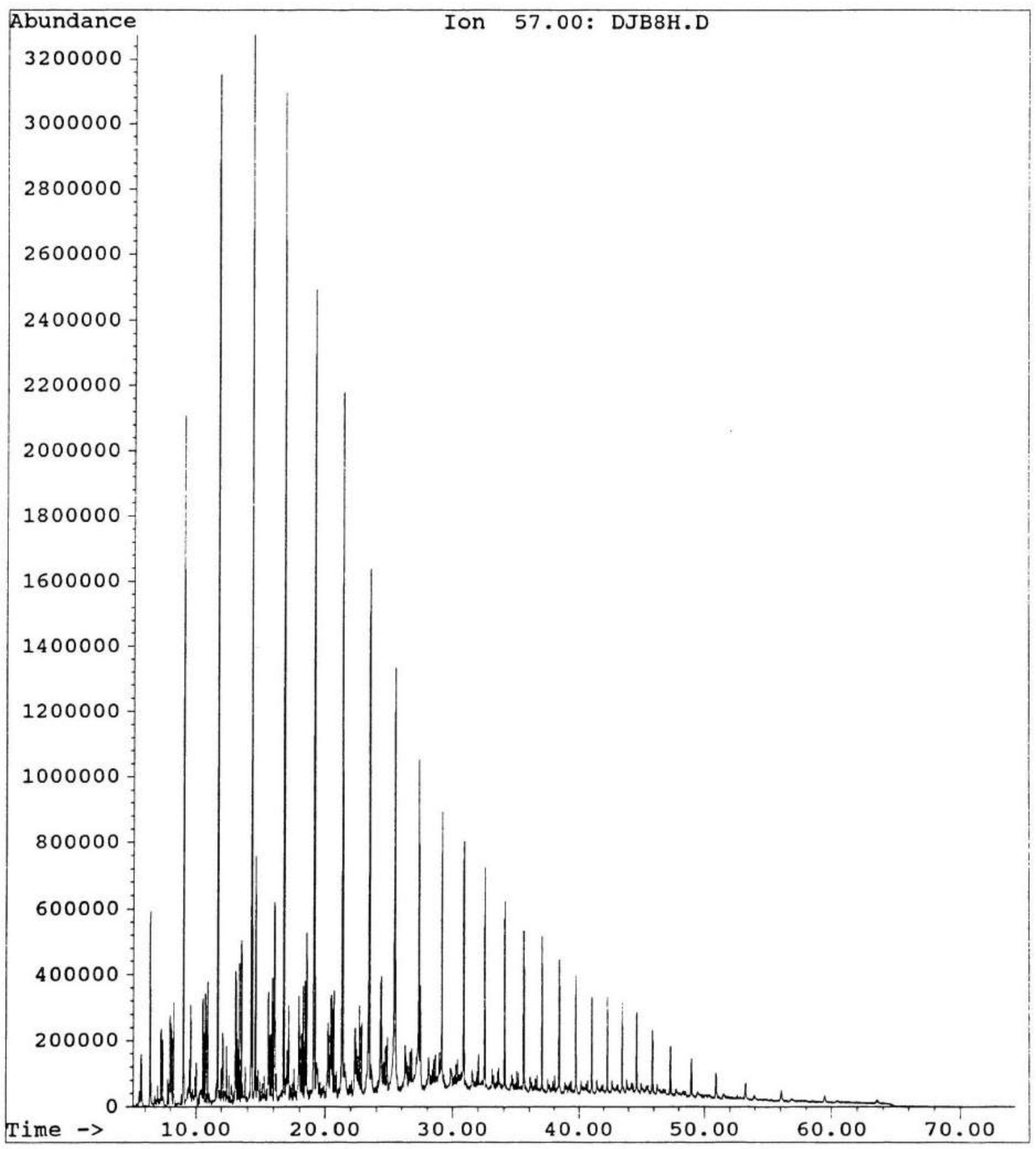


ARABIAN CRUDE 8 HRS WEATHERING

\begin{tabular}{|c|c|c|c|c|}
\hline Peak\# & Ret Time & Type & width & Area \\
\hline 1 & 5.693 & VB & 0.069 & 7035124 \\
\hline 2 & 6.387 & BV & 0.057 & 20995865 \\
\hline 3 & 7.233 & VV & 0.054 & 8277905 \\
\hline 4 & 7.371 & VB & 0.052 & 7028294 \\
\hline 5 & 7.759 & BV & 0.056 & 3096337 \\
\hline 6 & 7.951 & vV & 0.055 & 10057375 \\
\hline 7 & 8.046 & vV & 0.053 & 8650131 \\
\hline 8 & 8.213 & VV & 0.053 & 10656499 \\
\hline 9 & 9.011 & PV & 0.046 & 60991767 \\
\hline 10 & 9.478 & vV & 0.054 & 4849581 \\
\hline 11 & 9.577 & VV & 0.044 & 8560773 \\
\hline 12 & 10.017 & VB & 0.049 & 4293152 \\
\hline 13 & 10.504 & VV & 0.045 & 9145943 \\
\hline 14 & 10.602 & vV & 0.044 & 5837024 \\
\hline 15 & 10.725 & VV & 0.048 & 9959635 \\
\hline 16 & 10.889 & VV & 0.049 & 11711909 \\
\hline 17 & 11.709 & vV & 0.047 & 91012764 \\
\hline 18 & 11.980 & VV & 0.048 & 3065433 \\
\hline 19 & 12.072 & VV & 0.057 & 8166252 \\
\hline 20 & 12.365 & PV & 0.046 & 4800774 \\
\hline 21 & 13.081 & vV & 0.058 & 15381123 \\
\hline 22 & 13.226 & vV & 0.043 & 5033875 \\
\hline 23 & 13.366 & vV & 0.042 & 10899934 \\
\hline 24 & 13.525 & PV & 0.041 & 12134940 \\
\hline 25 & 13.824 & vV & 0.058 & 4096614 \\
\hline 26 & 14.323 & VV & 0.050 & 98322412 \\
\hline 27 & 14.525 & VV & 0.046 & 3008573 \\
\hline 28 & 14.612 & VV & 0.041 & 18635409 \\
\hline 29 & 15.580 & vV & 0.062 & 14179765 \\
\hline 30 & 15.754 & VV & 0.048 & 6318057 \\
\hline 31 & 15.894 & vV & 0.046 & 10978054 \\
\hline 32 & 16.062 & PV & 0.065 & 23048394 \\
\hline 33 & 16.808 & vV & 0.047 & 97163952 \\
\hline 34 & 17.027 & VV & 0.051 & 5116747 \\
\hline 35 & 17.164 & vV & 0.051 & 9804026 \\
\hline 36 & 17.573 & PV & 0.047 & 2902056 \\
\hline 37 & 17.951 & PV & 0.055 & 11422453 \\
\hline 38 & 18.022 & VV & 0.046 & 4643487 \\
\hline 39 & 18.146 & vV & 0.046 & 5822821 \\
\hline 40 & 18.288 & VV & 0.047 & 10329864 \\
\hline 41 & 18.441 & PV & 0.048 & 10308599 \\
\hline 42 & 18.551 & vV & 0.043 & 13073351 \\
\hline 43 & 19.160 & BV & 0.055 & 88903425 \\
\hline 44 & 19.315 & VV & 0.089 & 6487545 \\
\hline 45 & 20.214 & PV & 0.065 & 10818821 \\
\hline 46 & 20.282 & vV & 0.045 & 3639874 \\
\hline 47 & 20.468 & vV & 0.066 & 14285848 \\
\hline 48 & 20.553 & vV & 0.044 & 7133560 \\
\hline 49 & 20.701 & vV & 0.044 & 8232121 \\
\hline 50 & 21.377 & PV & 0.052 & 74795875 \\
\hline 51 & 21.499 & vV & 0.050 & 1689507 \\
\hline 52 & 22.354 & PV & 0.075 & 11327238 \\
\hline 53 & 22.438 & vV & 0.070 & 5854250 \\
\hline 54 & 22.563 & vV & 0.049 & 3960388 \\
\hline 55 & 22.695 & vV & 0.049 & 8420291 \\
\hline 56 & 22.842 & vV & 0.048 & 6889274 \\
\hline
\end{tabular}

Start Time

5.605

6.323

7.173

7.317

7.646

7.842

8.003

8.158

8.910

9.428

9.530

9.972

10.450

10.556

10.660

10.827

11.615

11.919

12.024

12.314

12.981

13.170

13.291

13.458

13.687

14.190

14.406

14.553

15.483

15.678

15.816

15.969

16.669

16.985

17.075

17.462

17.773

17.991

18.082

18.206

18.355

18.489

18.992

19.243

20.071

20.256

20.329

20.510

20.617

21.141

21.446

22.147

22.397

22.516

22.617

22.752
End Time

5.892

6.553

7.317

7.559

7.842

8.003

8.158

8.373

9.126

9.530

9.648

10.147

10.556

10.660

10.827

10.997

11.766

12.024

12.168

12.453

13.170

13.291

13.458

13.576

13.899

14.406

14.553

14.665

15.678

15.816

15.969

16.175

16.887

17.075

17. 232

17.616

17.991

18.082

18.206

18.355

18.489

18.610

19.243

19.403

20.256

20.329

20.510

20.617

20.750

21.446

21.553

22.397

22.516

22.617

22. 752

22.914 


$\begin{array}{lrlrr}57 & 23.476 & \text { VV } & 0.059 & 64601672 \\ 58 & 24.387 & \text { VV } & 0.081 & 19869995 \\ 59 & 24.726 & \text { VV } & 0.053 & 4767775 \\ 60 & 24.870 & \text { VV } & 0.047 & 5045427 \\ 61 & 25.466 & \text { VV } & 0.070 & 64929327 \\ 62 & 26.257 & \text { PV } & 0.060 & 5084242 \\ 63 & 26.654 & \text { PV } & 0.044 & 2753712 \\ 64 & 26.794 & \text { VV } & 0.039 & 2492988 \\ 65 & 27.357 & \text { VV } & 0.055 & 38872630 \\ 66 & 27.454 & \text { VV } & 0.063 & 12274406 \\ 67 & 28.084 & \text { VV } & 0.067 & 4167740 \\ 68 & 28.628 & \text { VV } & 0.046 & 2616589 \\ 69 & 28.933 & \text { VV } & 0.061 & 3756549 \\ 70 & 29.156 & \text { VV } & 0.045 & 24957228 \\ 71 & 30.872 & \text { BV } & 0.043 & 19297805 \\ 72 & 32.038 & \text { VV } & 0.050 & 3210421 \\ 73 & 32.513 & \text { VV } & 0.043 & 17653543 \\ 74 & 34.083 & \text { PV } & 0.046 & 15984975 \\ 75 & 35.587 & \text { PV } & 0.041 & 12794190 \\ 76 & 37.031 & \text { PV } & 0.043 & 12181306 \\ 77 & 38.419 & \text { VV } & 0.049 & 12385312 \\ 78 & 39.755 & \text { BV } & 0.046 & 11089453 \\ 79 & 41.042 & \text { PV } & 0.046 & 8921454 \\ 80 & 42.286 & \text { PV } & 0.045 & 8582920 \\ 81 & 43.487 & \text { PV } & 0.046 & 8102568 \\ 82 & 44.652 & \text { PV } & 0.047 & 7311396 \\ 83 & 45.903 & \text { PV } & 0.051 & 6368945 \\ 84 & 47.328 & \text { BV } & 0.064 & 6190212 \\ 85 & 48.980 & \text { BB } & 0.074 & 6018890\end{array}$

$\begin{array}{ll}23.276 & 23.532 \\ 24.171 & 24.441 \\ 24.639 & 24.780 \\ 24.780 & 24.917 \\ 25.070 & 25.604 \\ 26.139 & 26.304 \\ 26.571 & 26.735 \\ 26.735 & 26.844 \\ 27.172 & 27.402 \\ 27.402 & 27.618 \\ 27.999 & 28.144 \\ 28.548 & 28.682 \\ 28.826 & 28.987 \\ 28.987 & 29.211 \\ 30.770 & 30.983 \\ 31.969 & 32.103 \\ 32.397 & 32.568 \\ 33.893 & 34.138 \\ 35.439 & 35.644 \\ 36.913 & 37.084 \\ 38.225 & 38.581 \\ 39.635 & 39.904 \\ 40.927 & 41.187 \\ 42.101 & 42.393 \\ 43.301 & 43.564 \\ 44.398 & 44.732 \\ 45.684 & 45.981 \\ 47.167 & 47.608 \\ 48.672 & 49.247\end{array}$


File:

operator:

Date Acquired: 1 Jul

Method File: JAFFE.M

Sample Name:

Misc Info:

ALS vial:

BOYE

1
$C: \backslash C H E M P C \backslash D A T A \backslash D J B 8 H . D$

$9: 25 \mathrm{pm}$

ARABIAN CRUDE 8 HRS WEATHERING

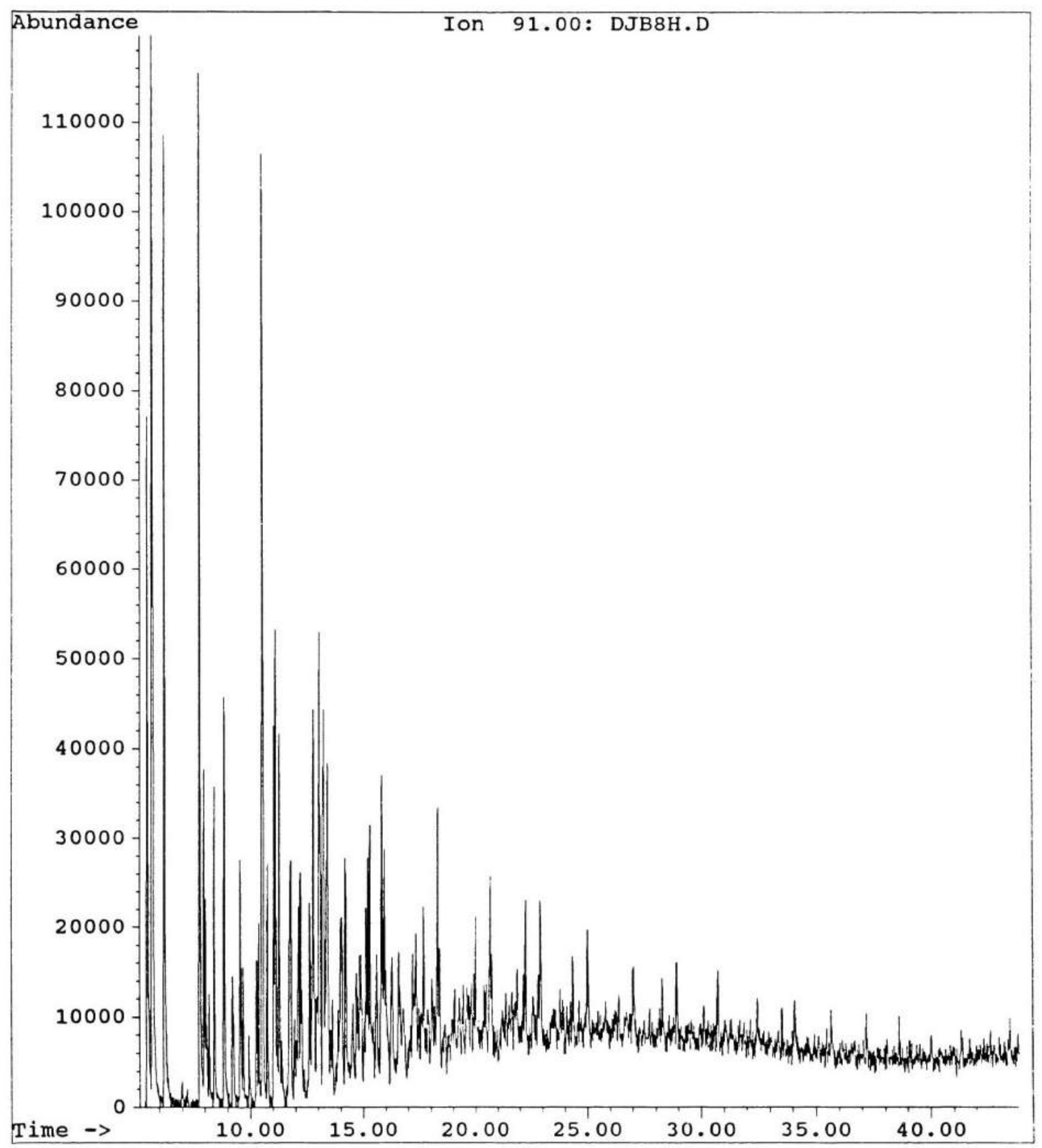


ARABIAN CRUDE 8 HRS WEATHERING

\begin{tabular}{|c|c|c|c|c|c|c|}
\hline Peak\# & Ret Time & Type & width & Area & Start Time & End Time \\
\hline 1 & 5.494 & $B V$ & 0.062 & 2933544 & 5.432 & 5.647 \\
\hline 2 & 5.709 & VV & 0.073 & 5675458 & 5.647 & 6.036 \\
\hline 3 & 6.232 & VV & 0.060 & 4144450 & 6.169 & 6.477 \\
\hline 4 & 7.776 & BV & 0.050 & 3625473 & 7.655 & 7.918 \\
\hline 5 & 7.970 & VV & 0.048 & 1126978 & 7.918 & 8.019 \\
\hline 6 & 8.054 & VV & 0.051 & 764822 & 8.019 & 8.152 \\
\hline 7 & 8.193 & VV & 0.045 & 372194 & 8.152 & 8.290 \\
\hline 8 & 8.422 & VV & 0.048 & 1135759 & 8.349 & 8.533 \\
\hline 9 & 8.849 & PV & 0.052 & 1536692 & 8.761 & 9.032 \\
\hline 10 & 9.214 & PV & 0.069 & 681563 & 9.156 & 9.365 \\
\hline 11 & 9.561 & BV & 0.061 & 973875 & 9.495 & 9.635 \\
\hline 12 & 9.683 & VV & 0.046 & 427914 & 9.635 & 9.766 \\
\hline 13 & 10.277 & PV & 0.046 & 514518 & 10.185 & 10.329 \\
\hline 14 & 10.373 & VV & 0.050 & 571273 & 10.329 & 10.449 \\
\hline 15 & 10.520 & VV & 0.063 & 4382843 & 10.449 & 10.692 \\
\hline 16 & 10.754 & VV & 0.049 & 837855 & 10.692 & 10.854 \\
\hline 17 & 11.032 & PV & 0.042 & 1091868 & 10.962 & 11.070 \\
\hline 18 & 11.107 & VV & 0.049 & 1616650 & 11.070 & 11.217 \\
\hline 19 & 11.275 & VV & 0.053 & 1340466 & 11.217 & 11.354 \\
\hline 20 & 11.783 & VV & 0.087 & 1690958 & 11.628 & 11.889 \\
\hline 21 & 11.958 & VV & 0.051 & 311231 & 11.889 & 11.995 \\
\hline 22 & 12.138 & VV & 0.050 & 737932 & 12.080 & 12.177 \\
\hline 23 & 12.216 & VV & 0.048 & 777894 & 12.177 & 12.256 \\
\hline 24 & 12.279 & VV & 0.048 & 362868 & 12.256 & 12.333 \\
\hline 25 & 12.620 & VV & 0.048 & 708240 & 12.480 & 12.666 \\
\hline 26 & 12.787 & VV & 0.068 & 2050190 & 12.666 & 12.888 \\
\hline 27 & 12.931 & VV & 0.083 & 569692 & 12.888 & 12.990 \\
\hline 28 & 13.035 & VV & 0.064 & 2316884 & 12.990 & 13.144 \\
\hline 29 & 13.198 & VV & 0.038 & 834034 & 13.144 & 13.214 \\
\hline 30 & 13.244 & VV & 0.054 & 1401168 & 13.214 & 13.294 \\
\hline 31 & 13.403 & VV & 0.080 & 2001944 & 13.338 & 13.501 \\
\hline 32 & 13.874 & VV & 0.056 & 361328 & 13.820 & 13.922 \\
\hline 33 & 14.034 & VV & 0.097 & 1451437 & 13.922 & 14.140 \\
\hline 34 & 14.207 & VV & 0.072 & 1210614 & 14.140 & 14.344 \\
\hline 35 & 14.541 & VV & 0.090 & 658134 & 14.410 & 14.630 \\
\hline 36 & 14.693 & VV & 0.042 & 356171 & 14.630 & 14.721 \\
\hline 37 & 14.847 & VV & 0.067 & 763444 & 14.797 & 14.913 \\
\hline 38 & 15.121 & VV & 0.053 & 680019 & 15.073 & 15.163 \\
\hline 39 & 15.199 & VV & 0.044 & 724141 & 15.163 & 15.241 \\
\hline 40 & 15.285 & VV & 0.058 & 1109191 & 15.241 & 15.391 \\
\hline 41 & 15.598 & VV & 0.068 & 759433 & 15.501 & 15.654 \\
\hline 42 & 15.816 & VV & 0.065 & 1536583 & 15.713 & 15.884 \\
\hline 43 & 15.936 & VV & 0.052 & 952393 & 15.884 & 15.983 \\
\hline 44 & 16.279 & PV & 0.079 & 821430 & 16.165 & 16.341 \\
\hline 45 & 16.578 & VV & 0.066 & 657665 & 16.442 & 16.616 \\
\hline 46 & 17.194 & VV & 0.057 & 480897 & 17.134 & 17.226 \\
\hline 47 & 17.342 & VV & 0.067 & 623136 & 17.292 & 17.411 \\
\hline 48 & 17.690 & VV & 0.048 & 629283 & 17.630 & 17.743 \\
\hline 49 & 18.055 & VV & 0.081 & 605278 & 17.948 & 18.136 \\
\hline 50 & 18.310 & VV & 0.056 & 1129495 & 18.207 & 18.354 \\
\hline 51 & 18.387 & VV & 0.048 & 448155 & 18.354 & 18.465 \\
\hline 52 & 20.015 & VV & 0.055 & 544550 & 19.966 & 20.094 \\
\hline 53 & 20.667 & VV & 0.066 & 848252 & 20.545 & 20.704 \\
\hline 54 & 20.733 & VV & 0.051 & 333880 & 20.704 & 20.784 \\
\hline 55 & 22.247 & VV & 0.044 & 438320 & 22.184 & 22.311 \\
\hline 56 & 22.896 & VV & 0.064 & 776089 & 22.859 & 23.017 \\
\hline
\end{tabular}


File:

operator:

Date Acquired: 21 Jul 94

Method File: JAFFE.M

Sample Name:

Misc Info:

ALS vial:

1

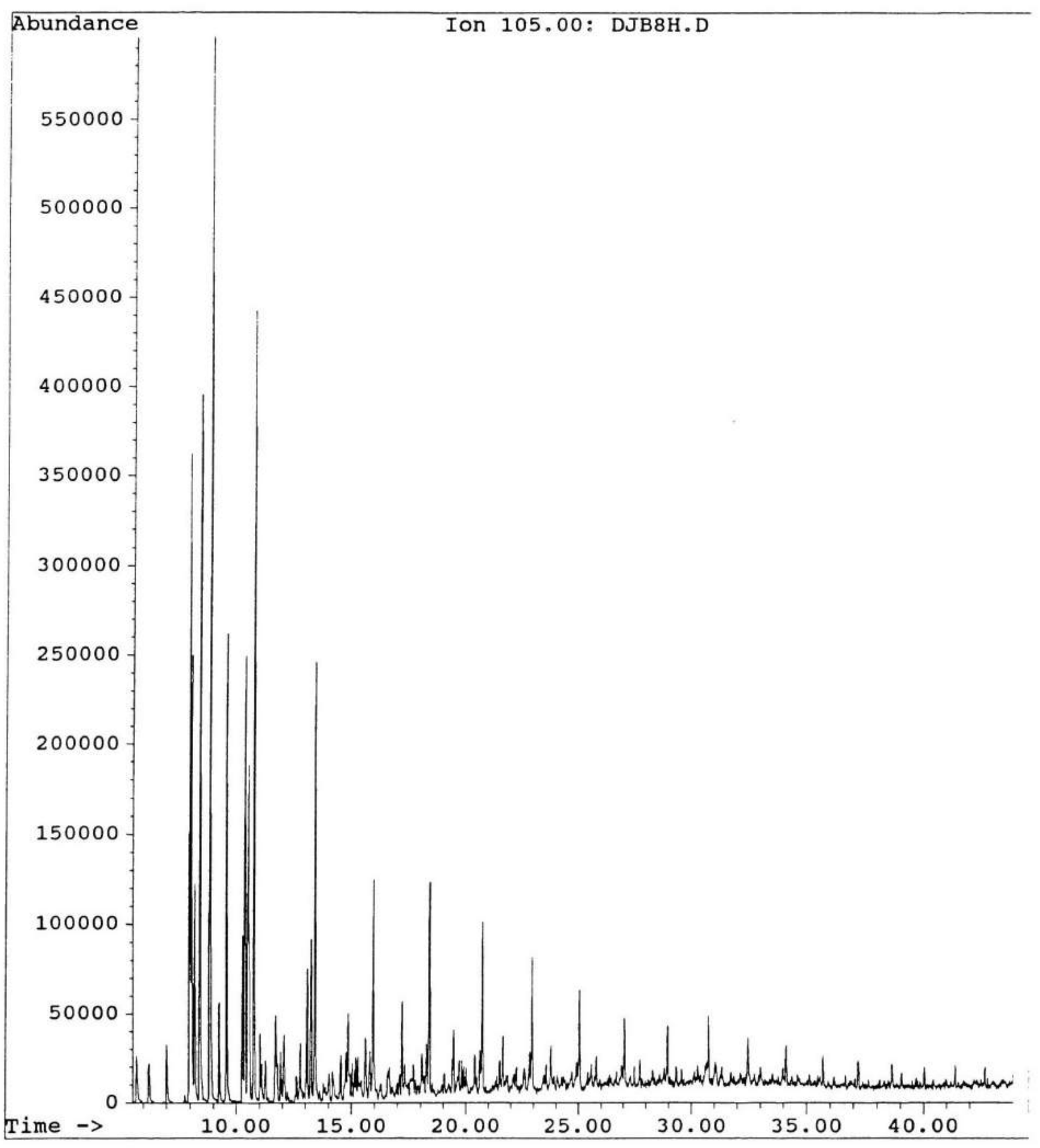


Ion 105.00: DJB8H.D

ARABIAN CRUDE 8 HRS WEATHERING

\begin{tabular}{|c|c|c|c|c|c|c|}
\hline Peak\# & Ret Time & Type & width & Area & Start Time & End Time \\
\hline 1 & 5.702 & $\mathrm{BB}$ & 0.064 & 1179983 & 5.643 & 5.882 \\
\hline 2 & 7.002 & BB & 0.050 & 1059485 & 6.956 & 7.147 \\
\hline 3 & 7.971 & BV & 0.046 & 10647658 & 7.895 & 8.018 \\
\hline 4 & 8.055 & VV & 0.051 & 8094407 & 8.018 & 8.145 \\
\hline 5 & 8.195 & VV & 0.048 & 3908351 & 8.145 & 8.355 \\
\hline 6 & 8.421 & VB & 0.046 & 11540173 & 8.355 & 8.671 \\
\hline 7 & 8.850 & BB & 0.048 & 18142558 & 8.767 & 9.121 \\
\hline 8 & 9.260 & BB & 0.043 & 1623405 & 9.198 & 9.409 \\
\hline 9 & 9.571 & BB & 0.047 & 7791088 & 9.486 & 9.802 \\
\hline 10 & 10.280 & BV & 0.042 & 2387688 & 10.214 & 10.324 \\
\hline 11 & 10.371 & VV & 0.046 & 7002869 & 10.324 & 10.453 \\
\hline 12 & 10.509 & VV & 0.060 & 7719063 & 10.453 & 10.687 \\
\hline 13 & 10.755 & VV & 0.045 & 12534963 & 10.687 & 10.981 \\
\hline 14 & 11.035 & PV & 0.038 & 782894 & 10.981 & 11.077 \\
\hline 15 & 11.713 & BV & 0.086 & 2770120 & 11.613 & 11.874 \\
\hline 16 & 11.929 & VV & 0.044 & 757909 & 11.874 & 11.995 \\
\hline 17 & 12.066 & VV & 0.055 & 1369026 & 11.995 & 12.177 \\
\hline 18 & 12.790 & VV & 0.058 & 1302353 & 12.677 & 12.890 \\
\hline 19 & 13.080 & VV & 0.051 & 2376711 & 12.989 & 13.162 \\
\hline 20 & 13.247 & VV & 0.046 & 2707947 & 13.162 & 13.333 \\
\hline 21 & 13.412 & VV & 0.050 & 7608946 & 13.333 & 13.590 \\
\hline 22 & 14.541 & VV & 0.051 & 909215 & 14.411 & 14.627 \\
\hline 23 & 14.770 & VV & 0.062 & 1119983 & 14.627 & 14.804 \\
\hline 24 & 14.849 & VV & 0.069 & 2239671 & 14.804 & 14.977 \\
\hline 25 & 15.608 & VV & 0.058 & 1414294 & 15.504 & 15.712 \\
\hline 26 & 15.804 & VV & 0.055 & 1053652 & 15.712 & 15.855 \\
\hline 27 & 15.938 & VV & 0.053 & 4423403 & 15.855 & 16.165 \\
\hline 28 & 17.193 & VV & 0.048 & 1659905 & 17.134 & 17.278 \\
\hline 29 & 18.266 & VV & 0.047 & 800779 & 18.210 & 18.319 \\
\hline 30 & 18.389 & VV & 0.047 & 3577655 & 18.319 & 18.473 \\
\hline 31 & 19.454 & VV & 0.063 & 1464942 & 19.334 & 19.511 \\
\hline 32 & 20.736 & VV & 0.046 & 2812152 & 20.659 & 20.857 \\
\hline 33 & 21.650 & VV & 0.048 & 890574 & 21.571 & 21.703 \\
\hline 34 & 22.946 & VV & 0.048 & 2375953 & 22.859 & 23.084 \\
\hline 35 & 25.050 & VV & 0.052 & 1893373 & 24.962 & 25.163 \\
\hline 36 & 27.047 & VV & 0.053 & 1219818 & 26.987 & 27.167 \\
\hline 37 & 28.948 & VV & 0.046 & 1026681 & 28.845 & 29.000 \\
\hline 38 & 30.758 & VV & 0.056 & 945031 & 30.715 & 30.816 \\
\hline 39 & 32.474 & PV & 0.041 & 519511 & 32.420 & 32.527 \\
\hline
\end{tabular}


File:

Operator:

Date Acquired: $21 \mathrm{Jul} 94$

Method File:

Sample Name:

Misc Info:

ALS vial:

BOYE

JAFFE.M
C: $\backslash$ CHEMPC \DATA $\backslash D J B 8 H . D$

9:25 pm

ARABIAN CRUDE 8 HRS WEATHERING

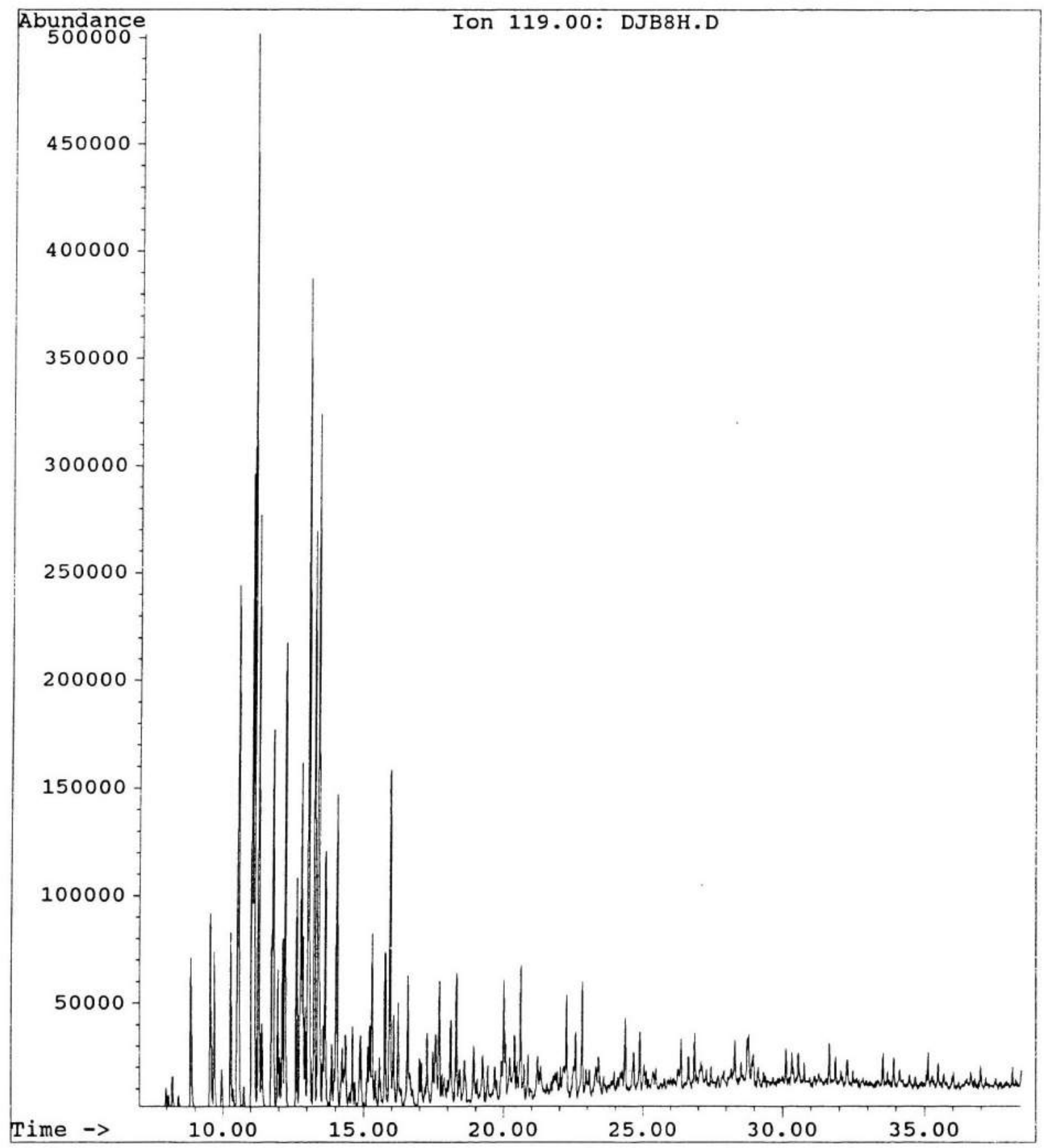


ARABIAN CRUDE 8 HRS WEATHERING

\begin{tabular}{|c|c|c|c|c|c|c|}
\hline Peak\# & Ret Time & Type & Width & Area & Start Time & End Time \\
\hline 1 & 8.851 & $\mathrm{BB}$ & 0.049 & 2250765 & 8.786 & 9.006 \\
\hline 2 & 9.547 & BV & 0.052 & 3082762 & 9.486 & 9.635 \\
\hline 3 & 9.686 & VB & 0.046 & 2052123 & 9.635 & 9.840 \\
\hline 4 & 10.280 & BV & 0.043 & 2274152 & 10.214 & 10.343 \\
\hline 5 & 10.546 & PV & 0.055 & 8569139 & 10.456 & 10.683 \\
\hline 6 & 11.035 & PV & 0.041 & 7817429 & 10.842 & 11.070 \\
\hline 7 & 11.108 & VV & 0.044 & 13600153 & 11.070 & 11.208 \\
\hline 8 & 11.273 & PV & 0.044 & 7706465 & 11.208 & 11.348 \\
\hline 9 & 11.785 & BV & 0.064 & 7672716 & 11.661 & 11.895 \\
\hline 10 & 11.958 & VV & 0.039 & 1531504 & 11.895 & 12.004 \\
\hline 11 & 12.126 & VV & 0.045 & 2247936 & 12.076 & 12.169 \\
\hline 12 & 12.217 & VB & 0.044 & 5912477 & 12.169 & 12.361 \\
\hline 13 & 12.621 & BV & 0.044 & 2989571 & 12.562 & 12.687 \\
\hline 14 & 12.786 & vV & 0.050 & 5246381 & 12.687 & 12.824 \\
\hline 15 & 12.849 & VV & 0.039 & 2066165 & 12.824 & 12.891 \\
\hline 16 & 12.926 & VV & 0.043 & 1334422 & 12.891 & 12.973 \\
\hline 17 & 13.035 & $\mathrm{VV}$ & 0.051 & 12315303 & 12.973 & 13.174 \\
\hline 18 & 13.245 & VV & 0.047 & 8053777 & 13.174 & 13.318 \\
\hline 19 & 13.383 & vV & 0.051 & 10773465 & 13.318 & 13.509 \\
\hline 20 & 13.562 & vV & 0.042 & 1036977 & 13.509 & 13.591 \\
\hline 21 & 13.633 & VV & 0.045 & 3427866 & 13.591 & 13.743 \\
\hline 22 & 14.041 & VV & 0.049 & 4595181 & 13.986 & 14.142 \\
\hline 23 & 14.604 & VV & 0.042 & 936320 & 14.534 & 14.655 \\
\hline 24 & 15.290 & vV & 0.044 & 2102928 & 15.247 & 15.374 \\
\hline 25 & 15.757 & BV & 0.044 & 1990924 & 15.705 & 15.849 \\
\hline 26 & 15.929 & VV & 0.047 & 4645429 & 15.849 & 16.009 \\
\hline 27 & 16.066 & VV & 0.060 & 1313867 & 16.009 & 16.151 \\
\hline 28 & 16.222 & PV & 0.046 & 1302924 & 16.151 & 16.320 \\
\hline 29 & 16.574 & BV & 0.055 & 2134501 & 16.453 & 16.711 \\
\hline 30 & 17.689 & VV & 0.045 & 1613889 & 17.634 & 17.742 \\
\hline 31 & 18.099 & VB & 0.059 & 1422582 & 18.017 & 18.216 \\
\hline 32 & 18.305 & BV & 0.046 & 1706382 & 18.226 & 18.361 \\
\hline 33 & 20.013 & VV & 0.055 & 1521831 & 19.957 & 20.051 \\
\hline 34 & 20.606 & VV & 0.041 & 1531509 & 20.525 & 20.676 \\
\hline 35 & 22.247 & vV & 0.048 & 1468217 & 22.190 & 22.366 \\
\hline 36 & 22.810 & PV & 0.052 & 1742588 & 22.647 & 22.868 \\
\hline
\end{tabular}


File:

Operator:

Date Acquired:

Method File:

Sample Name:

Misc Info:

ALS vial:
C: \CHEMPC \DATA \DJB8H.D

BOYE

21 Jul 94 9:25 pm

JAFFE.M

ARABIAN CRUDE 8 HRS WEATHERING

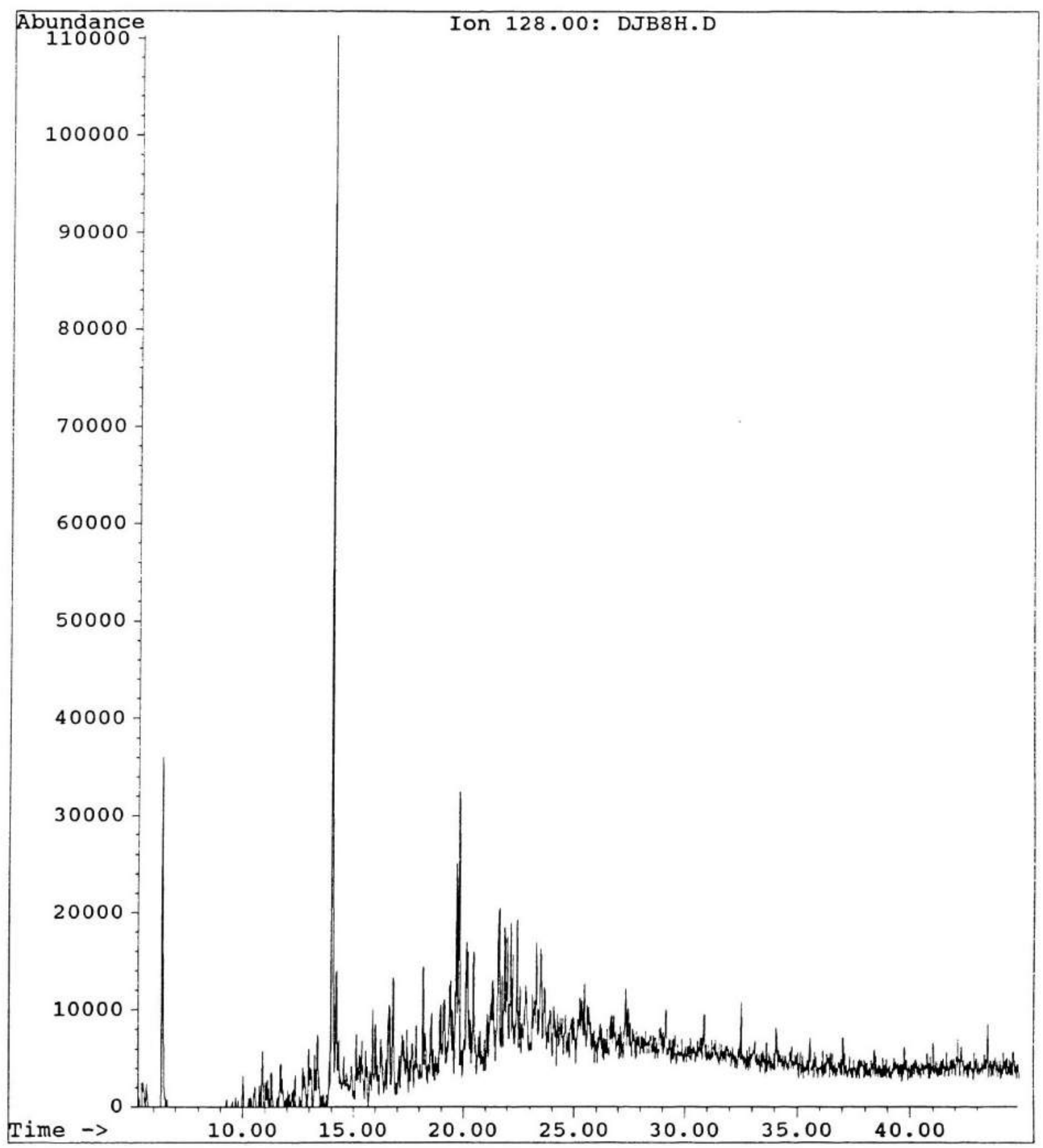


ARABIAN CRUDE 8 HRS WEATHERING

$\begin{array}{crlrrrr}\text { Peak\# } & \text { Ret Time } & \text { Type } & \text { Width } & \text { Area } & \text { Start Time } & \text { End Time } \\ 1 & 6.389 & \text { BB } & 0.060 & 1379940 & 6.333 & 6.582 \\ 2 & 13.996 & \text { VV } & 0.055 & 3981706 & 13.932 & 14.138 \\ 3 & 14.209 & \text { VV } & 0.055 & 375417 & 14.138 & 14.268 \\ 4 & 16.807 & \text { VV } & 0.068 & 546754 & 16.701 & 16.951 \\ 5 & 18.172 & \text { BV } & 0.046 & 310604 & 18.072 & 18.221 \\ 6 & 19.680 & \text { VV } & 0.065 & 1006070 & 19.571 & 19.732 \\ 7 & 19.792 & \text { VV } & 0.065 & 1129199 & 19.732 & 19.858 \\ 8 & 20.130 & \text { PV } & 0.085 & 672983 & 19.960 & 20.172 \\ 9 & 20.466 & \text { VV } & 0.065 & 547808 & 20.354 & 20.547 \\ 10 & 21.614 & \text { PV } & 0.078 & 898652 & 21.457 & 21.708 \\ 11 & 21.877 & \text { VV } & 0.057 & 457199 & 21.807 & 21.923 \\ 12 & 22.138 & \text { VV } & 0.074 & 512744 & 22.053 & 22.193 \\ 13 & 22.439 & \text { VV } & 0.058 & 494774 & 22.343 & 22.510\end{array}$


File:

Operator:

Date Acquired:

Method File:

Sample Name:

Misc Info:

ALS vial:
C: \CHEMPC \DATA \DJB8H.D

BOYE

21 Jul $94 \quad 9: 25 \mathrm{pm}$

JAFFE.M

1

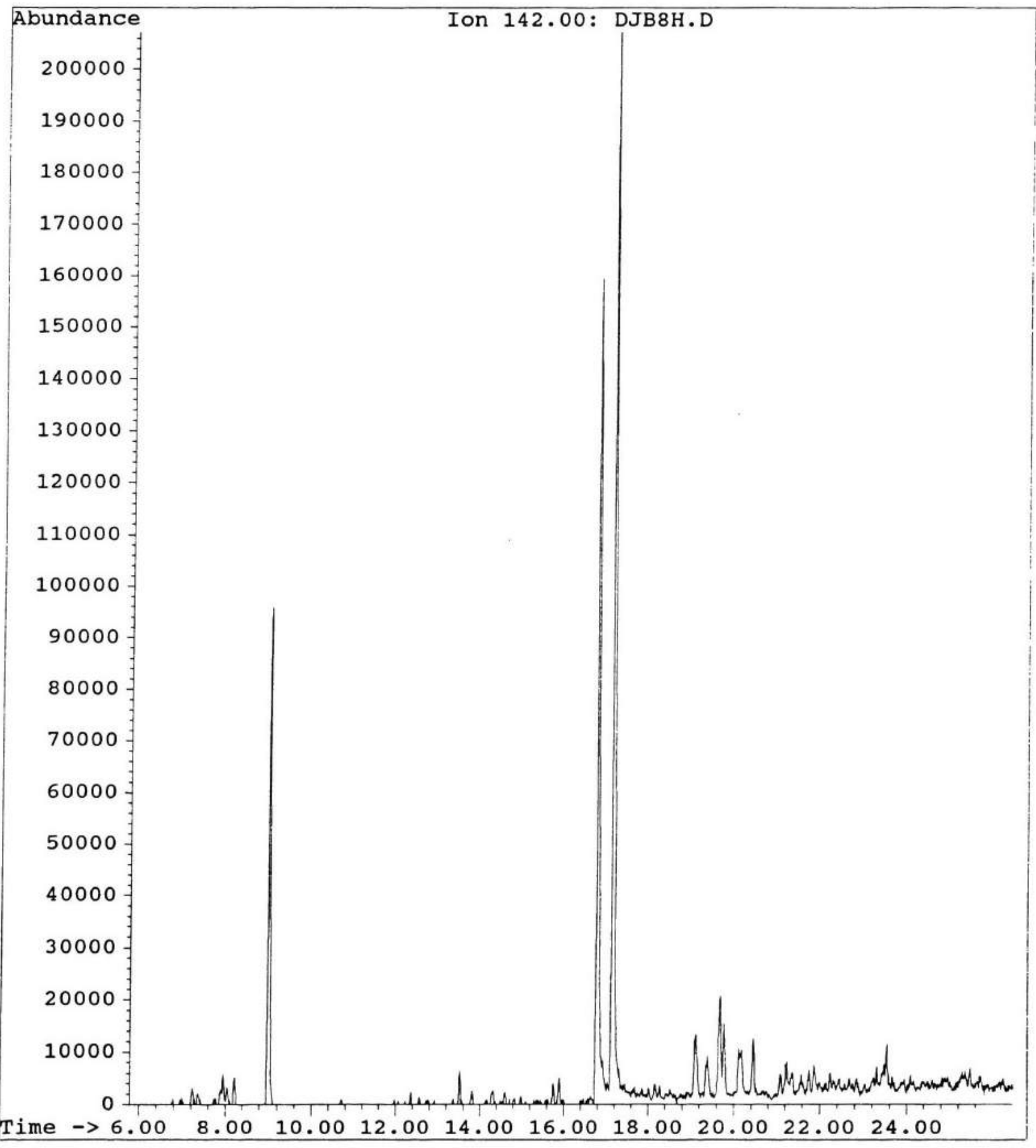


Ion $142.00:$ DJB8H.D

ARABIAN CRUDE 8 HRS WEATHERING

$\begin{array}{crlrrrr}\text { Peak\# } & \text { Ret Time } & \text { Type } & \text { Width } & \text { Area } & \text { Start Time } & \text { End Time } \\ 1 & 9.012 & \text { BB } & 0.049 & 2962320 & 8.930 & 9.112 \\ 2 & 16.785 & \text { PV } & 0.055 & 5716887 & 16.712 & 17.011 \\ 3 & 17.156 & \text { PV } & 0.054 & 7124162 & 17.076 & 17.306 \\ 4 & 19.127 & \text { BV } & 0.068 & 587808 & 18.992 & 19.240 \\ 5 & 19.684 & \text { PV } & 0.059 & 790073 & 19.570 & 19.739 \\ 6 & 19.781 & \text { VV } & 0.055 & 510338 & 19.739 & 19.931\end{array}$


File:

Operator:

Date Acquired:

Method File:

Sample Name:

Misc Info:

ALS vial:
C: \CHEMPC \DATA \DJB8H.D

BOYE

21 Jul $94 \quad 9: 25 \mathrm{pm}$

JAFFE.M

ARABIAN CRUDE 8 HRS WEATHERING

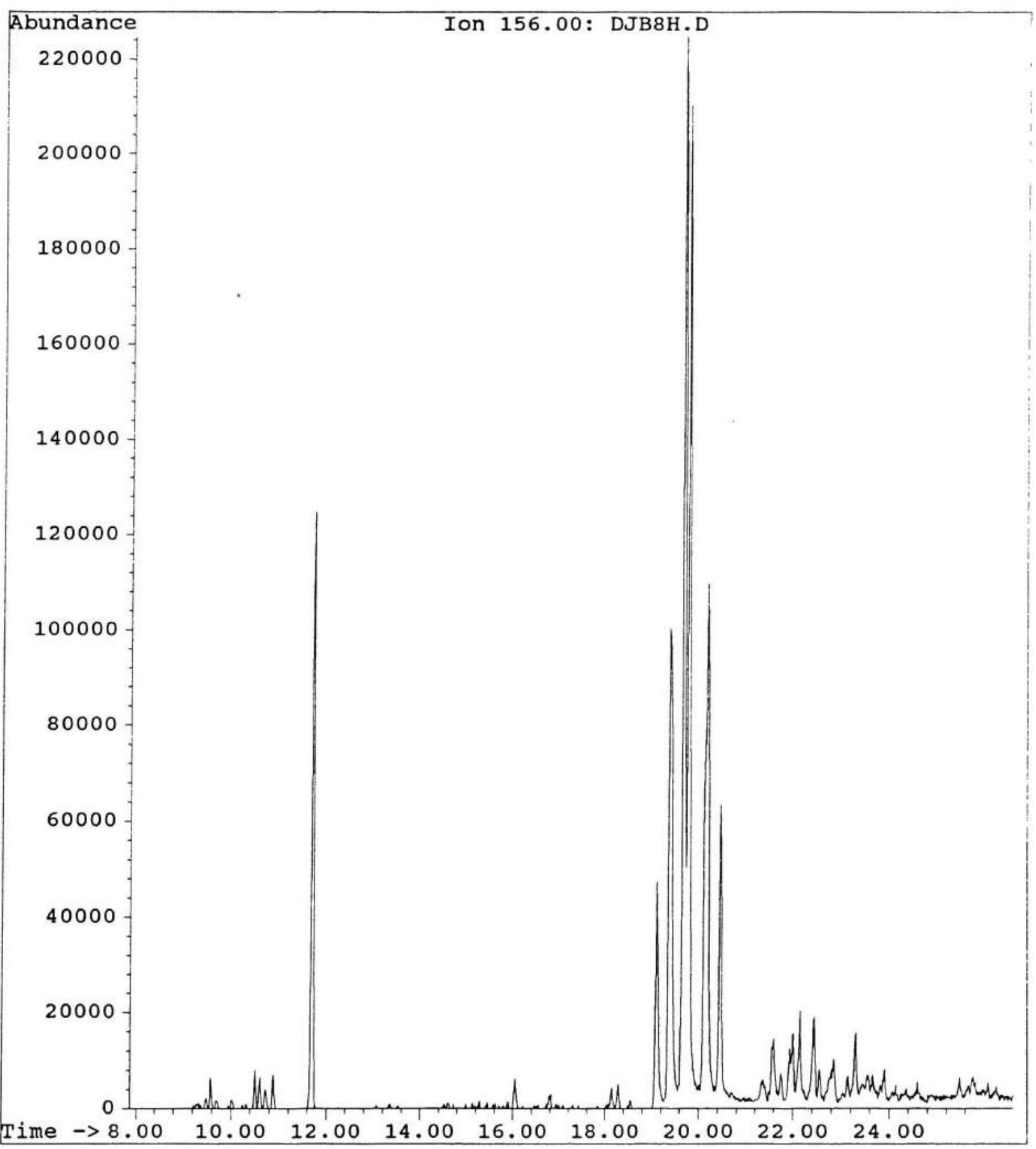


ARABIAN CRUDE 8 HRS WEATHERING

$\begin{array}{crlrrrr}\text { Peak\# } & \text { Ret Time } & \text { Type } & \text { Width } & \text { Area } & \text { Start Time } & \text { End Time } \\ 1 & 11.714 & \text { BB } & 0.059 & 3980771 & 11.613 & 11.786 \\ 2 & 19.111 & \text { BV } & 0.062 & 1982291 & 19.011 & 19.266 \\ 3 & 19.392 & \text { VV } & 0.094 & 5889575 & 19.266 & 19.559 \\ 4 & 19.682 & \text { VV } & 0.059 & 8586680 & 19.559 & 19.734 \\ 5 & 19.786 & \text { VV } & 0.056 & 7691937 & 19.734 & 19.979 \\ 6 & 20.187 & \text { VV } & 0.082 & 6381737 & 19.979 & 20.325 \\ 7 & 20.462 & \text { VV } & 0.058 & 2302703 & 20.361 & 20.663\end{array}$


File:

C: \CHEMPC \DATA \DJB8H.D

Operator:

BOYE

Date Acquired: $21 \mathrm{Jul} 94 \quad 9: 25 \mathrm{pm}$

Method File: JAFFE.M

Sample Name:

ARABIAN CRUDE 8 HRS WEATHERING

Misc Info:

ALS vial:

1

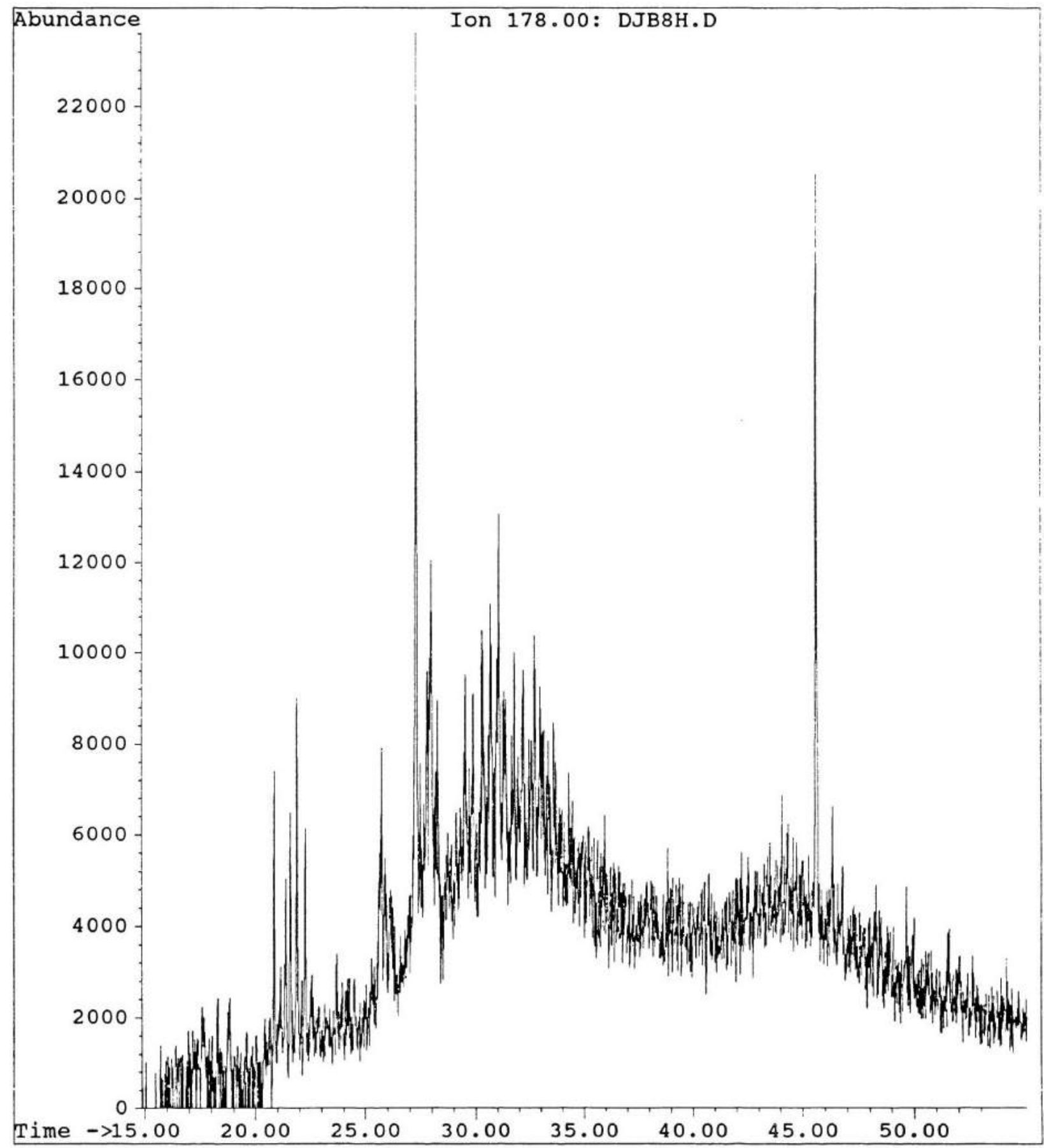


Ion $178.00:$ DJB8H.D

ARABIAN CRUDE 8 HRS WEATHERING

$\begin{array}{crlrr}\text { Peak\# } & \text { Ret Time } & \text { Type } & \text { Width } & \text { Area } \\ 1 & 20.849 & \text { PV } & 0.060 & 238342 \\ 2 & 21.571 & \text { PV } & 0.082 & 247812 \\ 3 & 21.876 & \text { VV } & 0.064 & 353750 \\ 4 & 22.252 & \text { PV } & 0.068 & 220982 \\ 5 & 25.728 & \text { VV } & 0.050 & 168929 \\ 6 & 27.264 & \text { VV } & 0.072 & 1095311 \\ 7 & 27.776 & \text { VV } & 0.081 & 409843 \\ 8 & 27.882 & \text { VV } & 0.056 & 257576 \\ 9 & 27.956 & \text { VV } & 0.084 & 524667 \\ 10 & 28.236 & \text { VV } & 0.076 & 357629 \\ 11 & 29.517 & \text { VV } & 0.065 & 206497 \\ 12 & 30.275 & \text { VV } & 0.080 & 332877 \\ 13 & 30.665 & \text { VV } & 0.079 & 354159 \\ 14 & 30.962 & \text { PV } & 0.066 & 215155 \\ 15 & 31.051 & \text { VV } & 0.068 & 367375 \\ 16 & 31.765 & \text { VV } & 0.039 & 88243 \\ 17 & 32.702 & \text { VV } & 0.078 & 229809 \\ 18 & 33.573 & \text { PV } & 0.062 & 132615 \\ 19 & 45.622 & \text { VV } & 0.069 & 746321\end{array}$

Start Time 20.737

21.479

21.791

22.124

25.669

27.164

27.659

27.825

27.902

28.199

29.412

30.242

30.598

30.875

31.000

31.720

32.636

33.477

45.515
End Time 20.947

21.678

22.036

22.309

25.804

27.343

27.825

27.902

28.061

28.398

29.548

30.490

30.774

31.000

31.171

31.799

32.812

33.654

45.714 
File:

Operator:

Date Acquired: 21 Jul 94

Method File: JAFFE.M

Sample Name:

Misc Info:

ALS vial:

BOYE
C: \CHEMPC \DATA \DJB8H.D

$.25 \mathrm{pm}$

ARABIAN CRUDE 8 HRS WEATHERING

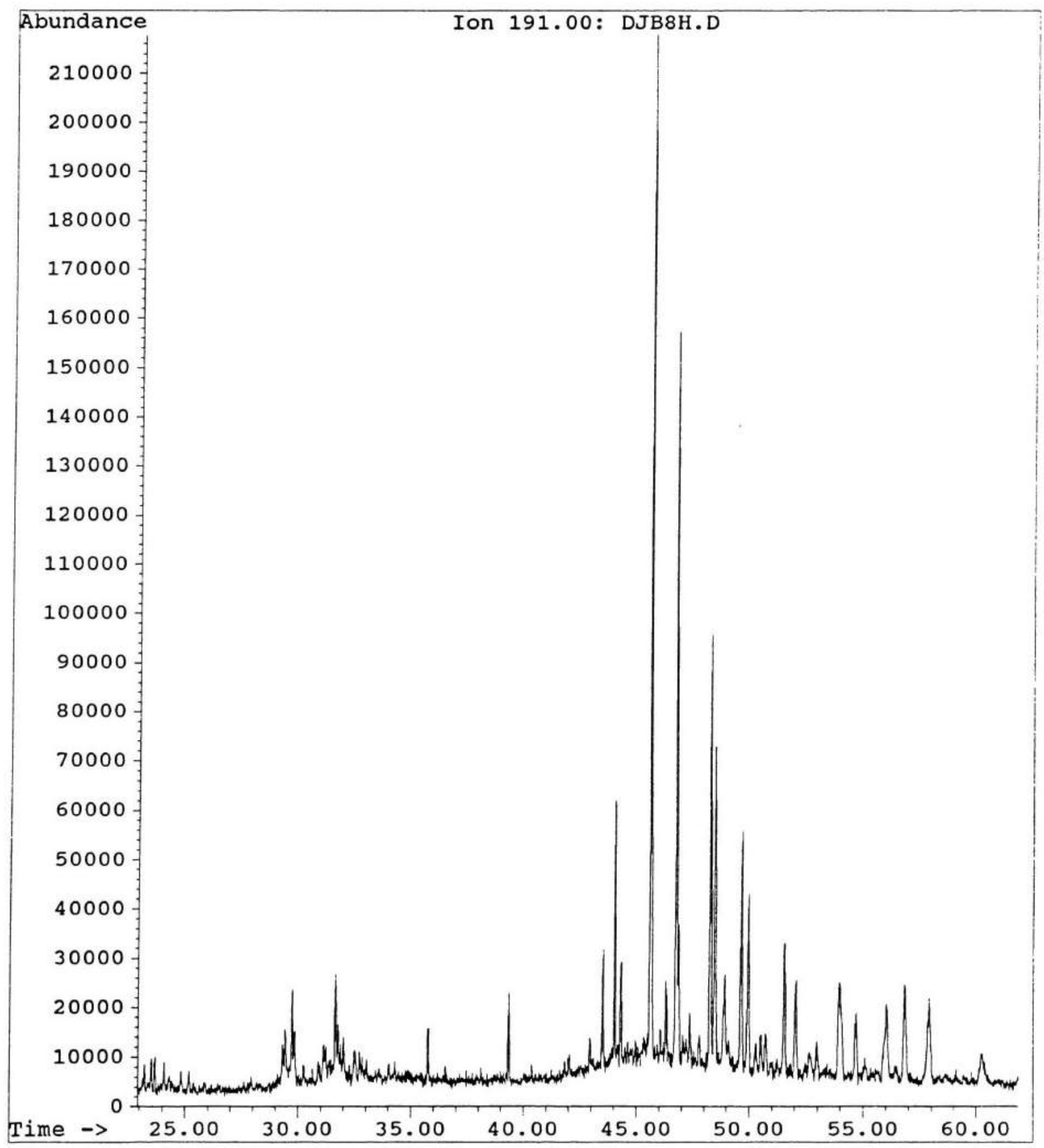


ARABIAN CRUDE 8 HRS WEATHERING

$\begin{array}{crlrr}\text { Peak\# } & \text { Ret Time } & \text { Type } & \text { Width } & \text { Area } \\ 1 & 29.736 & \text { VV } & 0.062 & 794018 \\ 2 & 31.645 & \text { PV } & 0.057 & 662326 \\ 3 & 39.361 & \text { VV } & 0.055 & 572520 \\ 4 & 43.514 & \text { PV } & 0.057 & 864309 \\ 5 & 44.037 & \text { VV } & 0.050 & 1757105 \\ 6 & 44.311 & \text { VV } & 0.057 & 758744 \\ 7 & 45.620 & \text { VV } & 0.061 & 8529813 \\ 8 & 46.314 & \text { VV } & 0.053 & 570499 \\ 9 & 46.760 & \text { VV } & 0.063 & 6093274 \\ 10 & 46.862 & \text { VV } & 0.062 & 1033944 \\ 11 & 48.283 & \text { BV } & 0.066 & 3894831 \\ 12 & 48.480 & \text { VV } & 0.076 & 3176491 \\ 13 & 48.925 & \text { VV } & 0.113 & 1574577 \\ 14 & 49.657 & \text { VV } & 0.075 & 2431387 \\ 15 & 49.963 & \text { VV } & 0.071 & 1851716 \\ 16 & 51.547 & \text { VV } & 0.084 & 1636659 \\ 17 & 52.046 & \text { VV } & 0.082 & 1173324 \\ 18 & 53.954 & \text { BV } & 0.136 & 2025042 \\ 19 & 56.031 & \text { BV } & 0.147 & 1382194 \\ 20 & 56.841 & \text { BV } & 0.112 & 1572103 \\ 21 & 57.909 & \text { PV } & 0.143 & 1863628\end{array}$

$\begin{array}{rr}\text { Start Time } & \text { End Time } \\ 29.601 & 29.794 \\ 31.566 & 31.711 \\ 39.298 & 39.491 \\ 43.352 & 43.650 \\ 43.965 & 44.110 \\ 44.229 & 44.431 \\ 45.434 & 45.783 \\ 46.215 & 46.384 \\ 46.613 & 46.829 \\ 46.829 & 46.965 \\ 48.011 & 48.381 \\ 48.381 & 48.608 \\ 48.747 & 49.052 \\ 49.507 & 49.781 \\ 49.846 & 50.105 \\ 51.419 & 51.653 \\ 51.930 & 52.170 \\ 53.770 & 54.186 \\ 55.706 & 56.190 \\ 56.655 & 57.034 \\ 57.442 & 58.159\end{array}$


File:

operator:

Date Acquired:

Method File:

Sample Name:

Misc Info:

ALS vial:
C: \CHEMPC \DATA \DJB8H.D

BOYE

21 Jul $94 \quad 9: 25 \mathrm{pm}$

JAFFE.M

ARABIAN CRUDE 8 HRS WEATHERING

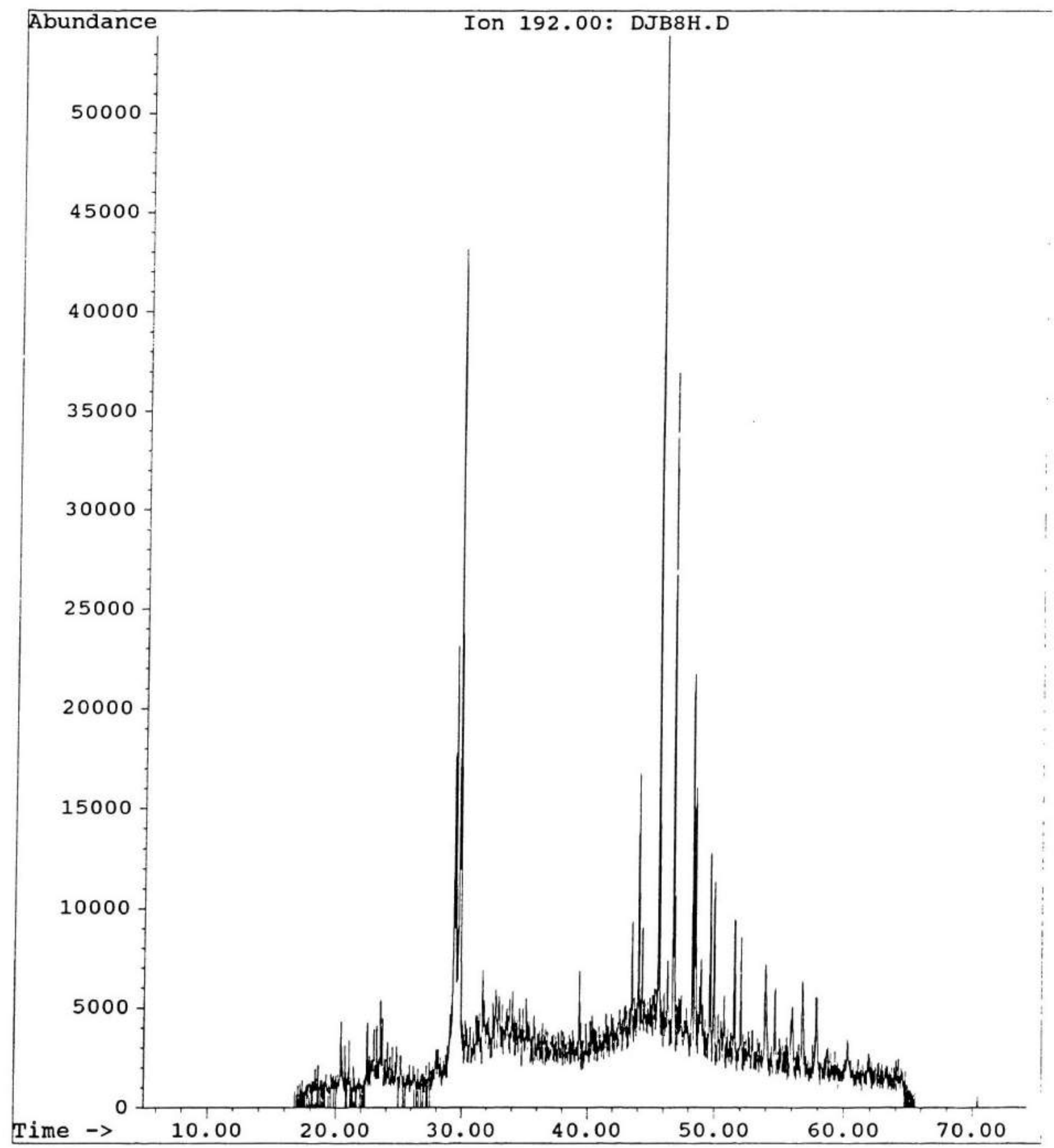


ARABIAN CRUDE 8 HRS WEATHERING

$\begin{array}{crlrrrr}\text { Peak\# } & \text { Ret Time } & \text { Type } & \text { Width } & \text { Area } & \text { Start Time } & \text { End Time } \\ 1 & 29.329 & \text { VV } & 0.079 & 840150 & 29.187 & 29.383 \\ 2 & 29.437 & \text { VV } & 0.068 & 938615 & 29.383 & 29.539 \\ 3 & 29.735 & \text { VV } & 0.065 & 1757802 & 29.609 & 29.796 \\ 4 & 29.842 & \text { VV } & 0.060 & 816603 & 29.796 & 29.940 \\ 5 & 44.041 & \text { PV } & 0.051 & 380762 & 43.954 & 44.104 \\ 6 & 45.622 & \text { VV } & 0.064 & 2072037 & 45.270 & 45.783 \\ 7 & 46.765 & \text { PV } & 0.056 & 1058449 & 46.650 & 46.839 \\ 8 & 48.283 & \text { PV } & 0.074 & 927652 & 48.075 & 48.366 \\ 9 & 48.474 & \text { VV } & 0.083 & 719682 & 48.366 & 48.605 \\ 10 & 49.654 & \text { BV } & 0.075 & 495258 & 49.419 & 49.737 \\ 11 & 49.954 & \text { BV } & 0.071 & 372179 & 49.841 & 50.035 \\ 12 & 51.551 & \text { PV } & 0.069 & 342548 & 51.442 & 51.644\end{array}$


File:

operator:

Date Acquired:

Method File:

Sample Name:

Misc Info:

ALS vial:
C: \CHEMPC \DATA \DJB8H.D

BOYE

21 Jul $94 \quad 9: 25 \mathrm{pm}$

JAFFE.M

1

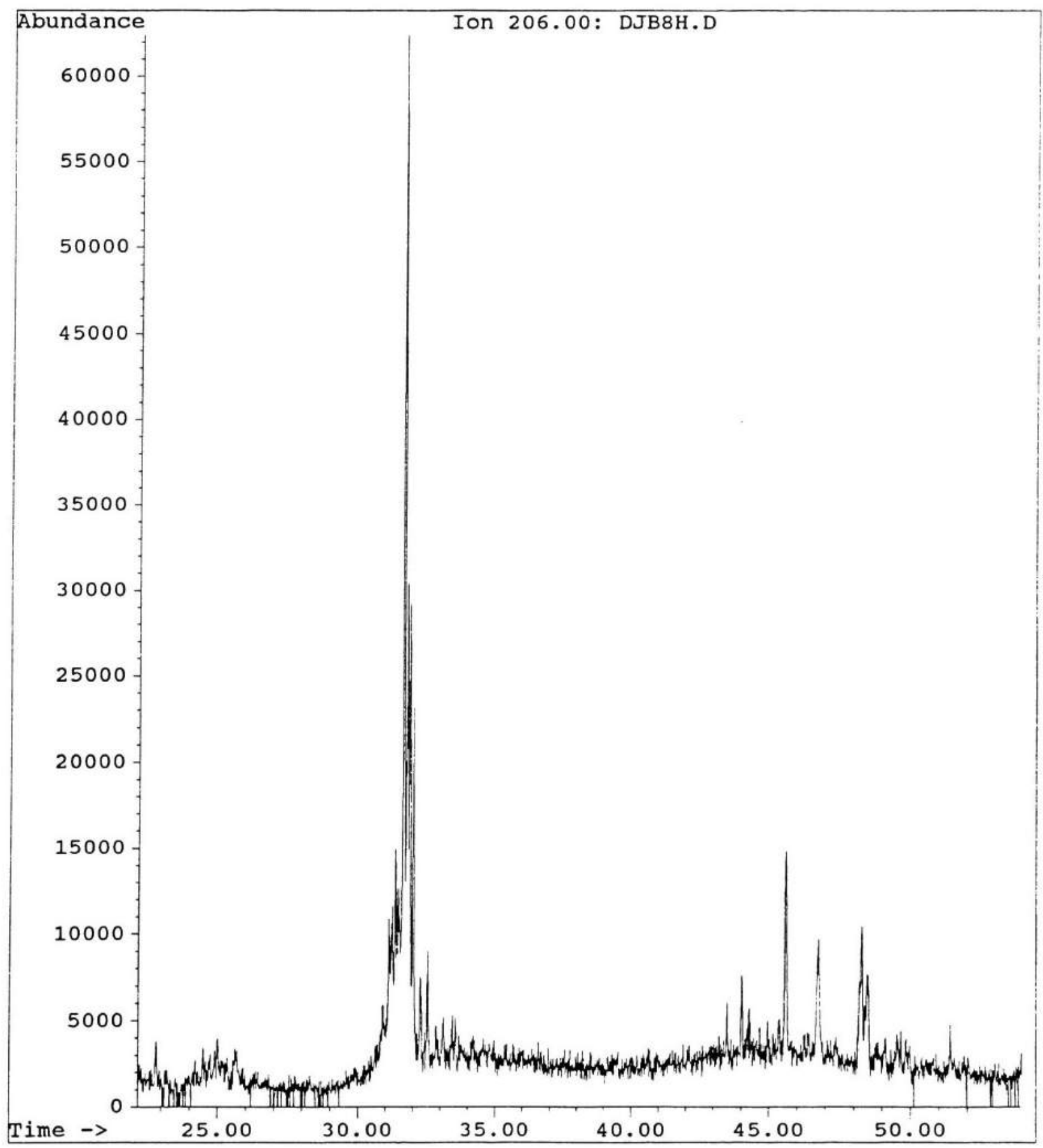


Ion $206.00:$ DJB8H.D

ARABIAN CRUDE 8 HRS WEATHERING

$\begin{array}{crlrrrr}\text { Peak\# } & \text { Ret Time } & \text { Type } & \text { Width } & \text { Area } & \text { Start Time } & \text { End Time } \\ 1 & 31.358 & \text { VV } & 0.068 & 556129 & 31.301 & 31.422 \\ 2 & 31.645 & \text { VV } & 0.066 & 2660053 & 31.546 & 31.716 \\ 3 & 31.771 & \text { VV } & 0.071 & 1284284 & 31.716 & 31.826 \\ 4 & 31.866 & \text { VV } & 0.058 & 1037096 & 31.826 & 31.942 \\ 5 & 32.000 & \text { VV } & 0.065 & 779205 & 31.942 & 32.131 \\ 6 & 45.602 & \text { PV } & 0.090 & 578647 & 45.504 & 45.719\end{array}$


File:

Operator:

Date Acquired: 21 Jul 94
Method File: JAFFE.M

Sample Name:

Misc Info:

ALS vial:

BOYE
C: \CHEMPC \DATA \DJB24H.D

$11: 20 \mathrm{pm}$

ARABIAN CRUDE 24 HRS WEATHERING

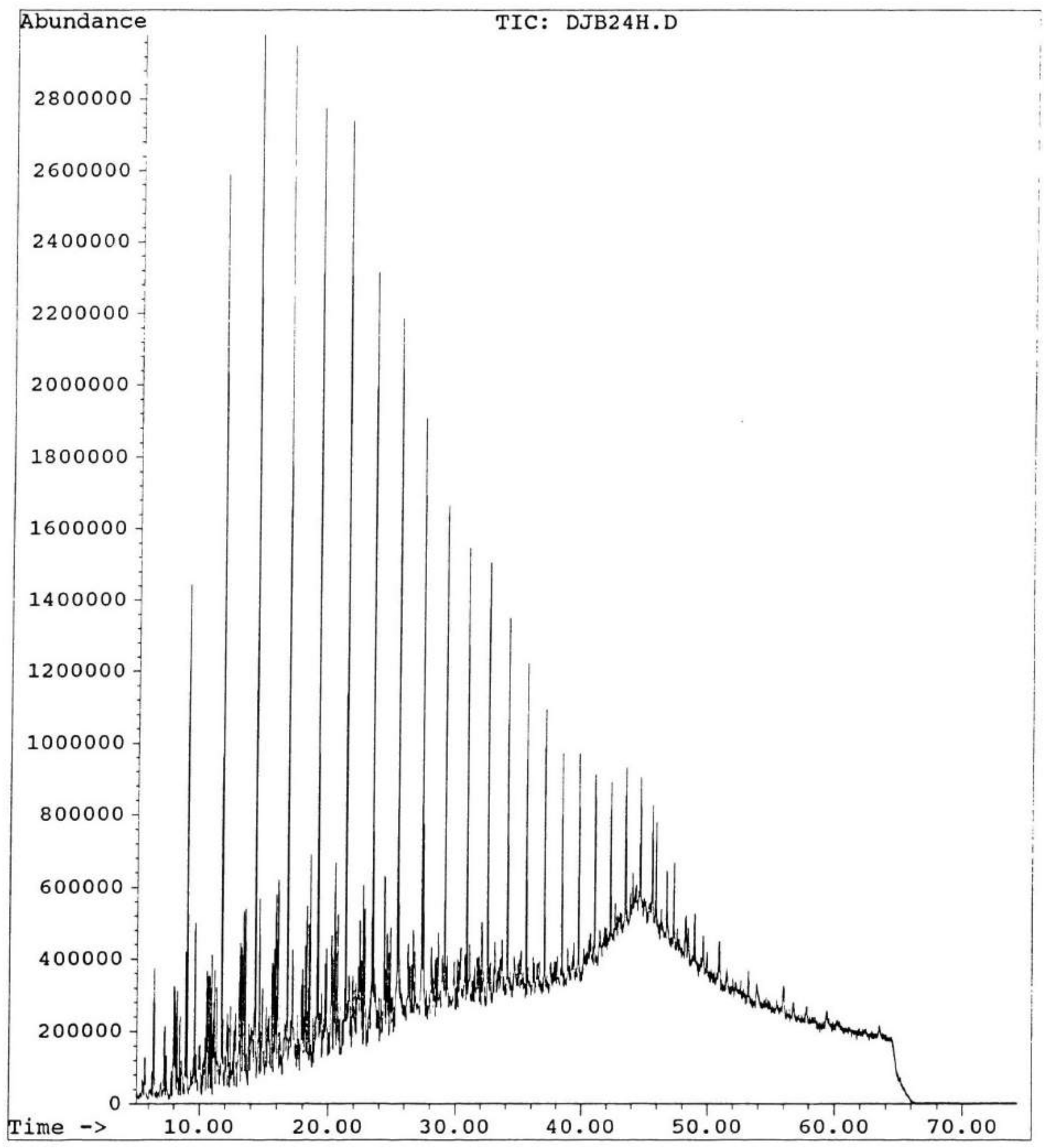


ARABIAN CRUDE 24 HRS WEATHERING

\begin{tabular}{|c|c|c|c|c|c|c|}
\hline Peak\# & Ret Time & Type & Width & Area & Start Time & End Time \\
\hline 1 & 5.705 & VV & 0.065 & 1457876 & 5.615 & 5.846 \\
\hline 2 & 6.394 & BB & 0.053 & 4466350 & 6.226 & 6.568 \\
\hline 3 & 7.241 & PV & 0.048 & 1660297 & 7.181 & 7.314 \\
\hline 4 & 7.381 & VV & 0.066 & 1335924 & 7.314 & 7.452 \\
\hline 5 & 7.954 & VV & 0.045 & 1979577 & 7.850 & 8.008 \\
\hline 6 & 8.050 & VV & 0.046 & 1814719 & 8.008 & 8.162 \\
\hline 7 & 8.218 & PV & 0.042 & 2036702 & 8.162 & 8.29 \\
\hline 8 & 9.002 & VV & 0.041 & 13980084 & 8.917 & 9.12 \\
\hline 9 & 9.580 & VV & 0.039 & 1746994 & 9.535 & 9.64 \\
\hline 10 & 10.505 & VV & 0.037 & 2098558 & 10.454 & 10.55 \\
\hline 11 & 10.602 & VV & 0.038 & 1306076 & 10.559 & 10.66 \\
\hline 12 & 10.724 & PV & 0.040 & 2187768 & 10.669 & 10.82 \\
\hline 13 & 10.886 & VV & 0.043 & 2816872 & 10.824 & 10.99 \\
\hline 14 & 11.690 & PV & 0.037 & 20135557 & 11.618 & 11.76 \\
\hline 15 & 12.069 & VV & 0.036 & 1034390 & 12.024 & 12.09 \\
\hline 16 & 12.365 & PV & 0.040 & 1067478 & 12.316 & 12.449 \\
\hline 17 & 13.076 & VV & 0.050 & 3626072 & 12.976 & 13.171 \\
\hline 18 & 13.222 & VV & 0.039 & 1208494 & 13.171 & 13.279 \\
\hline 19 & 13.360 & VV & 0.039 & 2595731 & 13.279 & 13.465 \\
\hline 20 & 13.522 & PV & 0.035 & 2832030 & 13.465 & 13.572 \\
\hline 21 & 14.301 & VV & 0.037 & 21801028 & 14.082 & 14.399 \\
\hline 22 & 14.607 & VV & 0.037 & 4139809 & 14.553 & 14.663 \\
\hline 23 & 15.574 & VV & 0.037 & 2068551 & 15.484 & 15.603 \\
\hline 24 & 15.622 & VV & 0.039 & 1142982 & 15.603 & 15.694 \\
\hline 25 & 15.749 & VV & 0.043 & 1375935 & 15.694 & 15.82 \\
\hline 26 & 15.888 & VV & 0.037 & 2485914 & 15.827 & 15.96 \\
\hline 27 & 16.053 & PV & 0.063 & 5526508 & 15.965 & 16.157 \\
\hline 28 & 16.786 & VV & 0.039 & 20410322 & 16.702 & 16.884 \\
\hline 29 & 17.161 & BV & 0.036 & 1429567 & 17.094 & 17.237 \\
\hline 30 & 17.947 & VV & 0.040 & 2102771 & 17.897 & 17.98 \\
\hline 31 & 18.018 & VV & 0.049 & 1077178 & 17.985 & 18.08 \\
\hline 32 & 18.141 & VV & 0.039 & 1367348 & 18.087 & 18.22 \\
\hline 33 & 18.280 & VV & 0.039 & 2332776 & 18.225 & 18.35 \\
\hline 34 & 18.434 & PV & 0.037 & 2350516 & 18.355 & 18.49 \\
\hline 35 & 18.542 & VV & 0.036 & 2954755 & 18.490 & 18.60 \\
\hline 36 & 19.135 & VV & 0.040 & 19615699 & 19.054 & 19.23 \\
\hline 37 & 20.207 & PV & 0.062 & 2334779 & 20.068 & 20.24 \\
\hline 38 & 20.274 & VV & 0.043 & 1075848 & 20.247 & 20.35 \\
\hline 39 & 20.410 & VV & 0.040 & 1032575 & 20.354 & 20.42 \\
\hline 40 & 20.459 & VV & 0.038 & 2164741 & 20.427 & 20.50 \\
\hline 41 & 20.547 & VV & 0.038 & 1903887 & 20.501 & 20.62 \\
\hline 42 & 20.694 & PV & 0.038 & 2128671 & 20.620 & 20.753 \\
\hline 43 & 21.356 & PV & 0.039 & 16260494 & 21.271 & 21.443 \\
\hline 44 & 22.347 & BV & 0.062 & 2394662 & 22.234 & 22.390 \\
\hline 45 & 22.427 & VV & 0.050 & 1310629 & 22.390 & 22.516 \\
\hline 46 & 22.687 & VV & 0.041 & 2025808 & 22.628 & 22.76 \\
\hline 47 & 22.832 & PV & 0.039 & 1697239 & 22.769 & $22.90^{\circ}$ \\
\hline 48 & 23.455 & PV & 0.040 & 14542144 & 23.309 & 23.52 \\
\hline 49 & 24.377 & PV & 0.069 & 5042016 & 24.226 & 24.43 \\
\hline 50 & 24.718 & VV & 0.043 & 1186413 & 24.668 & 24.77 \\
\hline 51 & 24.862 & VV & 0.042 & 1599633 & 24.777 & 24.917 \\
\hline 52 & 25.448 & PV & 0.045 & 14180160 & 25.238 & 25.552 \\
\hline 53 & 26.255 & VV & 0.053 & 1530892 & 26.130 & 26.29 \\
\hline 54 & 26.645 & PV & 0.044 & 1032522 & 26.567 & 26.73 \\
\hline 55 & 26.788 & PV & 0.037 & 878444 & 26.736 & 26.83 \\
\hline 56 & 27.341 & BV & 0.045 & 10841083 & 27.107 & 27.38 \\
\hline
\end{tabular}




$\begin{array}{lllllll}57 & 27.447 & \text { VV } & 0.063 & 4240125 & 27.389 & 27.611 \\ 58 & 28.085 & \text { PV } & 0.059 & 1413274 & 27.991 & 28.138 \\ 59 & 28.619 & \text { PV } & 0.038 & 809059 & 28.550 & 28.673 \\ 60 & 29.142 & \text { VV } & 0.039 & 8054195 & 29.071 & 29.204 \\ 61 & 30.860 & \text { PV } & 0.040 & 7046677 & 30.784 & 30.966 \\ 62 & 32.032 & \text { PV } & 0.047 & 1082829 & 31.971 & 32.152 \\ 63 & 32.502 & \text { PV } & 0.039 & 6351692 & 32.433 & 32.608 \\ 64 & 34.072 & \text { PV } & 0.041 & 5593166 & 33.995 & 34.129 \\ 65 & 35.576 & \text { PV } & 0.037 & 4424238 & 35.488 & 35.630 \\ 66 & 37.022 & \text { BV } & 0.040 & 3983263 & 36.930 & 37.076 \\ 67 & 38.407 & \text { BV } & 0.042 & 3413534 & 38.355 & 38.577 \\ 68 & 39.742 & \text { VV } & 0.043 & 3529389 & 39.618 & 39.923 \\ 69 & 41.029 & \text { PV } & 0.045 & 2684248 & 40.921 & 41.177 \\ 70 & 42.271 & \text { VV } & 0.041 & 2187197 & 42.211 & 42.450 \\ 71 & 43.467 & \text { PV } & 0.045 & 1830083 & 43.354 & 43.552 \\ 72 & 44.631 & \text { VV } & 0.048 & 1889411 & 44.571 & 44.747 \\ 73 & 45.879 & \text { VB } & 0.048 & 1565059 & 45.808 & 46.041 \\ 74 & 47.294 & \text { PV } & 0.052 & 1354488 & 47.191 & 47.434\end{array}$


File:

Operator:

Date Acquired: $21 \mathrm{Ju}$

Method File:

Sample Name:

Misc Info:

ALS vial:

BOYE
C: \CHEMPC \DATA \DJB24H.D

JAFFE.M

$11: 20 \mathrm{pm}$

ARABIAN CRUDE 24 HRS WEATHERING

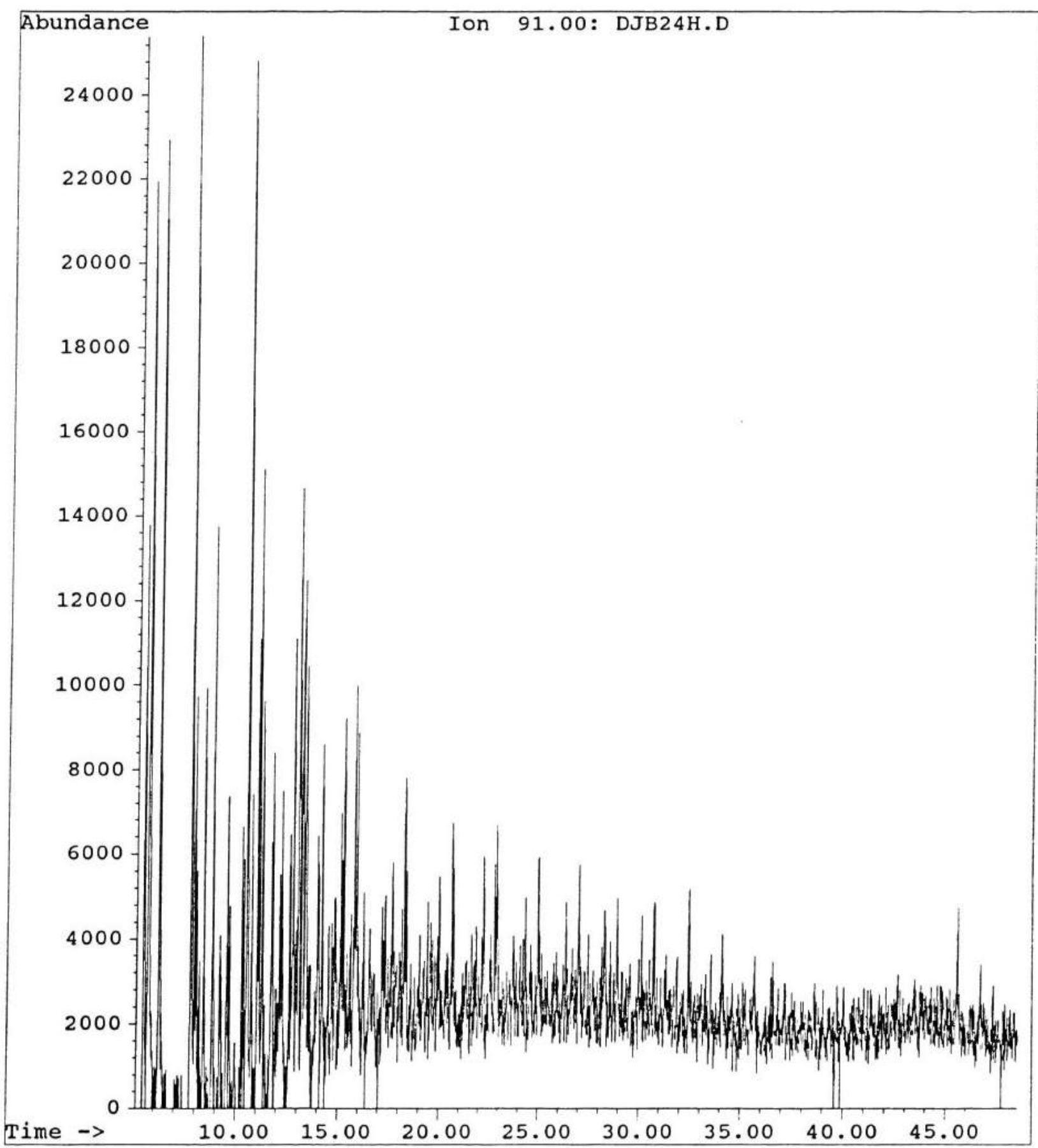


Ion $91.00:$ DJB24H.D

ARABIAN CRUDE 24 HRS WEATHERING

\begin{tabular}{|c|c|c|c|c|c|c|}
\hline Peak\# & Ret Time & Type & width & Area & Start Time & End Time \\
\hline 1 & 5.514 & $\mathrm{BV}$ & 0.059 & 534564 & 5.400 & 5.663 \\
\hline 2 & 5.719 & vV & 0.080 & 1161175 & 5.663 & 5.999 \\
\hline 3 & 6.248 & PV & 0.056 & 839682 & 6.175 & 6.372 \\
\hline 4 & 7.794 & BV & 0.051 & 859048 & 7.746 & 7.941 \\
\hline 5 & 7.976 & VV & 0.042 & 274380 & 7.941 & 8.029 \\
\hline 6 & 8.069 & VV & 0.054 & 211697 & 8.029 & 8.172 \\
\hline 7 & 8.430 & BV & 0.045 & 282127 & 8.383 & 8.519 \\
\hline 8 & 8.859 & BV & 0.046 & 398165 & 8.668 & 8.972 \\
\hline 9 & 9.576 & BV & 0.059 & 282274 & 9.504 & 9.661 \\
\hline 10 & 10.293 & BV & 0.032 & 114793 & 10.188 & 10.347 \\
\hline 11 & 10.383 & VV & 0.057 & 163844 & 10.347 & 10.468 \\
\hline 12 & 10.531 & PV & 0.064 & 1039364 & 10.468 & 10.708 \\
\hline 13 & 10.761 & VV & 0.050 & 250120 & 10.708 & 10.864 \\
\hline 14 & 11.045 & BV & 0.043 & 278730 & 10.995 & 11.076 \\
\hline 15 & 11.117 & VV & 0.047 & 408597 & 11.076 & 11.179 \\
\hline 16 & 11.284 & VV & 0.065 & 380179 & 11.225 & 11.366 \\
\hline 17 & 11.729 & BV & 0.048 & 197272 & 11.651 & 11.759 \\
\hline 18 & 11.794 & VV & 0.045 & 250663 & 11.759 & 11.865 \\
\hline 19 & 12.138 & VV & 0.051 & 176682 & 12.089 & 12.181 \\
\hline 20 & 12.224 & VV & 0.048 & 209977 & 12.181 & 12.263 \\
\hline 21 & 12.631 & VV & 0.040 & 148108 & 12.589 & 12.673 \\
\hline 22 & 12.795 & VV & 0.063 & 489569 & 12.673 & 12.900 \\
\hline 23 & 13.044 & VV & 0.042 & 359735 & 12.999 & 13.070 \\
\hline 24 & 13.086 & VV & 0.043 & 239262 & 13.070 & 13.155 \\
\hline 25 & 13.253 & VV & 0.062 & 513298 & 13.155 & 13.318 \\
\hline 26 & 13.395 & VV & 0.078 & 531280 & 13.318 & 13.488 \\
\hline 27 & 14.002 & VV & 0.095 & 372429 & 13.823 & 14.154 \\
\hline 28 & 14.215 & PV & 0.046 & 226353 & 14.154 & 14.296 \\
\hline 29 & 15.131 & BV & 0.052 & 190571 & 15.004 & 15.174 \\
\hline 30 & 15.291 & VV & 0.041 & 188377 & 15.251 & 15.337 \\
\hline 31 & 15.822 & VV & 0.047 & 259569 & 15.785 & 15.897 \\
\hline 32 & 15.943 & VV & 0.046 & 195273 & 15.897 & 15.988 \\
\hline 33 & 18.318 & PV & 0.036 & 105211 & 18.283 & 18.362 \\
\hline 34 & 20.666 & PV & 0.046 & 129047 & 20.536 & 20.705 \\
\hline 35 & 22.902 & VV & 0.061 & 202810 & 22.850 & 22.997 \\
\hline
\end{tabular}


File:

Operator:

Date Acquired:

Method File:

Sample Name:

Misc Info:

ALS vial:
C: \CHEMPC \DATA \DJB24H.D

BOYE

21 Jul 94 11:20 pm

JAFFE.M

ARABIAN CRUDE 24 HRS WEATHERING

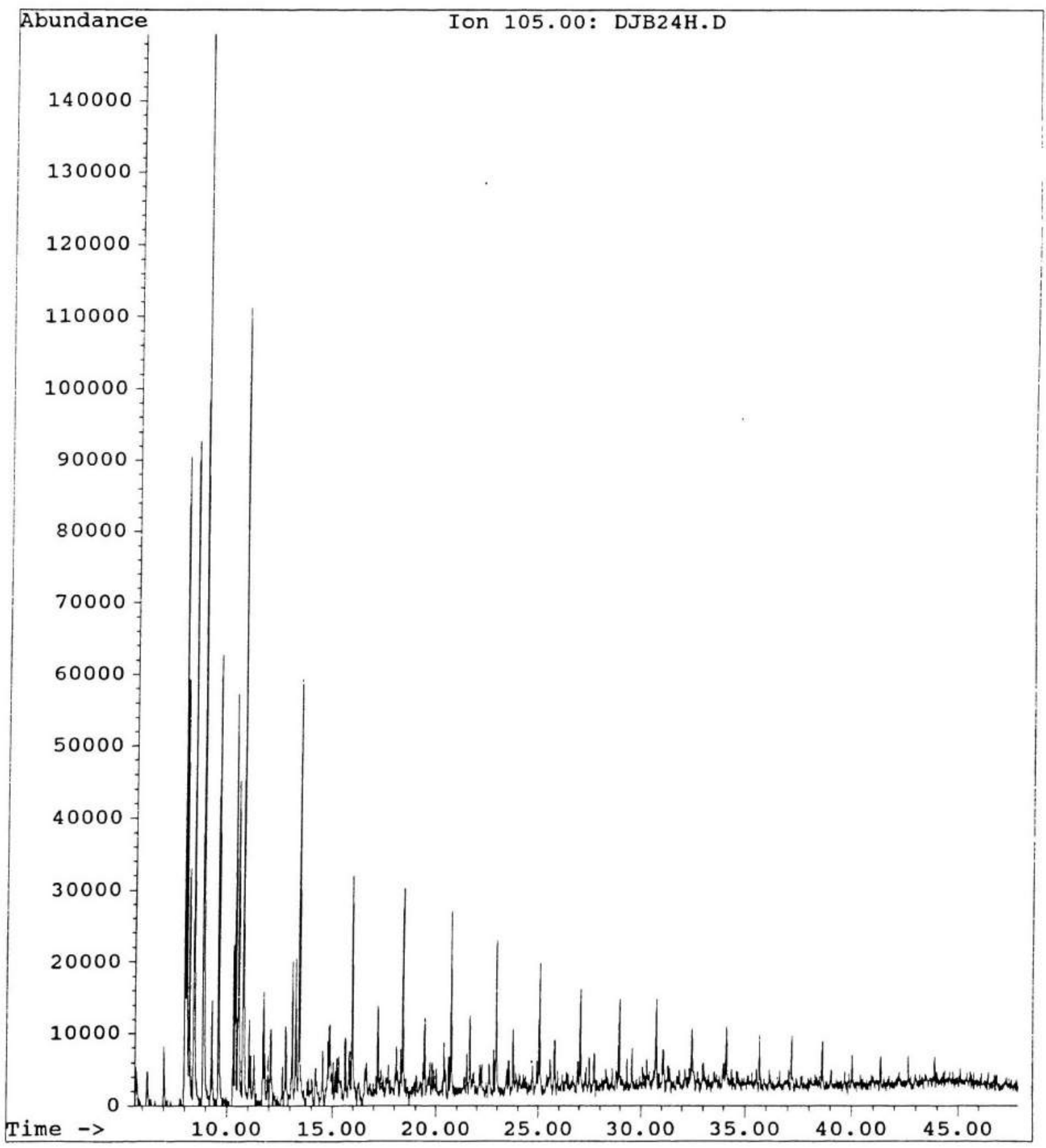


Ion $105.00:$ DJB2 $4 \mathrm{H} . \mathrm{D}$

ARABIAN CRUDE 24 HRS WEATHERING

$\begin{array}{crlrrrr}\text { Peak\# } & \text { Ret Time } & \text { Type } & \text { Width } & \text { Area } & \text { Start Time } & \text { End Time } \\ 1 & 7.981 & \text { BV } & 0.042 & 2354123 & 7.927 & 8.029 \\ 2 & 8.065 & \text { VV } & 0.050 & 1943312 & 8.029 & 8.158 \\ 3 & 8.204 & \text { VV } & 0.049 & 1114629 & 8.158 & 8.364 \\ 4 & 8.430 & \text { VV } & 0.049 & 2979188 & 8.364 & 8.734 \\ 5 & 8.859 & \text { BV } & 0.046 & 4394759 & 8.782 & 9.060 \\ 6 & 9.269 & \text { BV } & 0.048 & 439776 & 9.219 & 9.394 \\ 7 & 9.581 & \text { BV } & 0.049 & 1986160 & 9.513 & 9.753 \\ 8 & 10.291 & \text { BV } & 0.040 & 561193 & 10.235 & 10.338 \\ 9 & 10.384 & \text { VV } & 0.044 & 1566313 & 10.338 & 10.467 \\ 10 & 10.520 & \text { VV } & 0.060 & 1853958 & 10.467 & 10.701 \\ 11 & 10.762 & \text { VV } & 0.042 & 3019127 & 10.701 & 10.910 \\ 12 & 11.041 & \text { BV } & 0.035 & 195332 & 10.976 & 11.085 \\ 13 & 11.723 & \text { BV } & 0.050 & 409661 & 11.651 & 11.767 \\ 14 & 12.792 & \text { PV } & 0.067 & 393235 & 12.693 & 12.895 \\ 15 & 13.090 & \text { VV } & 0.070 & 655587 & 13.000 & 13.182 \\ 16 & 13.255 & \text { VV } & 0.050 & 672593 & 13.182 & 13.350 \\ 17 & 13.417 & \text { VV } & 0.049 & 1943444 & 13.350 & 13.598 \\ 18 & 14.858 & \text { VV } & 0.058 & 420772 & 14.808 & 14.913 \\ 19 & 15.946 & \text { VV } & 0.045 & 846711 & 15.857 & 16.043 \\ 20 & 17.192 & \text { PV } & 0.045 & 354248 & 17.138 & 17.286 \\ 21 & 18.395 & \text { VV } & 0.044 & 777419 & 18.332 & 18.494 \\ 22 & 20.736 & \text { VV } & 0.043 & 704616 & 20.676 & 20.838 \\ 23 & 21.645 & \text { PV } & 0.043 & 302376 & 21.569 & 21.778 \\ 24 & 22.947 & \text { PV } & 0.044 & 597780 & 22.874 & 23.042 \\ 25 & 25.049 & \text { BV } & 0.047 & 510763 & 24.989 & 25.182 \\ 26 & 27.050 & \text { VV } & 0.052 & 473130 & 26.983 & 27.210 \\ 27 & 28.950 & \text { VV } & 0.040 & 294796 & 28.885 & 28.991 \\ 28 & 30.755 & \text { BV } & 0.052 & 407683 & 30.660 & 30.842\end{array}$


File:

Operator:

Date Acquired: 21 Jul 9

Method File:

Sample Name:

Misc Info:

ALS vial:

BOYE

JAFFE.M
C: $\backslash$ CHEMPC $\backslash$ DATA $\backslash D J B 24 H . D$

$11: 20 \mathrm{pm}$

ARABIAN CRUDE 24 HRS WEATHERING

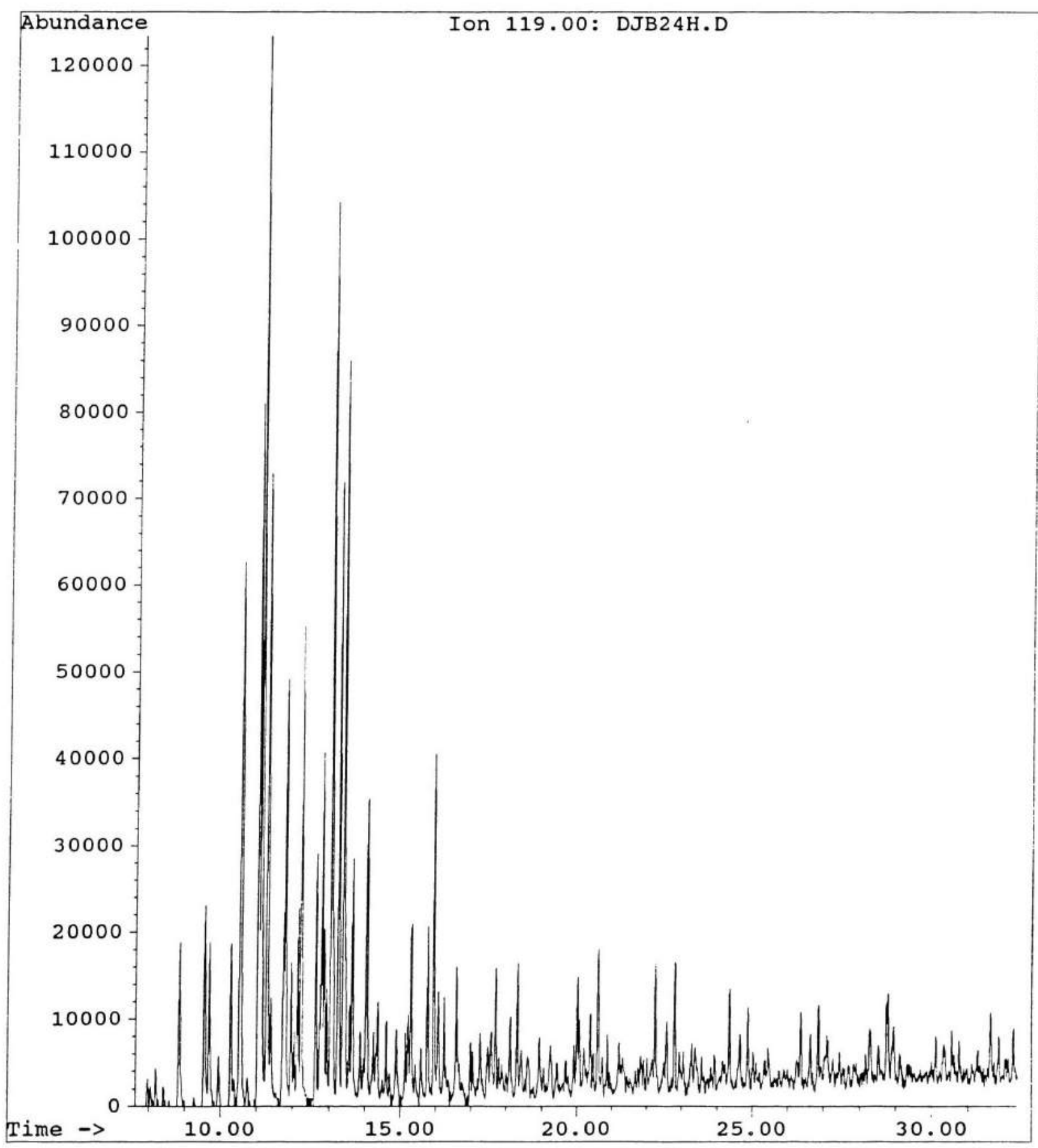


Ion $119.00:$ DJB24H.D

ARABIAN CRUDE 24 HRS WEATHERING

$\begin{array}{crlrrrr}\text { Peak\# } & \text { Ret Time } & \text { Type } & \text { Width } & \text { Area } & \text { Start Time } & \text { End Time } \\ 1 & 8.859 & \text { BV } & 0.048 & 572898 & 8.810 & 8.981 \\ 2 & 9.553 & \text { BV } & 0.057 & 794139 & 9.494 & 9.647 \\ 3 & 9.694 & \text { VB } & 0.047 & 567069 & 9.647 & 9.815 \\ 4 & 10.291 & \text { BV } & 0.044 & 537708 & 10.235 & 10.360 \\ 5 & 10.553 & \text { BV } & 0.055 & 2227031 & 10.463 & 10.695 \\ 6 & 11.042 & \text { BV } & 0.038 & 1885520 & 10.986 & 11.077 \\ 7 & 11.116 & \text { VV } & 0.043 & 3485029 & 11.077 & 11.220 \\ 8 & 11.282 & \text { VV } & 0.045 & 2173655 & 11.220 & 11.364 \\ 9 & 11.740 & \text { BV } & 0.034 & 474764 & 11.660 & 11.757 \\ 10 & 11.793 & \text { VV } & 0.048 & 1550716 & 11.757 & 11.914 \\ 11 & 11.958 & \text { VV } & 0.040 & 437133 & 11.914 & 12.012 \\ 12 & 12.135 & \text { VV } & 0.039 & 563617 & 12.086 & 12.181 \\ 13 & 12.225 & \text { VB } & 0.043 & 1460699 & 12.181 & 12.411 \\ 14 & 12.630 & \text { PV } & 0.041 & 734363 & 12.512 & 12.696 \\ 15 & 12.792 & \text { VV } & 0.046 & 1222924 & 12.696 & 12.827 \\ 16 & 12.852 & \text { VV } & 0.046 & 555314 & 12.827 & 12.899 \\ 17 & 13.040 & \text { VV } & 0.046 & 3092775 & 12.987 & 13.181 \\ 18 & 13.250 & \text { VV } & 0.043 & 2009044 & 13.181 & 13.332 \\ 19 & 13.387 & \text { VV } & 0.047 & 2658366 & 13.332 & 13.512 \\ 20 & 13.643 & \text { VB } & 0.044 & 819275 & 13.599 & 13.798 \\ 21 & 14.051 & \text { VV } & 0.047 & 1057136 & 13.991 & 14.157 \\ 22 & 15.297 & \text { VV } & 0.041 & 508360 & 15.254 & 15.381 \\ 23 & 15.764 & \text { BV } & 0.041 & 486516 & 15.717 & 15.841 \\ 24 & 15.935 & \text { PV } & 0.041 & 972834 & 15.884 & 16.015 \\ 25 & 16.581 & \text { PB } & 0.051 & 512149 & 16.440 & 16.701 \\ 26 & 18.311 & \text { PV } & 0.041 & 360205 & 18.213 & 18.366 \\ 27 & 20.607 & \text { PV } & 0.046 & 437345 & 20.538 & 20.685 \\ 28 & 22.807 & \text { VV } & 0.042 & 395072 & 22.753 & 22.856\end{array}$


File:

Operator:

Date Acquired: 21 Jul

Method File:

Sample Name:

Misc Info:

ALS vial:

BOYE

1
$C: \backslash C H E M P C \backslash D A T A \backslash D J B 24 \mathrm{H}, D$

JAFFE.M

$11: 20 \mathrm{pm}$

ARABIAN CRUDE 24 HRS WEATHERING

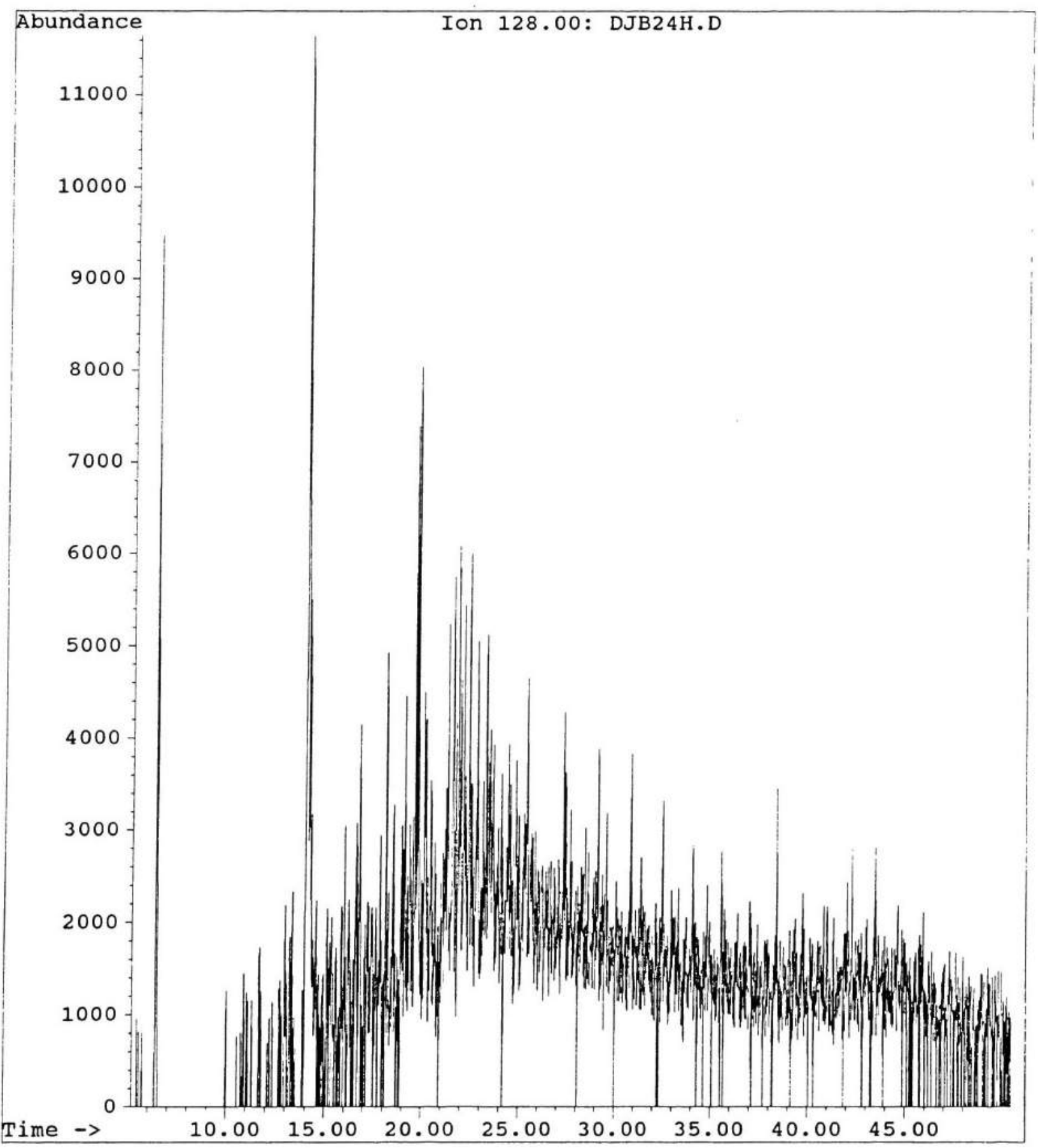


Ion $128.00:$ DUB $4 \mathrm{H} . \mathrm{D}$

ARABIAN CRUDE 24 HRS WEATHERING

$\begin{array}{crlrrrr}\text { Peak\# } & \text { Ret Time } & \text { Type } & \text { width } & \text { Area } & \text { Start Time } & \text { End Time } \\ 1 & 6.392 & \text { BB } & 0.051 & 333424 & 6.340 & 6.502 \\ 2 & 14.039 & \text { VV } & 0.075 & 62243 & 13.934 & 14.153 \\ 3 & 14.200 & \text { VV } & 0.054 & 108664 & 14.153 & 14.269 \\ 4 & 16.013 & \text { VV } & 0.045 & 79188 & 15.971 & 16.067 \\ 5 & 16.795 & \text { VB } & 0.074 & 191566 & 16.716 & 16.889 \\ 6 & 18.170 & \text { VV } & 0.044 & 144595 & 18.097 & 18.222 \\ 7 & 18.545 & \text { VV } & 0.058 & 138789 & 18.480 & 18.588 \\ 8 & 18.965 & \text { VV } & 0.058 & 118172 & 18.892 & 19.013 \\ 9 & 19.144 & \text { VV } & 0.053 & 150641 & 19.081 & 19.188 \\ 10 & 19.694 & \text { VV } & 0.068 & 281577 & 19.593 & 19.737 \\ 11 & 19.793 & \text { VV } & 0.073 & 319102 & 19.737 & 19.872 \\ 12 & 20.126 & \text { VV } & 0.048 & 125824 & 20.086 & 20.170 \\ 13 & 21.357 & \text { VV } & 0.085 & 250589 & 21.234 & 21.475 \\ 14 & 21.622 & \text { VV } & 0.089 & 290380 & 21.475 & 21.709 \\ 15 & 21.884 & \text { VV } & 0.057 & 197733 & 21.807 & 21.978 \\ 16 & 22.006 & \text { VV } & 0.049 & 109448 & 21.978 & 22.078 \\ 17 & 22.165 & \text { VV } & 0.053 & 141006 & 22.078 & 22.222 \\ 18 & 22.443 & \text { VV } & 0.050 & 138998 & 22.379 & 22.525 \\ 19 & 22.863 & \text { VV } & 0.061 & 127984 & 22.841 & 23.019 \\ 20 & 23.325 & \text { VV } & 0.048 & 120703 & 23.274 & 23.387 \\ 21 & 25.443 & \text { VV } & 0.044 & 81593 & 25.406 & 25.486\end{array}$

247 
File:

Operator:

Date Acquired:

Method File:

Sample Name:

Misc Info:

ALS vial:
C: \CHEMPC \DATA \DJB24H.D

BOYE

21 Jul 94 11:20 pm

ARABIAN CRUDE 24 HRS WEATHERING

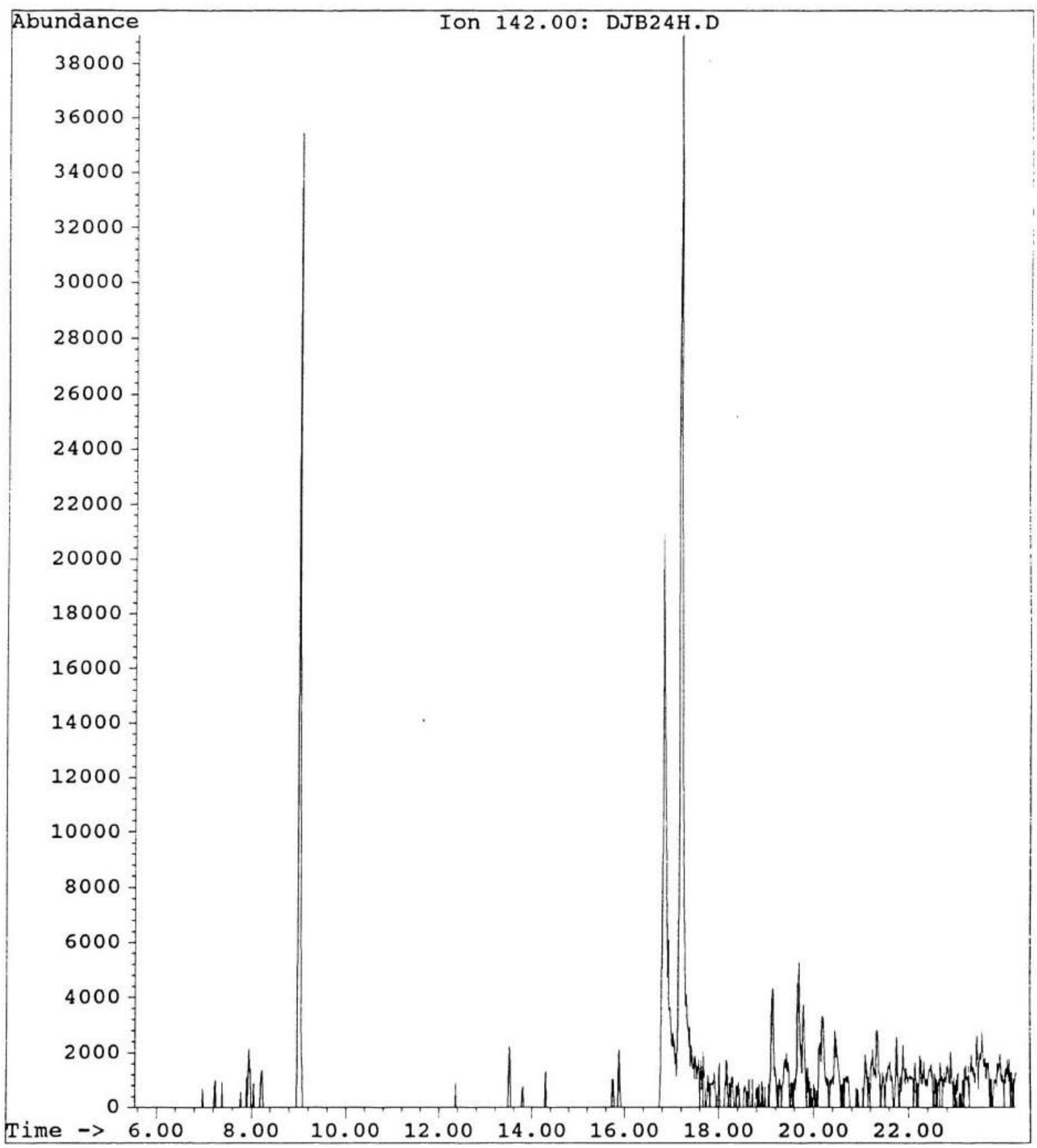


Ion $142.00:$ DJB2 $4 \mathrm{H} . \mathrm{D}$

ARABIAN CRUDE 24 HRS WEATHERING

$\begin{array}{crllrrr}\text { Peak\# } & \text { Ret Time } & \text { Type } & \text { Width } & \text { Area } & \text { Start Time } & \text { End Time } \\ 1 & 9.005 & \text { BB } & 0.042 & 906209 & 8.943 & 9.095 \\ 2 & 16.828 & \text { BV } & 0.076 & 1073078 & 16.743 & 17.019 \\ 3 & 17.176 & \text { PV } & 0.059 & 1548111 & 17.114 & 17.382 \\ 4 & 19.696 & \text { PV } & 0.058 & 147253 & 19.584 & 19.755\end{array}$


File:

Operator:

Date Acquired:

Method File:

Sample Name:

Misc Info:

ALS vial:
C: \CHEMPC \DATA \DJB24H.D

BOYE

21 Jul $94 \quad 11: 20 \mathrm{pm}$

JAFFE.M

ARABIAN CRUDE 24 HRS WEATHERING

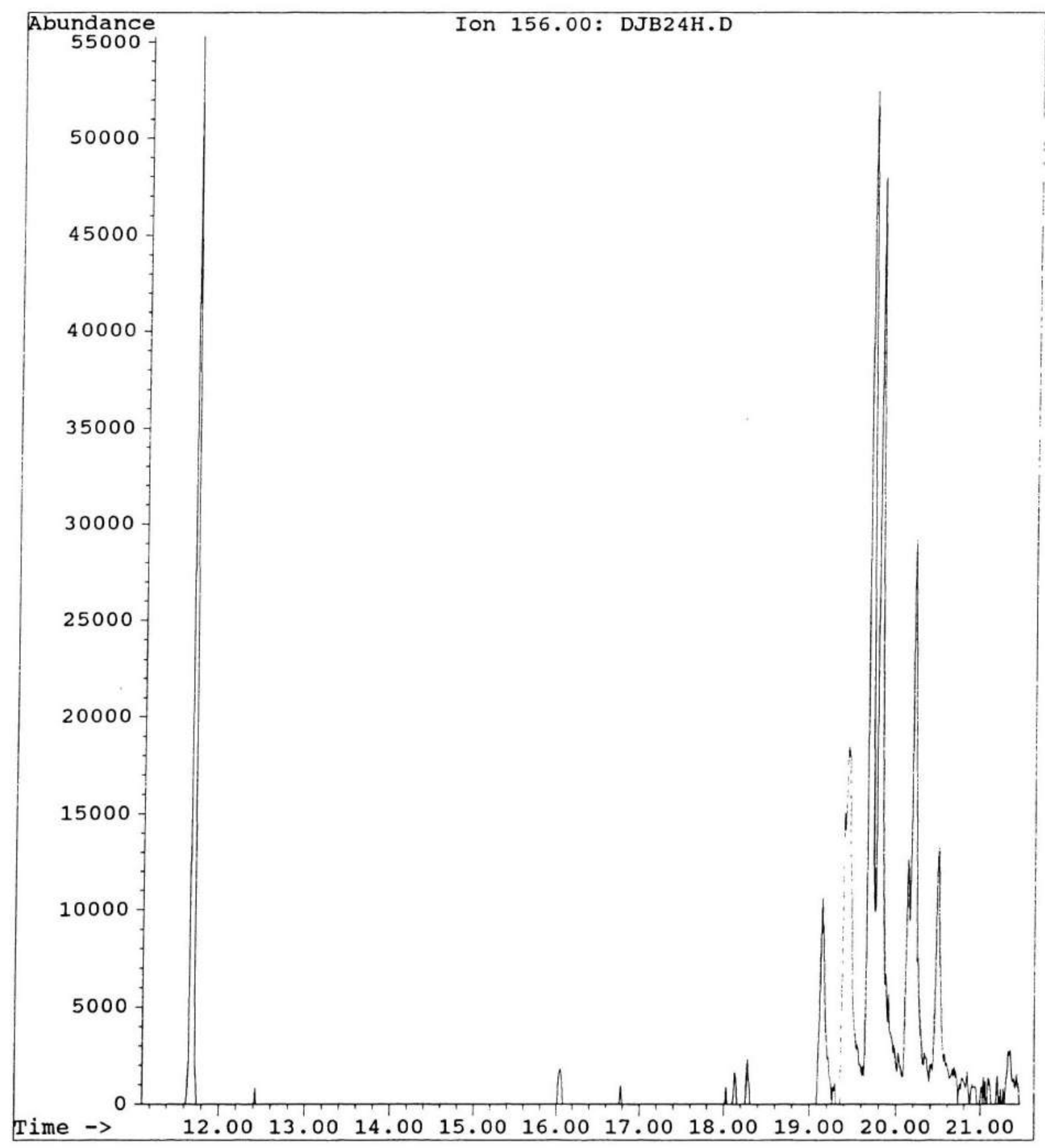


ARABIAN CRUDE 24 HRS WEATHERING

$\begin{array}{crlrrrr}\text { Peak\# } & \text { Ret Time } & \text { Type } & \text { Width } & \text { Area } & \text { Start Time } & \text { End Time } \\ 1 & 11.692 & \text { BB } & 0.038 & 1233748 & 11.613 & 11.755 \\ 2 & 19.147 & \text { BB } & 0.062 & 463159 & 19.080 & 19.317 \\ 3 & 19.439 & \text { BV } & 0.089 & 1209774 & 19.355 & 19.628 \\ 4 & 19.691 & \text { VV } & 0.055 & 1809720 & 19.628 & 19.748 \\ 5 & 19.796 & \text { VV } & 0.058 & 1988298 & 19.748 & 20.012 \\ 6 & 20.135 & \text { VV } & 0.039 & 305129 & 20.076 & 20.153 \\ 7 & 20.199 & \text { VV } & 0.060 & 1167148 & 20.153 & 20.391 \\ 8 & 20.489 & \text { VV } & 0.059 & 485242 & 20.391 & 20.636\end{array}$


File:

Operator:

Date Acquired: 21 Jul

Method File:

Sample Name:

Misc Info:

ALS vial:

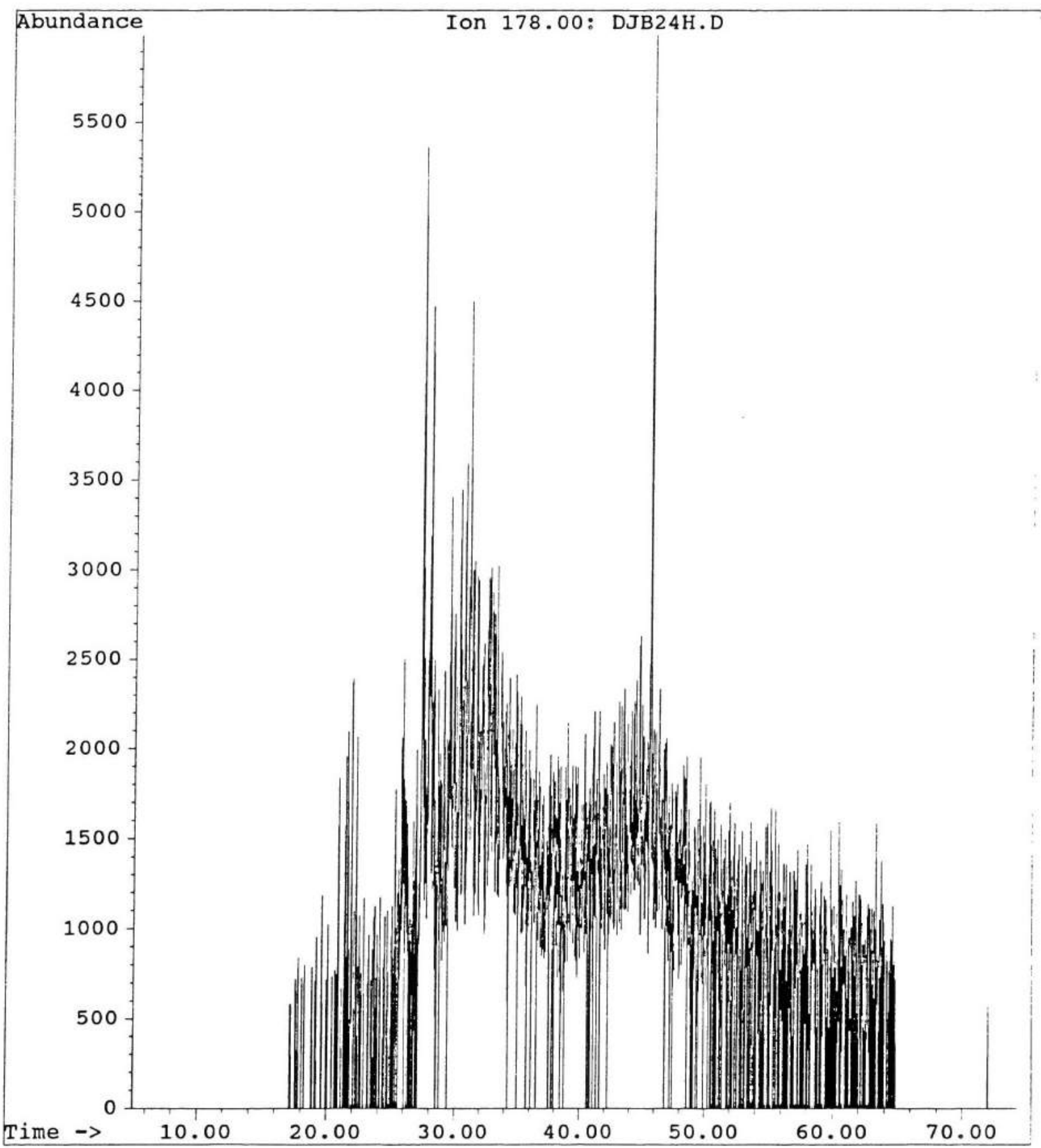


Ion $178.00:$ DJB2 $4 \mathrm{H} . \mathrm{D}$

ARABIAN CRUDE 24 HRS WEATHERING

$\begin{array}{crlrrrr}\text { Peak\# } & \text { Ret Time } & \text { Type } & \text { Width } & \text { Area } & \text { Start Time } & \text { End Time } \\ 1 & 20.868 & \text { BB } & 0.050 & 49168 & 20.771 & 20.904 \\ 2 & 21.406 & \text { VV } & 0.046 & 52304 & 21.358 & 21.433 \\ 3 & 21.577 & \text { PV } & 0.078 & 85700 & 21.433 & 21.673 \\ 4 & 21.877 & \text { BB } & 0.055 & 88125 & 21.825 & 21.949 \\ 5 & 22.262 & \text { BB } & 0.048 & 59009 & 22.148 & 22.310 \\ 6 & 25.279 & \text { BV } & 0.035 & 25356 & 25.226 & 25.312 \\ 7 & 25.752 & \text { VV } & 0.064 & 99067 & 25.701 & 25.818 \\ 8 & 25.871 & \text { VV } & 0.073 & 106298 & 25.818 & 25.929 \\ 9 & 26.933 & \text { VV } & 0.036 & 36155 & 26.897 & 26.956 \\ 10 & 27.334 & \text { VV } & 0.087 & 325499 & 27.239 & 27.417 \\ 11 & 27.436 & \text { VV } & 0.041 & 61927 & 27.417 & 27.465 \\ 12 & 27.893 & \text { VV } & 0.067 & 143744 & 27.840 & 27.938 \\ 13 & 27.974 & \text { VV } & 0.064 & 167480 & 27.938 & 28.032 \\ 14 & 28.821 & \text { VV } & 0.044 & 52684 & 28.781 & 28.848 \\ 15 & 29.094 & \text { VV } & 0.084 & 147502 & 28.958 & 29.121 \\ 16 & 29.544 & \text { VV } & 0.078 & 156491 & 29.449 & 29.571 \\ 17 & 30.310 & \text { VV } & 0.083 & 206547 & 30.262 & 30.405 \\ 18 & 30.673 & \text { VV } & 0.068 & 158582 & 30.610 & 30.714 \\ 19 & 30.733 & \text { VV } & 0.053 & 112946 & 30.714 & 30.808 \\ 20 & 30.984 & \text { VV } & 0.076 & 129074 & 30.914 & 31.010 \\ 21 & 31.064 & \text { VV } & 0.084 & 268654 & 31.010 & 31.166 \\ 22 & 32.729 & \text { VV } & 0.069 & 126143 & 32.673 & 32.761 \\ 23 & 34.316 & \text { VV } & 0.069 & 89047 & 34.249 & 34.337 \\ 24 & 37.595 & \text { VV } & 0.066 & 67182 & 37.511 & 37.629 \\ 25 & 37.829 & \text { PV } & 0.065 & 65019 & 37.767 & 37.854 \\ 26 & 41.100 & \text { PV } & 0.019 & 14882 & 41.077 & 41.122 \\ 27 & 41.665 & \text { PV } & 0.045 & 49467 & 41.637 & 41.715 \\ 28 & 45.584 & \text { VV } & 0.084 & 359945 & 45.498 & 45.717 \\ 29 & 46.866 & \text { VV } & 0.043 & 49335 & 46.821 & 46.886 \\ 30 & 50.344 & \text { PV } & 0.055 & 22411 & 50.281 & 50.361\end{array}$


File:

Operator:

Date Acquired:

Method File:

Sample Name:

Misc Info:

ALS vial:
C: \CHEMPC \DATA \DJB2 4H.D

BOYE

21 Jul 94 11:20 pm

JAFFE.M

ARABIAN CRUDE 24 HRS WEATHERING

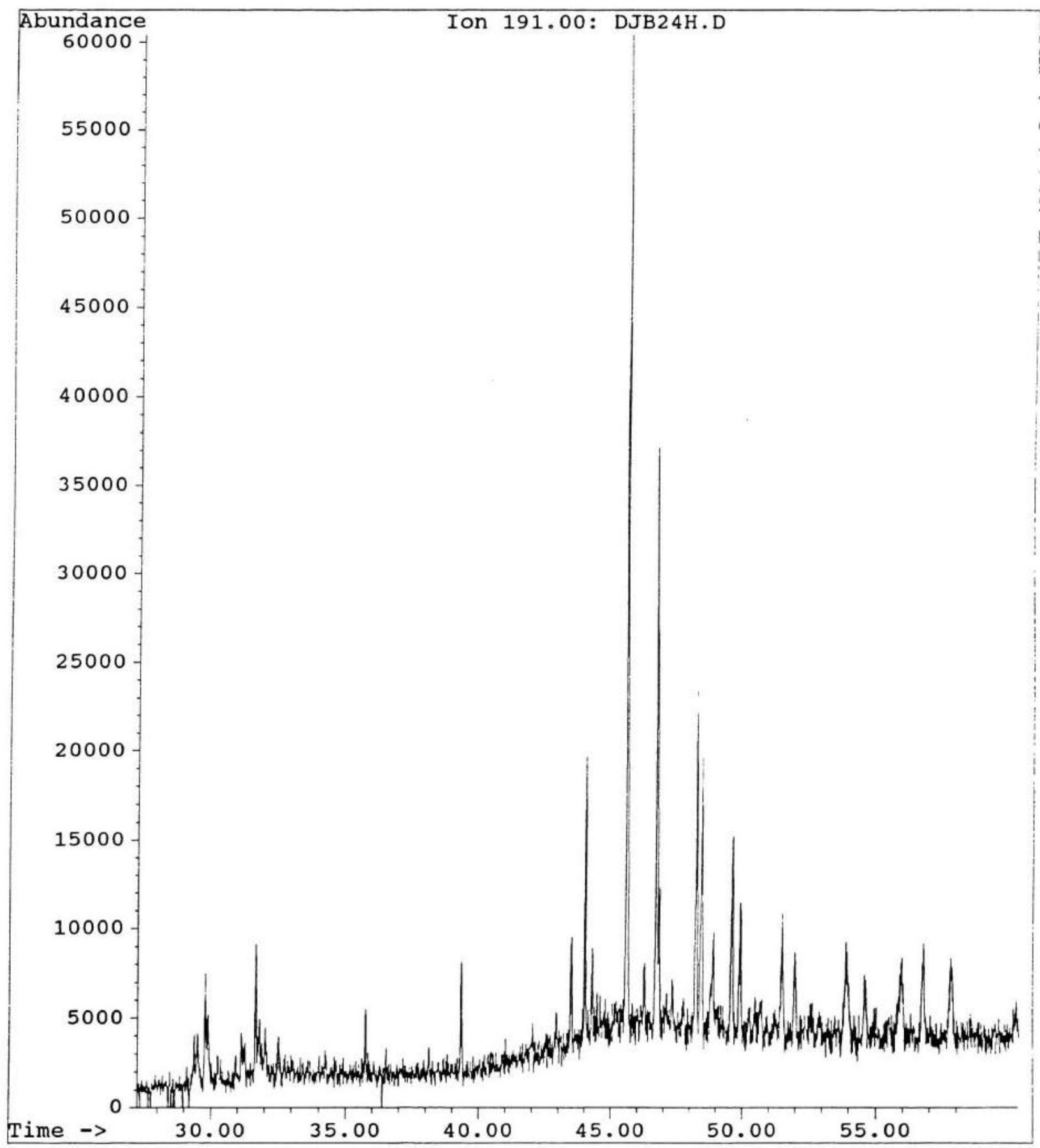


Ion $191.00:$ DJB24H.D

ARABIAN CRUDE 24 HRS WEATHERING

$\begin{array}{crlrrrr}\text { Peak\# } & \text { Ret Time } & \text { Type } & \text { Width } & \text { Area } & \text { Start Time } & \text { End Time } \\ 1 & 31.667 & \text { PV } & 0.048 & 226364 & 31.584 & 31.726 \\ 2 & 44.022 & \text { VV } & 0.073 & 477146 & 43.895 & 44.113 \\ 3 & 45.592 & \text { PV } & 0.055 & 1945395 & 45.501 & 45.711 \\ 4 & 46.734 & \text { PV } & 0.058 & 1165504 & 46.621 & 46.803 \\ 5 & 48.239 & \text { BV } & 0.070 & 863691 & 48.159 & 48.356 \\ 6 & 48.443 & \text { VV } & 0.068 & 779706 & 48.356 & 48.557 \\ 7 & 49.613 & \text { PV } & 0.080 & 554394 & 49.514 & 49.720 \\ 8 & 49.906 & \text { VV } & 0.091 & 475333 & 49.820 & 50.107\end{array}$


File:

Operator:

Date Acquired: BOYE

Method File:

Sample Name:

Misc Info:

ALS vial:

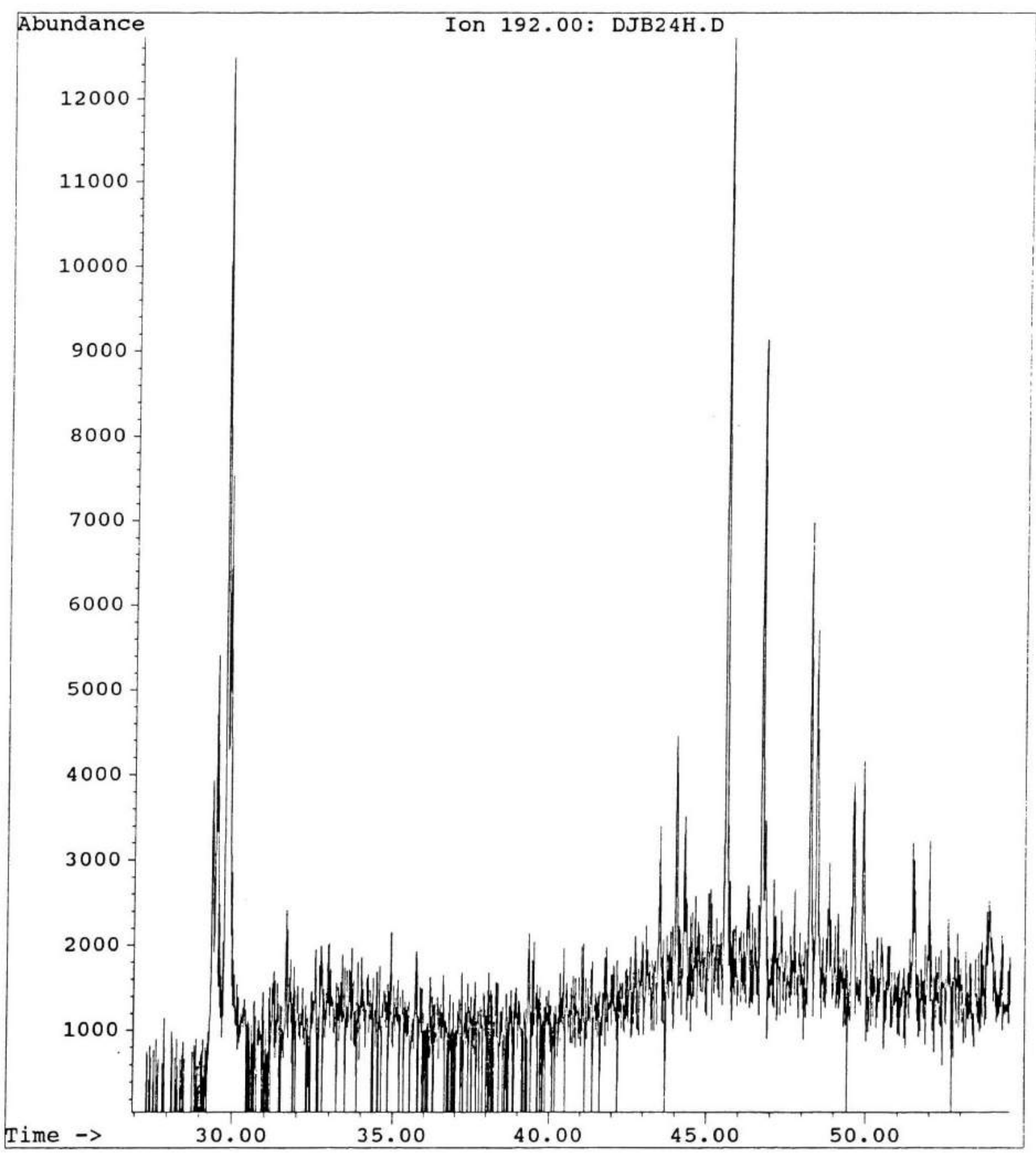


Ion $192.00:$ DJB24H.D

ARABIAN CRUDE 24 HRS WEATHERING

$\begin{array}{crlrrrr}\text { Peak\# } & \text { Ret Time } & \text { Type } & \text { Width } & \text { Area } & \text { Start Time } & \text { End Time } \\ 1 & 29.359 & \text { VV } & 0.063 & 154634 & 29.280 & 29.419 \\ 2 & 29.488 & \text { VV } & 0.077 & 233116 & 29.419 & 29.559 \\ 3 & 29.763 & \text { VV } & 0.059 & 474799 & 29.680 & 29.829 \\ 4 & 29.874 & \text { VV } & 0.085 & 335665 & 29.829 & 30.116 \\ 5 & 44.020 & \text { PV } & 0.058 & 136876 & 43.953 & 44.088 \\ 6 & 45.594 & \text { PV } & 0.067 & 475118 & 45.497 & 45.697 \\ 7 & 46.731 & \text { PV } & 0.057 & 247644 & 46.664 & 46.792 \\ 8 & 48.242 & \text { VV } & 0.063 & 224262 & 48.176 & 48.351 \\ 9 & 48.431 & \text { PV } & 0.069 & 196369 & 48.351 & 48.562 \\ 10 & 49.619 & \text { PV } & 0.076 & 154522 & 49.497 & 49.715 \\ 11 & 49.909 & \text { VV } & 0.060 & 122927 & 49.838 & 50.094\end{array}$


File:

Operator:

Date Acquired:

Method File:

Sample Name:

Misc Info:

ALS vial:
C: \CHEMPC \DATA \DJB24H.D

BOYE

21 Jul 94 11:20 pm

JAFFE.M

ARABIAN CRUDE 24 HRS WEATHERING

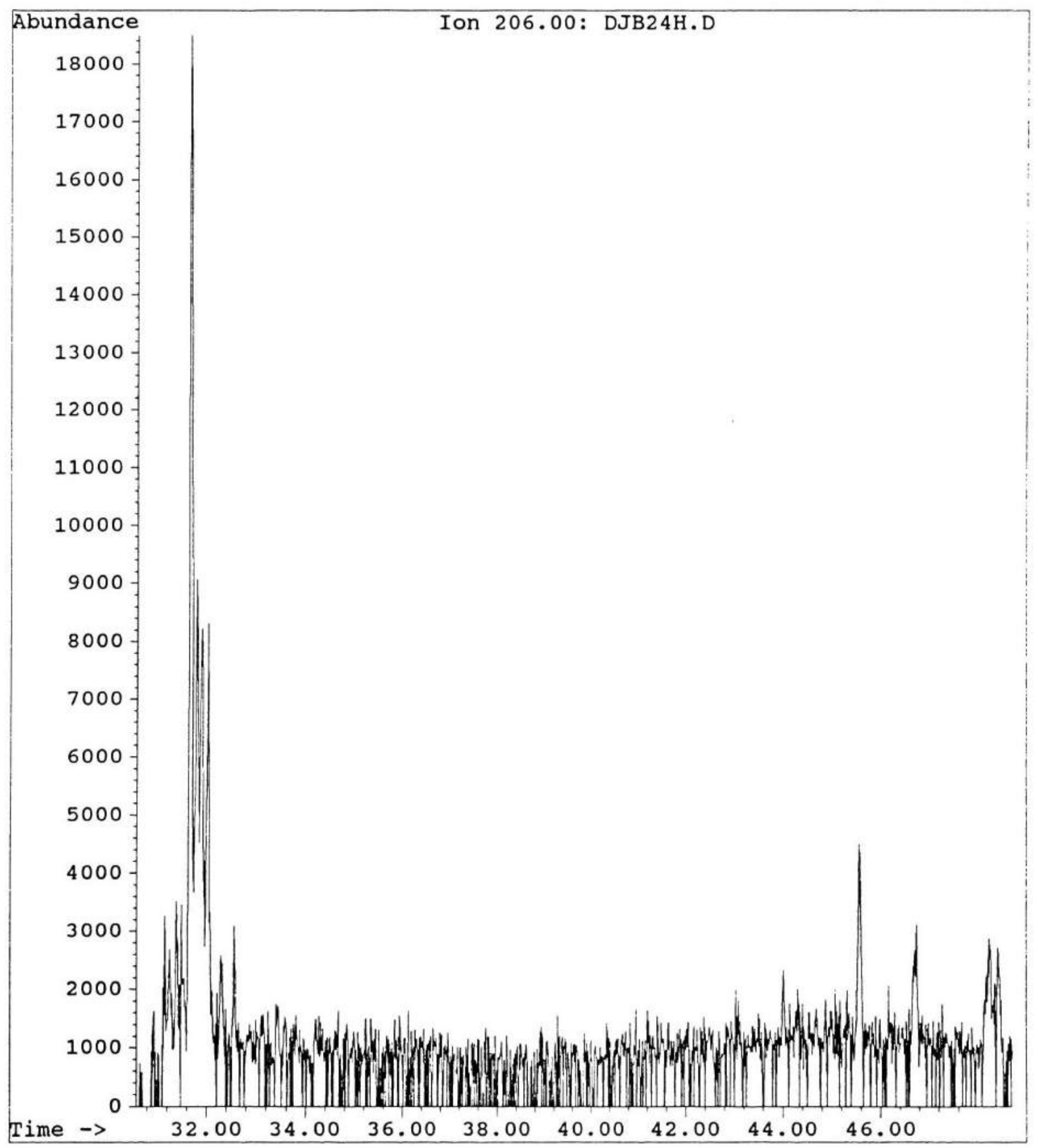


Ion $206.00:$ DJB2 4H.D

ARABIAN CRUDE 24 HRS WEATHERING

$\begin{array}{crlrrrr}\text { Peak\# } & \text { Ret Time } & \text { Type } & \text { Width } & \text { Area } & \text { Start Time } & \text { End Time } \\ 1 & 31.665 & \text { VV } & 0.064 & 749906 & 31.597 & 31.734 \\ 2 & 31.793 & \text { VV } & 0.064 & 387742 & 31.734 & 31.847 \\ 3 & 31.894 & \text { VV } & 0.077 & 377583 & 31.847 & 31.969 \\ 4 & 32.023 & \text { VV } & 0.073 & 312183 & 31.969 & 32.171\end{array}$


File:

operator:

Date Acquired:

Method File:

Sample Name:

Misc Info:

ALS vial:
C: \CHEMPC \DATA \DJB96H.D

BOYE

$23 \mathrm{Jul} 94 \quad 1: 32 \mathrm{pm}$

JAFFE.M

ARABIAN CRUDE 96 HRS WEATHERING

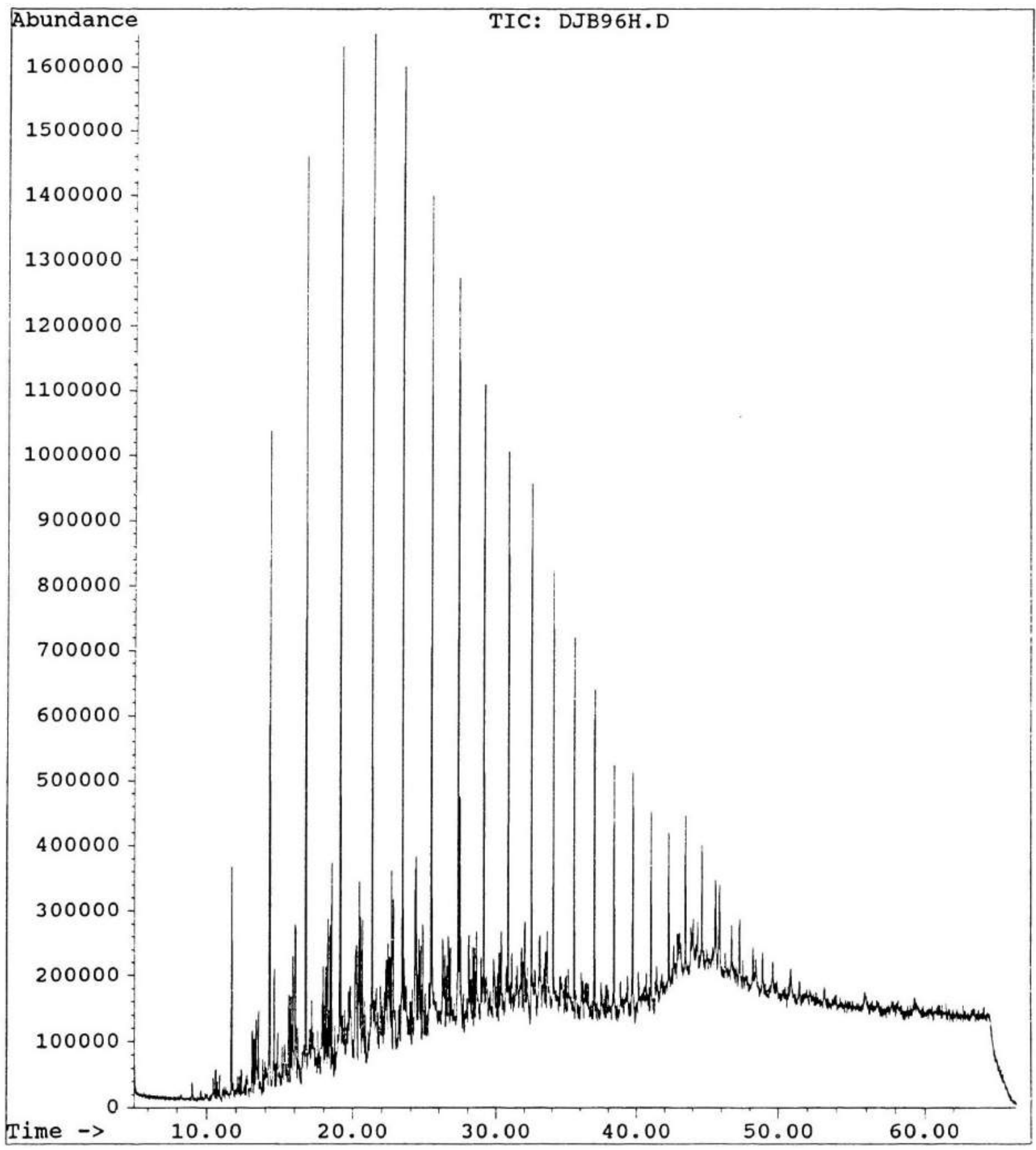


File:

Operator:

Date Acquired: $23 \mathrm{Jul} 94$

Method File: JAFFE.M

Sample Name:

Misc Info:

ALS vial:

1

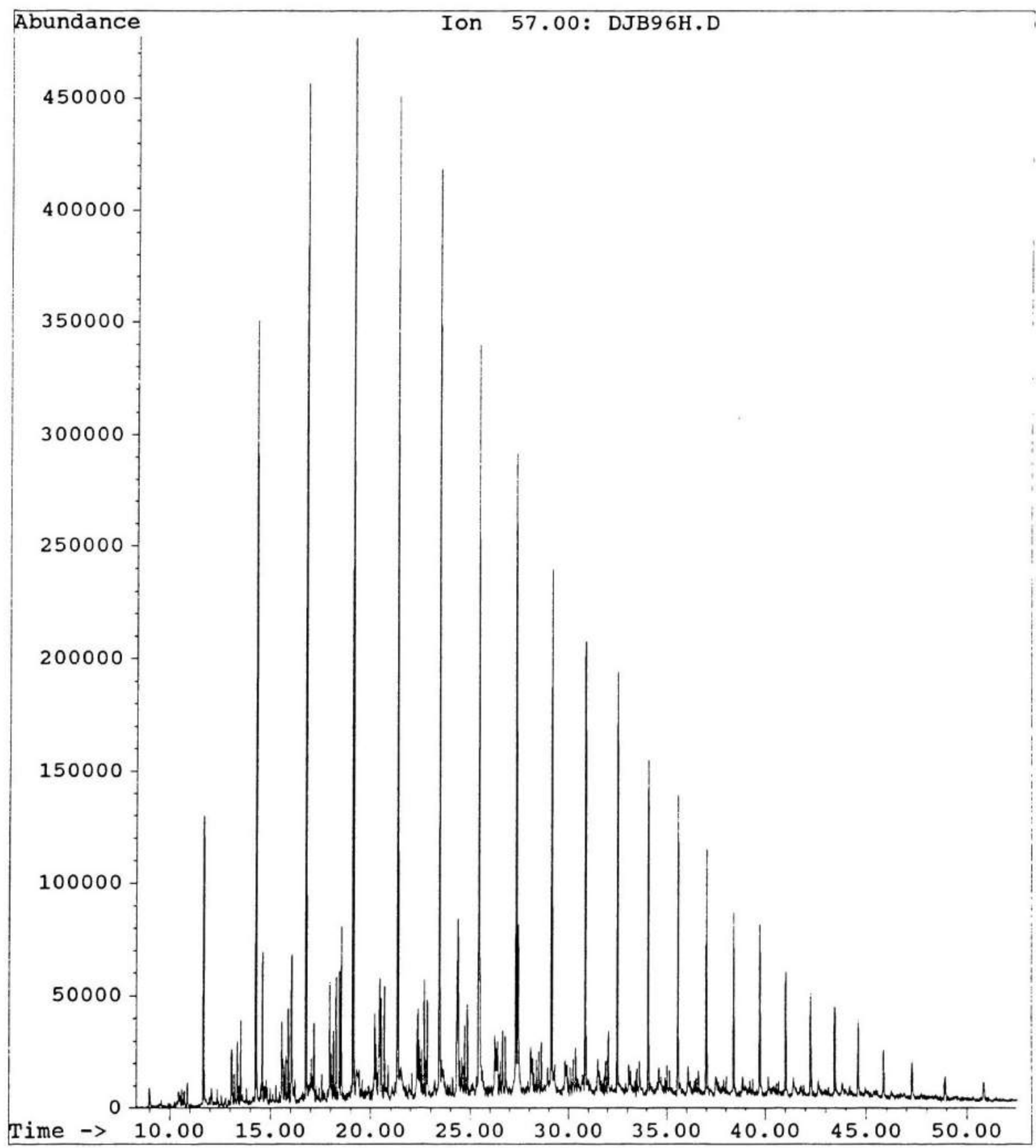


Ion $57.00:$ DJB96H.D

ARABIAN CRUDE 96 HRS WEATHERING

$\begin{array}{crlrr}\text { Peak\# } & \text { Ret Time } & \text { Type } & \text { Width } & \text { Area } \\ 1 & 11.681 & \text { BV } & 0.037 & 2928331 \\ 2 & 13.077 & \text { BV } & 0.052 & 876736 \\ 3 & 13.359 & \text { VV } & 0.040 & 713410 \\ 4 & 13.521 & \text { PV } & 0.034 & 754924 \\ 5 & 14.291 & \text { BV } & 0.036 & 7495738 \\ 6 & 14.604 & \text { VV } & 0.036 & 1465976 \\ 7 & 15.574 & \text { VV } & 0.044 & 869179 \\ 8 & 15.886 & \text { VV } & 0.042 & 1097419 \\ 9 & 16.058 & \text { PV } & 0.058 & 2201435 \\ 10 & 16.775 & \text { VV } & 0.037 & 10362197 \\ 11 & 17.159 & \text { VV } & 0.044 & 961557 \\ 12 & 17.945 & \text { VV } & 0.036 & 1151058 \\ 13 & 18.141 & \text { VV } & 0.036 & 692089 \\ 14 & 18.277 & \text { VV } & 0.037 & 1226373 \\ 15 & 18.433 & \text { PV } & 0.037 & 1301992 \\ 16 & 18.540 & \text { VB } & 0.036 & 1710197 \\ 17 & 19.125 & \text { VV } & 0.039 & 11206931 \\ 18 & 20.206 & \text { VV } & 0.048 & 1210972 \\ 19 & 20.408 & \text { VV } & 0.033 & 524641 \\ 20 & 20.456 & \text { VV } & 0.039 & 1318309 \\ 21 & 20.544 & \text { VV } & 0.039 & 1112041 \\ 22 & 20.692 & \text { PV } & 0.035 & 1102527 \\ 23 & 21.346 & \text { PV } & 0.038 & 10268157 \\ 24 & 22.347 & \text { VV } & 0.054 & 1530493 \\ 25 & 22.425 & \text { VV } & 0.058 & 992219 \\ 26 & 22.681 & \text { VB } & 0.039 & 1260449 \\ 27 & 22.831 & \text { BV } & 0.039 & 1036811 \\ 28 & 23.448 & \text { PV } & 0.038 & 9295194 \\ 29 & 24.374 & \text { PV } & 0.065 & 3360263 \\ 30 & 24.716 & \text { VV } & 0.044 & 852557 \\ 31 & 24.858 & \text { VV } & 0.039 & 962740 \\ 32 & 25.440 & \text { PV } & 0.044 & 9627379 \\ 33 & 26.250 & \text { PV } & 0.059 & 1052491 \\ 34 & 26.642 & \text { VV } & 0.040 & 715447 \\ 35 & 26.782 & \text { PV } & 0.040 & 589464 \\ 36 & 27.332 & \text { BV } & 0.041 & 7270250 \\ 37 & 27.435 & \text { VV } & 0.064 & 3027849 \\ 38 & 29.133 & \text { PV } & 0.038 & 5549979 \\ 39 & 30.852 & \text { PV } & 0.039 & 4780069 \\ 40 & 32.027 & \text { PV } & 0.043 & 725376 \\ 41 & 32.493 & \text { PV } & 0.038 & 4147903 \\ 42 & 34.064 & \text { PV } & 0.040 & 3667123 \\ 43 & 35.568 & \text { PV } & 0.037 & 2934020 \\ 44 & 37.014 & \text { PV } & 0.039 & 2481931 \\ 45 & 38.399 & \text { VV } & 0.043 & 2320386 \\ 46 & 39.733 & \text { BV } & 0.041 & 1969888 \\ 47 & 41.023 & \text { VB } & 0.045 & 1541760 \\ 48 & 42.262 & \text { BV } & 0.046 & 1335463 \\ 49 & 43.458 & \text { PV } & 0.040 & 1003595 \\ 50 & 44.620 & \text { PV } & 0.046 & 951773\end{array}$

$\begin{array}{rr}\text { Start Time } & \text { End Time } \\ 11.501 & 11.774 \\ 13.029 & 13.176 \\ 13.277 & 13.463 \\ 13.463 & 13.571 \\ 14.208 & 14.444 \\ 14.553 & 14.663 \\ 15.519 & 15.604 \\ 15.794 & 15.968 \\ 15.968 & 16.115 \\ 16.702 & 16.875 \\ 17.110 & 17.237 \\ 17.886 & 17.982 \\ 18.090 & 18.216 \\ 18.216 & 18.335 \\ 18.335 & 18.486 \\ 18.486 & 18.602 \\ 19.058 & 19.235 \\ 20.138 & 20.240 \\ 20.355 & 20.422 \\ 20.422 & 20.501 \\ 20.501 & 20.639 \\ 20.639 & 20.756 \\ 21.270 & 21.452 \\ 22.235 & 22.385 \\ 22.385 & 22.514 \\ 22.629 & 22.740 \\ 22.774 & 22.893 \\ 23.283 & 23.526 \\ 24.206 & 24.429 \\ 24.665 & 24.774 \\ 24.774 & 24.908 \\ 25.359 & 25.587 \\ 26.121 & 26.290 \\ 26.581 & 26.696 \\ 26.696 & 26.836 \\ 27.103 & 27.383 \\ 27.383 & 27.614 \\ 29.022 & 29.195 \\ 30.790 & 30.946 \\ 31.971 & 32.097 \\ 32.430 & 32.594 \\ 33.831 & 34.124 \\ 35.500 & 35.624 \\ 36.824 & 37.068 \\ 38.075 & 38.530 \\ 39.640 & 39.868 \\ 40.950 & 41.178 \\ 42.027 & 42.379 \\ 43.365 & 43.537 \\ 44.499 & 44.705\end{array}$


File:

Operator:

Date Acquired:

Method File:

Sample Name:

Misc Info:

ALS vial:
C: \CHEMPC \DATA \DJB96H.D

BOYE

23 Jul 94 1:32 pm

ARABIAN CRUDE 96 HRS WEATHERING

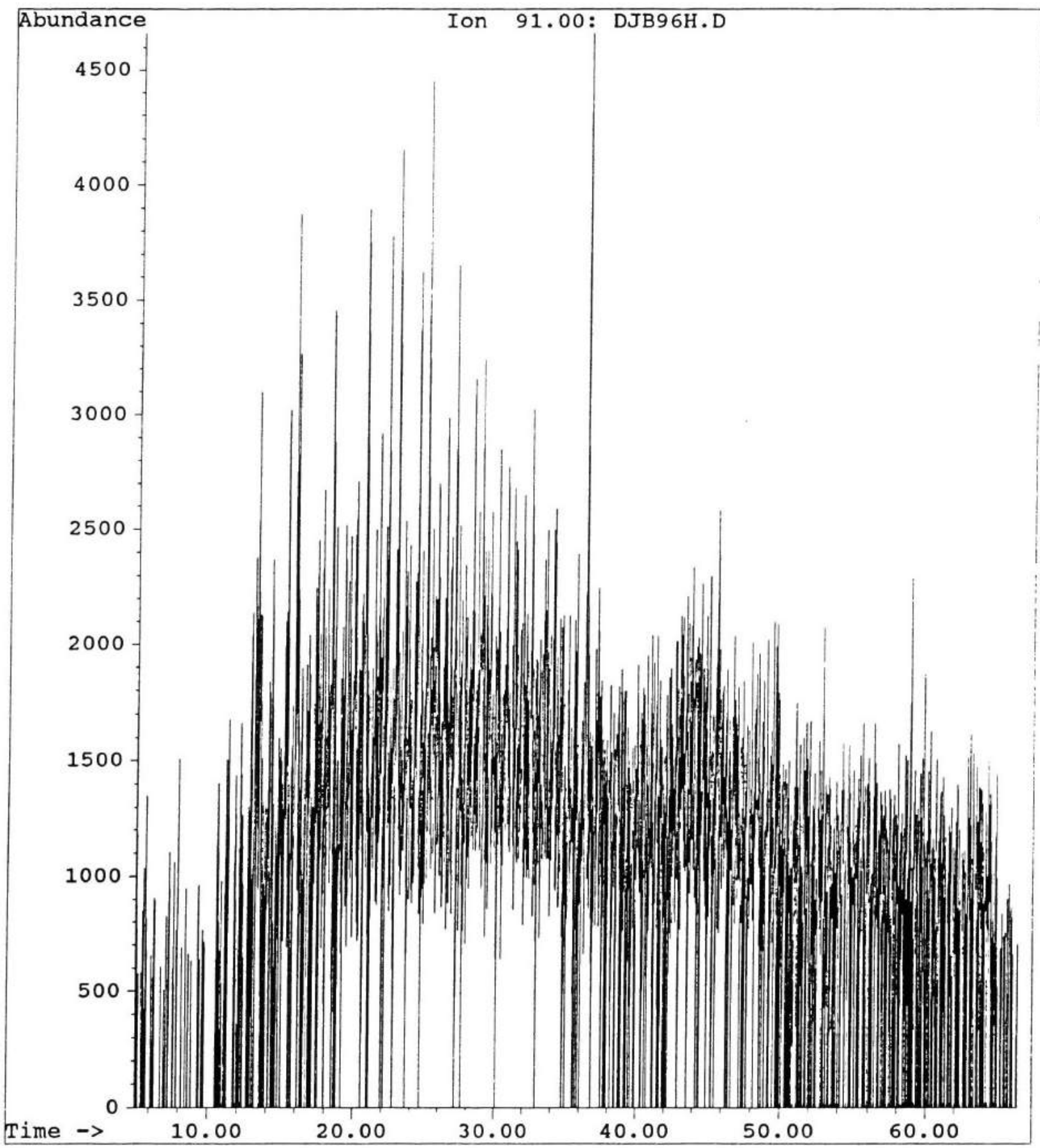


File:

Operator:

Date Acquired: 23 Jul 9

Method File: JAFFE.M

Sample Name:

Misc Info:

ALS vial:

BOYE
C: \CHEMPC \DATA \DJB96H.D

$1: 32 \mathrm{pm}$

ARABIAN CRUDE 96 HRS WEATHERING

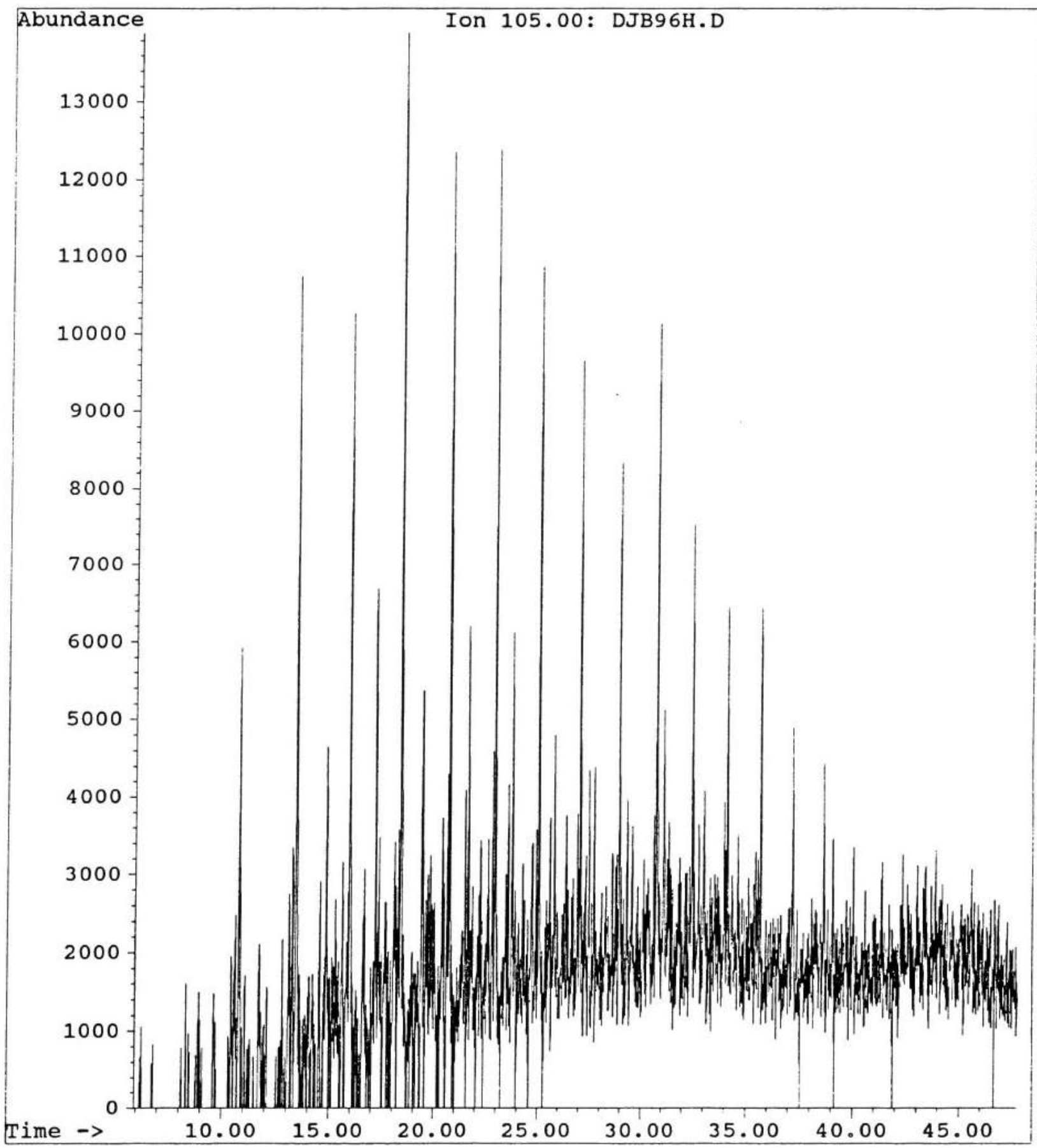


Ion 105.00: DJB96H.D
ARABIAN CRUDE 96 HRS WEATHERING

$\begin{array}{crlrrrr}\text { Peak\# } & \text { Ret Time } & \text { Type } & \text { Width } & \text { Area } & \text { Start Time } & \text { End Time } \\ 1 & 10.798 & \text { VV } & 0.057 & 209457 & 10.742 & 10.879 \\ 2 & 13.443 & \text { PV } & 0.054 & 393736 & 13.343 & 13.560 \\ 3 & 14.852 & \text { VV } & 0.063 & 219936 & 14.820 & 14.990 \\ 4 & 15.957 & \text { VV } & 0.057 & 439591 & 15.867 & 16.057 \\ 5 & 17.198 & \text { PV } & 0.038 & 127060 & 17.143 & 17.239 \\ 6 & 18.403 & \text { VV } & 0.049 & 444463 & 18.342 & 18.483 \\ 7 & 19.405 & \text { PV } & 0.039 & 86929 & 19.323 & 19.421 \\ 8 & 19.447 & \text { VV } & 0.046 & 133208 & 19.421 & 19.514 \\ 9 & 20.633 & \text { PV } & 0.045 & 111494 & 20.584 & 20.681 \\ 10 & 20.741 & \text { VV } & 0.049 & 381240 & 20.681 & 20.850 \\ 11 & 21.652 & \text { PV } & 0.045 & 162037 & 21.570 & 21.714 \\ 12 & 22.953 & \text { VV } & 0.042 & 300307 & 22.884 & 23.045 \\ 13 & 23.780 & \text { BV } & 0.054 & 138365 & 23.698 & 23.852 \\ 14 & 25.056 & \text { PV } & 0.049 & 316850 & 24.970 & 25.172 \\ 15 & 27.051 & \text { VV } & 0.048 & 266719 & 26.997 & 27.125 \\ 16 & 28.948 & \text { PV } & 0.049 & 230750 & 28.874 & 29.010 \\ 17 & 30.762 & \text { VV } & 0.053 & 335005 & 30.659 & 30.868 \\ 18 & 32.479 & \text { VV } & 0.051 & 198317 & 32.366 & 32.526 \\ 19 & 34.127 & \text { PV } & 0.043 & 136129 & 34.070 & 34.180 \\ 20 & 35.696 & \text { VV } & 0.037 & 114998 & 35.651 & 35.745\end{array}$


File:

C: \CHEMPC \DATA \DJB96H.D

Operator:

BOYE

Date Acquired: 23 Jul 94
Method File:

Sample Name:

$1: 32 \mathrm{pm}$

Misc Info:

ALS vial:

ARABIAN CRUDE 96 HRS WEATHERING

1

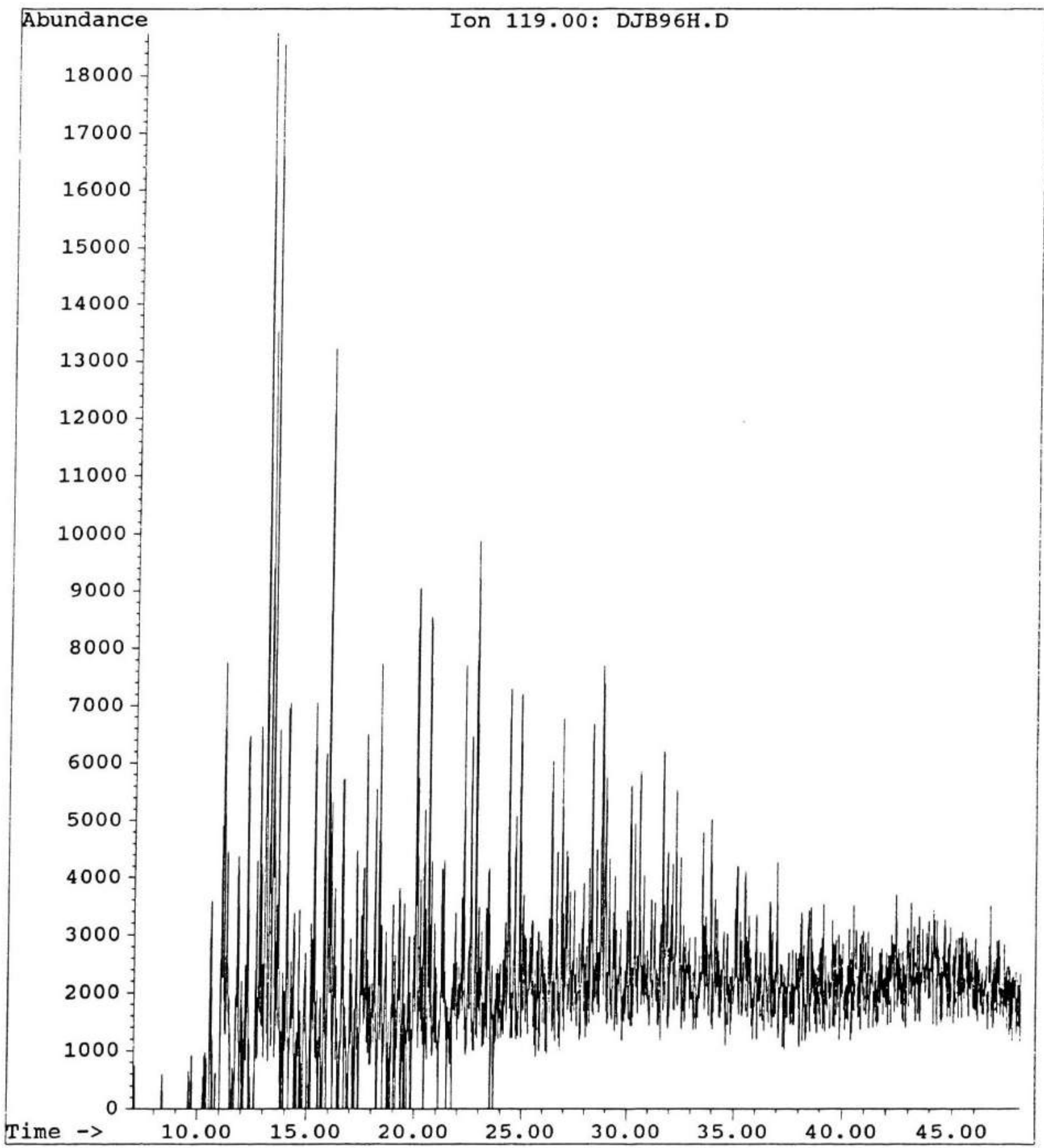


Ion 119.00: DJB96H.D

ARABIAN CRUDE 96 HRS WEATHERING

\begin{tabular}{|c|c|c|c|c|c|c|}
\hline Peak\# & Ret Time & Type & width & Area & Start Time & End Time \\
\hline 1 & 11.074 & BV & 0.044 & 130448 & 10.982 & 11.105 \\
\hline 2 & 11.144 & VV & 0.064 & 337528 & 11.105 & 11.265 \\
\hline 3 & 11.314 & VV & 0.068 & 215990 & 11.265 & 11.396 \\
\hline 4 & 11.814 & VV & 0.067 & 193620 & 11.780 & 11.920 \\
\hline 5 & 12.248 & VV & 0.059 & 278272 & 12.198 & 12.404 \\
\hline 6 & 12.658 & BV & 0.049 & 109420 & 12.557 & 12.717 \\
\hline 7 & 12.806 & VV & 0.043 & 177355 & 12.770 & 12.841 \\
\hline 8 & 13.062 & VV & 0.060 & 646783 & 13.008 & 13.160 \\
\hline 9 & 13.262 & VV & 0.048 & 446895 & 13.214 & 13.350 \\
\hline 10 & 13.404 & VV & 0.052 & 637199 & 13.350 & 13.529 \\
\hline 11 & 13.655 & VV & 0.060 & 286462 & 13.617 & 13.834 \\
\hline 12 & 14.062 & VV & 0.070 & 323070 & 13.998 & 14.176 \\
\hline 13 & 15.307 & VV & 0.052 & 232887 & 15.269 & 15.396 \\
\hline 14 & 15.778 & BV & 0.059 & 246725 & 15.689 & 15.902 \\
\hline 15 & 15.948 & VV & 0.050 & 428864 & 15.902 & 16.034 \\
\hline 16 & 16.075 & VV & 0.060 & 222098 & 16.034 & 16.185 \\
\hline 17 & 16.245 & PB & 0.059 & 130754 & 16.185 & 16.329 \\
\hline 18 & 16.589 & VV & 0.060 & 200343 & 16.512 & 16.631 \\
\hline 19 & 17.263 & VV & 0.058 & 174389 & 17.225 & 17.379 \\
\hline 20 & 17.572 & VV & 0.055 & 151197 & 17.537 & 17.634 \\
\hline 21 & 17.701 & VV & 0.056 & 229462 & 17.634 & 17.758 \\
\hline 22 & 18.115 & VV & 0.053 & 195560 & 18.066 & 18.231 \\
\hline 23 & 18.320 & VV & 0.051 & 242092 & 18.231 & 18.390 \\
\hline 24 & 20.023 & VV & 0.045 & 258578 & 19.954 & 20.055 \\
\hline 25 & 20.082 & VV & 0.046 & 134878 & 20.055 & 20.147 \\
\hline 26 & 20.399 & VV & 0.046 & 125982 & 20.357 & 20.437 \\
\hline 27 & 20.613 & PV & 0.049 & 219991 & 20.563 & 20.698 \\
\hline 28 & 22.249 & VV & 0.046 & 200054 & 22.204 & 22.371 \\
\hline 29 & 22.577 & VV & 0.047 & 170872 & 22.528 & 22.649 \\
\hline 30 & 22.806 & PV & 0.054 & 250370 & 22.751 & 22.906 \\
\hline 31 & 24.365 & PV & 0.045 & 149902 & 24.301 & 24.421 \\
\hline 32 & 24.886 & BV & 0.043 & 151411 & 24.773 & 24.974 \\
\hline 33 & 26.379 & VV & 0.049 & 149871 & 26.319 & 26.464 \\
\hline 34 & 26.869 & VV & 0.042 & 137176 & 26.826 & 26.923 \\
\hline 35 & 28.295 & VV & 0.052 & 188005 & 28.226 & 28.400 \\
\hline 36 & 28.790 & VV & 0.078 & 285540 & 28.714 & 28.856 \\
\hline 37 & 30.124 & VV & 0.052 & 121362 & 30.063 & 30.206 \\
\hline 38 & 30.557 & VV & 0.041 & 112434 & 30.490 & 30.604 \\
\hline 39 & 31.646 & VV & 0.045 & 131744 & 31.592 & 31.710 \\
\hline 40 & 32.272 & VV & 0.039 & 97972 & 32.233 & 32.387 \\
\hline
\end{tabular}


File:

operator:

Date Acquired:

Method File:

Sample Name:

Misc Info:

ALS vial:
C: \CHEMPC \DATA \DJB96H.D

BOYE

23 Jul 94 1:32 pm

JAFFE.M

ARABIAN CRUDE 96 HRS WEATHERING

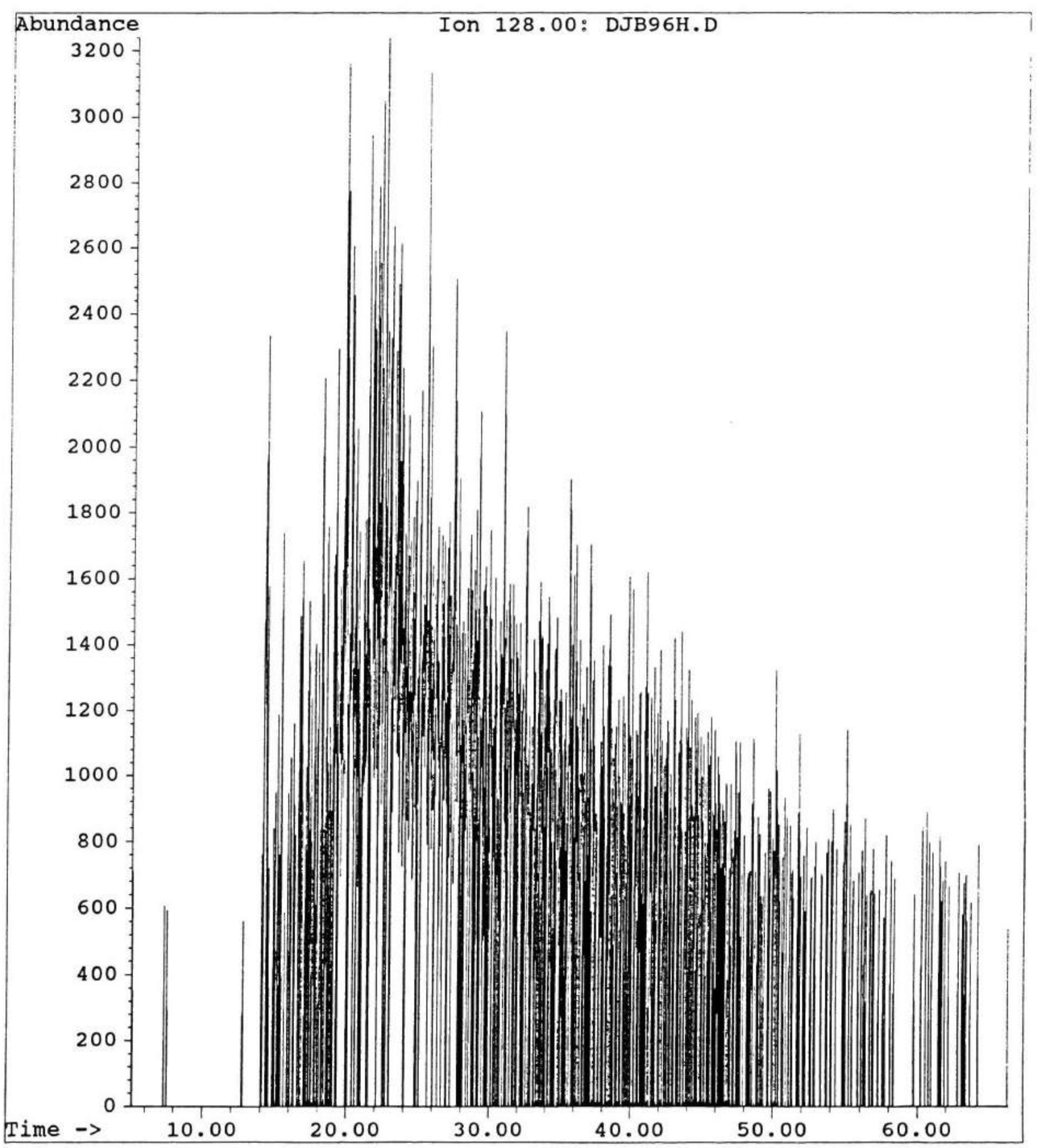


File:

Operator:

Date Acquired: $23 \mathrm{Ju}$

Method File: JAFFE.M

Sample Name:

Misc Info:

ALS vial:

BOYE

1
C: \CHEMPC \DATA \DJB96H.D

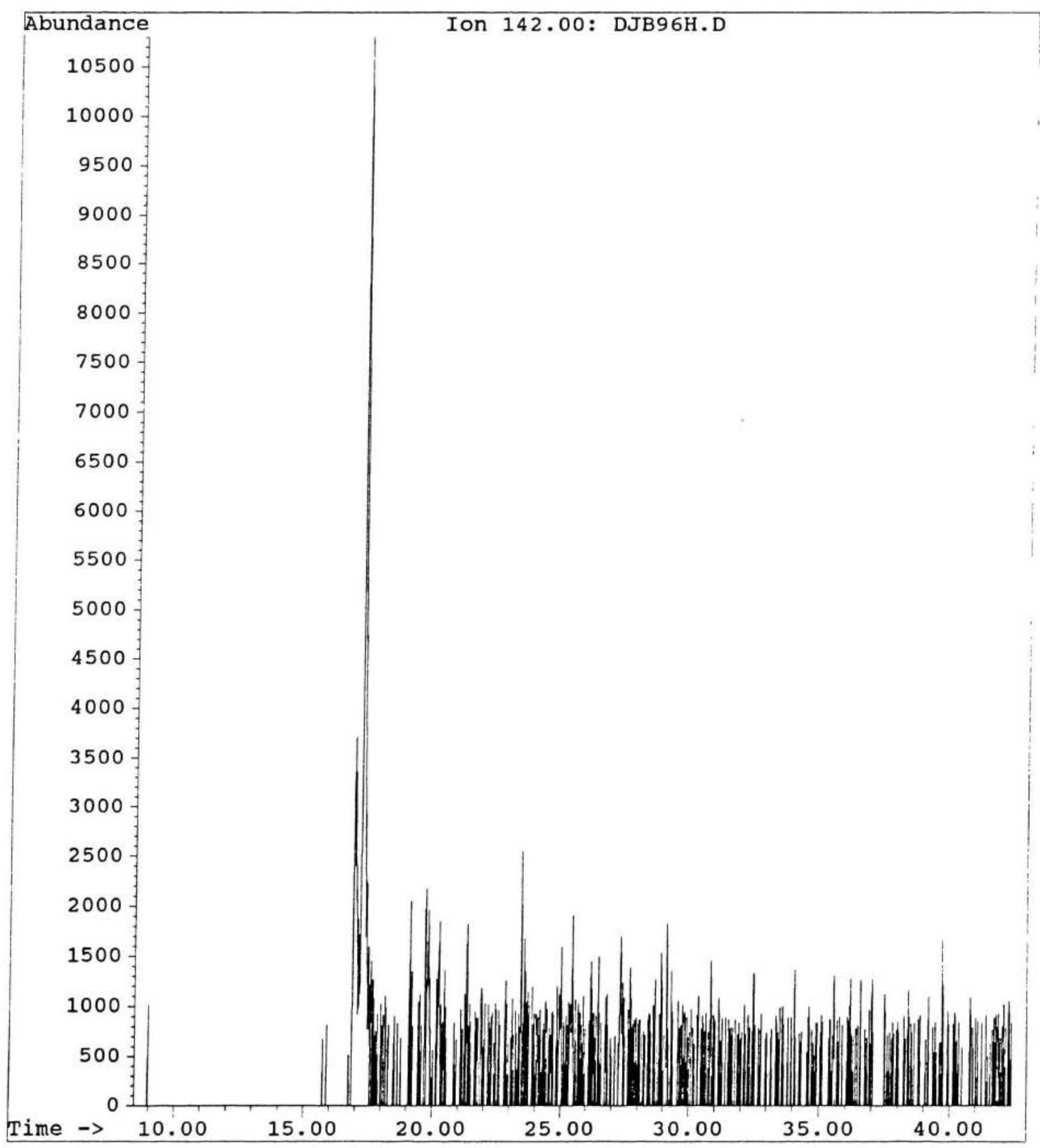


Ion $142.00:$ DJB96H.D

ARABIAN CRUDE 96 HRS WEATHERING

$\begin{array}{crlrrrr}\text { Peak\# } & \text { Ret Time } & \text { Type } & \text { Width } & \text { Area } & \text { Start Time } & \text { End Time } \\ 1 & 16.933 & \text { BV } & 0.117 & 321261 & 16.802 & 17.098 \\ 2 & 17.220 & \text { VV } & 0.077 & 633972 & 17.168 & 17.379 \\ 3 & 19.130 & \text { BV } & 0.034 & 41423 & 19.019 & 19.153 \\ 4 & 19.725 & \text { BV } & 0.047 & 69106 & 19.670 & 19.769 \\ 5 & 23.450 & \text { PV } & 0.028 & 38440 & 23.415 & 23.481\end{array}$


File:

Operator:

Date Acquired: 23 Jul 94

Method File: JAFFE.M

Sample Name:

Misc Info:

ALS vial:

BOYE
C: \CHEMPC \DATA \DJB96H.D

$1: 32 \mathrm{pm}$

ARABIAN CRUDE 96 HRS WEATHERING

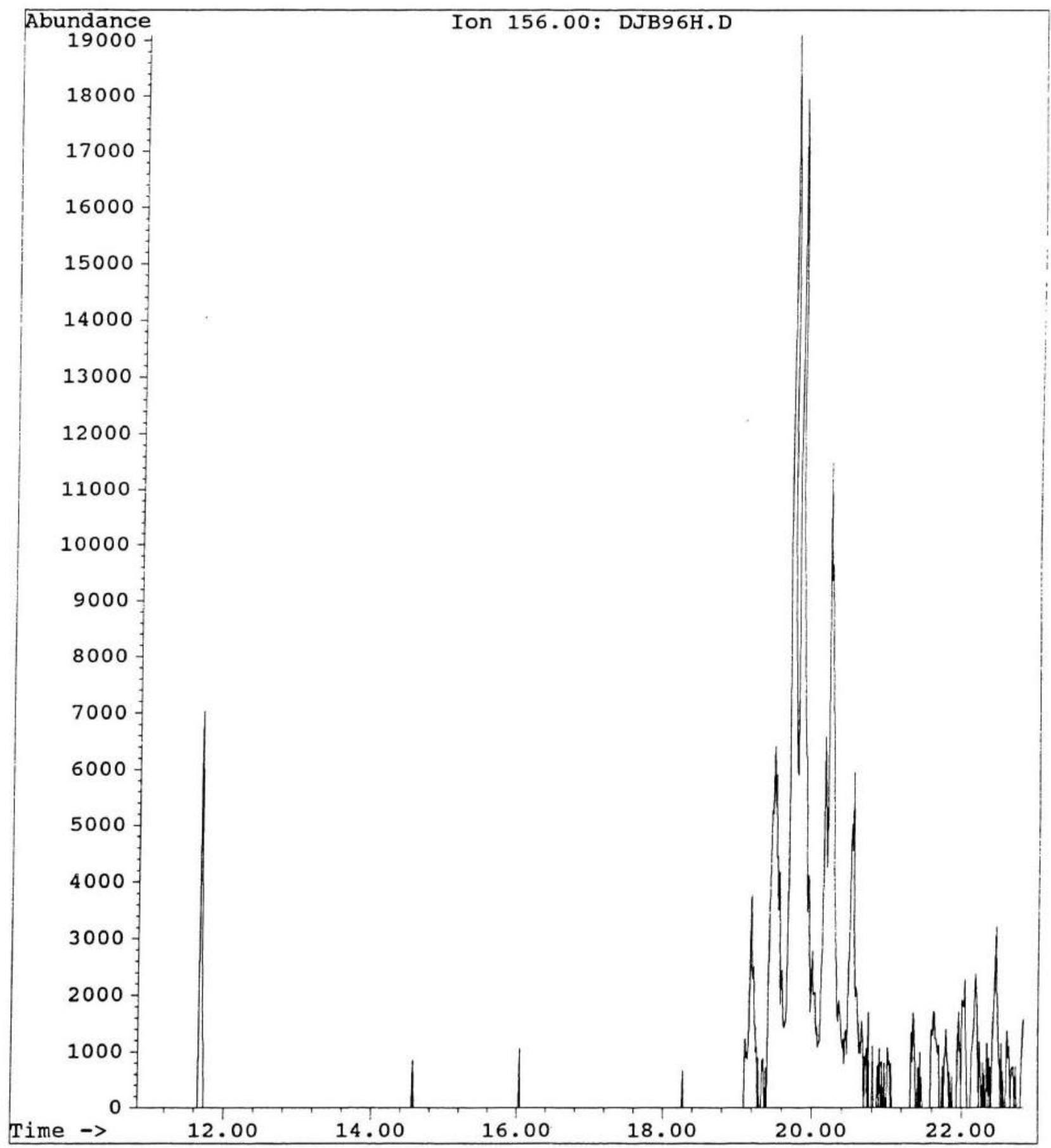


ARABIAN CRUDE 96 HRS WEATHERING

$\begin{array}{crllr}\text { Peak\# } & \text { Ret Time } & \text { Type } & \text { Width } & \text { Area } \\ 1 & 11.686 & \text { BB } & 0.041 & 147081 \\ 2 & 19.482 & \text { PV } & 0.086 & 407573 \\ 3 & 19.723 & \text { VV } & 0.062 & 718847 \\ 4 & 19.829 & \text { VV } & 0.065 & 755588 \\ 5 & 20.155 & \text { BV } & 0.041 & 146804 \\ 6 & 20.226 & \text { VV } & 0.065 & 474427 \\ 7 & 20.537 & \text { VV } & 0.075 & 194343\end{array}$

Start Time

11.642

19.379

19.637

19.786

20.094

20.185

20.468
End Time

11.746

19.579

19.786

19.985

20.185

20.435

20.648 
File:

Operator:

Date Acquired: 23 Jul 94

Method File: JAFFE.M

Sample Name:

Misc Info:

ALS vial:
C: $\backslash$ CHEMPC $\backslash$ DATA $\backslash$ DJB96H.D

BOYE

1

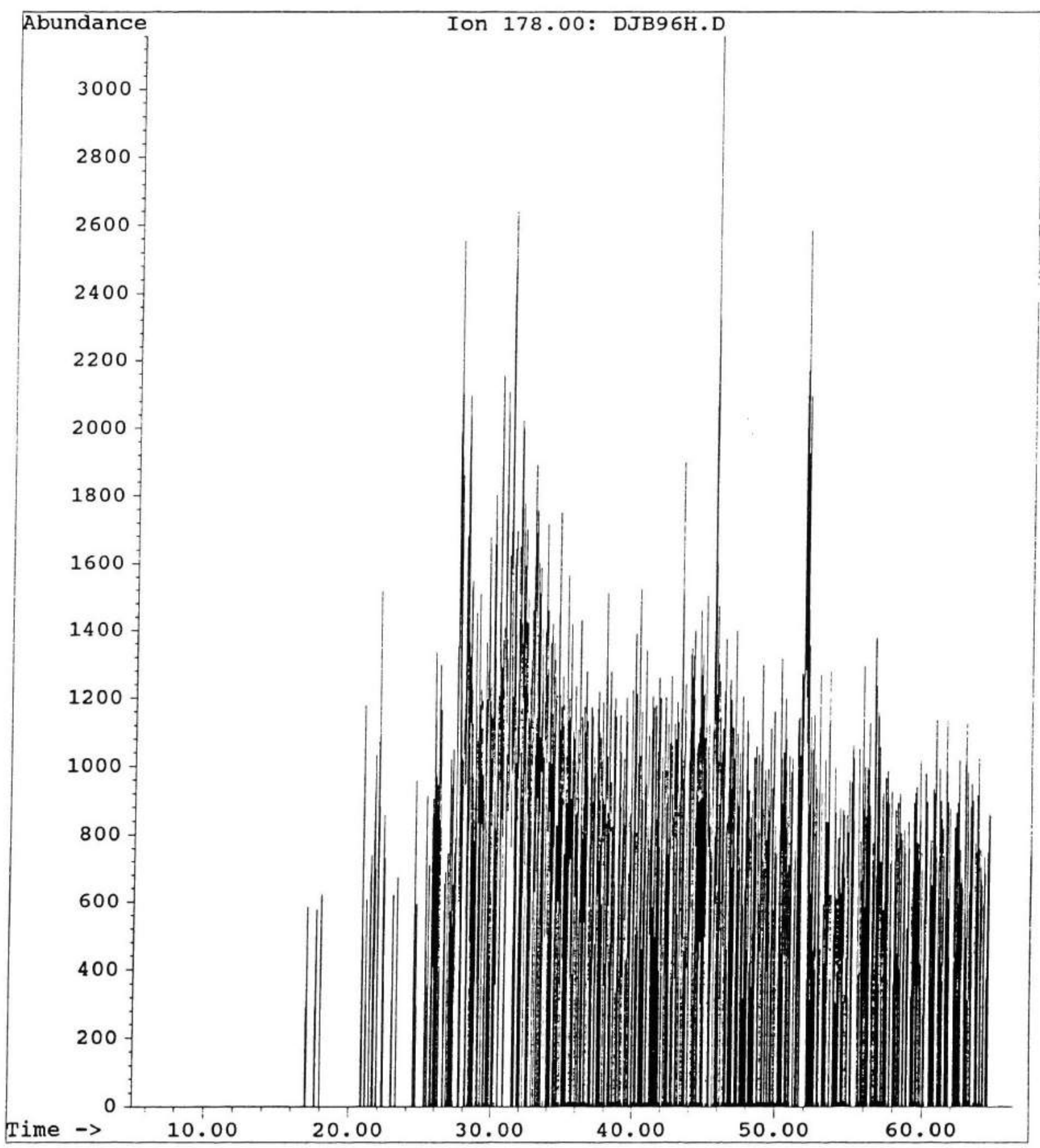


File:

operator:

C: \CHEMPC \DATA \DJB96H.D

Date Acquired: $23 \mathrm{Jul} 94$

Method File:

JAFFE.M

Sample Name:

$1: 32 \mathrm{pm}$

Misc Info:

ALS vial:

ARABIAN CRUDE 96 HRS WEATHERING

1

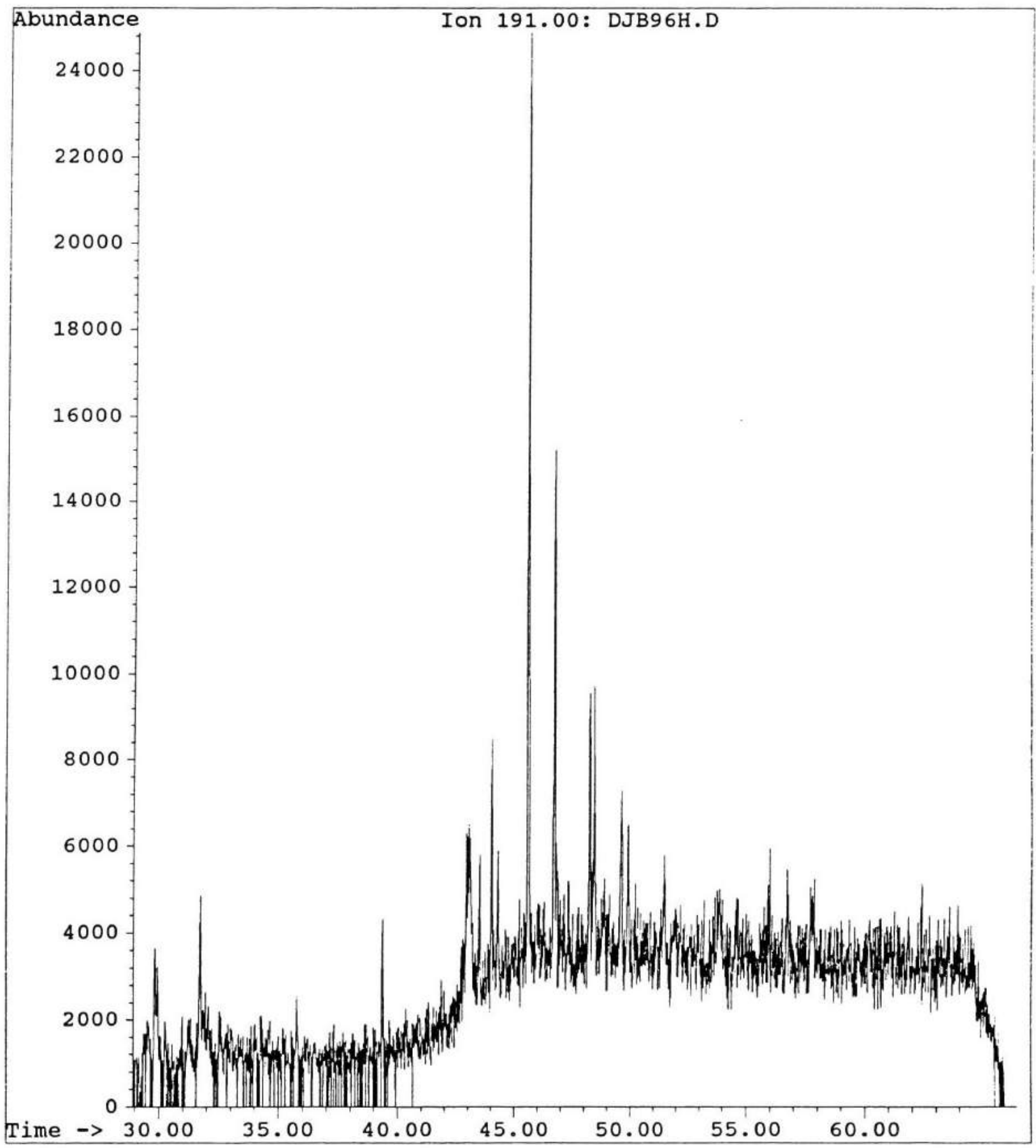


Ion 191.00: DJB96H.D

ARABIAN CRUDE 96 HRS WEATHERING

$\begin{array}{crlrrrr}\text { Peak\# } & \text { Ret Time } & \text { Type } & \text { width } & \text { Area } & \text { Start Time } & \text { End Time } \\ 1 & 31.708 & \text { PV } & 0.062 & 155021 & 31.550 & 31.786 \\ 2 & 42.925 & \text { VV } & 0.078 & 242383 & 42.858 & 43.013 \\ 3 & 44.033 & \text { PV } & 0.060 & 198235 & 43.943 & 44.099 \\ 4 & 45.584 & \text { VV } & 0.057 & 768259 & 45.523 & 45.703 \\ 5 & 46.724 & \text { PV } & 0.065 & 516520 & 46.605 & 46.782 \\ 6 & 48.243 & \text { PV } & 0.093 & 397277 & 48.132 & 48.393 \\ 7 & 48.434 & \text { VV } & 0.054 & 256254 & 48.393 & 48.536 \\ 8 & 49.607 & \text { VV } & 0.076 & 194373 & 49.541 & 49.763\end{array}$


File:

operator:

Date Acquired:

Method File:

Sample Name:

Misc Info:

ALS vial:
C: \CHEMPC \DATA \DJB96H.D

BOYE

23 Jul 94 1:32 pm

JAFFE.M

ARABIAN CRUDE 96 HRS WEATHERING

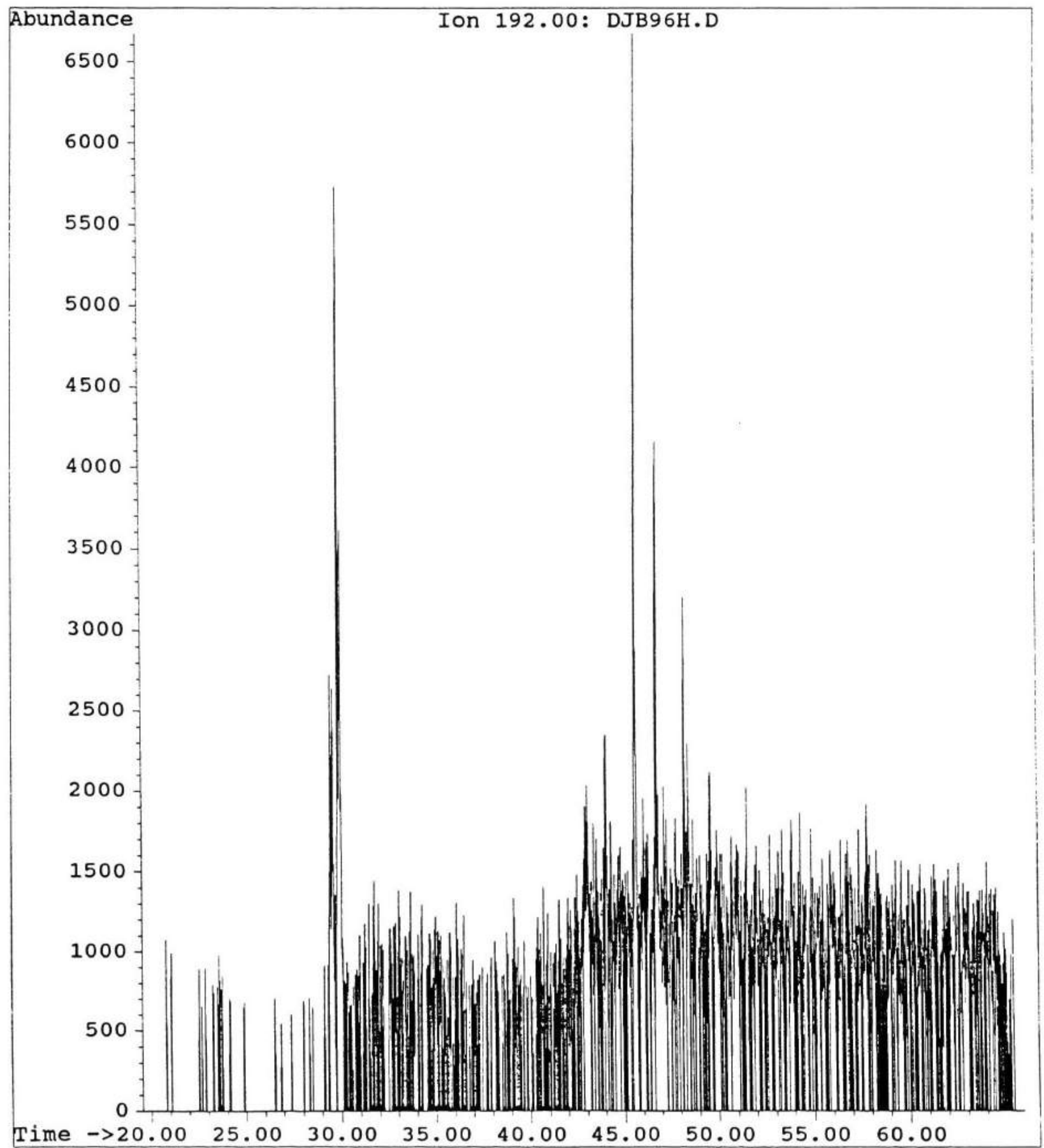


Ion 192.00: DJB96H.D

ARABIAN CRUDE 96 HRS WEATHERING

$\begin{array}{crlrr}\text { Peak\# } & \text { Ret Time } & \text { Type } & \text { Width } & \text { Area } \\ 1 & 29.421 & \text { BV } & 0.060 & 95076 \\ 2 & 29.521 & \text { VV } & 0.109 & 191196 \\ 3 & 29.815 & \text { PV } & 0.068 & 273944 \\ 4 & 29.940 & \text { VV } & 0.100 & 254035 \\ 5 & 42.929 & \text { VV } & 0.094 & 135446 \\ 6 & 43.015 & \text { VV } & 0.052 & 62723 \\ 7 & 43.906 & \text { VV } & 0.063 & 75924 \\ 8 & 44.021 & \text { VV } & 0.087 & 144386 \\ 9 & 44.282 & \text { VV } & 0.053 & 70908 \\ 10 & 45.586 & \text { VV } & 0.077 & 362964 \\ 11 & 46.245 & \text { VV } & 0.035 & 34449 \\ 12 & 46.724 & \text { VV } & 0.062 & 187058 \\ 13 & 48.233 & \text { VV } & 0.062 & 135768 \\ 14 & 48.436 & \text { VV } & 0.059 & 95560 \\ 15 & 49.383 & \text { VV } & 0.063 & 50151 \\ 16 & 49.594 & \text { VV } & 0.062 & 98632 \\ 17 & 49.811 & \text { PV } & 0.062 & 20070 \\ 18 & 50.203 & \text { PV } & 0.083 & 49098 \\ 19 & 51.494 & \text { VV } & 0.064 & 79269 \\ 20 & 55.841 & \text { PV } & 0.019 & 16705 \\ 21 & 57.941 & \text { PV } & 0.061 & 60220 \\ 22 & 62.028 & \text { VV } & 0.027 & 22149\end{array}$

$\begin{array}{rr}\text { Start Time } & \text { End Time } \\ 29.358 & 29.462 \\ 29.462 & 29.650 \\ 29.737 & 29.883 \\ 29.883 & 30.103 \\ 42.802 & 42.976 \\ 42.976 & 43.039 \\ 43.817 & 43.942 \\ 43.942 & 44.114 \\ 44.243 & 44.345 \\ 45.509 & 45.776 \\ 46.213 & 46.263 \\ 46.664 & 46.776 \\ 48.193 & 48.306 \\ 48.391 & 48.488 \\ 49.350 & 49.450 \\ 49.508 & 49.622 \\ 49.728 & 49.845 \\ 50.088 & 50.254 \\ 51.453 & 51.575 \\ 55.820 & 55.852 \\ 57.892 & 58.005 \\ 62.008 & 62.055\end{array}$


File:

Operator:

BOYE

Method File:

Sample Name:

Misc Info:

ALS vial:
L: \CHEMPC \DATA \DJB96H.D

JAFFE.M

1:32 pm

ARABIAN CRUDE 96 HRS WEATHERING

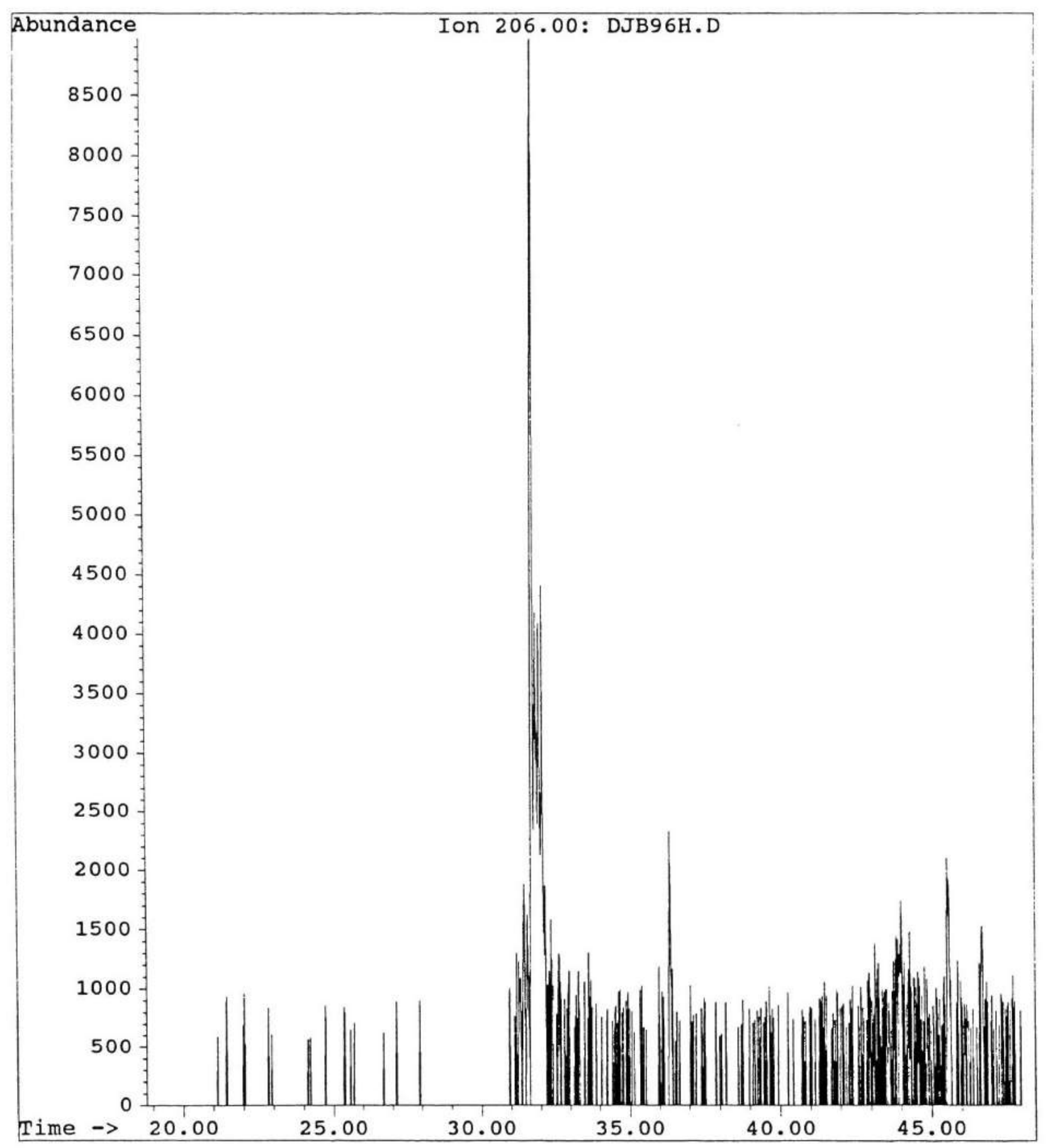


Ion $206.00:$ DJB96H.D

ARABIAN CRUDE 96 HRS WEATHERING

$\begin{array}{crlrrrr}\text { Peak\# } & \text { Ret Time } & \text { Type } & \text { width } & \text { Area } & \text { Start Time } & \text { End Time } \\ 1 & 31.703 & \text { PV } & 0.062 & 319817 & 31.629 & 31.775 \\ 2 & 31.839 & \text { VV } & 0.042 & 43715 & 31.775 & 31.861\end{array}$


281 
File:

operator:

Date Acquired:

Method File:

Sample Name:

Misc Info:

ALS vial:
C: \CHEMPC \DATA $\backslash$ DJB7DARB.D

boye

13 Aug 94 12:39 pm

JAFFE.M

1

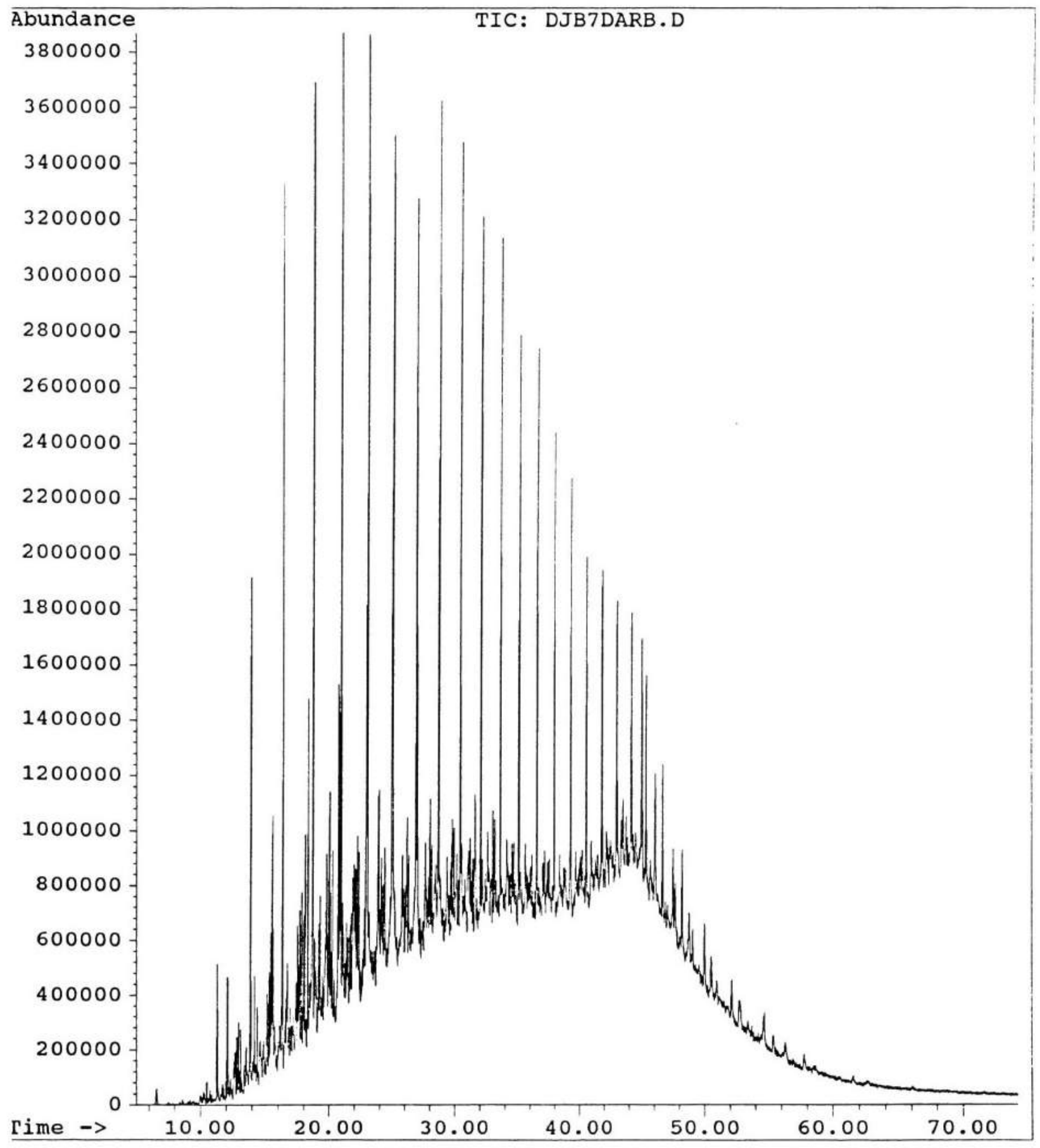


Pile:

Jperator:

Jate Acquired: 13 Aug $94 \quad 12: 39 \mathrm{pm}$

lethod File:

jample Name:

lisc Info:

ILS vial:

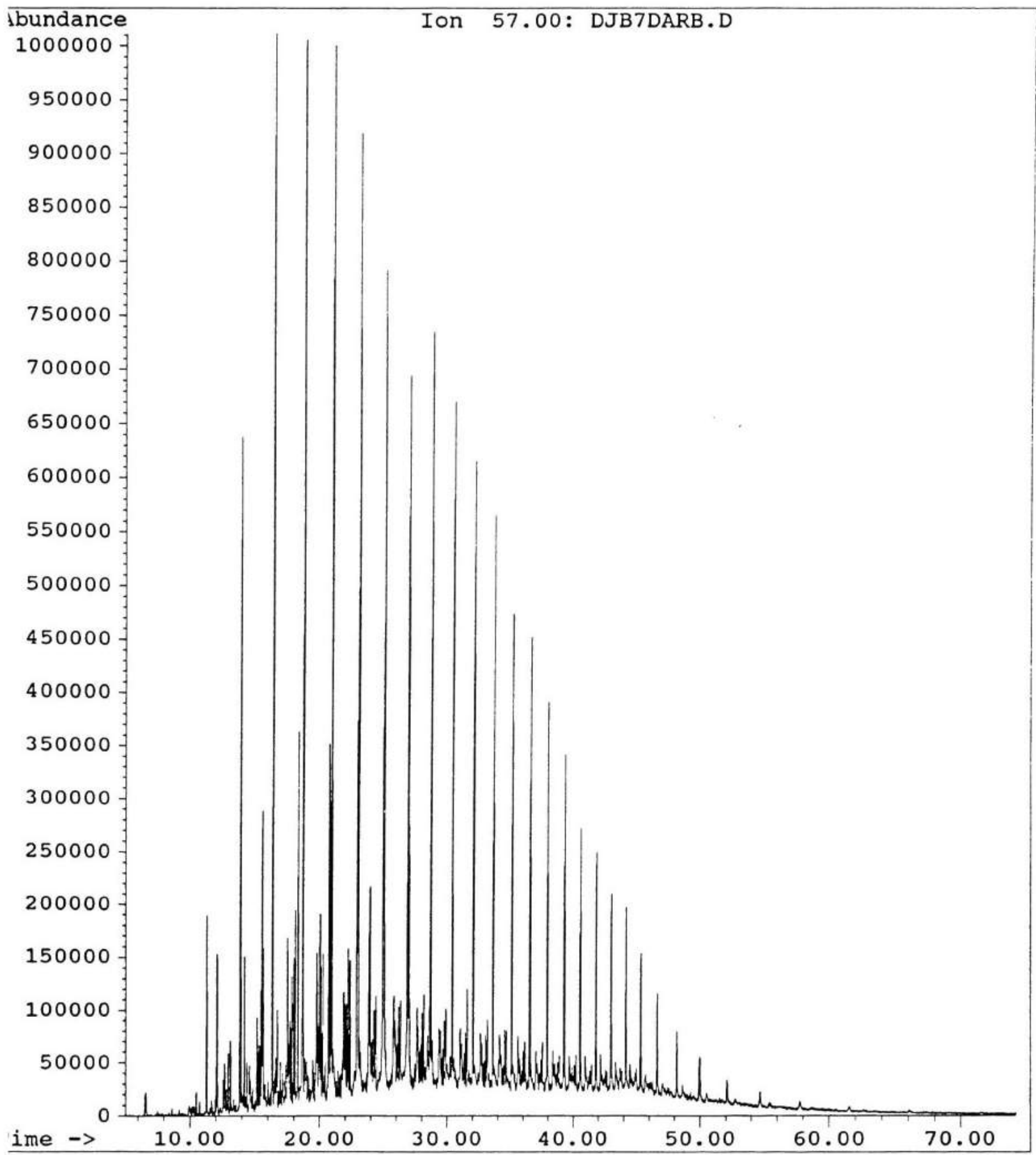




\begin{tabular}{|c|c|c|c|c|}
\hline Peak\# & Ret Time & Type & width & Area \\
\hline 1 & 11.271 & $\mathrm{BB}$ & 0.037 & 4296015 \\
\hline 2 & 12.072 & PV & 0.047 & 4296882 \\
\hline 3 & 12.659 & VV & 0.055 & 1770197 \\
\hline 4 & 12.940 & VV & 0.043 & 1413713 \\
\hline 5 & 13.101 & PV & 0.037 & 1573086 \\
\hline 6 & 13.877 & VV & 0.040 & 16016418 \\
\hline 7 & 14.183 & VV & 0.041 & 3778517 \\
\hline 8 & 14.389 & VV & 0.054 & 1544800 \\
\hline 9 & 14.586 & PV & 0.053 & 1520146 \\
\hline 10 & 15.146 & vV & 0.041 & 2339275 \\
\hline 11 & 15.322 & VV & 0.042 & 1602747 \\
\hline 12 & 15.459 & VV & 0.039 & 2732694 \\
\hline 13 & 15.545 & VV & 0.042 & 7317932 \\
\hline 14 & 15.624 & VV & 0.063 & 5997911 \\
\hline 15 & 16.362 & VV & 0.044 & 26825501 \\
\hline 16 & 16.590 & VV & 0.050 & 1532168 \\
\hline 17 & 16.726 & VV & 0.049 & 2866902 \\
\hline 18 & 16.970 & vV & 0.048 & 1327608 \\
\hline 19 & 17.514 & VV & 0.042 & 4138366 \\
\hline 20 & 17.583 & vV & 0.042 & 1537744 \\
\hline 21 & 17.649 & vV & 0.038 & 1389684 \\
\hline 22 & 17.706 & VV & 0.041 & 2130480 \\
\hline 23 & 17.846 & VV & 0.046 & 3519323 \\
\hline 24 & 17.997 & VV & 0.042 & 3729784 \\
\hline 25 & 18.107 & VV & 0.041 & 4800140 \\
\hline 26 & 18.318 & VV & 0.042 & 8845712 \\
\hline 27 & 18.710 & vV & 0.047 & 32258297 \\
\hline 28 & 19.770 & PV & 0.061 & 5886510 \\
\hline 29 & 19.842 & VV & 0.055 & 2660591 \\
\hline 30 & 20.022 & VV & 0.053 & 6662693 \\
\hline 31 & 20.105 & VV & 0.043 & 3303843 \\
\hline 32 & 20.251 & VV & 0.044 & 4012537 \\
\hline 33 & 20.726 & VV & 0.041 & 8807412 \\
\hline 34 & 20.926 & VV & 0.050 & 31909441 \\
\hline 35 & 21.898 & VV & 0.071 & 5060543 \\
\hline 36 & 21.984 & VV & 0.050 & 2798401 \\
\hline 37 & 22.113 & VV & 0.045 & 2479937 \\
\hline 38 & 22.242 & VV & 0.049 & 4327158 \\
\hline 39 & 22.383 & VV & 0.039 & 2890708 \\
\hline 40 & 22.918 & PV & 0.043 & 9130377 \\
\hline 41 & 23.019 & VV & 0.047 & 24818421 \\
\hline 42 & 23.931 & PV & 0.088 & 12368846 \\
\hline 43 & 24.013 & VV & 0.038 & 1177562 \\
\hline 44 & 24.264 & vV & 0.056 & 3090295 \\
\hline 45 & 24.405 & vV & 0.041 & 2303161 \\
\hline 46 & 25.005 & vV & 0.071 & 40021594 \\
\hline 47 & 25.793 & vV & 0.069 & 4356975 \\
\hline 48 & 25.861 & vV & 0.045 & 1690398 \\
\hline 49 & 26.183 & vV & 0.053 & 2781934 \\
\hline 50 & 26.324 & vV & 0.057 & 3271339 \\
\hline 51 & 26.888 & vV & 0.063 & 28409131 \\
\hline 52 & 26.983 & vV & 0.065 & 10565421 \\
\hline 53 & 27.618 & vV & 0.069 & 3553117 \\
\hline 54 & 27.691 & vV & 0.045 & 1492804 \\
\hline 55 & 28.012 & vV & 0.048 & 2101899 \\
\hline 56 & 28.152 & VV & 0.047 & 2612160 \\
\hline
\end{tabular}

$\begin{array}{rr}\text { Start Time } & \text { End Time } \\ 11.100 & 11.349 \\ 11.988 & 12.131 \\ 12.613 & 12.755 \\ 12.858 & 13.032 \\ 13.032 & 13.154 \\ 13.748 & 13.930 \\ 14.126 & 14.242 \\ 14.335 & 14.450 \\ 14.450 & 14.619 \\ 15.043 & 15.176 \\ 15.242 & 15.374 \\ 15.374 & 15.495 \\ 15.495 & 15.584 \\ 15.584 & 15.691 \\ 16.229 & 16.450 \\ 16.551 & 16.637 \\ 16.637 & 16.787 \\ 16.899 & 17.013 \\ 17.454 & 17.553 \\ 17.553 & 17.616 \\ 17.616 & 17.675 \\ 17.675 & 17.782 \\ 17.782 & 17.908 \\ 17.908 & 18.052 \\ 18.052 & 18.164 \\ 18.254 & 18.391 \\ 18.549 & 18.796 \\ 19.625 & 19.810 \\ 19.810 & 19.918 \\ 19.918 & 20.064 \\ 20.064 & 20.177 \\ 20.177 & 20.327 \\ 20.655 & 20.773 \\ 20.773 & 20.997 \\ 21.805 & 21.944 \\ 21.944 & 22.061 \\ 22.061 & 22.170 \\ 22.170 & 22.316 \\ 22.316 & 22.457 \\ 22.808 & 22.951 \\ 22.951 & 23.076 \\ 23.712 & 23.981 \\ 23.981 & 24.046 \\ 24.174 & 24.313 \\ 24.372 & 24.455 \\ 24.756 & 25.146 \\ 25.670 & 25.840 \\ 25.840 & 25.891 \\ 26.113 & 26.240 \\ 26.240 & 26.425 \\ 26.648 & 26.931 \\ 26.931 & 27.159 \\ 27.511 & 27.668 \\ 27.668 & 27.736 \\ 27.954 & 28.077 \\ 28.077 & 28.219 \\ & \end{array}$




$\begin{array}{lrlrrrr}57 & 28.452 & \text { VV } & 0.114 & 2965925 & 28.340 & 28.562 \\ 58 & 28.686 & \text { VV } & 0.048 & 21755074 & 28.562 & 28.739 \\ 59 & 29.357 & \text { VV } & 0.070 & 2639270 & 29.288 & 29.419 \\ 60 & 29.447 & \text { VV } & 0.040 & 1369386 & 29.419 & 29.491 \\ 61 & 29.756 & \text { PV } & 0.046 & 1733742 & 29.702 & 29.837 \\ 62 & 29.896 & \text { VV } & 0.044 & 1903203 & 29.837 & 29.944 \\ 63 & 30.402 & \text { PV } & 0.044 & 17896061 & 30.305 & 30.492 \\ 64 & 31.022 & \text { BV } & 0.060 & 2159044 & 30.818 & 31.057 \\ 65 & 31.421 & \text { VV } & 0.043 & 1391436 & 31.365 & 31.495 \\ 66 & 31.558 & \text { PV } & 0.050 & 3003241 & 31.495 & 31.630 \\ 67 & 32.040 & \text { PV } & 0.042 & 15704535 & 31.953 & 32.119 \\ 68 & 32.613 & \text { BV } & 0.069 & 2095199 & 32.503 & 32.692 \\ 69 & 33.017 & \text { PV } & 0.046 & 1442854 & 32.965 & 33.090 \\ 70 & 33.157 & \text { VV } & 0.056 & 2359541 & 33.090 & 33.270 \\ 71 & 33.606 & \text { PV } & 0.044 & 14207162 & 33.520 & 33.659 \\ 72 & 34.140 & \text { VV } & 0.066 & 2045277 & 34.092 & 34.232 \\ 73 & 34.540 & \text { PV } & 0.044 & 1453737 & 34.490 & 34.625 \\ 74 & 34.674 & \text { PV } & 0.040 & 1146374 & 34.625 & 34.726 \\ 75 & 35.107 & \text { PV } & 0.043 & 11992525 & 35.019 & 35.157 \\ 76 & 35.603 & \text { BV } & 0.064 & 2111263 & 35.415 & 35.693 \\ 77 & 36.548 & \text { PV } & 0.043 & 10913035 & 36.439 & 36.598 \\ 78 & 37.931 & \text { PV } & 0.039 & 8399762 & 37.859 & 37.979 \\ 79 & 39.266 & \text { BV } & 0.048 & 9540343 & 39.022 & 39.458 \\ 80 & 40.549 & \text { PV } & 0.046 & 7022370 & 40.429 & 40.667 \\ 81 & 41.789 & \text { VV } & 0.047 & 6726675 & 41.657 & 42.010 \\ 82 & 42.986 & \text { PV } & 0.045 & 4840428 & 42.748 & 43.050 \\ 83 & 44.144 & \text { PV } & 0.045 & 4818135 & 43.957 & 44.259 \\ 84 & 45.319 & \text { BV } & 0.052 & 4329478 & 45.074 & 45.483 \\ 85 & 46.644 & \text { VV } & 0.058 & 3465216 & 46.539 & 46.854 \\ 86 & 48.170 & \text { BB } & 0.066 & 2622461 & 47.996 & 48.415 \\ 87 & 49.969 & \text { PV } & 0.080 & 2221501 & 49.720 & 50.175\end{array}$


iile:

sperator:

late Acquired: 13 Aug $94 \quad 12: 39 \mathrm{pm}$

lethod File: JAFFE.M

iample Name:

Iisc Info:

ILS vial:

boye

1
C: \CHEMPC \DATA \DJB7DARB.D

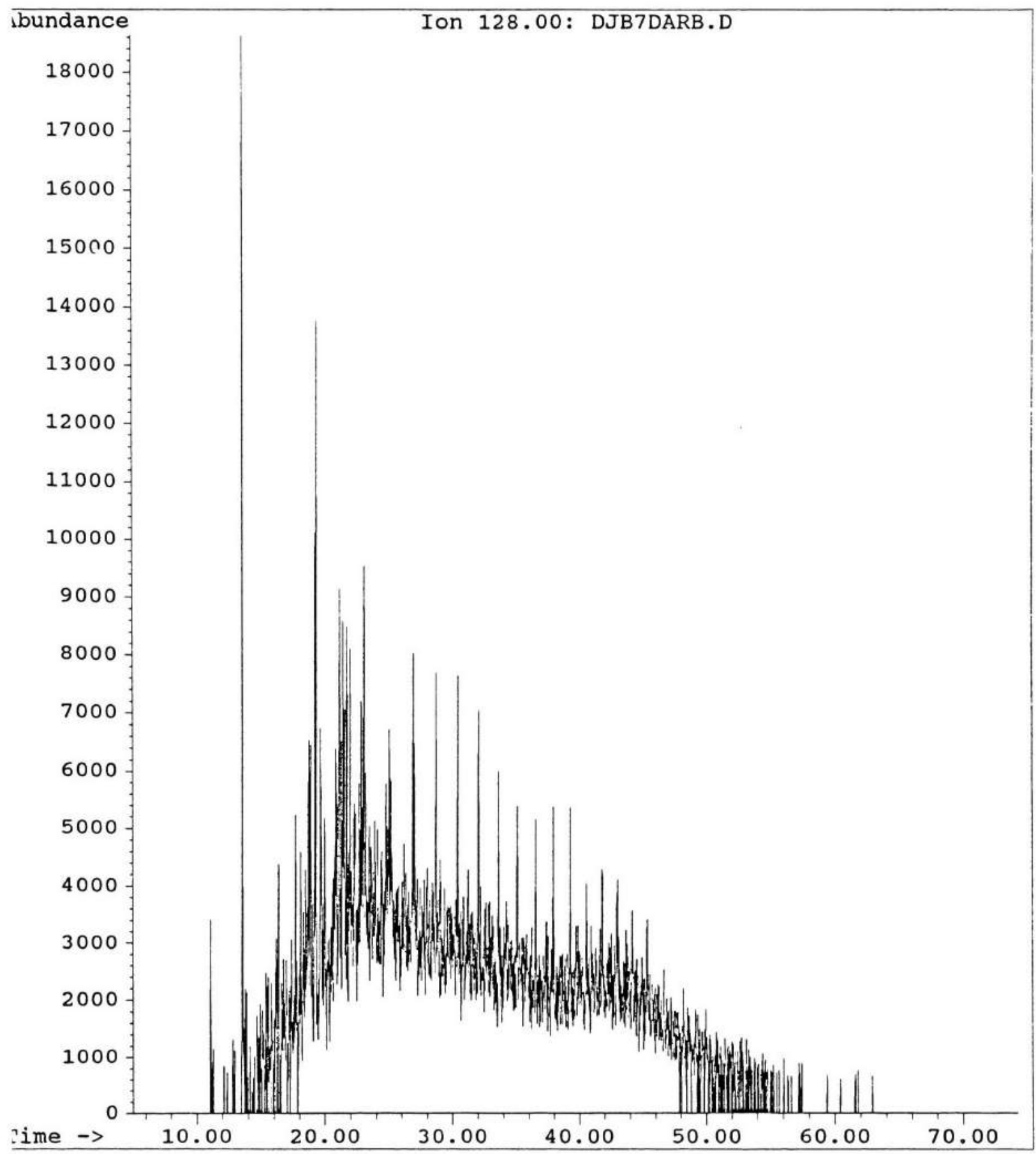


Ion $128.00:$ DJB7DARB.D

$\begin{array}{crllr}\text { Peak\# } & \text { Ret Time } & \text { Type } & \text { Width } & \text { Area } \\ 1 & 13.537 & \text { BV } & 0.050 & 617437 \\ 2 & 16.348 & \text { PV } & 0.067 & 189157 \\ 3 & 17.704 & \text { VV } & 0.045 & 137820 \\ 4 & 18.111 & \text { VV } & 0.060 & 150919 \\ 5 & 18.717 & \text { VV } & 0.068 & 253381 \\ 6 & 18.881 & \text { VV } & 0.058 & 230495 \\ 7 & 19.191 & \text { VV } & 0.054 & 323019 \\ 8 & 19.300 & \text { VV } & 0.055 & 455738 \\ 9 & 19.654 & \text { VV } & 0.108 & 378869 \\ 10 & 20.830 & \text { VV } & 0.071 & 197948 \\ 11 & 21.123 & \text { VV } & 0.079 & 415673 \\ 12 & 21.273 & \text { VV } & 0.058 & 155238 \\ 13 & 21.379 & \text { VV } & 0.044 & 176449 \\ 14 & 21.452 & \text { VV } & 0.045 & 110845 \\ 15 & 21.655 & \text { VV } & 0.051 & 189143 \\ 16 & 21.741 & \text { VV } & 0.048 & 147809 \\ 17 & 21.943 & \text { VV } & 0.045 & 166345 \\ 18 & 22.809 & \text { PV } & 0.040 & 105072 \\ 19 & 23.014 & \text { VV } & 0.054 & 229598 \\ 20 & 26.886 & \text { VV } & 0.051 & 149419 \\ 21 & 28.691 & \text { BV } & 0.049 & 126921 \\ 22 & 30.408 & \text { PV } & 0.048 & 118811 \\ 23 & 32.039 & \text { VV } & 0.064 & 196476 \\ 24 & 33.601 & \text { PV } & 0.052 & 115401\end{array}$

Start Time 13.422

16.294

17.635

18.066

18.643

18.799

19.105

19.245

19.542

20.768

20.997

21.192

21.323

21.414

21.588

21.694

21.897

22.761

22.964

26.828

28.572

30.333

31.940

33.514
End Time

13.688

16.456

17.757

18.237

18.799

18.911

19.245

19.416

19.806

20.879

21.192

21.323

21.414

21. 471

21.694

21.808

22.018

22.853

23.098

26.934

28.751

30.490

32.077

33.665 
File:

Operator:

Date Acquired: 13 Aug $94 \quad 12: 39 \mathrm{pm}$

Method File: JAFFE.M

Sample Name:

Misc Info:

ALS vial:
C: \CHEMPC \DATA \DJB7DARB.D

boye

1

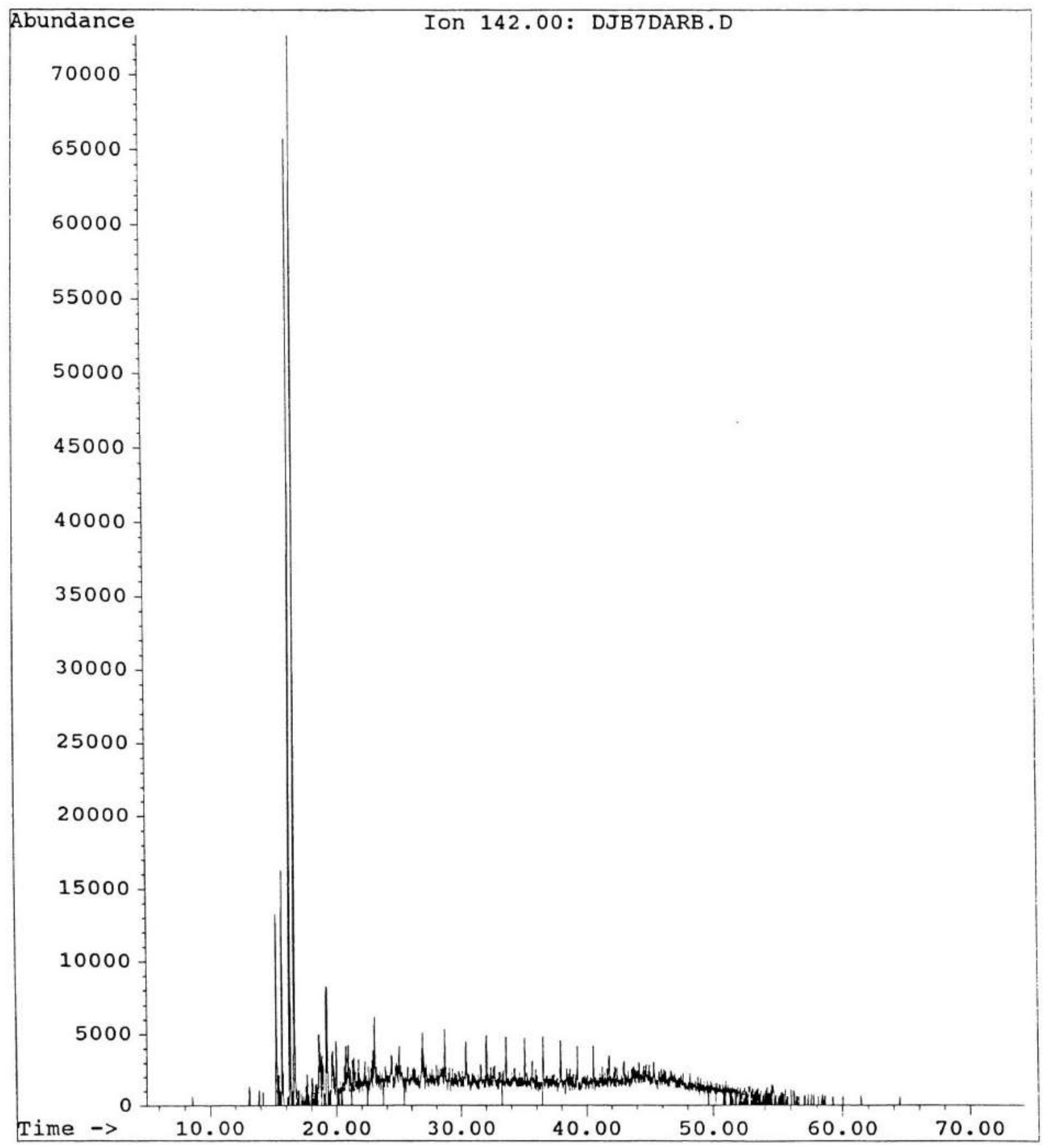


Ion $142.00:$ DJB7DARB.D

$\begin{array}{crlrrrr}\text { Peak\# } & \text { Ret Time } & \text { Type } & \text { width } & \text { Area } & \text { Start Time } & \text { End Time } \\ 1 & 15.244 & \text { BV } & 0.061 & 522345 & 15.192 & 15.411 \\ 2 & 15.702 & \text { BB } & 0.054 & 571220 & 15.639 & 15.830 \\ 3 & 16.309 & \text { BV } & 0.045 & 1872258 & 16.239 & 16.443 \\ 4 & 16.675 & \text { PV } & 0.046 & 2108711 & 16.443 & 16.823 \\ 5 & 19.192 & \text { PV } & 0.044 & 202485 & 19.118 & 19.243\end{array}$




\section{File:}

operator:

Date Acquired: 13 Aug $94 \quad 12: 39 \mathrm{pm}$

Method File:

Sample Name:

Misc Info:

ALS vial:

JAFFE.M

1

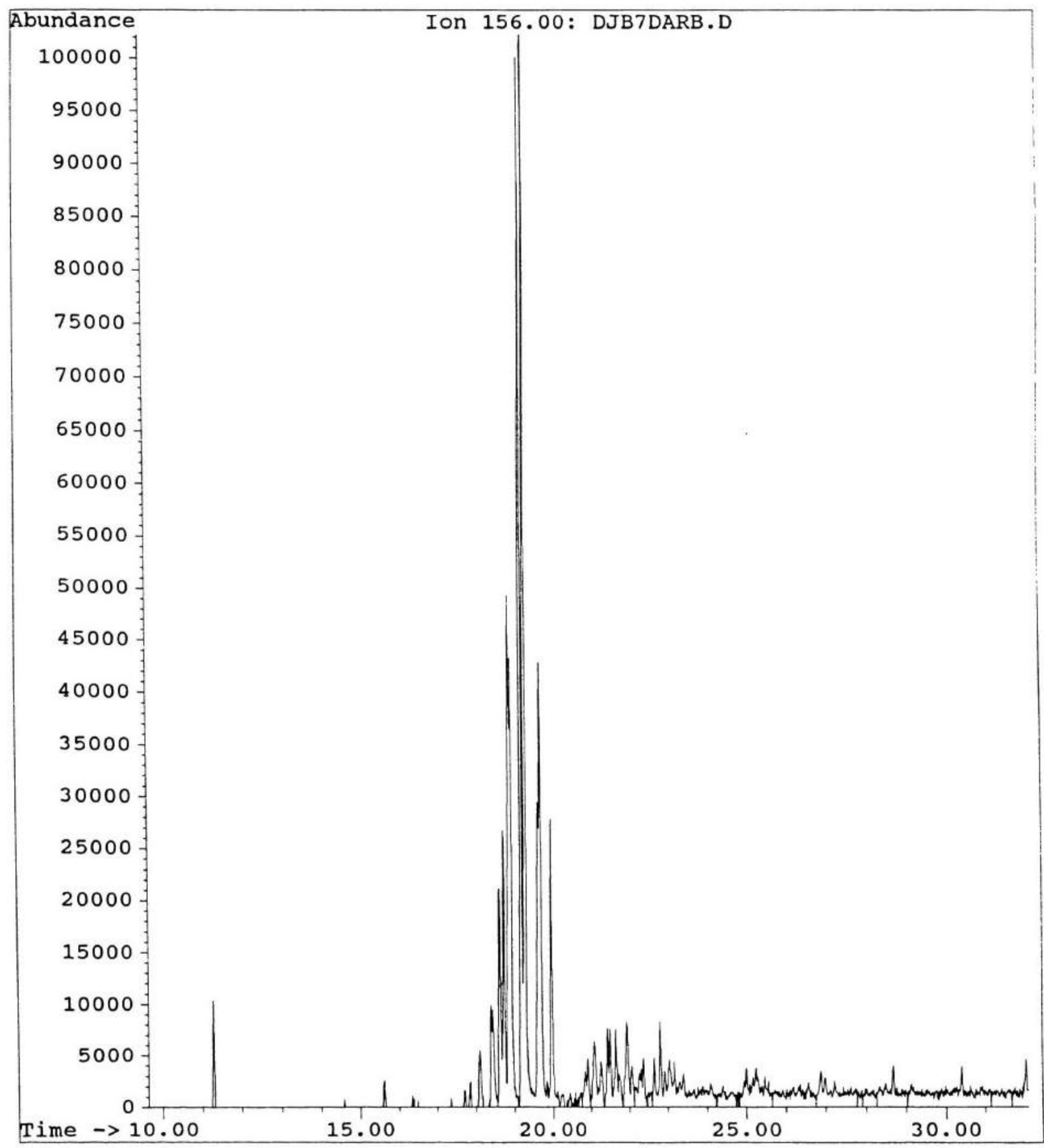


Ion $156.00:$ DJB7DARB'.D

$\begin{array}{crlrrrr}\text { Peak\# } & \text { Ret Time } & \text { Type } & \text { Width } & \text { Area } & \text { Start Time } & \text { End Time } \\ 1 & 11.273 & \text { BB } & 0.035 & 232331 & 11.224 & 11.319 \\ 2 & 18.398 & \text { BV } & 0.092 & 544147 & 18.351 & 18.559 \\ 3 & 18.622 & \text { PV } & 0.053 & 754206 & 18.559 & 18.685 \\ 4 & 18.734 & \text { VV } & 0.047 & 798331 & 18.685 & 18.799 \\ 5 & 18.887 & \text { VV } & 0.056 & 1887580 & 18.799 & 18.917 \\ 6 & 18.931 & \text { VB } & 0.039 & 952105 & 18.917 & 19.047 \\ 7 & 19.193 & \text { BV } & 0.049 & 2994638 & 19.075 & 19.244 \\ 8 & 19.293 & \text { VB } & 0.047 & 3091667 & 19.244 & 19.503 \\ 9 & 19.643 & \text { BV } & 0.058 & 967234 & 19.541 & 19.662 \\ 10 & 19.694 & \text { VV } & 0.047 & 1266480 & 19.662 & 19.807 \\ 11 & 19.967 & \text { PV } & 0.047 & 806206 & 19.880 & 20.092\end{array}$


File:

operator:

Date Acquired: 13 Aug $94 \quad 12: 39 \mathrm{pm}$

Method File:

Sample Name:

Misc Info:

ALS vial:
$C: \backslash C H E M P C \backslash D A T A \backslash D J B 7 D A R B . D$

boye

JAFFE.M

1

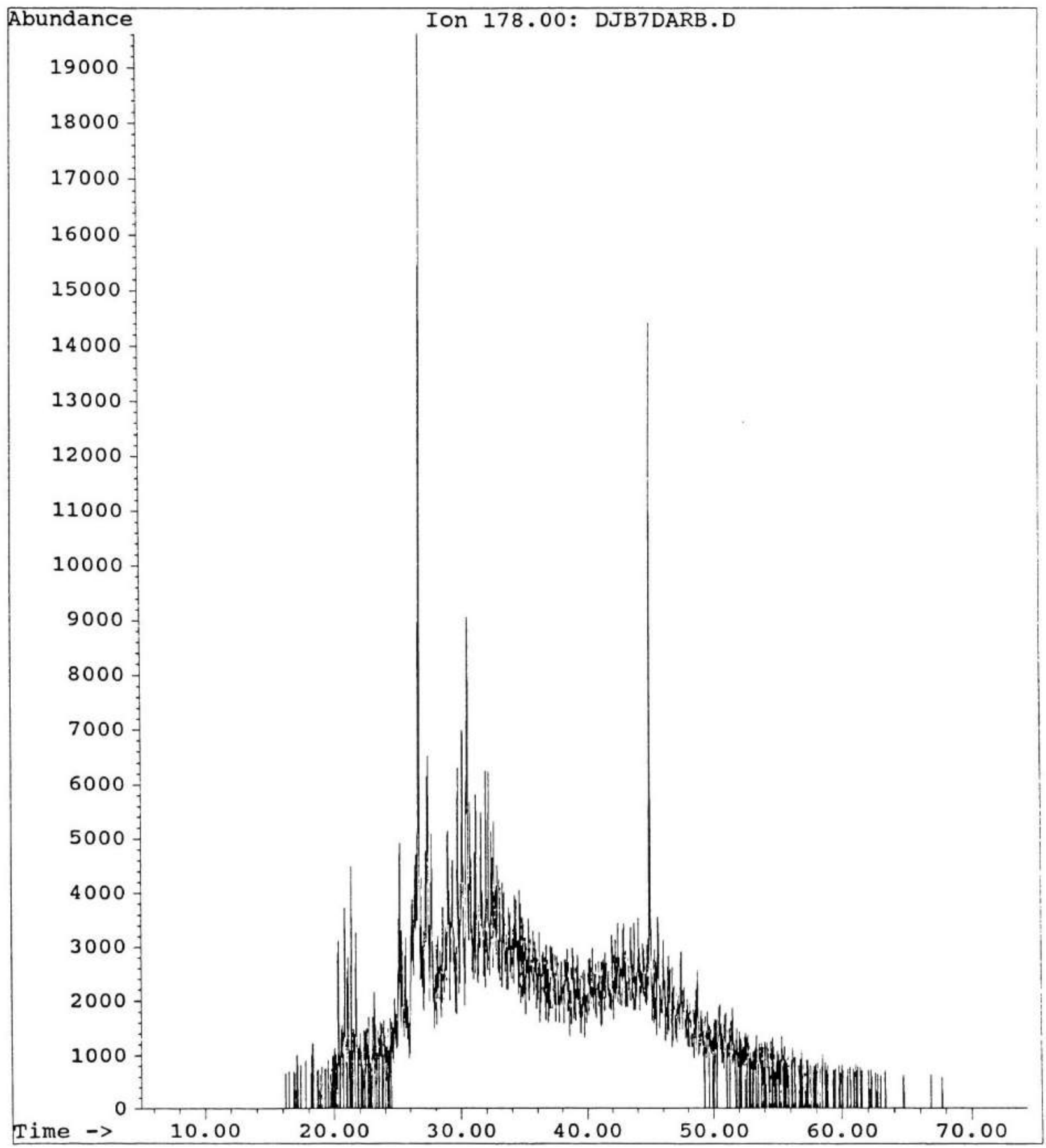


Ion 178.00 : DJB7DARB.D

$\begin{array}{crlrrrr}\text { Peak\# } & \text { Ret Time } & \text { Type } & \text { Width } & \text { Area } & \text { Start Time } & \text { End Time } \\ 1 & 20.874 & \text { VB } & 0.079 & 202925 & 20.746 & 20.988 \\ 2 & 21.378 & \text { PV } & 0.051 & 154232 & 21.267 & 21.425 \\ 3 & 25.198 & \text { PV } & 0.083 & 210632 & 24.930 & 25.272 \\ 4 & 26.702 & \text { VV } & 0.071 & 890468 & 26.588 & 26.829 \\ 5 & 27.413 & \text { VV } & 0.111 & 391402 & 27.302 & 27.545 \\ 6 & 29.751 & \text { PV } & 0.074 & 199936 & 29.656 & 29.797 \\ 7 & 30.115 & \text { VV } & 0.065 & 181737 & 30.028 & 30.173 \\ 8 & 30.511 & \text { PV } & 0.102 & 381603 & 30.338 & 30.563 \\ 9 & 31.968 & \text { PV } & 0.049 & 119262 & 31.913 & 32.089 \\ 10 & 32.172 & \text { VV } & 0.062 & 152060 & 32.089 & 32.271 \\ 11 & 44.983 & \text { VV } & 0.064 & 522778 & 44.901 & 45.166\end{array}$


File:

operator:

Date Acquired:

Method File:

Sample Name:

Misc Info:

ALS vial:
C: \CHEMPC \DATA \DJB7DARB.D

boye

13 Aug 94 12:39 pm

JAFFE.M

1

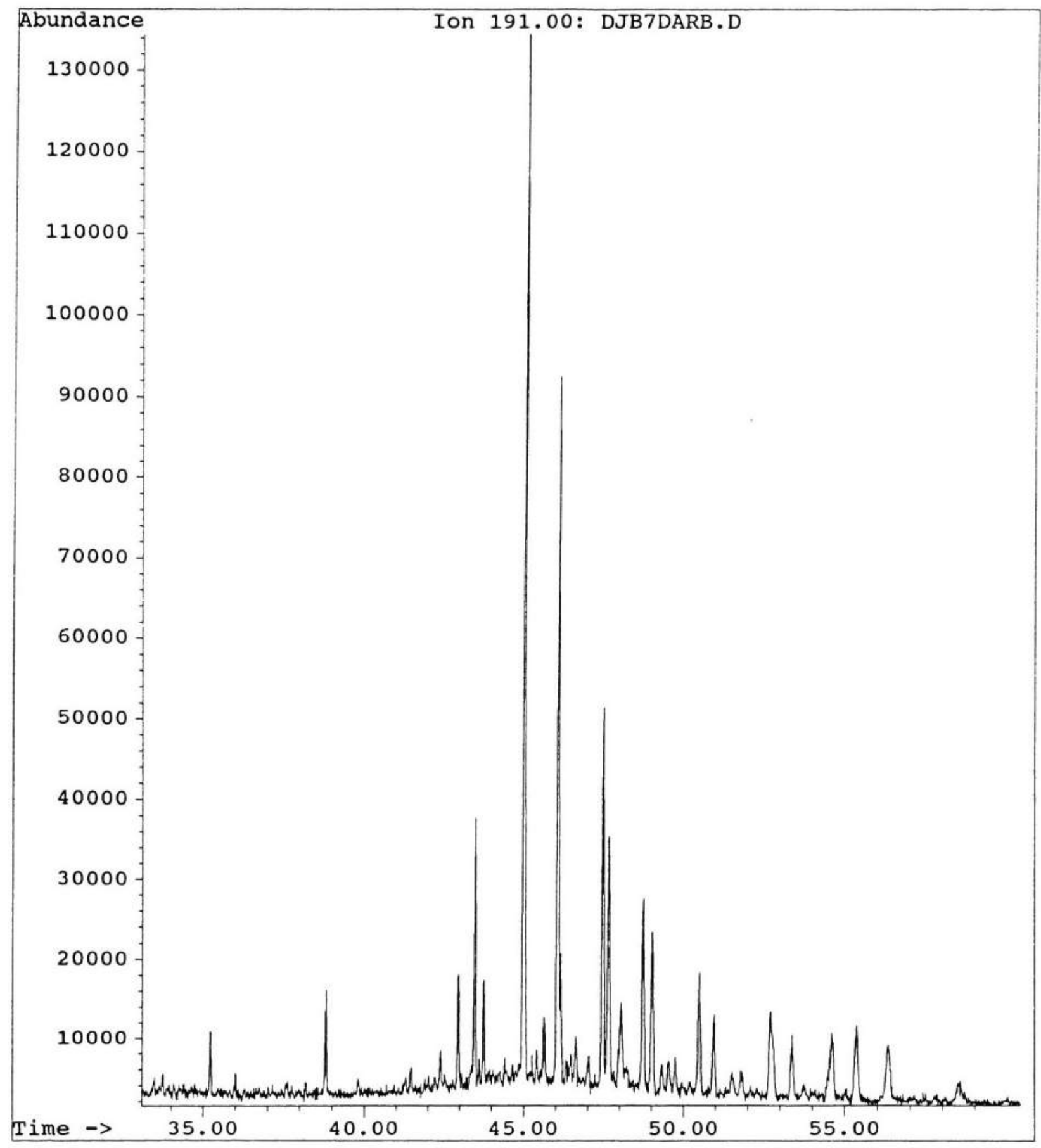


Ion 191.00: DJB7DARB.D

$\begin{array}{crlrrrr}\text { Peak\# } & \text { Ret Time } & \text { Type } & \text { Width } & \text { Area } & \text { Start Time } & \text { End Time } \\ 1 & 29.172 & \text { PV } & 0.052 & 417570 & 29.101 & 29.229 \\ 2 & 31.083 & \text { BV } & 0.048 & 400729 & 30.961 & 31.163 \\ 3 & 38.805 & \text { BV } & 0.060 & 390784 & 38.660 & 38.926 \\ 4 & 42.944 & \text { PV } & 0.053 & 509787 & 42.759 & 43.020 \\ 5 & 43.467 & \text { VV } & 0.048 & 1035378 & 43.391 & 43.536 \\ 6 & 43.739 & \text { BV } & 0.044 & 371609 & 43.666 & 43.813 \\ 7 & 44.989 & \text { VV } & 0.056 & 4817800 & 44.815 & 45.109 \\ 8 & 46.057 & \text { VV } & 0.058 & 3256172 & 45.940 & 46.120 \\ 9 & 46.151 & \text { VB } & 0.054 & 565074 & 46.120 & 46.273 \\ 10 & 47.470 & \text { BV } & 0.061 & 1882856 & 47.358 & 47.554 \\ 11 & 47.651 & \text { VV } & 0.075 & 1465038 & 47.554 & 47.777 \\ 12 & 48.744 & \text { PV } & 0.081 & 1297724 & 48.471 & 48.852 \\ 13 & 49.025 & \text { VV } & 0.070 & 984739 & 48.902 & 49.148 \\ 14 & 50.498 & \text { VB } & 0.071 & 783332 & 50.365 & 50.642\end{array}$


File:

Operator:

Date Acquired: 13 Au

Method File: JAFFE.M

Sample Name:

Misc Info:

ALS vial:

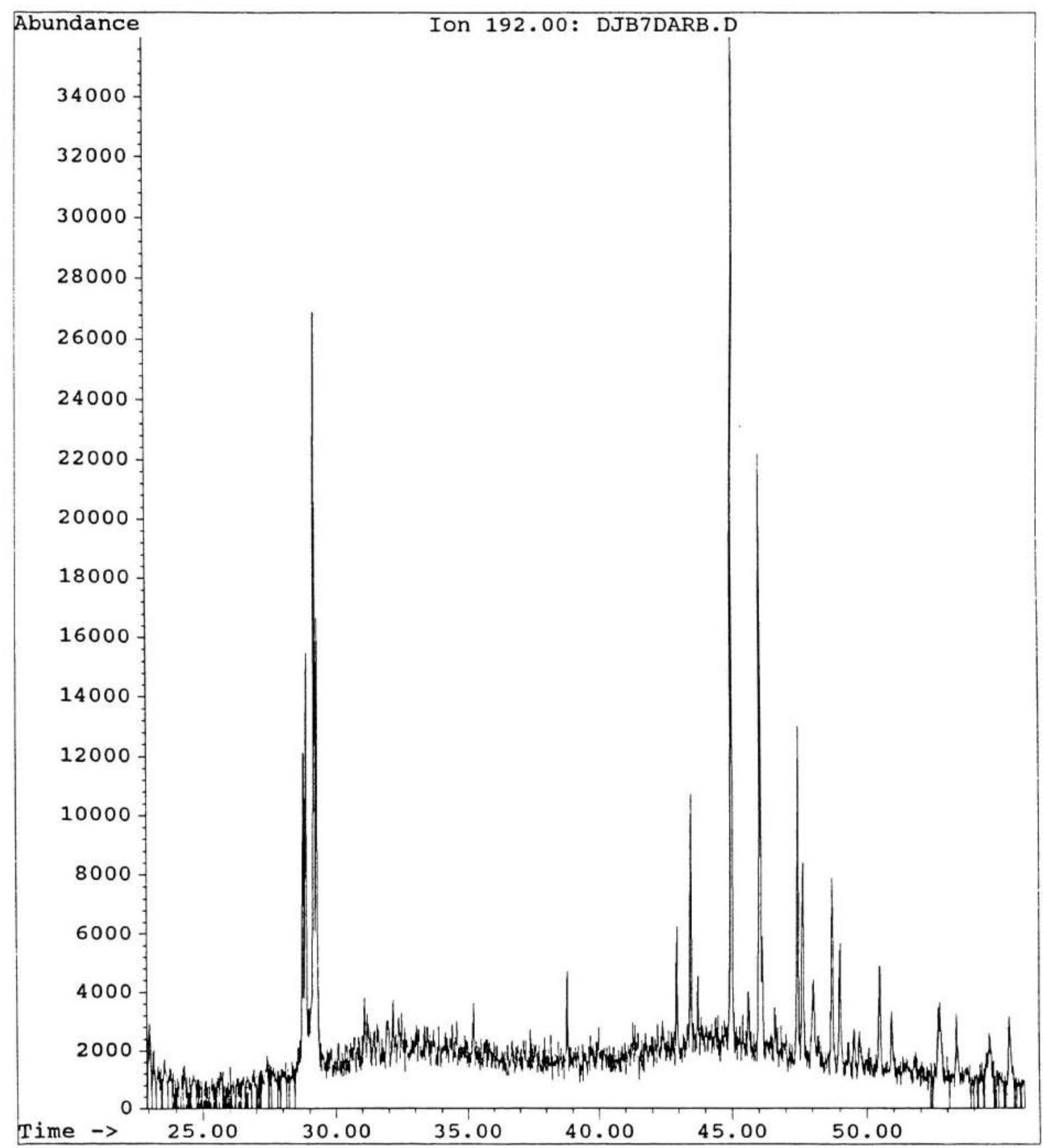


Ion 192.00: DJB7DARB.D

$\begin{array}{crlrrrr}\text { Peak\# } & \text { Ret Time } & \text { Type } & \text { Width } & \text { Area } & \text { Start Time } & \text { End Time } \\ 1 & 28.766 & \text { VV } & 0.058 & 415814 & 28.685 & 28.811 \\ 2 & 28.873 & \text { VV } & 0.060 & 552639 & 28.811 & 28.965 \\ 3 & 29.174 & \text { VV } & 0.060 & 1039437 & 29.044 & 29.224 \\ 4 & 29.281 & \text { VV } & 0.061 & 639308 & 29.224 & 29.448 \\ 5 & 42.947 & \text { PV } & 0.052 & 146754 & 42.873 & 43.032 \\ 6 & 43.463 & \text { VV } & 0.052 & 276052 & 43.394 & 43.542 \\ 7 & 44.989 & \text { PV } & 0.049 & 1107513 & 44.863 & 45.087 \\ 8 & 46.054 & \text { PV } & 0.051 & 604067 & 45.942 & 46.115 \\ 9 & 47.471 & \text { PV } & 0.058 & 463639 & 47.346 & 47.565 \\ 10 & 47.661 & \text { VV } & 0.075 & 364256 & 47.565 & 47.747 \\ 11 & 48.732 & \text { PV } & 0.067 & 330344 & 48.629 & 48.903 \\ 12 & 49.041 & \text { VV } & 0.094 & 237977 & 48.903 & 49.164\end{array}$


File:

operator:

Date Acquired:

Method File:

Sample Name:

Misc Info:

ALS vial:
C: \CHEMPC \DATA \DJB7DARB.D

boye

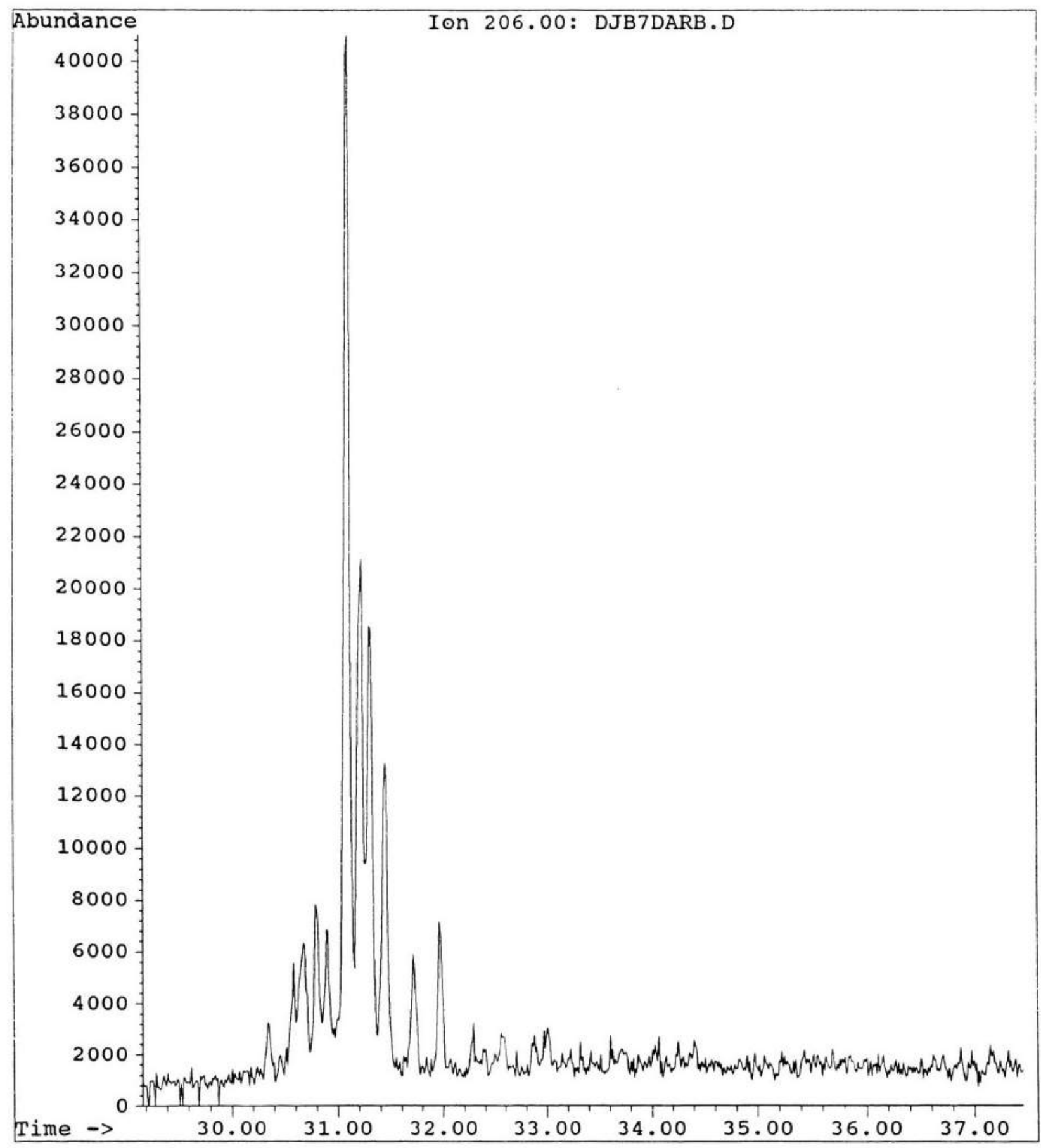


Ion 206.00: DJB7DARB.D

$\begin{array}{crlrrrr}\text { Peak\# } & \text { Ret Time } & \text { Type } & \text { Width } & \text { Area } & \text { Start Time } & \text { End Time } \\ 1 & 31.084 & \text { VV } & 0.060 & 1591054 & 30.972 & 31.155 \\ 2 & 31.216 & \text { VV } & 0.065 & 838184 & 31.155 & 31.260 \\ 3 & 31.302 & \text { VV } & 0.058 & 662015 & 31.260 & 31.373 \\ 4 & 31.448 & \text { VV } & 0.064 & 510133 & 31.373 & 31.603 \\ 5 & 44.964 & \text { VV } & 0.077 & 435266 & 44.793 & 45.169\end{array}$


9.10 APPENDIX J

SOLUBLE AROMATIC HYDROCARBONS DETECTED IN WEATHERING FLASK BELOW CRUDE OIL SLICK, AS DETERMINED BY FLUORESCENCE SPECTROMETRY 
Total Aromatics Measured in Aqueous Phase vs. Weathering Period

\begin{tabular}{|c|r|}
\hline Weathering Period ( hours ) & Recorded Concentration (ppm ) \\
\hline unweathered crude & N/A \\
\hline 4 & $* 0.449$ \\
\hline 4 & 0.181 \\
\hline 8 & 0.146 \\
\hline 8 & 0.151 \\
\hline 24 & 0.129 \\
\hline 24 & 0.156 \\
\hline 96 & 0.174 \\
\hline 96 & 0.228 \\
\hline 168 & 0.066 \\
\hline 168 & 0.049 \\
\hline
\end{tabular}

* data point excluded from data plot: considered an outlier; possible contamination

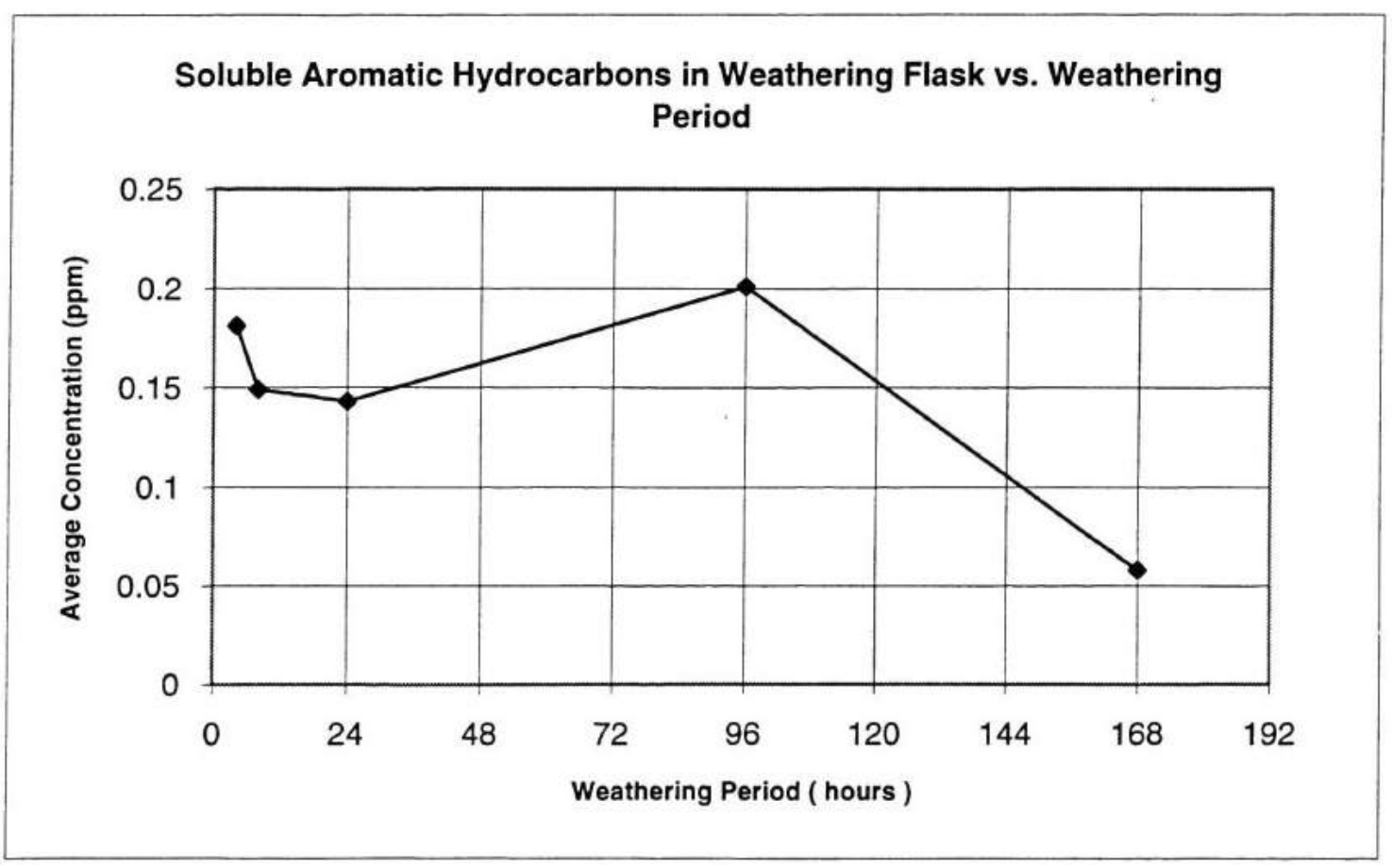




\subsection{APPENDIX K}

TOTAL OIL CONCENTRATION PROFILE RECORDED WITHIN VERTICAL TURBULENT DISPERSION REACTOR FOR WEATHERED ARABIAN CRUDE OIL SAMPLES UNDER SIMULATED TURBULENCE 
Total Hydrocarbons Dispersed within Water Column vs. Turbulence Duration

Weathering Period

unweathered

\begin{tabular}{|c|c|c|c|}
\hline $\begin{array}{l}\text { Turbulence } \\
\text { Duration } \\
\text { (minutes ) }\end{array}$ & $\begin{array}{c}\text { Surface Concentration } \\
\text { depth = 3" } \\
(\mathrm{ppm})\end{array}$ & $\begin{array}{c}\text { Mid-level Concentration } \\
\text { depth = 27" } \\
(\mathrm{ppm})\end{array}$ & $\begin{array}{c}\text { Bottom Concentraion } \\
\text { depth = 63" } \\
(\mathrm{ppm})\end{array}$ \\
\hline \multirow[t]{2}{*}{5} & 1051.2 & 901.6 & 758.4 \\
\hline & 1053.6 & 951.2 & 708.8 \\
\hline \multirow[t]{2}{*}{180} & 865.6 & 754.4 & 720.8 \\
\hline & 843.2 & 764.8 & 813.6 \\
\hline \multirow[t]{2}{*}{180 post-shutdown } & 344 & 337.6 & 352 \\
\hline & 314 & 306.4 & N/A \\
\hline
\end{tabular}

Normalized Data Set

\begin{tabular}{|c|c|c|c|}
\hline $\begin{array}{c}\text { Turbulence } \\
\text { Duration } \\
\text { (minutes ) } \\
\end{array}$ & $\begin{array}{c}\text { Surface Concentration } \\
\text { depth }=3^{\prime \prime} \\
(\mathrm{ppm}) \\
\end{array}$ & $\begin{array}{c}\text { Mid-level Concentration } \\
\text { depth }=27^{\prime \prime} \\
(\mathrm{ppm})\end{array}$ & $\begin{array}{c}\text { Bottom Concentraion } \\
\text { depth }=63^{\prime \prime} \\
(\mathrm{ppm})\end{array}$ \\
\hline \multirow[t]{2}{*}{5} & 1058.5584 & 907.9112 & 763.7088 \\
\hline & 1060.9752 & 957.8584 & 713.7616 \\
\hline \multirow[t]{2}{*}{180} & 871.6592 & 759.6808 & 725.8456 \\
\hline & 849.1024 & 770.1536 & 819.2952 \\
\hline \multirow[t]{2}{*}{180 post-shutdown } & 346.408 & 339.9632 & 354.464 \\
\hline & 316.198 & 308.5448 & \\
\hline
\end{tabular}


Total Hydrocarbons Dispersed within Water Column vs. Turbulence Duration

Weathering Period

4 hours

\begin{tabular}{|c|c|c|c|}
\hline $\begin{array}{c}\text { Turbulence } \\
\text { Duration } \\
\text { (minutes })\end{array}$ & $\begin{array}{c}\text { Surface Concentration } \\
\text { depth=3" } \\
(\mathrm{ppm})\end{array}$ & $\begin{array}{c}\text { Mid-level Concentration } \\
\text { depth }=27^{\prime \prime} \\
(\mathrm{ppm})\end{array}$ & $\begin{array}{c}\text { Bottom Concentraion } \\
\text { depth =63" } \\
(\mathrm{ppm})\end{array}$ \\
\hline & 1063.2 & & 591.2 \\
\hline 5 & 1128 & 984.8 & 672.8 \\
\hline & & 912 & 765.6 \\
\hline 180 & 861.6 & 887.2 & 841.6 \\
\hline & 848 & 922.6 & 432 \\
\hline 180 post-shutdown & 320 & & 379.2 \\
\hline & 366.4 & 274.4 & 353.6 \\
\hline
\end{tabular}

Normalized Data Set

\begin{tabular}{|c|c|c|c|}
\hline $\begin{array}{l}\text { Turbulence } \\
\text { Duration } \\
\text { ( minutes ) }\end{array}$ & $\begin{array}{c}\text { Surface Concentration } \\
\text { depth }=3^{\prime \prime} \\
(\mathrm{ppm})\end{array}$ & $\begin{array}{c}\text { Mid-level Concentration } \\
\text { depth }=27 " \\
(\mathrm{ppm})\end{array}$ & $\begin{array}{c}\text { Bottom Concentraion } \\
\text { depth = 63" } \\
(\mathrm{ppm})\end{array}$ \\
\hline \multirow[t]{2}{*}{5} & 1174.836 & 1088.204 & 653.276 \\
\hline & 1246.44 & 1007.76 & 743.444 \\
\hline \multirow[t]{2}{*}{180} & 952.068 & 980.356 & 845.988 \\
\hline & 937.04 & 1019.473 & 929.968 \\
\hline \multirow[t]{2}{*}{180 post-shutdown } & 353.6 & 303.212 & 477.36 \\
\hline & 404.872 & 390.728 & 419.016 \\
\hline
\end{tabular}


Total Hydrocarbons Dispersed within Water Column vs. Turbulence Duration

Weathering Period

8 hours

\begin{tabular}{|c|c|c|c|}
\hline $\begin{array}{c}\text { Turbulence } \\
\text { Duration } \\
\text { (minutes })\end{array}$ & $\begin{array}{c}\text { Surface Concentration } \\
\text { depth }=3^{\prime \prime} \\
(\mathrm{ppm})\end{array}$ & $\begin{array}{c}\text { Mid-level Concentration } \\
\text { depth }=27^{\prime \prime} \\
(\mathrm{ppm})\end{array}$ & $\begin{array}{c}\text { Bottom Concentraion } \\
\text { depth }=63^{\prime \prime} \\
(\mathrm{ppm})\end{array}$ \\
\hline 5 & 1066.4 & & 620.8 \\
\hline & 1150.4 & 1259.2 & 658.4 \\
\hline & 1072.8 & & 846.4 \\
\hline 180 & 969.6 & 943.2 & 863.2 \\
\hline & & 985.6 & 398.4 \\
\hline 180 post-shutdown & 422.4 & 352 & N/A \\
\hline & 242.4 & 304.8 & \\
\hline
\end{tabular}

\begin{tabular}{|c|c|c|c|}
\hline $\begin{array}{c}\text { Turbulence } \\
\text { Duration } \\
\text { (minutes })\end{array}$ & $\begin{array}{c}\text { Surface Concentration } \\
\text { depth }=3^{\prime \prime} \\
(\mathrm{ppm})\end{array}$ & $\begin{array}{c}\text { Mid-level Concentration } \\
\text { depth }=27^{\prime \prime} \\
(\mathrm{ppm})\end{array}$ & $\begin{array}{c}\text { Bottom Concentraion } \\
\text { depth }=63^{\prime \prime} \\
(\mathrm{ppm})\end{array}$ \\
\hline & & & 681.6384 \\
\hline 5 & 1170.9072 & 1382.6016 & 722.9232 \\
\hline & 1263.1392 & 1397.5344 & 929.3472 \\
\hline & & & 947.7936 \\
\hline 180 & 1177.9344 & 1035.6336 & 437.4432 \\
\hline & 1064.6208 & 1082.1888 & \\
\hline & & & 386.496 \\
\hline
\end{tabular}


Total Hydrocarbons Dispersed within Water Column vs. Turbulence Duration

Weathering Period

1 day

\begin{tabular}{|c|c|c|c|}
\hline $\begin{array}{c}\text { Turbulence } \\
\text { Duration } \\
\text { (minutes })\end{array}$ & $\begin{array}{c}\text { Surface Concentration } \\
\text { depth }=3^{\prime \prime} \\
(\mathrm{ppm})\end{array}$ & $\begin{array}{c}\text { Mid-level Concentration } \\
\text { depth }=27^{\prime \prime} \\
(\mathrm{ppm})\end{array}$ & $\begin{array}{c}\text { Bottom Concentraion } \\
\text { depth }=63^{\prime \prime} \\
(\mathrm{ppm})\end{array}$ \\
\hline 5 & 1062.4 & 948.8 & 729.6 \\
\hline & 1063.2 & 1059.2 & 626.4 \\
\hline & & & 789.6 \\
\hline 180 & 917.6 & 902.4 & N/A \\
\hline & 1027.2 & 816.8 & 447.2 \\
\hline 180 post-shutdown & 372.8 & & 336.8 \\
\hline & 313.6 & 276 & 310.4 \\
\hline
\end{tabular}

Normalized Data Set

\begin{tabular}{|c|c|c|c|}
\hline $\begin{array}{c}\text { Turbulence } \\
\text { Duration } \\
\text { (minutes })\end{array}$ & $\begin{array}{c}\text { Surface Concentration } \\
\text { depth }=3^{\prime \prime} \\
(\mathrm{ppm})\end{array}$ & $\begin{array}{c}\text { Mid-level Concentration } \\
\text { depth }=27^{\prime \prime} \\
(\mathrm{ppm})\end{array}$ & $\begin{array}{c}\text { Bottom Concentraion } \\
\text { depth }=63^{\prime \prime} \\
(\mathrm{ppm})\end{array}$ \\
\hline & & & \\
\hline 5 & 1131.456 & 1010.472 & 777.024 \\
\hline & 1132.308 & 1128.048 & 667.116 \\
\hline 180 & & & 840.924 \\
\hline & 977.244 & 961.056 & \\
\hline & 1093.968 & 869.892 & 476.268 \\
\hline 180 post-shutdown & 397.032 & 293.94 & 358.692 \\
\hline
\end{tabular}


Total Hydrocarbons Dispersed within Water Column vs. Turbulence Duration

Weathering Period

4 days

\begin{tabular}{|c|c|c|c|}
\hline $\begin{array}{l}\text { Turbulence } \\
\text { Duration } \\
\text { (minutes ) } \\
\end{array}$ & $\begin{array}{c}\text { Surface Concentration } \\
\text { depth }=3^{\prime \prime} \\
(\mathrm{ppm})\end{array}$ & $\begin{array}{c}\text { Mid-level Concentration } \\
\text { depth }=27^{\prime \prime} \\
(\mathrm{ppm})\end{array}$ & $\begin{array}{c}\text { Bottom Concentraion } \\
\text { depth }=63^{\prime \prime} \\
(\mathrm{ppm})\end{array}$ \\
\hline \multirow[t]{2}{*}{5} & 1209.6 & 1243.2 & 605.6 \\
\hline & 1297.6 & 1086.4 & 621.6 \\
\hline \multirow{2}{*}{180} & 808.8 & 9568 & 549.6 \\
\hline & 1061.6 & 966.4 & 644.8 \\
\hline & & & \\
\hline \multirow[t]{2}{*}{180 post-shutdown } & 400.8 & 270.4 & 326.4 \\
\hline & 337.6 & 309.6 & 359.2 \\
\hline
\end{tabular}

Normalized Data Set

\begin{tabular}{|c|c|c|c|}
\hline $\begin{array}{c}\text { Turbulence } \\
\text { Duration } \\
\text { (minutes })\end{array}$ & $\begin{array}{c}\text { Surface Concentration } \\
\text { depth =3" } \\
(\mathrm{ppm})\end{array}$ & $\begin{array}{c}\text { Mid-level Concentration } \\
\text { depth }=27^{\prime \prime} \\
(\mathrm{ppm})\end{array}$ & $\begin{array}{c}\text { Bottom Concentraion } \\
\text { depth }=63^{\prime \prime} \\
(\mathrm{ppm})\end{array}$ \\
\hline & & & 601.3608 \\
\hline 5 & 1201.1328 & 1234.4976 & 617.2488 \\
\hline & 1288.5168 & 1078.7952 & 545.7528 \\
\hline 180 & 803.1384 & 950.1024 & 640.2864 \\
\hline & 1054.1688 & 959.6352 & 324.1152 \\
\hline 180 post-shutdown & 397.9944 & & 356.6856 \\
\hline & 335.2368 & 268.5072 & \\
\hline
\end{tabular}


Total Hydrocarbons Dispersed within Water Column vs. Turbulence Duration

Weathering Period

7 days

\begin{tabular}{|c|c|c|c|}
\hline $\begin{array}{c}\text { Turbulence } \\
\text { Duration } \\
\text { (minutes })\end{array}$ & $\begin{array}{c}\text { Surface Concentration } \\
\text { depth }=3^{\prime \prime} \\
(\mathrm{ppm})\end{array}$ & $\begin{array}{c}\text { Mid-level Concentration } \\
\text { depth }=27^{\prime \prime} \\
(\mathrm{ppm})\end{array}$ & $\begin{array}{c}\text { Bottom Concentraion } \\
\text { depth }=63^{\prime \prime} \\
(\mathrm{ppm})\end{array}$ \\
\hline 5 & 1931.2 & 1128.8 & 581.6 \\
\hline & 1750.4 & 1259.2 & 628.8 \\
\hline & & & 617.6 \\
\hline 180 & 948 & 893.6 & 572.8 \\
\hline & 984.8 & 828 & 396.8 \\
\hline 180 post-shutdown & 283.2 & & 376.8 \\
\hline & 296 & 361.6 & \\
\hline
\end{tabular}

Normalized Data Set

\begin{tabular}{|c|c|c|c|}
\hline $\begin{array}{c}\text { Turbulence } \\
\text { Duration } \\
\text { (minutes })\end{array}$ & $\begin{array}{c}\text { Surface Concentration } \\
\text { depth =3" } \\
(\mathrm{ppm})\end{array}$ & $\begin{array}{c}\text { Mid-level Concentration } \\
\text { depth =27" } \\
(\mathrm{ppm})\end{array}$ & $\begin{array}{c}\text { Bottom Concentraion } \\
\text { depth =63" } \\
(\mathrm{ppm})\end{array}$ \\
\hline & & & 466.4432 \\
\hline 5 & 1548.8224 & 905.2976 & 504.2976 \\
\hline & 1403.8208 & 1009.8784 & 495.3152 \\
\hline 180 & & & 459.3856 \\
\hline & 760.296 & 716.6672 & 318.2336 \\
\hline & 789.8096 & 664.056 & 302.1936 \\
\hline
\end{tabular}


9.12 APPENDIX L

NORMALIZATION METHODOLOGY FOR TOTAL OIL AND TOTAL AROMATIC HYDROCARBON CONCENTRATIONS RECORDED FROM VERTICAL TURBULENT DISPERSION REACTOR 
NORMALIZATION OF MASS LOADINGS TO VERTICAL DISPERSION REACTOR

\begin{tabular}{|c|c|c|c|c|c|}
\hline $\begin{array}{c}\text { Weathering } \\
\text { Period } \\
\text { (hours) }\end{array}$ & $\begin{array}{l}\text { Weighted } \\
\text { Volume } \\
(\mathrm{mL})\end{array}$ & $\begin{array}{c}\text { Initial } \\
\text { Weight } \\
(\mathrm{g})\end{array}$ & $\begin{array}{l}\text { Final } \\
\text { Weight } \\
(\mathrm{g})\end{array}$ & $\begin{array}{c}\text { Input Oil Weight } \\
\text { to Reactor } \\
\text { (g) }\end{array}$ & $\begin{array}{c}\text { Normalization } \\
\text { Multiplier }\end{array}$ \\
\hline 0 & 100 & & & $87.78^{\circ}$ & 1.007 \\
\hline 4 & 100 & 190 & 110 & 80 & 1.105 \\
\hline 8 & 100 & 194.8 & 114.3 & 80.5 & 1.098 \\
\hline 24 & 100 & 198.4 & 115.4 & 83 & 1.065 \\
\hline 96 & 100 & 185.8 & 96.8 & 89 & 0.893 \\
\hline 168 & 100 & & & $110.2^{* *}$ & 0.802 \\
\hline & & & & & \\
\hline & & & Average $=$ & 88.413 & \\
\hline
\end{tabular}

- determined by measured oil density alone

* entire contents of weathering flask (less water) added to reactor loading determined by mass balance 


\subsection{APPENDIX M}

TOTAL AROMATIC HYDROCARBON CONCENTRATION PROFILE, RECORDED WITHIN VERTICAL TURBULENT DISPERSION REACTOR FOR WEATHERED ARABIAN CRUDE OIL SAMPLES UNDER SIMULATED TURBULENCE 
Total Aromatics Dispersed within Water Column vs. Turbulence Duration

Weathering Period

unweathered

\begin{tabular}{|c|c|c|c|}
\hline $\begin{array}{c}\text { Turbulence } \\
\text { Duration } \\
\text { (minutes ) } \\
\end{array}$ & $\begin{array}{c}\text { Surface Concentration } \\
\text { depth = } 3^{\prime \prime} \\
(\mathrm{ppm}) \\
\end{array}$ & $\begin{array}{c}\text { Mid-level Concentration } \\
\text { depth }=27^{\prime \prime} \\
(\mathrm{ppm})\end{array}$ & $\begin{array}{c}\text { Bottom Concentraion } \\
\text { depth = 63" } \\
(\mathrm{ppm}) \\
\end{array}$ \\
\hline \multirow[t]{2}{*}{5} & 592.21 & 501.83 & 362.69 \\
\hline & 529.58 & 438.36 & 313.52 \\
\hline \multirow[t]{2}{*}{180} & 448.87 & 383.29 & 332.85 \\
\hline & 450.55 & 439.2 & 325.71 \\
\hline \multirow[t]{2}{*}{180 post-shutdown } & 12.23 & 12.02 & 2.97 \\
\hline & 16.68 & 11.59 & 3.87 \\
\hline
\end{tabular}

Normalized Data Set

\begin{tabular}{|c|c|c|c|}
\hline $\begin{array}{c}\text { Turbulence } \\
\text { Duration } \\
\text { (minutes) }\end{array}$ & $\begin{array}{c}\text { Surface Concentration } \\
\text { depth }=3^{\prime \prime} \\
(\mathrm{ppm})\end{array}$ & $\begin{array}{c}\text { Mid-level Concentration } \\
\text { depth }=27^{\prime \prime} \\
(\mathrm{ppm})\end{array}$ & $\begin{array}{c}\text { Bottom Concentraion } \\
\text { depth =63" } \\
(\mathrm{ppm})\end{array}$ \\
\hline & & & \\
\hline 5 & 596.35547 & 505.34281 & 365.22883 \\
\hline & 533.28706 & 441.42852 & 315.71464 \\
\hline & & & 335.17995 \\
\hline 180 & 452.01209 & 385.97303 & 327.98997 \\
\hline & 453.70385 & 442.2744 & 2.99079 \\
\hline 180 post-shutdown & & & 3.89709 \\
\hline & 12.31561 & 12.10414 & 11.67113 \\
\hline
\end{tabular}


Total Aromatics Dispersed within Water Column vs. Turbulence Duration

Weathering Period

4 hours

\begin{tabular}{|c|c|c|c|}
\hline $\begin{array}{c}\text { Turbulence } \\
\text { Duration } \\
\text { (minutes })\end{array}$ & $\begin{array}{c}\text { Surface Concentration } \\
\text { depth = 3" } \\
(\mathrm{ppm})\end{array}$ & $\begin{array}{c}\text { Mid-level Concentration } \\
\text { depth }=27^{\prime \prime} \\
(\mathrm{ppm})\end{array}$ & $\begin{array}{c}\text { Bottom Concentraion } \\
\text { depth =63" } \\
\text { ( ppm })\end{array}$ \\
\hline & & & 345.04 \\
\hline 5 & 690.99 & 526.22 & 305.95 \\
\hline & 596.84 & 472.41 & 308.47 \\
\hline 180 & & & 363.96 \\
\hline & 485.86 & 431.64 & 7.38 \\
\hline & 432.47 & 421.97 & 13.53 \\
\hline
\end{tabular}

Normalized Data Set

\begin{tabular}{|c|c|c|c|}
\hline $\begin{array}{c}\text { Turbulence } \\
\text { Duration } \\
\text { (minutes })\end{array}$ & $\begin{array}{c}\text { Surface Concentration } \\
\text { depth=3" } \\
(\mathrm{ppm})\end{array}$ & $\begin{array}{c}\text { Mid-level Concentration } \\
\text { depth }=27^{\prime \prime} \\
(\mathrm{ppm})\end{array}$ & $\begin{array}{c}\text { Bottom Concentraion } \\
\text { depth }=63 " \\
(\mathrm{ppm})\end{array}$ \\
\hline & & & 381.4731 \\
\hline & 763.54395 & 522.01305 & 338.07475 \\
\hline & 659.5082 & & 340.85935 \\
\hline 180 & 536.8753 & 476.9622 & 402.1758 \\
\hline & 477.87935 & 466.27685 & 8.1549 \\
\hline & & & 14.95065 \\
\hline
\end{tabular}


Total Aromatics Dispersed within Water Column vs. Turbulence Duration

\section{Weathering Period}

8 hours

\begin{tabular}{|c|c|c|c|}
\hline $\begin{array}{c}\text { Turbulence } \\
\text { Duration } \\
\text { (minutes ) } \\
\end{array}$ & $\begin{array}{c}\text { Surface Concentration } \\
\text { depth }=3^{\prime \prime} \\
(\mathrm{ppm})\end{array}$ & $\begin{array}{c}\text { Mid-level Concentration } \\
\text { depth }=27 " \\
(\mathrm{ppm})\end{array}$ & $\begin{array}{c}\text { Bottom Concentraion } \\
\text { depth = 63" } \\
(\mathrm{ppm})\end{array}$ \\
\hline \multirow[t]{2}{*}{5} & 609.87 & 605.24 & 310.15 \\
\hline & 559.84 & 640.55 & 323.6 \\
\hline \multirow[t]{2}{*}{180} & 476.19 & 458.12 & 407.68 \\
\hline & 515.28 & 474.93 & 336.21 \\
\hline \multirow[t]{2}{*}{180 post-shutdown } & 9.75 & 10.36 & 4.27 \\
\hline & 7.01 & 13.02 & 4.03 \\
\hline
\end{tabular}

Normalized Data Set

\begin{tabular}{|c|c|c|c|}
\hline $\begin{array}{l}\text { Turbulence } \\
\text { Duration } \\
\text { ( minutes ) }\end{array}$ & $\begin{array}{c}\text { Surface Concentration } \\
\text { depth }=3 " \\
(\mathrm{ppm})\end{array}$ & $\begin{array}{c}\text { Mid-level Concentration } \\
\text { depth }=27 " \\
(\mathrm{ppm})\end{array}$ & $\begin{array}{c}\text { Bottom Concentraion } \\
\text { depth = 63" } \\
(\mathrm{ppm})\end{array}$ \\
\hline \multirow[t]{2}{*}{5} & 669.63726 & 664.55352 & 340.5447 \\
\hline & 614.70432 & 703.3239 & 355.3128 \\
\hline \multirow[t]{2}{*}{180} & 522.85662 & 503.01576 & 447.63264 \\
\hline & 565.77744 & 521.47314 & 369.15858 \\
\hline \multirow[t]{2}{*}{180 post-shutdown } & 10.7055 & 11.37528 & 4.68846 \\
\hline & 7.69698 & 14.29596 & 4.42494 \\
\hline
\end{tabular}


Total Aromatics Dispersed within Water Column vs. Turbulence Duration

Weathering Period

1 day

\begin{tabular}{|c|c|c|c|}
\hline $\begin{array}{c}\text { Turbulence } \\
\text { Duration } \\
\text { (minutes })\end{array}$ & $\begin{array}{c}\text { Surface Concentration } \\
\text { depth=3" } \\
(\mathrm{ppm})\end{array}$ & $\begin{array}{c}\text { Mid-level Concentration } \\
\text { depth }=27^{\prime \prime} \\
(\mathrm{ppm})\end{array}$ & $\begin{array}{c}\text { Bottom Concentraion } \\
\text { depth =63" } \\
(\mathrm{ppm})\end{array}$ \\
\hline 5 & & & 305.11 \\
\hline & 515.29 & 456.86 & 280.31 \\
\hline & 473.67 & 486.7 & 329.91 \\
\hline 180 & & 400.53 & 294.6 \\
\hline & 497.21 & 439.62 & 5.08 \\
\hline & 465.26 & & 5.41 \\
\hline
\end{tabular}

Normalized Data Set

\begin{tabular}{|c|c|c|c|}
\hline $\begin{array}{c}\text { Turbulence } \\
\text { Duration } \\
\text { (minutes })\end{array}$ & $\begin{array}{c}\text { Surface Concentration } \\
\text { depth = 3" } \\
(\mathrm{ppm})\end{array}$ & $\begin{array}{c}\text { Mid-level Concentration } \\
\text { depth }=27^{\prime \prime} \\
(\mathrm{ppm})\end{array}$ & $\begin{array}{c}\text { Bottom Concentraion } \\
\text { depth =63" } \\
(\mathrm{ppm})\end{array}$ \\
\hline & & & \\
\hline 5 & 548.78385 & 486.5559 & 324.94215 \\
\hline & 504.45855 & 518.3355 & 298.53015 \\
\hline & & & 351.35415 \\
\hline 180 & 529.52865 & 426.56445 & 313.749 \\
\hline & 495.5019 & 468.1953 & 5.4102 \\
\hline 180 post-shutdown & 12.3966 & & 5.76165 \\
\hline & 11.9919 & 6.97575 & 5.4528 \\
\hline
\end{tabular}


Total Aromatics Dispersed within Water Column vs. Turbulence Duration

Weathering Period

4 days

\begin{tabular}{|c|c|c|c|}
\hline $\begin{array}{c}\text { Turbulence } \\
\text { Duration } \\
\text { (minutes })\end{array}$ & $\begin{array}{c}\text { Surface Concentration } \\
\text { depth = 3" } \\
(\mathrm{ppm})\end{array}$ & $\begin{array}{c}\text { Mid-level Concentration } \\
\text { depth }=27^{\prime \prime} \\
(\mathrm{ppm})\end{array}$ & $\begin{array}{c}\text { Bottom Concentraion } \\
\text { depth =63" } \\
(\mathrm{ppm})\end{array}$ \\
\hline & & & 250.46 \\
\hline 5 & 698.98 & 577.5 & 252.98 \\
\hline & 644.76 & 540.51 & 208.01 \\
\hline 180 & & & 204.22 \\
\hline & 522.85 & 430.37 & 5.82 \\
\hline & 564.47 & 493.43 & 5.54 \\
\hline
\end{tabular}

Normalized Data Set

\begin{tabular}{|c|c|c|c|}
\hline $\begin{array}{c}\text { Turbulence } \\
\text { Duration } \\
\text { (minutes) }\end{array}$ & $\begin{array}{c}\text { Surface Concentration } \\
\text { depth = 3" } \\
(\mathrm{ppm})\end{array}$ & $\begin{array}{c}\text { Mid-level Concentration } \\
\text { depth =27" } \\
(\mathrm{ppm})\end{array}$ & $\begin{array}{c}\text { Bottom Concentraion } \\
\text { depth =63" } \\
\text { ( ppm })\end{array}$ \\
\hline & & & 248.70678 \\
\hline 5 & 694.08714 & 573.4575 & 251.20914 \\
\hline & 640.24668 & 536.72643 & 206.55393 \\
\hline 180 & 519.19005 & 427.35741 & 202.79046 \\
\hline & 560.51871 & 489.97599 & 5.77926 \\
\hline & & & 5.50122 \\
\hline
\end{tabular}


Total Aromatics Dispersed within Water Column vs. Turbulence Duration

Weathering Period

7 days

\begin{tabular}{|c|c|c|c|}
\hline $\begin{array}{c}\text { Turbulence } \\
\text { Duration } \\
\text { (minutes })\end{array}$ & $\begin{array}{c}\text { Surface Concentration } \\
\text { depth =3" } \\
(\mathrm{ppm})\end{array}$ & $\begin{array}{c}\text { Mid-level Concentration } \\
\text { depth = 27" } \\
(\mathrm{ppm})\end{array}$ & $\begin{array}{c}\text { Bottom Concentraion } \\
\text { depth =63" } \\
(\mathrm{ppm})\end{array}$ \\
\hline & & & 213.89 \\
\hline 5 & 1117.46 & 577.5 & 204.22 \\
\hline & 1205.73 & 641.39 & 198.34 \\
\hline 180 & & & 186.14 \\
\hline & 466.11 & 418.6 & \\
\hline & 471.57 & 416.92 & 2.31 \\
\hline 180 post-shutdown & & & 4.32 \\
\hline & 5.46 & 4.03 & 4.74 \\
\hline
\end{tabular}

Normalized Data Set

\begin{tabular}{|c|c|c|c|}
\hline $\begin{array}{c}\text { Turbulence } \\
\text { Duration } \\
\text { (minutes })\end{array}$ & $\begin{array}{c}\text { Surface Concentration } \\
\text { depth =3" } \\
(\mathrm{ppm})\end{array}$ & $\begin{array}{c}\text { Mid-level Concentration } \\
\text { depth =27" } \\
(\mathrm{ppm})\end{array}$ & $\begin{array}{c}\text { Bottom Concentraion } \\
\text { depth }=63^{\prime \prime} \\
(\mathrm{ppm})\end{array}$ \\
\hline & & & 171.53978 \\
\hline 5 & 896.20292 & 463.155 & 163.78444 \\
\hline & 966.99546 & 514.39478 & 159.06868 \\
\hline & & & 149.28428 \\
\hline 180 & 373.82022 & 335.7172 & 1.85262 \\
\hline & 378.19914 & 334.36984 & 3.46464 \\
\hline
\end{tabular}


9.14 APPENDIX N

OBSERVED PARTICLE CHARGE DISTRIBUTIONS AND AVERAGE PARTICLE SIZE DETERMINATIONS; MEASURED BY DELSA 440 


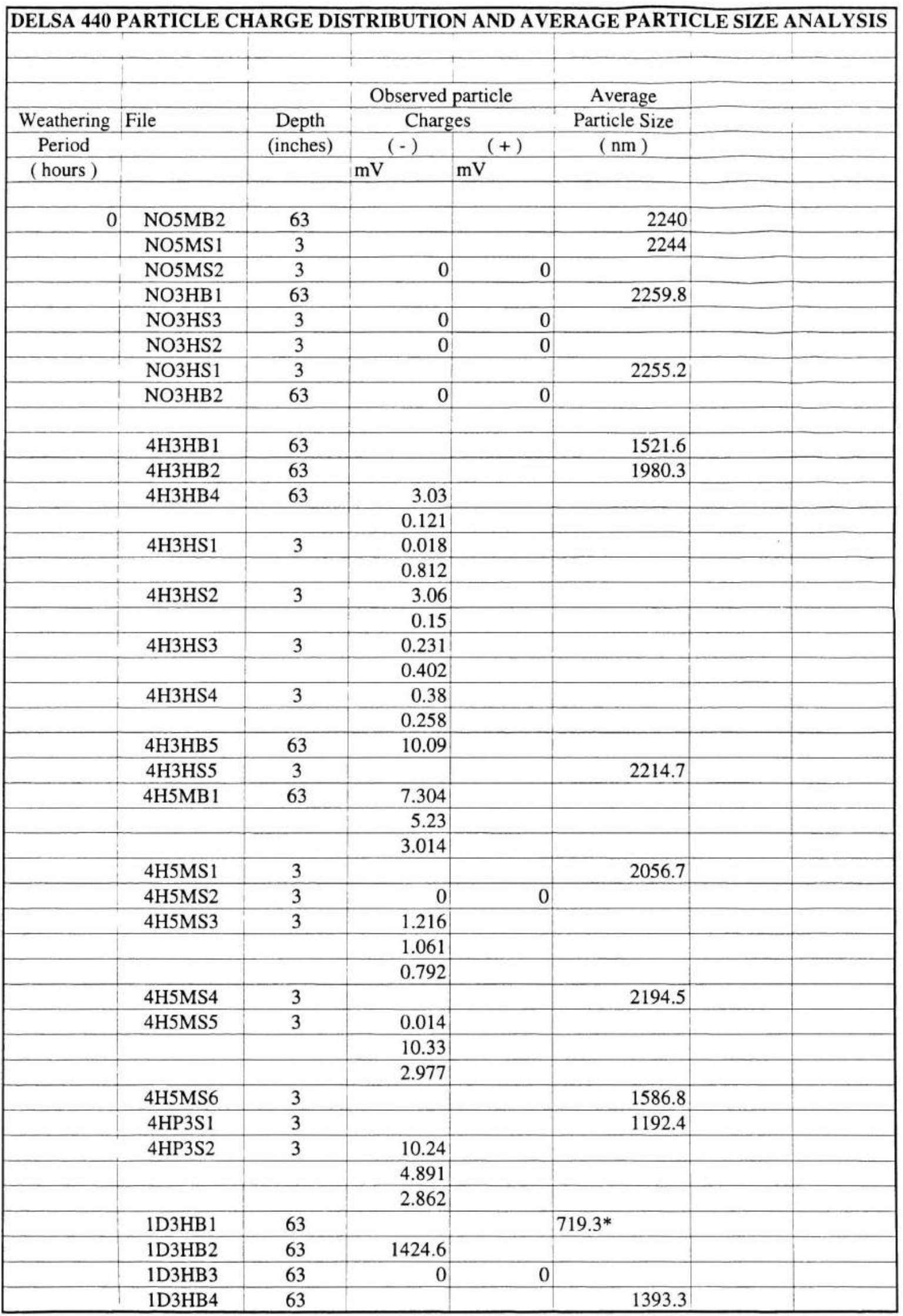




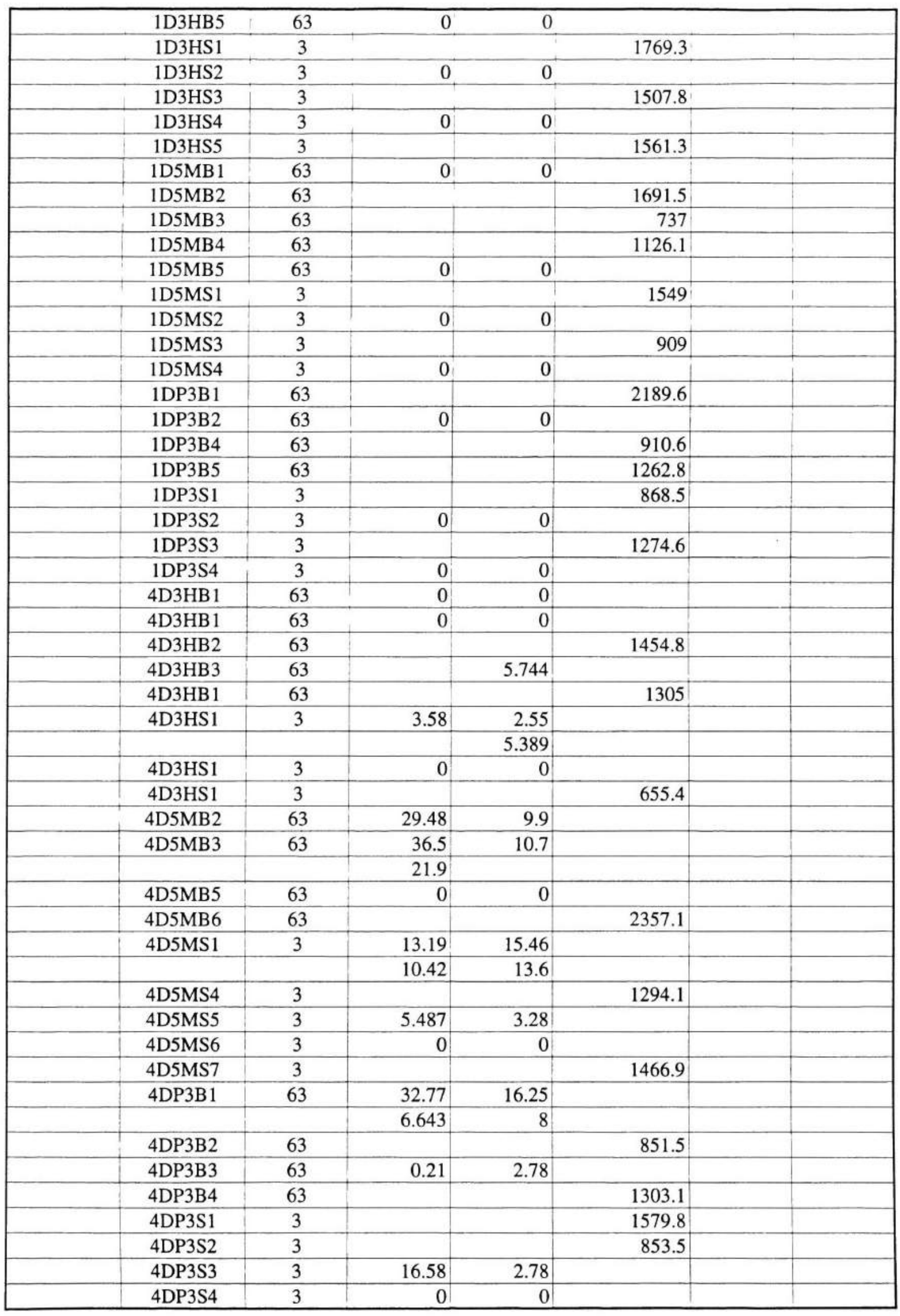




\begin{tabular}{|c|c|c|c|c|c|c|c|}
\hline & 4DP3S5 & 3 & & & 1159.9 & & \\
\hline & 7D3HB 1 & 63 & 15.198 & 3.22 & & & \\
\hline & 7D3HB3 & 63 & 10.553 & 3.057 & & & \\
\hline & & & & 5.438 & & & \\
\hline & 7D3HS1 & 3 & 10.911 & 3.426 & & & 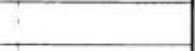 \\
\hline & & & 0.158 & & & & \\
\hline & 7D3HS2 & 3 & & & 717.4* & & \\
\hline & 7D3HS3 & 3 & 18.26 & 10.92 & & & \\
\hline & & & 15.75 & & & & \\
\hline & 7D5MB1 & 63 & 10.37 & 0.39 & & & \\
\hline & & & 7.309 & & & & \\
\hline & 7D5MB2 & 63 & & & 1463.2 & & \\
\hline & 7D5MB3 & 63 & & & $701 *$ & & \\
\hline & 7D5MB4 & 63 & 5.22 & 0.88 & & & \\
\hline & 7D5MS1 & 3 & 14.379 & 3.096 & & & : \\
\hline & & & 10.8 & & & & \\
\hline & 7D5MS3 & 3 & 0 & 0 & & & \\
\hline & 7D5MS4 & 3 & & & 2052.9 & & \\
\hline & 7DP3B2 & 63 & 0.114 & & & & \\
\hline & & & 5.194 & & & & \\
\hline & 7DP3B3 & 63 & 10.567 & & & & \\
\hline & 7DP3B4 & 63 & & & $650^{*}$ & . & \\
\hline & 7DP3S1 & 3 & 7.457 & & & & \\
\hline & 7DP3S2 & 3 & & & $695 *$ & & \\
\hline & $8 \mathrm{H} 3 \mathrm{HB} 1$ & 63 & & & 1933.4 & & \\
\hline & $8 \mathrm{H} 3 \mathrm{HB} 2$ & 63 & 10.9 & & & & \\
\hline & & & 7.68 & & & & \\
\hline & $8 \mathrm{H} 3 \mathrm{HB} 3$ & 63 & & & $498^{*}$ & & \\
\hline & $8 \mathrm{H} 3 \mathrm{HB} 4$ & 63 & 21.73 & & & & \\
\hline & & & 10.75 & & & & \\
\hline & $8 \mathrm{H} 3 \mathrm{HS} 1$ & 3 & & & 2069.6 & & \\
\hline & $8 \mathrm{H} 3 \mathrm{HS} 2$ & 3 & 0 & 0 & & & \\
\hline & $8 \mathrm{H} 3 \mathrm{HS} 3$ & 3 & 11.19 & 2.89 & & & \\
\hline & $8 \mathrm{H} 3 \mathrm{HS} 4$ & 3 & & & 837.7 & & \\
\hline & $8 \mathrm{H} 5 \mathrm{MB} 1$ & 63 & & & 986.5 & & \\
\hline & $8 \mathrm{H} 5 \mathrm{MB} 3$ & 63 & 16.484 & 4.472 & & & \\
\hline & $8 \mathrm{H} 5 \mathrm{MB} 4$ & 63 & 11.273 & 0.15 & & & \\
\hline & $8 \mathrm{H} 5 \mathrm{MS} 1$ & 3 & & & 2063 & & \\
\hline & $8 \mathrm{H} 5 \mathrm{MS} 1 \mathrm{~A}$ & 3 & & & 2050.3 & & \\
\hline & $8 \mathrm{H} 5 \mathrm{MS} 2$ & 3 & 13.928 & 5.346 & & & \\
\hline & $8 \mathrm{H} 5 \mathrm{MS} 2 \mathrm{~A}$ & 3 & 11.288 & 2.893 & & & \\
\hline & $8 \mathrm{H} 5 \mathrm{MS} 3$ & 3 & 28.247 & 11.11 & & & \\
\hline & 8HP3B1 & 63 & & & 2183 & & \\
\hline & 8HP3B1A & 63 & 0 & 0 & & & \\
\hline & 8HP3B2 & 63 & & & 503 & & \\
\hline & 8HP3B3 & 63 & & & 2114.5 & & \\
\hline & 8HP3B4 & 63 & 3.219 & & & & \\
\hline & & & 5.551 & & & & \\
\hline & & & 0.11 & & & & \\
\hline & $8 \mathrm{HP} 3 \mathrm{~S} 1$ & 3 & & & 1007 & & \\
\hline & $8 \mathrm{HP} 3 \mathrm{~S} 2$ & 3 & & & 978 & & \\
\hline & $8 \mathrm{HP} 3 \mathrm{~S} 3$ & 3 & 11.245 & & & & \\
\hline
\end{tabular}


Page 4 


\subsection{APPENDIX O}

RELATIVE TOXICITY DATA EXPRESSED AS EC-50 VALUES FOR WEATHERED OIL SAMPLES 


\begin{tabular}{|c|c|c|c|c|}
\hline \multicolumn{2}{|c|}{ MICROTOX ANALYSIS } & & \multirow[b]{2}{*}{ NORMALIZATION } & \multirow[b]{2}{*}{ ADJUSTED } \\
\hline SAMPLE I.D & AROMATIC HYDROCARBON & EC-50 VALUE & & \\
\hline & CONCENTRATION (PPM) & (PPM) & MULTIPLIER & EC-50 VALUE (PPM) \\
\hline & [NORMALIZED DATA] & & & \\
\hline & & & & \\
\hline NO3HMTB1 & 335.18 & 0.2422 & 1.007 & 0.2438854 \\
\hline NO3HMTB2 & 327.99 & 0.1638 & 1.007 & 0.1649466 \\
\hline & & & & \\
\hline 4H3HMTB1 & 340.86 & 0.2077 & 1.105 & 0.2285085 \\
\hline 4H3HMTB2 & 402.18 & 0.3283 & 1.105 & 0.3627715 \\
\hline & & & & \\
\hline 8H3HMTB1 & 447.63 & 0.4529 & 1.098 & 0.4972842 \\
\hline $8 \mathrm{H} 3 \mathrm{HMTB2}$ & 368.16 & 0.3293 & 1.098 & 0.3615714 \\
\hline & & & & \\
\hline 1D3HMTB1 & 351.35 & 0.2862 & 1.065 & 0.304803 \\
\hline 1D3HMTB2 & 313.75 & 0.2732 & 1.065 & 0.290958 \\
\hline 4D3HMTB1 & 20655 & 02501 & 0003 & 02482402 \\
\hline 4D3HMTB2 & 202.78 & 0.1894 & 0.993 & 0.1880742 \\
\hline & & & & \\
\hline 7D3HMTB1 & 159.07 & 0.1349 & 0.802 & 0.1081898 \\
\hline 7D3HMTB2 & 149.28 & 0.0549 & 0.802 & 0.0440298 \\
\hline & & & & \\
\hline
\end{tabular}

